\title{
Advanced Two-Dimensional Heterojunction Photocatalysts of Stoichiometric and Non-Stoichiometric Bismuth Oxyhalides with Graphitic Carbon Nitride for Sustainable Energy and Environmental Applications
}

\author{
Kishore Sridharan ${ }^{1, * \mathbb{D}}$, Sulakshana Shenoy ${ }^{2} \mathbb{D}$, S. Girish Kumar $^{3} \mathbb{D}$, Chiaki Terashima ${ }^{4}(\mathbb{D}$, Akira Fujishima 4 \\ and Sudhagar Pitchaimuthu 5,*(D)
}

Citation: Sridharan, K.; Shenoy, S.; Kumar, S.G.; Terashima, C.; Fujishima, A.; Pitchaimuthu, S. Advanced Two-Dimensional Heterojunction Photocatalysts of Stoichiometric and Non-Stoichiometric Bismuth Oxyhalides with Graphitic Carbon Nitride for Sustainable Energy and Environmental Applications. Catalysts 2021, 11, 426. https:// doi.org/10.3390/catal11040426

Academic Editor: Juan Matos Lale

Received: 9 March 2021

Accepted: 23 March 2021

Published: 26 March 2021

Publisher's Note: MDPI stays neutral with regard to jurisdictional claims in published maps and institutional affiliations.

Copyright: (c) 2021 by the authors Licensee MDPI, Basel, Switzerland. This article is an open access article distributed under the terms and conditions of the Creative Commons Attribution (CC BY) license (https:// creativecommons.org/licenses/by/ $4.0 /)$
1 Department of Nanoscience and Technology, University of Calicut, Thenhipalam 673635, India

2 Department of Physics, National Institute of Technology Karnataka, Mangalore 575025, India; shenoy26sulakshana@gmail.com

3 Department of Chemistry, School of Engineering \& Technology, CMR University, Bangalore 562149, India; girichem@yahoo.co.in

4 Photocatalysis International Research Center, Research Institute for Science and Technology, Tokyo University of Science, 2641 Yamazaki, Noda Chiba 278-8510, Japan; terashima@rs.tus.ac.jp (C.T.); fujishima_akira@rs.tus.ac.jp (A.F.)

5 Multifunctional Photocatalyst and Coatings Group, SPECIFIC, Materials Research Centre, Faculty of Science and Engineering, Swansea University, Swansea SA1 8EN, UK

* Correspondence: sridharankishore@uoc.ac.in or sridharankishore@gmail.com (K.S.); s.pitchaimuthu@swansea.ac.uk or vedichi@gmail.com (S.P.)

Abstract: Semiconductor-based photocatalysis has been identified as an encouraging approach for solving the two main challenging problems, viz., remedying our polluted environment and the generation of sustainable chemical energy. Stoichiometric and non-stoichiometric bismuth oxyhalides ( $\mathrm{BiOX}$ and $\mathrm{Bi}_{\mathrm{x}} \mathrm{O}_{\mathrm{y}} \mathrm{X}_{\mathrm{z}}$ where $\mathrm{X}=\mathrm{Cl}, \mathrm{Br}$, and $\mathrm{I}$ ) are a relatively new class of semiconductors that have attracted considerable interest for photocatalysis applications due to attributes, viz., high stability, suitable band structure, modifiable energy bandgap and two-dimensional layered structure capable of generating an internal electric field. Recently, the construction of heterojunction photocatalysts, especially $2 \mathrm{D} / 2 \mathrm{D}$ systems, has convincingly drawn momentous attention practicably owing to the productive influence of having two dissimilar layered semiconductors in face-to-face contact with each other. This review has systematically summarized the recent progress on the $2 \mathrm{D} / 2 \mathrm{D}$ heterojunction constructed between $\mathrm{BiOX} / \mathrm{Bi}_{\mathrm{x}} \mathrm{O}_{\mathrm{y}} \mathrm{X}_{\mathrm{z}}$ with graphitic carbon nitride $\left(\mathrm{g}-\mathrm{C}_{3} \mathrm{~N}_{4}\right)$. The band structure of individual components, various fabrication methods, different strategies developed for improving the photocatalytic performance and their applications in the degradation of various organic contaminants, hydrogen $\left(\mathrm{H}_{2}\right)$ evolution, carbon dioxide $\left(\mathrm{CO}_{2}\right)$ reduction, nitrogen $\left(\mathrm{N}_{2}\right)$ fixation and the organic synthesis of clean chemicals are summarized. The perspectives and plausible opportunities for developing high performance $\mathrm{BiOX} / \mathrm{Bi}_{\mathrm{x}} \mathrm{O}_{\mathrm{y}} \mathrm{X}_{\mathrm{z}}-\mathrm{g}-\mathrm{C}_{3} \mathrm{~N}_{4}$ heterojunction photocatalysts are also discussed.

Keywords: 2D materials; photocatalysis; heterojunction; bismuth oxyhalides; graphitic carbon nitride

\section{Introduction}

Excessive demand for pharmaceutical, personal care, agricultural and industrial products driven by the continued growth of the world population has inevitably escalated the discharge of organic contaminants into the environment [1]. The steadily increasing concentration of organic contaminants primarily originating from pharmaceutical and personal care products in municipal wastewaters of many urban cities globally is making microorganisms resistant to drugs [2]. Undoubtedly, these organic contaminants pose a huge threat to the environment and human health as they have demonstrated severe ecological risk 
for mutagenesis, teratogenesis and carcinogenicity [3]. Therefore, in addition to finding sustainable solutions to our global energy crisis and eliminating the steadily increasing $\mathrm{CO}_{2}$ concentration from the environment, the removal of these organic contaminants with high chemical stability is another highly challenging task [4]. Several methods based on chemical [5] and biological [6] techniques and advanced oxidation processes [7] have been employed for the complete removal of organic contaminants from wastewater. However, almost all strategies failed to achieve complete degradation, and the search for a green, efficient and economically viable technology continued. In 1972, pioneering work reported by Fujishima and Honda revealed that UV light irradiated on the surface of a $\mathrm{TiO}_{2}$ electrode generated free radicals for the decomposition of water into hydrogen and oxygen. Later on, it was revealed that the photogenerated free radicals emanating from semiconductors under UV/Visible light excitation could also cleavage the chemical bonds in the molecular organic contaminants adsorbed on their surfaces [8]. In this regard, heterogeneous semiconductor photocatalysis - categorized as another form of advanced oxidation process-has received an overwhelming research interest as a "one-step solution" for addressing the energy and environmental issues, viz., the generation of hydrogen gas through light-water splitting reaction, the reduction of $\mathrm{CO}_{2}$ into hydrocarbons and to completely break down organic contaminants through redox reactions involving the radical species [9]. Despite nanostructured $\mathrm{TiO}_{2}$ being a robust and chemically stable semiconductor, its wide bandgap energy $(3.2 \mathrm{eV})$ demands UV light for its excitation. Since the visible light is predominant in the solar spectrum and with UV light being insignificant (just $\sim 4 \%$ ), researchers swiftly moved to utilize nanostructured semiconductors with a narrower bandgap energy (such as $\mathrm{CdS}, \mathrm{Fe}_{2} \mathrm{O}_{3}, \mathrm{WO}_{3}$, etc.) for efficiently utilizing the inexhaustible sunlight energy [10,11].

Since the discovery of graphene, semiconductors with 2D layered structures have greatly influenced the researchers to study them for applications in photocatalysis due to their unique sheet-like morphology with one-dimensionally confined electrons producing exceptional physio-chemical, optical and electronic properties [12]. In addition to the ease of fabrication, other interesting features of 2D semiconductors exclusively for photocatalytic applications are their large specific surface area with many photoactive sites and customizable thickness leading to easy adjustments to the bandgap energy and light absorption efficiency. Further, the atomically thin 2D layered morphology enables strong in-plane bond formation, facilitating easy heterostructure construction (on substrates or with other 2D semiconductors through weak van der Waals interaction) and enhancing the rate of the photocatalytic reactions due to the shortened transport path [13].

Among the various 2D semiconductors for photocatalysis applications, bismuth oxyhalides (referred to hereafter as $\mathrm{BiOX}$, where $\mathrm{X}=\mathrm{Cl}, \mathrm{Br}$ and I) - a group of V-VI-VII ternary compounds with stoichiometric form-have become the prime choice for researchers owing to their nontoxicity, layered morphology, unique crystal structure, suitable band structure, variable bandgap energy and excellent chemical stability ensuring corrosion resistance in the solution medium for long term operations [14]. The stoichiometric BiOX possessing tetragonal matlockite polymorph (PbFCl-type; space group - $\mathrm{P} 4 / \mathrm{nmm}$ ) crystallize into layered structures consisting of patterned [X-Bi-O-Bi-X] slices stacked together by the nonbonding van der Waals interaction through the halogen atoms along the c-axis, as depicted in Figure 1. In each [X-Bi-O-Bi-X] layer, the central Bi atom is surrounded by four oxygen and four halogen atoms, generating an asymmetric decahedral geometry [15]. The open crystalline structure, indirect bandgap, strong covalent bonding combined with weak interlayer van der Waals interaction, and excellent electrical, optical and mechanical properties are the features that endow $\mathrm{BiOX}$ as a promising candidate for light induced redox reactions [16]. However, poor light absorption, restricted utilization and limited chemical stability are some of the shortcomings of BiOX. 


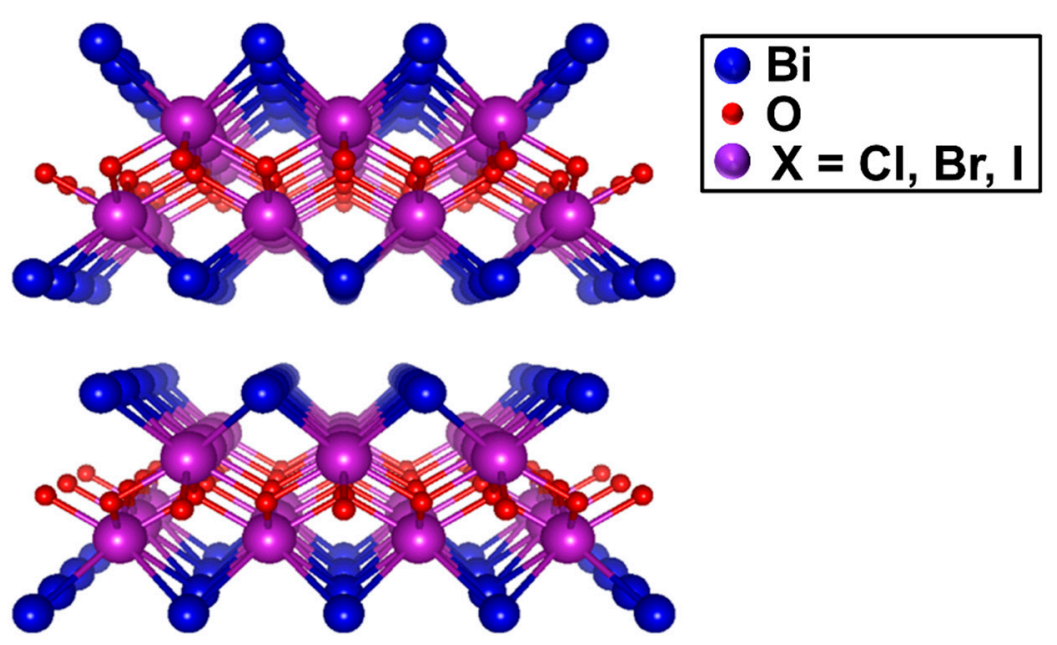

Figure 1. Crystal structure of the BiOX systems (space group $P 4 / n m m, \mathrm{D}_{4 \mathrm{~h}}$ symmetry) with stoichiometric X-Bi-O-Bi-X Bi-layers stacked along the $\mathrm{c}$ axis. Bismuth, oxygen, and halide ions are denoted by purple, red and blue spheres, respectively.

On the other hand, bismuth rich-bismuth oxyhalides (referred to hereafter as $\mathrm{Bi}_{\mathrm{x}} \mathrm{O}_{\mathrm{y}} \mathrm{X}_{\mathrm{z}}$ ) with non-stoichiometric form also have a layered structure similar to $\mathrm{BiOX}$, with strong covalent bonding and weak interlayer van der Walls interactions. Generally, the band structure of a semiconductor is governed by its chemical components to a great extent. In non-stoichiometric $\mathrm{Bi}_{\mathrm{x}} \mathrm{O}_{\mathrm{y}} \mathrm{X}_{\mathrm{z}}$, the replacement of the halogen atoms in its lattice correspondingly led to modified band structure and subsequently the optical absorption edge and band redox potentials [17]. Most importantly, the negative conduction band positions of $\mathrm{Bi}_{\mathrm{x}} \mathrm{O}_{\mathrm{y}} \mathrm{X}_{\mathrm{z}}$ facilitate its widespread utilization for photocatalytic applications [18].

Graphitic carbon nitride $\left(\mathrm{g}-\mathrm{C}_{3} \mathrm{~N}_{4}\right)$ is another exquisite 2D semiconductor that has been flourishing in the recent years for applications in photocatalysis due to its tri-striazine ring structure, appealing electronic band structure, medium bandgap $(2.7 \mathrm{eV})$, and excellent chemical and thermal stability [19]. In addition, the earth-abundant carbon and nitrogen elements in $\mathrm{g}-\mathrm{C}_{3} \mathrm{~N}_{4}$ can be easily prepared via one-step polymerization of abundantly available inexpensive nitrogen-rich precursors, such as urea, thiourea, melamine, cyanamide and dicyandiamide [20,21]. Nevertheless, pristine g- $\mathrm{C}_{3} \mathrm{~N}_{4}$ also suffers from shortcomings such as high excitation energy, low charge carrier mobility, the rapid recombination of photogenerated charge carriers, and narrow visible light absorption efficiency [22].

Thus, integrating $\mathrm{BiOX} / \mathrm{Bi}_{\mathrm{x}} \mathrm{O}_{\mathrm{y}} \mathrm{X}_{\mathrm{z}}$ with $\mathrm{g}-\mathrm{C}_{3} \mathrm{~N}_{4}$ would be an ideal strategy to overcome many of the demerits associated with individual components. The 2D layered structures of both $\mathrm{BiOX} / \mathrm{Bi}_{\mathrm{x}} \mathrm{O}_{\mathrm{y}} \mathrm{X}_{\mathrm{z}}$ and $\mathrm{g}-\mathrm{C}_{3} \mathrm{~N}_{4}$ conveniently promote the construction of a heterojunction and, furthermore, the favourable band energy between them can facilitate enhanced photocatalytic performance [23-29]. Several review articles on single component 2D semiconductor photocatalysts concentrating primarily on $\mathrm{BiOX}, \mathrm{Bi}_{\mathrm{x}} \mathrm{O}_{\mathrm{y}} \mathrm{X}_{\mathrm{z}}$ and $\mathrm{g}-\mathrm{C}_{3} \mathrm{~N}_{4}$ have been published [15,30-58]. Nonetheless, a review article accounting the progress of heterojunction photocatalysts based on $\mathrm{BiOX}$ and $\mathrm{Bi}_{\mathrm{x}} \mathrm{O}_{\mathrm{y}} \mathrm{X}_{\mathrm{z}}$ with $\mathrm{g}-\mathrm{C}_{3} \mathrm{~N}_{4}$ is rarely reported. Since there is a consistent upsurge in the research trend on $\mathrm{BiOX}$ based photocatalysts as evidenced from the literature survey presented in Figure 2, a review article is needed to fill the gaps and to account the recent progress. Therefore, in this review, we have presented a summary on the band structure of $\mathrm{Bi}_{\mathrm{x}} \mathrm{O}_{\mathrm{y}} \mathrm{X}_{\mathrm{z}}$ and have furnished information on the various methods of coupling $\mathrm{BiOX} / \mathrm{Bi}_{\mathrm{x}} \mathrm{O}_{\mathrm{y}} \mathrm{X}$ and $\mathrm{g}-\mathrm{C}_{3} \mathrm{~N}_{4}$ to fabricate heterojunction photocatalysts for organic contaminant degradation, $\mathrm{H}_{2}$ generation, $\mathrm{CO}_{2}$ reduction, $\mathrm{N}_{2}$ fixation and organic synthesis applications. Further, the various strategies for improving the performance of g- $\mathrm{C}_{3} \mathrm{~N}_{4}-\mathrm{BiOX} / \mathrm{Bi}_{\mathrm{x}} \mathrm{O}_{\mathrm{y}} \mathrm{X}_{\mathrm{z}}$ heterojunction photocatalysts, viz., the creation of defects, the role of facets, integration with other semiconductors, metals and carbon materials are discussed. 
Additionally, the future prospects of $\mathrm{BiOX} / \mathrm{Bi}_{\mathrm{x}} \mathrm{O}_{\mathrm{y}} \mathrm{X}_{\mathrm{z}}-\mathrm{g}-\mathrm{C}_{3} \mathrm{~N}_{4}$ heterojunction photocatalysts for broader energy and environmental applications are deliberated.
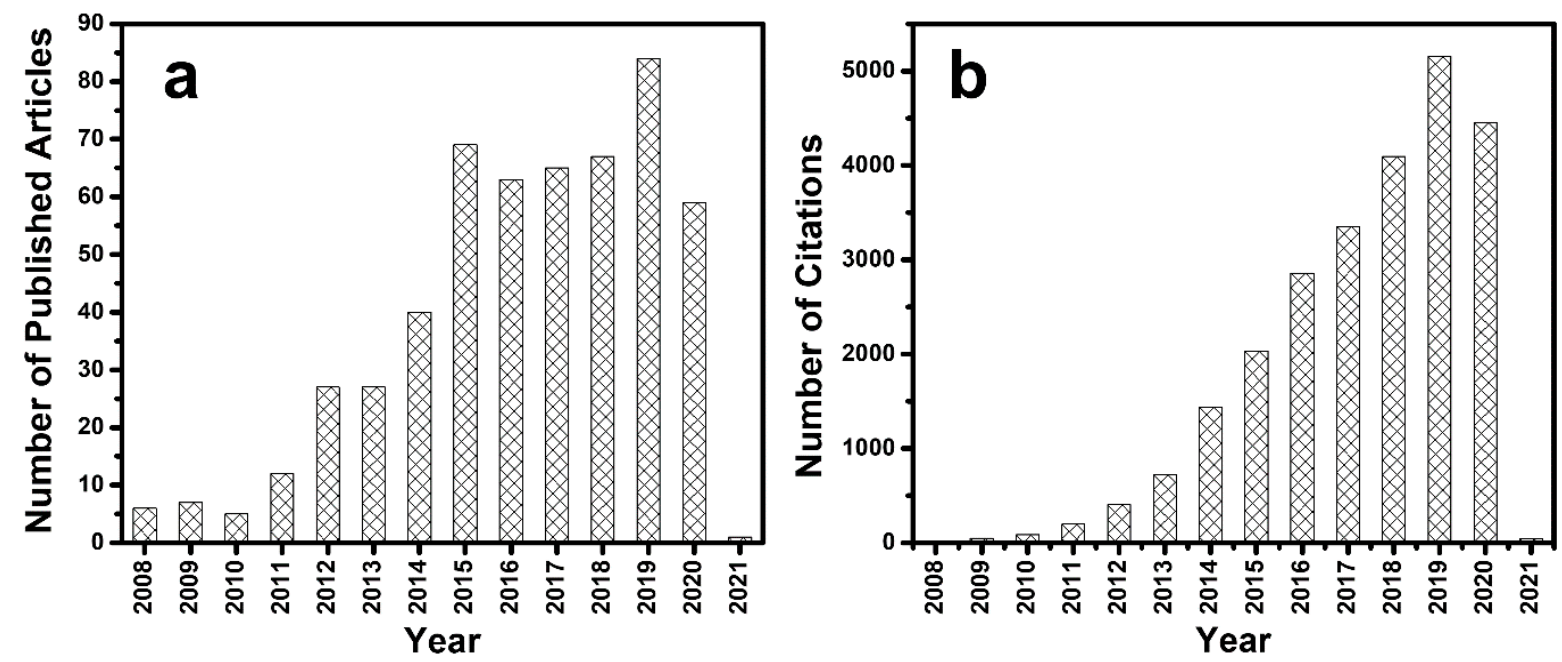

Figure 2. (a) Number of articles published and (b) number of citations since 2008 with topic keywords "BiOX" and "photocatal*" adapted from the Web of Science, dated 17 November 2020.

\section{Electronic Band Structure of BiOX, $B i_{x} O_{y} X_{z}$ and $g-C_{3} N_{4}$}

The band structure of the material is the crucial parameter that dictates the light absorption capacity, charge carrier dynamics and generation of free radicals. In the case of $\mathrm{BiOX}, \mathrm{O} 2 \mathrm{p}$ and $\mathrm{X} n \mathrm{p}$ states ( $n=3,4$ and 5 for $\mathrm{X}=\mathrm{Cl}, \mathrm{Br}$, and I, respectively) constitute the valance band minimum (VBM), while the conduction band maximum (CBM) is derived from Bi $6 s$ and the Bi $6 p$ states. The largely dispersed Bi $6 s$ orbital facilitates the mobility of photoinduced holes in the VB (valence band) and is beneficial for the oxidation reaction. The energy bandgap values and the redox potentials of $\mathrm{BiOX}$ are vastly related to the atomic numbers of $X$ and the composition of the layered structure [59]. Therefore, the optical absorption in $\mathrm{BiOX}$ can be tailored via varying the halogen species or $\mathrm{Bi} / \mathrm{X}$ ratios. Increasing the atomic number of $X$ leads to a change in the colour and bandgap energy of BiOX from white $(\mathrm{BiOCl}, 3.2 \mathrm{eV})$ to yellow $(\mathrm{BiOBr}, 2.7 \mathrm{eV})$ and red $(\mathrm{BiOI}, 1.7 \mathrm{eV})$, thus maximizing their light absorption capacities [60]. The open crystalline structure has a layered SillenAurivillius related oxide structure composed of $\left[\mathrm{Bi}_{2} \mathrm{O}_{2}\right]$ layers sandwiched between two slabs of $[\mathrm{X}]$ ions, and the electrostatic potential difference between the slabs generates a static internal electric field (IEF). The static IEF in BiOX can effectively split and transit the photogenerated electrons and holes [61-63]. However, BiOX as a photocatalyst could be employed for the degradation of organic pollutants alone as its positive $\mathrm{CB}$ (conduction band) potential restricts it from being used for other photocatalytic applications such as $\mathrm{H}_{2}$ generation, $\mathrm{CO}_{2}$ reduction, $\mathrm{N}_{2}$ fixation and organic synthesis.

On the other hand, non-stoichiometric $\mathrm{Bi}_{\mathrm{x}} \mathrm{O}_{\mathrm{y}} \mathrm{X}_{\mathrm{z}}$ with increased $\mathrm{Bi}$ content are reported to promote the reduction power of photogenerated electrons and increase the thermodynamic force for initiating many reduction reactions that were impossible to be carried out using $\mathrm{BiOX}[64,65]$. For instance, compared with $\mathrm{BiOX}$, the changes in the $\mathrm{Bi}, \mathrm{O}$, and $\mathrm{X}$ proportions result in the variation of orbital hybridization and uplifting of the bottom of the $\mathrm{CB}$, leading to the water splitting for $\mathrm{H}_{2}$ generation as was reported in $\mathrm{Bi}_{4} \mathrm{O}_{5} \times 2$ $(\mathrm{X}=\mathrm{Br}$ and $\mathrm{I})[66,67]$. In addition to $\mathrm{H}_{2}$ generation, the increased $\mathrm{CB}$ also promoted photocatalytic molecular oxygen activation in $\mathrm{Bi}_{24} \mathrm{O}_{31} \mathrm{Cl}_{10}$. Further, the $\mathrm{Bi}$-rich $\mathrm{Bi}_{\mathrm{x}} \mathrm{O}_{\mathrm{y}} \mathrm{X}_{\mathrm{z}}$ possesses enhanced light-harvesting ability that is attributed to the modulated band structure, thus breaking the bottleneck of limited photoabsorption caused from the wide bandgap energy of $\mathrm{BiOCl}$ and $\mathrm{BiOBr}$ [68]. The higher photon absorption efficiency of $\mathrm{Bi}_{\mathrm{x}} \mathrm{O}_{\mathrm{y}} \mathrm{X}_{\mathrm{z}}$ in comparison to $\mathrm{BiOX}$ induces greater electric field intensity, which in turn leads to large dipole moment. The larger dipole moment and wider interlayer spacing in $\mathrm{Bi}_{\mathrm{x}} \mathrm{O}_{\mathrm{y}} \mathrm{X}_{\mathrm{z}}$ boosted by the large 
polarization force and polarization space lead to increased IEF, which in turn enhances the separation efficiency of the photogenerated charge carriers.

Electronic band structure, redox levels of the $\mathrm{CB}$ and VB and the bandgap energy of g- $\mathrm{C}_{3} \mathrm{~N}_{4}$ were studied both theoretically and experimentally. Theoretical calculations estimated the bandgap energy of the melem molecule, polymeric melon and fully condensed g- $\mathrm{C}_{3} \mathrm{~N}_{4}$ to be 3.5, 2.6 and $2.1 \mathrm{eV}$, respectively [69-71]. The bandgap energy value of $2.6 \mathrm{eV}$ calculated for polymeric melon was consistent with the experimentally measured value of $2.7 \mathrm{eV}$ for defect containing bulk g- $\mathrm{C}_{3} \mathrm{~N}_{4}$ [70]. The CBM and VBM positions for $\mathrm{g}_{-} \mathrm{C}_{3} \mathrm{~N}_{4}$ estimated through the density functional theory were -1.12 and $+1.57 \mathrm{eV}$, respectively. Interestingly, the experimental investigations through the valence band $\mathrm{X}$-ray photoelectron spectroscopy confirmed the $\mathrm{VBM}$ position of $\mathrm{g}-\mathrm{C}_{3} \mathrm{~N}_{4}$ at $+1.53 \mathrm{eV}$, which was almost consistent with the theoretical calculations [72]. Therefore, the position of the CBM $(-1.12 \mathrm{eV})$ is predicted to be satisfactory for $\mathrm{H}_{2}$ generation, while that of the VBM provides a thermodynamic driving force for $\mathrm{O}_{2}$ evolution reaction. Wavefunction studies revealed that the $\mathrm{VB}$ and $\mathrm{CB}$ of $\mathrm{g}-\mathrm{C}_{3} \mathrm{~N}_{4}$ serving as independent sites for the oxidation and reduction reactions during water splitting are mainly driven by the nitrogen $\mathrm{P}_{\mathrm{Z}}$ orbitals and carbon $\mathrm{P}_{\mathrm{z}}$ orbitals, respectively. Further, the redox potential levels of water calculated by ab initio thermodynamics indicated that both the reduction and oxidation level of water splitting are located within the bandgap of $\mathrm{g}-\mathrm{C}_{3} \mathrm{~N}_{4}$ [69]. Another theory using the many-body Green's function reported that lone pair electrons of nitrogen atoms are mainly responsible for the formation of the VB and electronic structure [20,73]. Additionally, it was proposed that the $N 2 p$ orbital overlapping the $C 2 p$ orbital mainly contributes to the VB and $C B$ of $g-C_{3} N_{4}$, respectively [74]. As observed from Figure 3, the CB position of $g-C_{3} N_{4}$ and many of the $\mathrm{Bi}_{\mathrm{x}} \mathrm{O}_{\mathrm{y}} \mathrm{X}_{\mathrm{z}}$ are suitable for photocatalytic $\mathrm{H}_{2}$ generation, $\mathrm{CO}_{2}$ reduction, $\mathrm{N}_{2}$ fixation and molecular oxygen activation in addition to their potential to be utilized in the degradation of organic pollutants. Further, it is evident from Figure 3 that the VB and CB levels of g- $\mathrm{C}_{3} \mathrm{~N}_{4}$ match well with those of $\mathrm{BiOX}$ and $\mathrm{Bi}_{\mathrm{x}} \mathrm{O}_{\mathrm{y}} \mathrm{X}_{\mathrm{z}}$ for the fabrication of efficient $2 \mathrm{D} / 2 \mathrm{D}$ heterojunction photocatalysts.

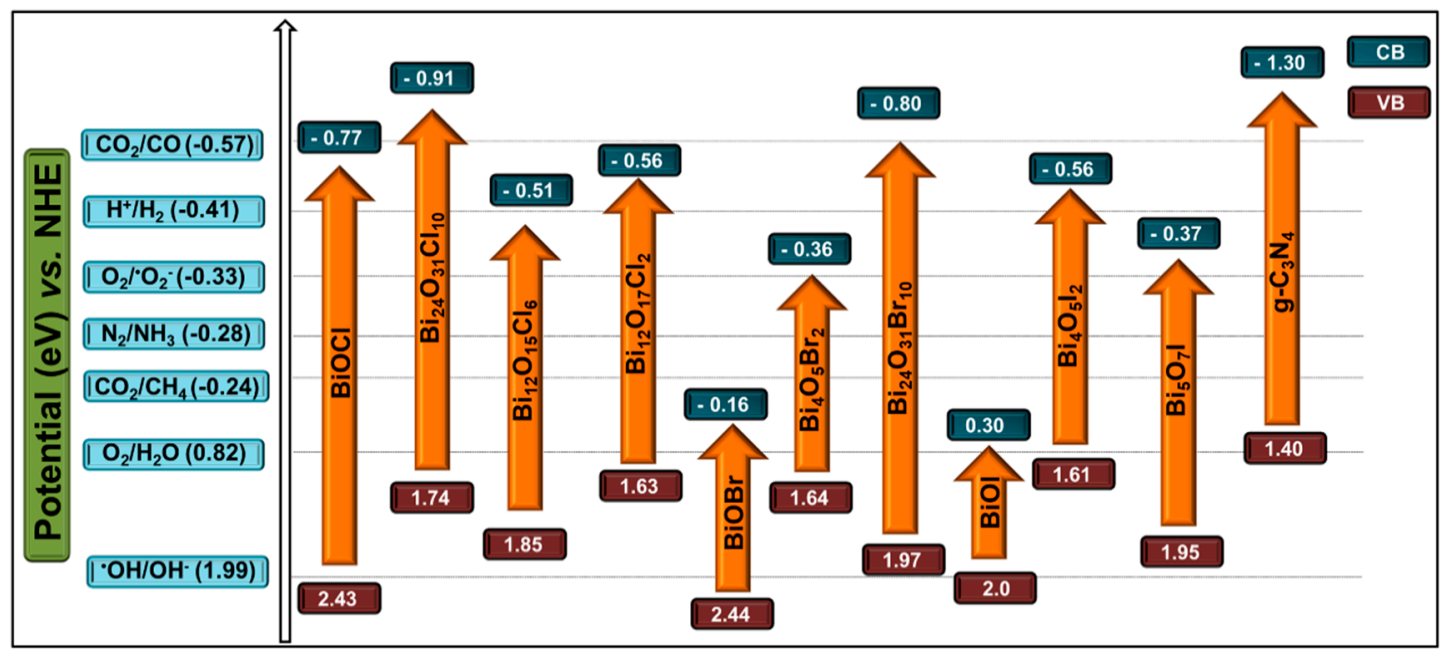

Figure 3. Conduction and valence band (CB and VB) positions of $g-\mathrm{C}_{3} \mathrm{~N}_{4}, \mathrm{BiOX}$, and some of the $\mathrm{Bi}_{\mathrm{x}} \mathrm{O}_{\mathrm{y}} \mathrm{X}_{\mathrm{z}}$ photocatalysts vs. Normal Hydrogen Electrode $(\mathrm{NHE})$ at $\mathrm{pH}=7$. The redox potentials of different chemical reactions are compared in this figure.

\section{Fabrication of $\mathrm{BiOX} / \mathrm{Bi}_{\mathrm{x}} \mathrm{O}_{\mathrm{y}} \mathrm{X}_{\mathrm{z}}-\mathrm{g}-\mathrm{C}_{3} \mathrm{~N}_{4}$ Heterojunction Photocatalysts}

Fabrication is a significant step involved in tailoring the band structure of photocatalysts due to its dependence on the chemical composition. Morphology, shape, size and surface area are some of the critical parameters that play a determinant role in the adsorption properties and photocatalytic activity. Benefiting from the large specific surface area, $2 \mathrm{D}$ semiconductors can provide abundant surface active sites. More importantly, the greatly reduced thickness of $2 \mathrm{D}$ semiconductors relative to bulk counterparts shortens the 
bulk carrier diffusion distance and improves the charge separation. Further, the surface charge separation efficiency is enhanced by the creation of surface defects such as oxygen vacancies during the fabrication of the 2D semiconductors.

The typical bismuth metal precursors utilized for synthesizing $\mathrm{BiOX}$ and $\mathrm{Bi}_{\mathrm{x}} \mathrm{O}_{\mathrm{y}} \mathrm{X}_{\mathrm{z}}$ are $\mathrm{Bi}\left(\mathrm{NO}_{3}\right)_{3} \cdot 5 \mathrm{H}_{2} \mathrm{O}, \mathrm{NaBiO}_{3} \cdot 2 \mathrm{H}_{2} \mathrm{O}, \mathrm{Bi}_{2} \mathrm{O}_{3}, \mathrm{BiCl}_{3}$ and $\mathrm{BiI}_{3}$, while the halogen precursors include $\mathrm{KX}, \mathrm{NaX}, \mathrm{HX}, \mathrm{CTAX}(\mathrm{X}=\mathrm{Cl}, \mathrm{Br}$ or I) and ionic liquids containing halogen elements. Various solution based fabrication techniques such as the electrostatic self-assembly approach, the hydrothermal method, the ionic liquid-assisted method, the impregnation method, the solid-phase calcination step, the solvothermal method, precipitation, the reflux process and the ultrasound-assisted water bath technique are used in the synthesis of $\mathrm{BiOX}$ and $\mathrm{Bi}_{\mathrm{x}} \mathrm{O}_{\mathrm{y}} \mathrm{X}_{\mathrm{z}}$. On the other hand, the precursors used for synthesizing g- $\mathrm{C}_{3} \mathrm{~N}_{4}$ through the most typical thermal polycondensation method are urea, thiourea, melamine and dicyandiamide. The fabrication of $\mathrm{g}-\mathrm{C}_{3} \mathrm{~N}_{4}-\mathrm{BiOX} / \mathrm{Bi}_{\mathrm{x}} \mathrm{O}_{\mathrm{y}} \mathrm{X}_{\mathrm{z}}$ as $2 \mathrm{D}-2 \mathrm{D}$ heterojunction photocatalysts is usually achieved by growing $\mathrm{BiOX} / \mathrm{Bi}_{\mathrm{x}} \mathrm{O}_{\mathrm{y}} \mathrm{X}_{\mathrm{z}}$ on the surface of pre-synthesized $\mathrm{g}-\mathrm{C}_{3} \mathrm{~N}_{4}$. An overview of the various synthetic methods and the corresponding growth mechanism is presented in detail.

\subsection{In Situ Self-Assembly}

The effective use of electrostatic forces in the self-assembly and fabrication of nanostructures is gaining significance owing to their flexibility to work at room temperature and also due to their ability to offer rigid interface among the integrated components. For example, Yang et al. synthesized $\mathrm{BiOBr} / \mathrm{g}-\mathrm{C}_{3} \mathrm{~N}_{4}$ composite through the in situ self-assembly process based on electrostatic interaction between the precursors followed by their precipitation. In a typical process, pre-synthesized g- $\mathrm{C}_{3} \mathrm{~N}_{4}$ was protonated by treating it with $\mathrm{HCl}$ solution for converting its surface charge from negative to positive. The protonated g- $\mathrm{C}_{3} \mathrm{~N}_{4}$ was then added to $\mathrm{KBr}$ solution such that the $\mathrm{Br}^{-}$gets attracted to its surface and subsequently undergoes a precipitation reaction to form $\mathrm{BiOBr}$ with the addition of $\mathrm{Bi}\left(\mathrm{NO}_{3}\right)_{3} \bullet 5 \mathrm{H}_{2} \mathrm{O}$ solution [62]. Therefore, the $\mathrm{BiOBr}$ layer was favourably formed on the positively charged surface of g- $\mathrm{C}_{3} \mathrm{~N}_{4}$ and led to the formation of a tightly bound 2D-2D semiconductor heterojunction. Similarly, a p-n heterojunction between flower-like BiOI sheets and g- $\mathrm{C}_{3} \mathrm{~N}_{4}$ nanoparticles was constructed through an electrostatic self-assembly of g- $\mathrm{C}_{3} \mathrm{~N}_{4}$ nanoparticles, wherein the zeta potential of $\mathrm{BiOI}$ sheets was $-11.1 \mathrm{mV}$ and that of the $\mathrm{g}-\mathrm{C}_{3} \mathrm{~N}_{4}$ nanoparticles was $+21.5 \mathrm{mV}$ [75]. The measured values of zeta potential clearly indicated that the heterojunction formed between them was via the electrostatic self-assembly process.

\subsection{Hydrothermal and Solvothermal Synthesis}

Hydrothermal synthesis refers to process of heating water above its boiling point in a sealed reaction vessel to create supercritical fluid that in turn facilitates the precipitation or crystallization of inorganic materials under auto-generated pressure. The hydrothermal synthesis of nanostructured materials is similar to the processes governing the formation of minerals under the earth's crust that have been experimentally studied by geologists. The hydrothermal process can be used for dissolving and recrystallizing a substance that is poorly soluble or insoluble under normal conditions. Typically, an aqueous mixture of precursors sealed in a stainless steel autoclave heated above the boiling point of water results in the single-step production of highly crystalline materials due to the synergistic effect of high temperature and pressure [76]. The merits of hydrothermal synthesis are the enhanced crystallinity of synthesized materials without the need for further calcination, and easy control of the morphology and phase composition by controlling the temperature and reaction time. Under hydrothermal conditions, reactants enter the solution in the form of ions and are adsorbed, decomposed and desorbed at the growth interface before crystallizing. Solvothermal synthesis is analogous to the hydrothermal synthesis process, except for the fact that water is replaced by an organic solvent such as ethanol, ethylene glycol, etc. Adjusting the thermodynamic and kinetic parameters of the solvothermal 
synthesis reaction such as the concentration of the reactant precursors, reaction time, $\mathrm{pH}$ and temperature aids in controlling the size, shape, uniformity, dimensionality, phase and facets of the inorganic materials [77]. Therefore, the hydrothermal and solvothermal reactions can possibly ensure the intimate interface contact between $\mathrm{BiOX} / \mathrm{Bi}_{\mathrm{x}} \mathrm{O}_{\mathrm{y}} \mathrm{X}_{\mathrm{z}}$ and g- $\mathrm{C}_{3} \mathrm{~N}_{4}$ for promoting the rapid transport of photogenerated charge carriers across the interface.

Xiao et al. reported the synthesis of thirteen kinds of $\mathrm{BiOX}$ and $\mathrm{Bi}_{\mathrm{x}} \mathrm{O}_{\mathrm{y}} \mathrm{X}_{\mathrm{z}}$, viz., BiOI, $\mathrm{Bi}_{4} \mathrm{O}_{5} \mathrm{I}_{2}, \mathrm{Bi}_{7} \mathrm{O}_{9} \mathrm{I}_{3}, \mathrm{Bi}_{5} \mathrm{O}_{7} \mathrm{I}, \mathrm{BiOBr}, \mathrm{Bi}_{4} \mathrm{O}_{5} \mathrm{Br}_{2}, \mathrm{Bi}_{24} \mathrm{O}_{31} \mathrm{Br}_{10}, \mathrm{Bi}_{3} \mathrm{O}_{4} \mathrm{Br}, \mathrm{BiOCl}, \mathrm{Bi}_{12} \mathrm{O}_{15} \mathrm{Cl}_{6}$, $\mathrm{Bi}_{24} \mathrm{O}_{31} \mathrm{Cl}_{10}, \mathrm{Bi}_{3} \mathrm{O}_{4} \mathrm{Cl}$, and $\mathrm{Bi}_{12} \mathrm{O}_{17} \mathrm{Cl}_{2}$ through a general one-pot hydrothermal route by reacting different compositions of $\mathrm{Bi}_{2} \mathrm{O}_{3}$ and $\mathrm{KX}(\mathrm{X}=\mathrm{Cl}, \mathrm{Br}$ and $\mathrm{I})$ with nitric acid, and it was the first of its kind [78]. Since then, hydrothermal synthesis for the fabrication of $\mathrm{BiOX}$ and $\mathrm{Bi}_{\mathrm{x}} \mathrm{O}_{\mathrm{y}} \mathrm{X}_{\mathrm{z}}$ with various morphologies such as microspheres, microflowers, and microdisks (3D hierarchical structures) was achieved and comprised of three main growth steps: (i) the creation of $\mathrm{BiOX}$ nuclei, (ii) the growth of 2D nanosheets through the dissolution-renucleation process, and (iii) the formation of 3D nanostructures from the oriented attachment of 2D nanosheets under the influence of an electrostatic multipole field $[79,80]$. The hydrothermal method with L-lysine as a bio-template was employed in the fabrication of $\mathrm{BiOBr} / \mathrm{g}-\mathrm{C}_{3} \mathrm{~N}_{4}$ semiconductor heterojunction. Flake-like g- $\mathrm{C}_{3} \mathrm{~N}_{4}$ was pre-synthesized by the thermal polycondensation of melamine followed by sonochemical treatment in $\mathrm{NH}_{4} \mathrm{Cl}$ solution and subsequent sintering at $550{ }^{\circ} \mathrm{C}$. BiOBr microspheres with various mass ratios $\left(5,10,15,20\right.$ and $25 \%$ ) were grown in situ on flake-like g- $C_{3} \mathrm{~N}_{4}$ under hydrothermal conditions with $\mathrm{Bi}\left(\mathrm{NO}_{3}\right)_{3} \bullet 5 \mathrm{H}_{2} \mathrm{O}, \mathrm{NaBr}$ as precursors and L-lysine as the bio-template. Experimental investigation using TEM revealed that $\mathrm{BiOBr}$ microspheres synthesized with L-lysine as the template exhibited a loose structure with a larger percentage of exposed nanosheets that enhanced the amount of active sites for the degradation of organic pollutants in comparison to those synthesized without L-lysine [81]. Similarly, the hierarchical nanostructures of $\mathrm{Bi}_{\mathrm{x}} \mathrm{O}_{\mathrm{y}} \mathrm{X}_{\mathrm{z}}$ synthesized hydro/solvothermally with interconnected porous networks were reported to accelerate molecular diffusion/transport, enhance the overall light utilization efficiency, possess a large accessible surface area and provide better permeability, which could not only furnish adequate active adsorption sites and photocatalytic reaction sites, but also contributed to uniformly distributing the active sites in the fabricated photocatalysts [82]. The solvothermal method employed for synthesizing $\mathrm{Bi}_{5} \mathrm{O}_{7} \mathrm{Br}$ nanotubes using oleylamine as the solvent exhibited good visible light absorption and created oxygen vacancies on the surface that were beneficial for the stable photoreduction process [83]. Liu et al. reported the solvothermal synthesis of a 3D hierarchical structure of g- $\mathrm{C}_{3} \mathrm{~N}_{4} @ \mathrm{Bi} / \mathrm{BiOBr}$ with ternary heterojunction employing ethylene glycol as the solvent and reducing agent, which exhibited notably high photocatalytic activity for degrading organic pollutants [84]. Similarly, ethylene glycol assisted solvothermal synthesis reported by Ji et al. for the fabrication of ultrathin $\mathrm{Bi}_{4} \mathrm{O}_{5} \mathrm{Br}_{2}$ nanosheets dispersed over layered g- $\mathrm{C}_{3} \mathrm{~N}_{4}$ also exhibited higher photocatalytic activity for ciprofloxacin decomposition under visible light irradiation [85]. Another report on solvothermal synthesis was reported for the synthesis of $\mathrm{g}-\mathrm{C}_{3} \mathrm{~N}_{4} / \mathrm{I}^{3-}$-BiOI heterojunction semiconductor using self-stabilized $\mathrm{I}_{3}{ }^{-} / \mathrm{I}^{-}$as a redox mediator that efficiently strengthened the interaction between porous $\mathrm{g}-\mathrm{C}_{3} \mathrm{~N}_{4}$ and ultrathin $\mathrm{BiOI}$, thereby enhancing their photocatalytic activity in $\mathrm{CH}_{3} \mathrm{SH}$ oxidation [63].

\subsection{Ionic Liquid-Assited Method}

Solvent plays a prominent role in controlling the morphology of the nanostructured materials synthesized through the liquid phase synthesis techniques. Though organic solvents employed in various synthetic techniques are immensely useful in the shape and size controlled synthesis of nanostructured materials, some of their drawbacks such as poor solubility of inorganic precursors, low boiling point, high vapor pressure, high toxicity and flammable/explosive nature make them unpopular. Therefore, ionic liquids are gaining significant attention as a green medium for the synthesis of inorganic materials 
due to the growing environmental awareness. Low melting point, high chemical and thermal stability, high polarity for solubilizing a wide range of compounds, and ability to act as an ionic halide source are the attractive properties of ionic liquids. Various semiconductor photocatalysts have been synthesized using ionic liquid as solvent, and since they possess halogens in their functional groups, they are more suited to the preparation of $\mathrm{BiOX} / \mathrm{Bi}_{\mathrm{x}} \mathrm{O}_{\mathrm{y}} \mathrm{X}_{\mathrm{z}}$ [86]. For example, $\mathrm{Xia}$ et al. reported the synthesis of ultrathin g- $\mathrm{C}_{3} \mathrm{~N}_{4} / \mathrm{Bi}_{4} \mathrm{O}_{5} \mathrm{I}_{2}$ layered nanojunctions using [Hmim]I (1-hexyl-3-methylimidazolium iodide) ionic liquid. Highly reactive ionic liquid acted as the iodine source, also served as the capping agent for the formation of ultrasmall $\mathrm{Bi}_{4} \mathrm{O}_{5} \mathrm{I}_{2}$ nanosheets and facilitated the wide distribution over ultrathin g- $\mathrm{C}_{3} \mathrm{~N}_{4}$. The growth of ultrasmall $\mathrm{Bi}_{4} \mathrm{O}_{5} \mathrm{I}_{2}$ and their wide distribution over the ultrathin $\mathrm{g}-\mathrm{C}_{3} \mathrm{~N}_{4}$ promoted the construction of a tight heterojunction under hydrothermal conditions [87].

Similarly, g- $\mathrm{C}_{3} \mathrm{~N}_{4} / \mathrm{BiOBr}$ microspheres were synthesized by the dispersion of g- $\mathrm{C}_{3} \mathrm{~N}_{4}$ to a solution made by dissolving $\mathrm{Bi}\left(\mathrm{NO}_{3}\right)_{3} \bullet 5 \mathrm{H}_{2} \mathrm{O}$ in ethanol containing a stoichiometric amount of ionic liquid [ $\left.\mathrm{C}_{16} \mathrm{mim}\right] \mathrm{Br}$ (1-hexadecyl-3-methylimidazolium bromide). During the reaction, the ionic liquid $\left[\mathrm{C}_{16} \mathrm{mim}\right] \mathrm{Br}$ acted as the solvent, reactant, template and most importantly as a dispersing agent, which ensured the better dispersion of g- $\mathrm{C}_{3} \mathrm{~N}_{4}$ in the aqueous solution due to electrostatic attraction. As observed from Figure 4, the FESEM and TEM micrographs of the solvothermally synthesized g- $\mathrm{C}_{3} \mathrm{~N}_{4} / \mathrm{BiOBr}$ composites exhibited relatively uniform $3 \mathrm{D}$ flower-like microspheres with self-assembled nanosheets on their surface, indicating the wide distribution of $\mathrm{g}^{-} \mathrm{C}_{3} \mathrm{~N}_{4}$ on the surface of $\mathrm{BiOBr}[88,89]$.
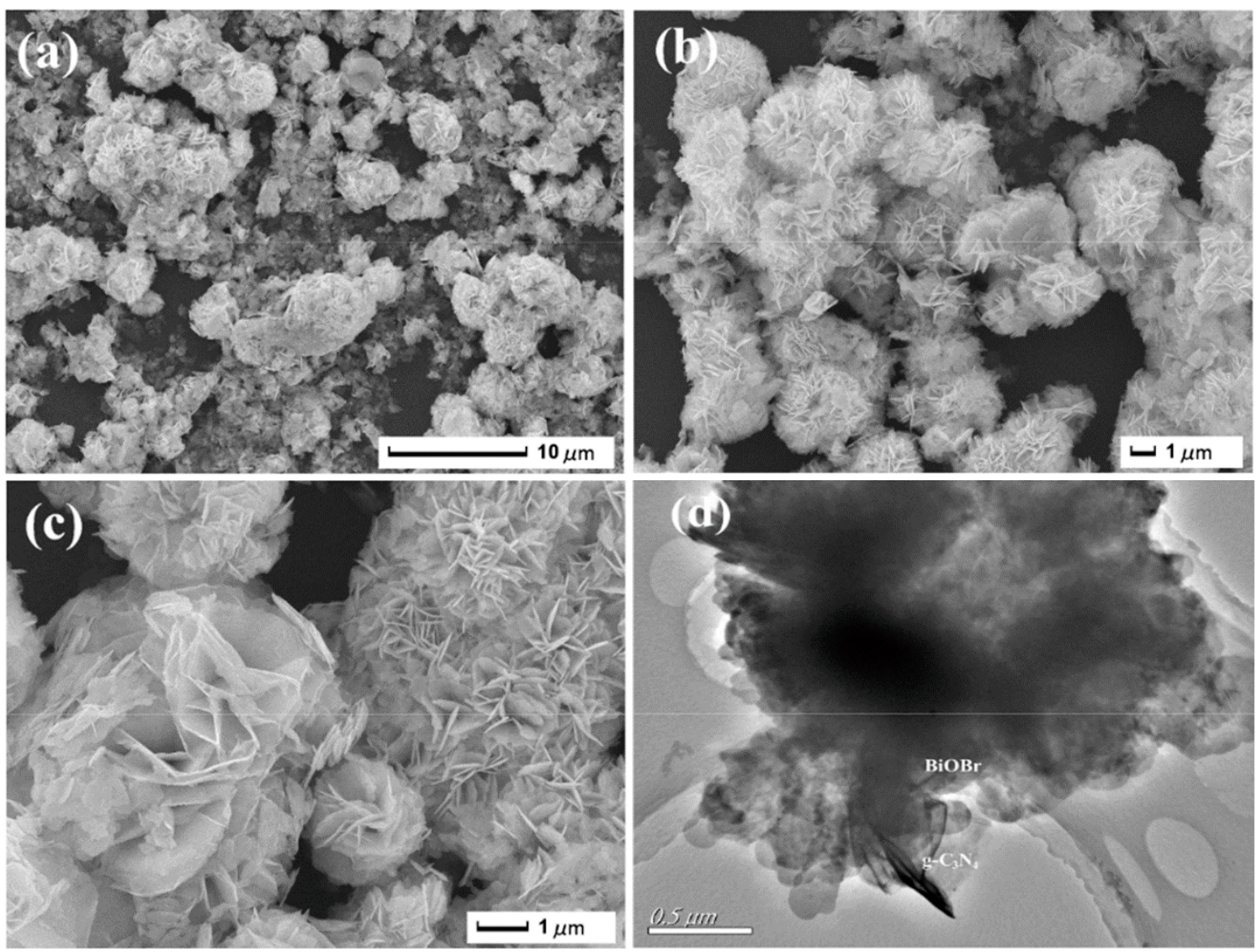

Figure 4. (a) Low magnification and (b,c) magnified FESEM and (d) TEM micrographs of g- $\mathrm{C}_{3} \mathrm{~N}_{4} / \mathrm{BiOBr}$ microspherical composites synthesized by ionic liquid assisted solvothermal method. Reprinted from Ref. [88] with permission from The Royal Society of Chemistry. 


\subsection{Precipitation Technique}

Precipitation is a simple, cost-effective and rapid process of synthesizing semiconductor photocatalysts that can be easily replicated on a larger scale for industrial applications. Further, it is an eco-friendly route that hardly requires any hazardous organic solvents and treatments under high pressure or temperature [90]. The precipitation synthesis of $\mathrm{BiOX}$ typically involves the dropwise addition of halide $(\mathrm{KX}$ or $\mathrm{NaX}$, where $\mathrm{X}=\mathrm{Cl}, \mathrm{Br}$ and I) solution into a solution of bismuth salt $\left(\mathrm{BiCl}_{3}, \mathrm{Bi}\left(\mathrm{NO}_{3}\right)_{3}\right.$ or $\left.\mathrm{Bi}_{2} \mathrm{O}_{3}\right)$ under acidic conditions. For instance, Ren et al. reported the preparation of three series of $\mathrm{BiOM}_{\mathrm{x}} \mathrm{R}_{1-\mathrm{x}}(\mathrm{M}, \mathrm{R}=\mathrm{Cl}, \mathrm{Br}, \mathrm{I})$ solid solutions with 3D nanostructured morphology and adjustable bandgap energy through a low-temperature precipitation technique [59]. Appropriately, adjusting the amount of solute and the solvent in the solid solutions led to the formation of $\mathrm{BiOM}_{\mathrm{x}} \mathrm{R}_{1-\mathrm{x}}$ photocatalysts that could absorb visible light in the range 359-675 $\mathrm{nm}$ with a bandgap energy ranging from 3.3-1.7 eV. Composite heterojunctions of $\mathrm{g}-\mathrm{C}_{3} \mathrm{~N}_{4} / \mathrm{Bi}_{12} \mathrm{O}_{17} \mathrm{Cl}_{2}$ were prepared by dispersing pre-synthesized $\mathrm{g}-\mathrm{C}_{3} \mathrm{~N}_{4}$ into an ethanol solution of $\mathrm{BiCl}_{3}$ at $\mathrm{pH} 2$. The dropwise addition of freshly prepared aqueous $\mathrm{NaOH}$ solution into the ethanol solution containing the mixture led to the formation of $\mathrm{g}-\mathrm{C}_{3} \mathrm{~N}_{4} / \mathrm{Bi}_{12} \mathrm{O}_{17} \mathrm{Cl}_{2}$ while the $\mathrm{pH}$ reached 14 [60]. Chen et al. reported the synthesis of hierarchical hexagonal plates of $\mathrm{Bi}_{24} \mathrm{O}_{31} \mathrm{Br}_{10}$ through co-precipitation and subsequent solvothermal treatment in ethylene glycol, which produced a hierarchical structure by the process of dissolution-recrystallization of $1 \mathrm{D}$ $\mathrm{Bi}_{24} \mathrm{O}_{31} \mathrm{Br}_{10}$ nanobelts [61]. In another study, a BiOI-BiOCl/g- $\mathrm{C}_{3} \mathrm{~N}_{4}$ ternary composite was synthesized by a template-free precipitation method using $\mathrm{NH}_{3}$ solution as the precipitating agent, wherein thin layers of $\mathrm{g}-\mathrm{C}_{3} \mathrm{~N}_{4}$ acted as a bed for anchoring $\mathrm{BiOI}$ and $\mathrm{BiOCl}$ nanosheets for the formation of an efficient heterojunction semiconductor [91].

\subsection{Reflux Process}

Reflux based synthesis is based on the thermal energy supplied for the progress of the reaction over long periods of time. The phase and morphology of the synthesized nanostructured materials are directly dependent on parameters, viz., the order in which the precursors are added, reflux time and cooling rate [47]. Mousavi et al. employed the reflux technique for the fabrication of $\mathrm{g}-\mathrm{C}_{3} \mathrm{~N}_{4} / \mathrm{Fe}_{3} \mathrm{O}_{4} / \mathrm{BiOI}$ nanocomposites. As the first step, $\mathrm{Fe}_{3} \mathrm{O}_{4}$ nanoparticles were deposited on the surface of pre-synthesized $\mathrm{g}-\mathrm{C}_{3} \mathrm{~N}_{4}$ to form $\mathrm{g}-\mathrm{C}_{3} \mathrm{~N}_{4} / \mathrm{Fe}_{3} \mathrm{O}_{4}$. Next, $\mathrm{BiOI}$ was synthesized over the surface of $\mathrm{g}-\mathrm{C}_{3} \mathrm{~N}_{4} / \mathrm{Fe}_{3} \mathrm{O}_{4}$ by a precipitation reaction between $\mathrm{Bi}\left(\mathrm{NO}_{3}\right)_{3} \bullet 5 \mathrm{H}_{2} \mathrm{O}$ and $\mathrm{NaI}$, followed by refluxing for $30 \mathrm{~min}$ at $96{ }^{\circ} \mathrm{C}$ [92]. Similarly, the fabrication of g- $\mathrm{C}_{3} \mathrm{~N}_{4} /$ carbon dots/ $\mathrm{BiOCl}$ and g- $\mathrm{C}_{3} \mathrm{~N}_{4} /$ carbon dots $/ \mathrm{BiOBr}$ heterojunction photocatalysts was also reported by employing the reflux process $[93,94]$.

\subsection{Solid-State Calcination}

Solid-state calcination is a viable method for the preparation of materials without the utilization of water. Weak van der Waals interaction existing between halogen atoms results in the phase transition from $\mathrm{BiOX}$ to $\mathrm{Bi}_{\mathrm{x}} \mathrm{O}_{\mathrm{y}} \mathrm{X}_{\mathrm{z}}$ during the process of calcination due to the removal of unstable halogen. Therefore, $\mathrm{Bi}_{\mathrm{x}} \mathrm{O}_{\mathrm{y}} \mathrm{X}_{\mathrm{z}}$ materials are prepared by the high temperature treatment of the precursors mixed with appropriate stoichiometric ratio. For example, $\mathrm{Di}$ et al. reported the preparation of $\mathrm{Bi}_{12} \mathrm{O}_{17} \mathrm{Cl}_{2}$ by calcining a mixture of $\mathrm{Bi}_{2} \mathrm{O}_{3}$ and $\mathrm{BiOCl}$ in stoichiometric proportions at $650{ }^{\circ} \mathrm{C}$ for $10 \mathrm{~h} \mathrm{[65].} \mathrm{A} \mathrm{similar} \mathrm{process}$ was reported for synthesizing $\mathrm{Bi}_{3} \mathrm{O}_{4} \mathrm{Br}$ by the calcination of $\mathrm{Bi}_{2} \mathrm{O}_{3}$ and $\mathrm{BiOBr}$ mixture at $650{ }^{\circ} \mathrm{C}$ for $10 \mathrm{~h}$ [95]. Additionally, the solid-state calcination method was employed in the fabrication of $\mathrm{Bi}_{3} \mathrm{O}_{4} \mathrm{Cl} / \mathrm{g}-\mathrm{C}_{3} \mathrm{~N}_{4}$ heterojunction that was reported to have a tight face-to-face connection between the semiconductors for improved photocatalytic activity [96].

\subsection{Sonochemical Synthesis}

Sonochemical (also known as ultrasound-assisted) synthesis is a versatile approach that utilizes the high intensity ultrasound for the production of nanostructured inorganic materials in a controllable fashion, which are often unattainable through the conventional 
methods [97]. Water and ionic liquids are typically used as replacements for volatile and toxic organic solvents [98]. In comparison to the chemical reactions progressing through the supply of common energy sources (such as heat, light, electric potential, radiation, etc.), the ultrasonic irradiation provides an unusual reaction condition that leads to acoustic cavitation (i.e., the formation, growth and implosive collapse of bubbles in liquids), which drives the rapid nucleation and growth of the inorganic materials. For example, Liu et al. reported the synthesis of a g- $\mathrm{C}_{3} \mathrm{~N}_{4} / \mathrm{BiOBr}$ heterojunction photocatalyst through the sonochemical synthesis technique [99]. A solution of $\mathrm{Bi}\left(\mathrm{NO}_{3}\right)_{3} \bullet 5 \mathrm{H}_{2} \mathrm{O}$ dissolved in ethylene glycol was mixed with DI water containing pre-synthesized $\mathrm{g}-\mathrm{C}_{3} \mathrm{~N}_{4}$ under ultrasound irradiation at $40{ }^{\circ} \mathrm{C}$ for $2 \mathrm{~h}$ to form a uniform suspension, to which a stoichiometric proportion of $\mathrm{NaBr}$ and PVP were added dropwise and heated to $80^{\circ} \mathrm{C}$ for $3 \mathrm{~h}$. TEM micrographs of the pristine $\mathrm{g}-\mathrm{C}_{3} \mathrm{~N}_{4}$, pristine $\mathrm{BiOBr}$ and sonochemically synthesized g- $\mathrm{C}_{3} \mathrm{~N}_{4} / \mathrm{BiOBr}$ are shown in Figure 5. A schematic representation of the 2D-2D heterojunction (Figure 5g) and the elemental maps (Figure $5 \mathrm{~h}$ ) confirming the deposition of $\mathrm{BiOBr}$ over $\mathrm{g}-\mathrm{C}_{3} \mathrm{~N}_{4}$ is also shown in Figure 5.

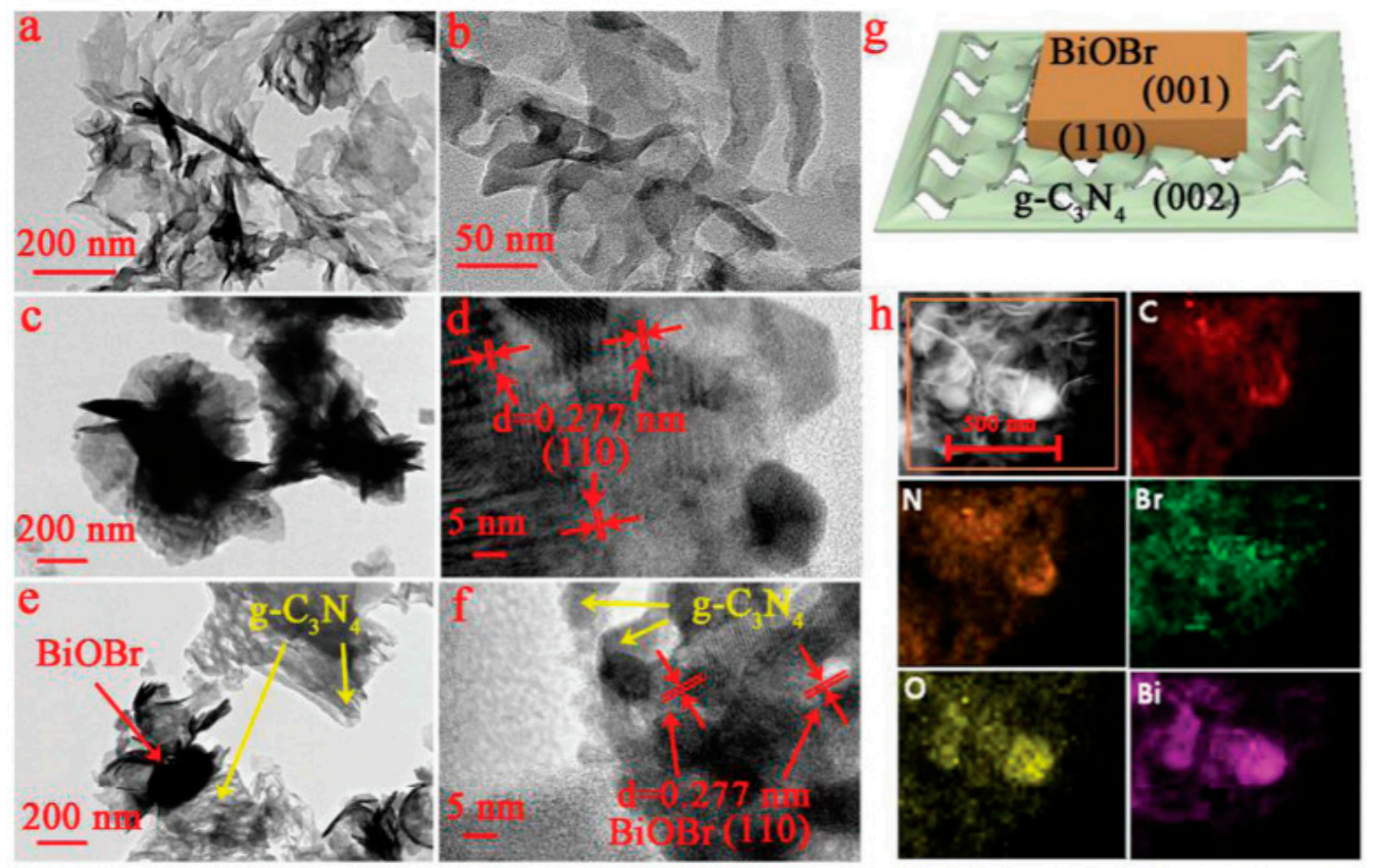

Figure 5. TEM micrographs of $(\mathbf{a}, \mathbf{b})$ pristine $g-\mathrm{C}_{3} \mathrm{~N}_{4},(\mathbf{c}, \mathbf{d})$ pristine $\mathrm{BiOBr}$ and $(\mathbf{e}, \mathbf{f}) \mathrm{g}-\mathrm{C}_{3} \mathrm{~N}_{4} / \mathrm{BiOBr}$; (g) schematic representation of growth of $\mathrm{BiOBr}$ layer over the surface of $\mathrm{g}-\mathrm{C}_{3} \mathrm{~N}_{4}$ forming the heterojunction photocatalyst and (h) elemental maps confirming the growth of $\mathrm{BiOBr}$ over $\mathrm{g}-\mathrm{C}_{3} \mathrm{~N}_{4}$. Reprinted from Ref. [99] with permission from The Royal Society of Chemistry.

\section{Photocatalytic Activity}

\subsection{Photocatalytic Degradation of Organic and Inorganic Contaminats}

$\mathrm{BiOX} / \mathrm{Bi}_{\mathrm{x}} \mathrm{O}_{\mathrm{y}} \mathrm{X}_{\mathrm{z}}$ photocatalysts have demonstrated admirable performance in the degradation of various organic and inorganic contaminants such as methyl orange, rhodamine B, methylene blue, acid orange, microcystin-LR, 2,4 dichlorophenol, bisphenol-A, tetracycline hydrochloride, phenol, carbamazepine, levofloxacin, metronidazole, fuchsine, methyl mercapton, sulfamethoxazole, mercury, chromium, etc. In general, the photocatalytic reaction for the degradation of organic contaminants involves three simultaneous steps, viz., photoexcitation for the generation of charge carriers $\left(\mathrm{e}^{-}\right.$and $\left.\mathrm{h}^{+}\right)$at the $\mathrm{CB}$ and $\mathrm{VB}$, the separation of charges and their transfer to the active sites on the semiconductor surface, the formation of radical species by the ionization of water, i.e., reaction of the holes $\left(\mathrm{h}^{+}\right)$with hydroxyl ions $\left(\mathrm{OH}^{-}\right)$to produce hydroxyl radicals $\left({ }^{\bullet} \mathrm{OH}\right)$ and reaction of the electrons $\left(\mathrm{e}^{-}\right)$with the superoxide anion radicals $\left({ }^{\circ} \mathrm{O}_{2}{ }^{-}\right)$, which subsequently react with 
the organic contaminants adsorbed on the photocatalyst surface [100,101]. Di et al. synthesized ultrathin $\mathrm{Bi}_{4} \mathrm{O}_{5} \mathrm{Br}_{2}$ and $\mathrm{BiOBr}$ nanosheets and studied their capability to degrade ciprofloxacin under visible light. Lower energy bandgap $(2.33 \mathrm{eV})$ and a more negative $C B$ position of ultrathin $\mathrm{Bi}_{4} \mathrm{O}_{5} \mathrm{Br}_{2}$ nanosheets facilitated the improved electronic transition, the generation of extra charge carriers and the formation of more ${ }^{\bullet} \mathrm{O}_{2}{ }^{-}$radicals that collectively enabled it to display a maximum rate constant of $0.0113 \mathrm{~min}^{-1}$, which was 1.9 times higher than ultrathin $\mathrm{BiOBr}$ nanosheets [102]. Wang et al. synthesized $\mathrm{Bi}_{24} \mathrm{O}_{31} \mathrm{Br}_{10}$ nanosheets with thicknesses of 40, 85 and $130 \mathrm{~nm}$ through the solvothermal method and utilized them for the photodegradation of tetracycline hydrochloride under visible light irradiation. The three $\mathrm{Bi}_{24} \mathrm{O}_{31} \mathrm{Br}_{10}$ nanosheets with $40 \mathrm{~nm}$ thickness demonstrated $95 \%$ degradation of tetracycline hydrochloride within $90 \mathrm{~min}$, in comparison to the thicker counterparts. The enhanced photocatalytic activity of $\mathrm{Bi}_{24} \mathrm{O}_{31} \mathrm{Br}_{10}$ nanosheets with $40 \mathrm{~nm}$ thickness was attributed to lattice defects formed by bromine vacancies that subsequently improved the charge carrier density, charge separation and transportation [103]. A BiOBr-g- $\mathrm{C}_{3} \mathrm{~N}_{4}$ heterojunction photocatalyst synthesized through a single-step chemical bath method exhibited enhanced photodegradation of $10 \mathrm{ppm}$ rhodamine $\mathrm{B}$ under visible light in comparison to pristine g- $\mathrm{C}_{3} \mathrm{~N}_{4}$, pristine $\mathrm{BiOBr}$ and a composite formed by mixing $\mathrm{g}-\mathrm{C}_{3} \mathrm{~N}_{4}$ and $\mathrm{BiOBr}$ in 1:1 weight ratio. The enhanced performance of the BiOBr-g- $\mathrm{C}_{3} \mathrm{~N}_{4}$ photocatalyst was attributed to the perfect coupling between the BiOBr- $\{001\}$ and g- $\mathrm{C}_{3} \mathrm{~N}_{4}-\{002\}$ facets, which facilitated the unhindered transport of the photogenerated charges while curbing their recombination [104]. Sphere-like g- $\mathrm{C}_{3} \mathrm{~N}_{4} / \mathrm{BiOI}$ composite photocatalysts synthesized using ionic liquids exhibited excellent photocatalytic activity in the degradation of rhodamine $\mathrm{B}$, methylene blue, methyl orange, bisphenol $\mathrm{A}$ and 4-chlorophenol under visible light irradiation. Among the various composite photocatalysts, the $15 \mathrm{wt} \% \mathrm{~g}-\mathrm{C}_{3} \mathrm{~N}_{4} / \mathrm{BiOI}$ exhibited optimal performance in comparison to pristine BiOI, which was attributed to the heterojunction formed between $\mathrm{g}-\mathrm{C}_{3} \mathrm{~N}_{4}$ and $\mathrm{BiOI}$ that effectively separated the photogenerated charge carriers and enhanced the interfacial charge transfer as evidenced through its photocurrent response [105]. Liu et al. reported the fabrication of g- $\mathrm{C}_{3} \mathrm{~N}_{4} / \mathrm{Bi}_{5} \mathrm{O}_{7} \mathrm{I}$ composite photocatalysts by the thermolysis of melamine with pre-synthesized $\mathrm{BiOI}$ at 520 ${ }^{\circ} \mathrm{C}$ for $4 \mathrm{~h}$ [106]. Interestingly, during thermolysis $\mathrm{BiOI}$ was transformed to $\mathrm{Bi}_{5} \mathrm{O}_{7} \mathrm{I}$ and a strong interfacial contact was established with $\mathrm{g}-\mathrm{C}_{3} \mathrm{~N}_{4}$ due to in situ co-crystallization, which enabled it to exhibit excellent performance in the photodegradation of rhodamine $B$ and phenol under visible light irradiation due to faster charge migration and separation over the heterojunction. The results revealed that $\mathrm{h}^{+}$and ${ }^{\bullet} \mathrm{O}_{2}{ }^{-}$were the primary active species, and the rate of photodegradation of rhodamine $\mathrm{B}$ using $30 \mathrm{wt} \% \mathrm{~g}-\mathrm{C}_{3} \mathrm{~N}_{4} / \mathrm{Bi}_{5} \mathrm{O}_{7} \mathrm{I}$ at $1.12 \mathrm{~h}^{-1}$ was $\sim 15$ and 3 times higher than that of pristine g- $\mathrm{C}_{3} \mathrm{~N}_{4}$ and $\mathrm{Bi}_{5} \mathrm{O}_{7} \mathrm{I}$, respectively. In another study, microspheres of $\mathrm{g}_{-} \mathrm{C}_{3} \mathrm{~N}_{4} / \mathrm{Bi}_{5} \mathrm{O}_{7} \mathrm{I}$ synthesized through the hydrothermal method using ethylene glycol as the solvent exhibited enhanced photodegradation of methyl orange and rhodamine $B$ with rate constants $0.084 \mathrm{~min}^{-1}$ and $0.197 \mathrm{~min}^{-1}$, respectively. The results of scavenger studies and electron spin resonance spectroscopy confirmed that ${ }^{\bullet} \mathrm{O}_{2}{ }^{-}$was the primary active species, which could only have been generated if the transfer mechanism was based on the Z-scheme heterojunction [107]. The visible light photocatalytic oxidation of hazardous gas-phase mercury $\left(\mathrm{Hg}^{0}\right)$ to divalent mercury $\left(\mathrm{Hg}^{2+}\right)$ for its easy removal was reported using $\mathrm{g}-\mathrm{C}_{3} \mathrm{~N}_{4} / \mathrm{Bi}_{5} \mathrm{O}_{7} \mathrm{I}$ nanosheets doped with $\mathrm{Yb}^{3+}$ [108]. As observed from Figure $6 \mathrm{a}$, the mercury removal efficiency of g- $\mathrm{C}_{3} \mathrm{~N}_{4} / \mathrm{Bi}_{5} \mathrm{O}_{7} \mathrm{I}$ doped with $\mathrm{Yb}^{3+}$ was $79.01 \%$ and $42.02 \%$, respectively, under visible and near infrared light radiation, while the efficiency under near infrared light was just $13.3 \%$ without $\mathrm{Yb}^{3+}$ doping. Scavenger studies and electron spin resonance spectroscopy revealed that ${ }^{\bullet} \mathrm{O}_{2}{ }^{-}$and ${ }^{\bullet} \mathrm{OH}$ were the primary active species responsible for the oxidation of gas phase $\mathrm{Hg}^{0}$, while the mechanism of charge transfer was based on the Z-scheme heterojunction with enhanced separation of electrons due to the formation of a new energy band below the $\mathrm{CB}$ of $\mathrm{Bi}_{5} \mathrm{O}_{7} \mathrm{I}$ as a result of doping $\mathrm{Yb}^{3+}$, as depicted in Figure $6 \mathrm{~b}$. 

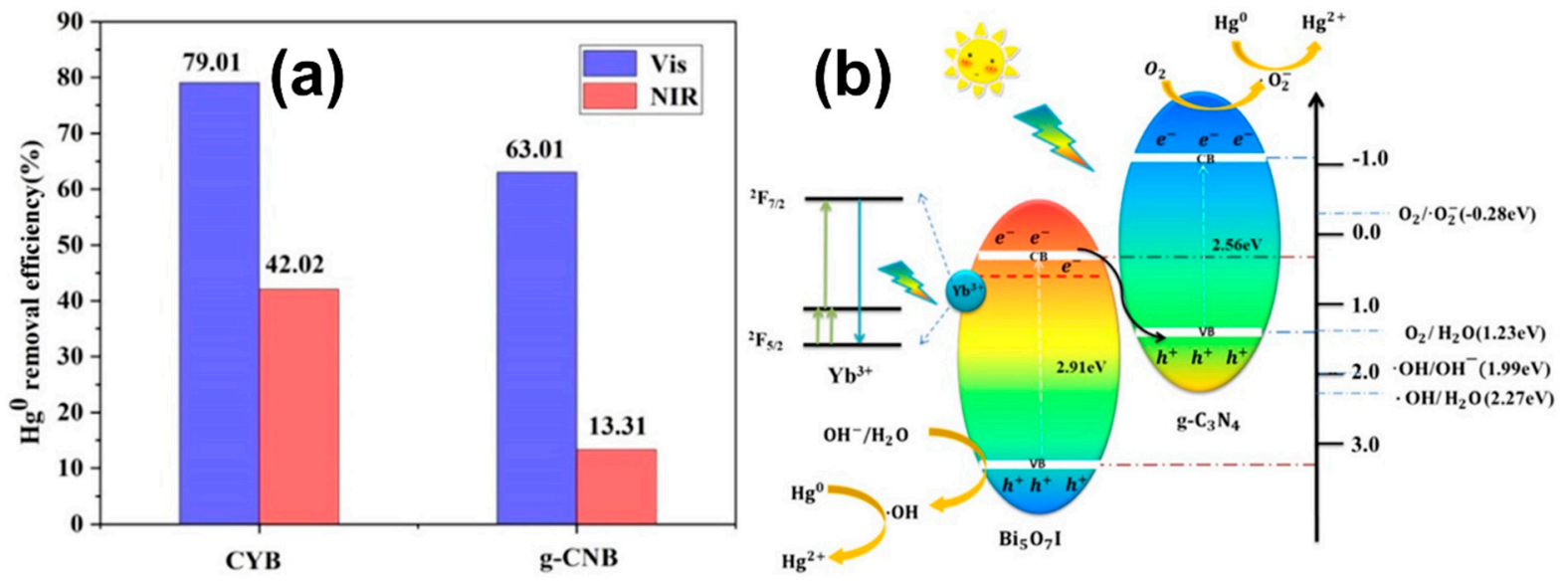

Figure 6. (a) Mercury removal efficiency under visible and near infrared light excitation in the presence of $g-\mathrm{C}_{3} \mathrm{~N}_{4} / \mathrm{Yb}^{3+}$ $\mathrm{Bi}_{5} \mathrm{O}_{7} \mathrm{I}(\mathrm{CYB})$ and $g-\mathrm{C}_{3} \mathrm{~N}_{4}-\mathrm{Bi}_{5} \mathrm{O}_{7} \mathrm{I}(\mathrm{g}-\mathrm{CNB})$ as photocatalysts, (b) schematic depicting the mechanism of charge transfer in the Z-scheme heterojunction $\mathrm{g}_{-} \mathrm{C}_{3} \mathrm{~N}_{4} / \mathrm{Yb}^{3+}-\mathrm{Bi}_{5} \mathrm{O}_{7} \mathrm{I}$ during the photocatalytic oxidation of $\mathrm{Hg}^{0}$. Reprinted from Ref. [108] with permission from American Chemical Society.

Zhang et al. reported the fabrication of a heterojunction photocatalyst by the in situ hydrothermal growth of $\mathrm{Bi}_{7} \mathrm{O}_{9} \mathrm{I}_{3}$ on ultrathin $\mathrm{g}-\mathrm{C}_{3} \mathrm{~N}_{4}$ for the degradation of doxycycline hydrochloride under visible light. Microspheres of $\mathrm{Bi}_{7} \mathrm{O}_{9} \mathrm{I}_{3} / g-\mathrm{C}_{3} \mathrm{~N}_{4}$ exhibited a photodegradation efficiency of $\sim 80 \%$ that was $\sim 2$ and 5.4 times greater than pristine $\mathrm{Bi}_{7} \mathrm{O}_{9} \mathrm{I}_{3}$ and $g-\mathrm{C}_{3} \mathrm{~N}_{4}$, respectively, which could be attributed to their large surface area $\left(68.55 \mathrm{~m}^{2} \mathrm{~g}^{-1}\right)$ and enhanced charge generation/separation in the heterojunction. Scavenger studies and electron spin resonance spectroscopy revealed that ${ }^{\bullet} \mathrm{O}_{2}{ }^{-}$and ${ }^{\bullet} \mathrm{OH}$ were the primary active species that were predominantly involved in breaking the stable structure of doxycycline hydrochloride, while all the experimental data and characterization evidence confirmed that the mechanism of photodegradation followed direct Z-scheme heterojunction [109]. In another study, a g- $\mathrm{C}_{3} \mathrm{~N}_{4}$ modified $\mathrm{Bi}_{4} \mathrm{O}_{5} \mathrm{I}_{2}$ composite prepared in situ by the thermal treatment of a g- $\mathrm{C}_{3} \mathrm{~N}_{4} / \mathrm{Bi}_{4} \mathrm{O}_{5} \mathrm{I}_{2}$ precursor at $400{ }^{\circ} \mathrm{C}$ for $3 \mathrm{~h}$ exhibited enhanced photocatalytic performance in the degradation of methyl orange under visible light with a degradation rate of $0.164 \mathrm{~min}^{-1}$, which was 3.2 and 82 times enhanced in comparison to pristine $\mathrm{Bi}_{4} \mathrm{O}_{5} \mathrm{I}_{2}$ and g- $\mathrm{C}_{3} \mathrm{~N}_{4}$, respectively [110]. A summary of the typical synthesis methods and photocatalytic performance of $\mathrm{BiOX} / \mathrm{Bi}_{\mathrm{x}} \mathrm{O}_{\mathrm{y}} \mathrm{X}_{\mathrm{z}}-\mathrm{g}-\mathrm{C}_{3} \mathrm{~N}_{4}$ heterojunction photocatalysts involved in the degradation of various organic pollutants is presented in Table 1. Further, the details corresponding to the mechanism of photogenerated charge transfer during the degradation of organic pollutants are briefly explained in Section 5.5.

Table 1. Summary of the degradation of organic contaminants in the presence of $\mathrm{BiOX} / \mathrm{Bi}_{\mathrm{x}} \mathrm{O}_{\mathrm{y}} \mathrm{X}_{\mathrm{z}}-\mathrm{g}-\mathrm{C}_{3} \mathrm{~N}_{4}$ heterojunction photocatalysts reported in the literature.

\begin{tabular}{|c|c|c|c|c|c|c|c|}
\hline $\begin{array}{l}\text { Synthesis } \\
\text { Method }\end{array}$ & Precursors & Morphology & $\begin{array}{l}\text { Contaminant } \\
\text { Parameters }\end{array}$ & Light Source & $\begin{array}{l}\text { Heterojunction } \\
\text { Type }\end{array}$ & $\begin{array}{c}\text { Significance of the } \\
\text { Result }\end{array}$ & Ref. \\
\hline \multicolumn{8}{|c|}{$\mathrm{BiOX}-\mathrm{g}-\mathrm{C}_{3} \mathrm{~N}_{4}$} \\
\hline \multicolumn{8}{|c|}{ BiOI-g- $\mathrm{C}_{3} \mathrm{~N}_{4}$} \\
\hline $\begin{array}{l}\text { Solid-phase } \\
\text { calcination }\end{array}$ & $\begin{array}{l}\mathrm{Bi}\left(\mathrm{NO}_{3}\right)_{3} \cdot 5 \mathrm{H}_{2} \mathrm{O}, \mathrm{KI} \\
\quad \text { and } \mathrm{C}_{3} \mathrm{H}_{6} \mathrm{~N}_{6}\end{array}$ & $\begin{array}{c}\text { Layers of } \mathrm{g}^{-} \mathrm{C}_{3} \mathrm{~N}_{4} \\
\text { grown on the } \\
\text { surface of } \mathrm{BiOI} \\
\text { microspheres }\end{array}$ & $\begin{array}{l}\text { Microcystin- } \\
\text { LR (5 } \\
\text { ppm) }\end{array}$ & $\begin{array}{c}350 \text { W Xe lamp } \\
(\lambda>420 \mathrm{~nm})\end{array}$ & $\begin{array}{c}\text { Direct } \\
\text { solid-state } \\
\text { Z-scheme }\end{array}$ & $\begin{array}{l}\text { Optimized content } \\
\text { of g- } \mathrm{C}_{3} \mathrm{~N}_{4} \text { over } \\
\text { BiOI for high } \\
\text { activity was found } \\
\text { to be } 4 \mathrm{wt} \%\end{array}$ & [111] \\
\hline $\begin{array}{l}\text { Electrostatic } \\
\text { self- } \\
\text { assembly }\end{array}$ & $\begin{array}{c}\mathrm{Bi}\left(\mathrm{NO}_{3}\right)_{3} \cdot 5 \mathrm{H}_{2} \mathrm{O}, \mathrm{KI}, \\
\mathrm{C}_{3} \mathrm{H}_{6} \mathrm{~N}_{6}, \mathrm{H}_{2} \mathrm{SO}_{4} \\
\mathrm{HNO}_{3} \text {, and } \\
\mathrm{C}_{2} \mathrm{H}_{6} \mathrm{O}_{2}\end{array}$ & $\begin{array}{c}\mathrm{g}-\mathrm{C}_{3} \mathrm{~N}_{4} \\
\text { nanoparticles on } \\
\text { flower-like BiOI } \\
\text { nanosheets }\end{array}$ & $\begin{array}{l}\text { Methyl orange } \\
\text { (10 ppm) }\end{array}$ & $\begin{array}{c}300 \mathrm{~W} \text { Xe lamp } \\
(\lambda>420 \mathrm{~nm})\end{array}$ & $\mathrm{p}-\mathrm{n}$ & $\begin{array}{l}\text { Surficial dispersive } \\
\text { heterojunctions } \\
\text { were beneficial for } \\
\text { degradation of } \mathrm{MO}\end{array}$ & [75] \\
\hline
\end{tabular}


Table 1. Cont.

\begin{tabular}{|c|c|c|c|c|c|c|c|}
\hline $\begin{array}{l}\text { Synthesis } \\
\text { Method }\end{array}$ & Precursors & Morphology & $\begin{array}{l}\text { Contaminant } \\
\text { Parameters }\end{array}$ & Light Source & $\begin{array}{c}\text { Heterojunction } \\
\text { Type }\end{array}$ & $\begin{array}{c}\text { Significance of the } \\
\text { Result }\end{array}$ & Ref. \\
\hline $\begin{array}{c}\text { Simple } \\
\text { precipitation }\end{array}$ & $\begin{array}{c}\mathrm{Bi}\left(\mathrm{NO}_{3}\right)_{3} \cdot 5 \mathrm{H}_{2} \mathrm{O}, \mathrm{KI} \\
\mathrm{C}_{3} \mathrm{H}_{6} \mathrm{~N}_{6}, \mathrm{C}_{2} \mathrm{H}_{6} \mathrm{O}_{2} \\
\text { and CTAB }\end{array}$ & $\begin{array}{l}\text { Thin nanosheets of } \\
\text { BiOI lie on the } \\
\text { surface of } g-C_{3} N_{4}\end{array}$ & $\begin{array}{c}2,4- \\
\text { dichlorophenol } \\
\text { (10 ppm) } \\
\text { Bisphenol A } \\
\text { (10 ppm) } \\
\text { Rhodamine B } \\
\text { (100 ppm) } \\
\text { Tetracycline } \\
\text { hydrochloride } \\
\text { (10 ppm) }\end{array}$ & $\begin{array}{c}300 \mathrm{~W} \text { Xe lamp } \\
(\lambda>420 \mathrm{~nm})\end{array}$ & $\mathrm{p}-\mathrm{n}$ & $\begin{array}{c}\text { Top-top facets of } \\
\text { BiOI }(001) / g-\mathrm{C}_{3} \mathrm{~N}_{4} \\
(002) \text { promoted } \\
\text { generation of }{ }^{1} \mathrm{O}_{2} \\
\text { and }{ }^{\bullet} \mathrm{O}_{2}{ }^{-} \\
\text {accounting for } \\
\text { excellent } \\
\text { photocatalytic } \\
\text { activity }\end{array}$ & [112] \\
\hline $\begin{array}{l}\text { In situ } \\
\text { precursor } \\
\text { transforma- } \\
\text { tion }\end{array}$ & $\begin{array}{c}\mathrm{CO}\left(\mathrm{NH}_{2}\right)_{2} \\
\mathrm{Bi}\left(\mathrm{NO}_{3}\right)_{3} \cdot 5 \mathrm{H}_{2} \mathrm{O}, \mathrm{KI} \\
\text { and } \mathrm{C}_{2} \mathrm{H}_{6} \mathrm{O}_{2}\end{array}$ & $\begin{array}{c}\text { Numerous } \\
\text { quantum-sized } \\
\text { nanoparticles are } \\
\text { uniformly } \\
\text { dispersed across } \\
\text { the g- } \mathrm{C}_{3} \mathrm{~N}_{4} \\
\text { nanosheets }\end{array}$ & $\begin{array}{l}\text { Phenol (100 } \\
\text { ppm) }\end{array}$ & $\begin{array}{c}60 \mathrm{~W} \text { LED } \\
\text { lamp } \\
(\lambda>420 \mathrm{~nm})\end{array}$ & $\begin{array}{c}\text { Direct } \\
\text { Z-scheme }\end{array}$ & $\begin{array}{l}\text { Increase in the } \\
\text { electron density on } \\
\text { BiOI led to internal } \\
\text { electric field } \\
\text { formation } \\
\text { favouring } \\
\text { Z-scheme } \\
\text { configuration }\end{array}$ & [113] \\
\hline Solvothermal & $\begin{array}{c}\mathrm{Bi}\left(\mathrm{NO}_{3}\right)_{3} \cdot 5 \mathrm{H}_{2} \mathrm{O}, \mathrm{KI}, \\
\mathrm{C}_{3} \mathrm{H}_{6} \mathrm{~N}_{6} \text { and } \\
\mathrm{C}_{2} \mathrm{H}_{6} \mathrm{O}_{2}\end{array}$ & $\begin{array}{c}\text { BiOI nanoplates } \\
\text { are irregularly } \\
\text { dispersed over the } \\
\text { surface of g- } \mathrm{C}_{3} \mathrm{~N}_{4} \\
\text { nanosheets }\end{array}$ & $\begin{array}{l}\text { Rhodamine B } \\
\text { (20 ppm) }\end{array}$ & $\begin{array}{c}300 \mathrm{~W} \text { Xe lamp } \\
(\lambda>420 \mathrm{~nm})\end{array}$ & $\mathrm{p}-\mathrm{n}$ & $\begin{array}{l}\text { Charge transfer } \\
\text { mode in the } \\
\mathrm{BiOI} / \mathrm{g}-\mathrm{C}_{3} \mathrm{~N}_{4} \\
\text { followed the } \\
\text { double-transfer } \\
\text { mechanism }\end{array}$ & [114] \\
\hline Solvothermal & $\begin{array}{c}\mathrm{Bi}\left(\mathrm{NO}_{3}\right)_{3} \cdot 5 \mathrm{H}_{2} \mathrm{O}, \mathrm{KI} \\
\mathrm{HNO}_{3}, \mathrm{C}_{3} \mathrm{H}_{6} \mathrm{~N}_{6} \\
\text { and } \mathrm{CH}_{4} \mathrm{~N}_{2} \mathrm{~S}\end{array}$ & $\begin{array}{c}\text { Thin nanosheets of } \\
\text { BiOI composited } \\
\text { with wrinkled } \\
\text { nanosheets of } \\
\text { g- } \mathrm{C}_{3} \mathrm{~N}_{4}\end{array}$ & $\begin{array}{c}\text { Methylene } \\
\text { blue (20 ppm) }\end{array}$ & $\begin{array}{c}50 \mathrm{~W}, 410 \mathrm{~nm} \\
\text { LED light } \\
\text { arrays }\end{array}$ & $\begin{array}{l}\text { Direct } \\
\text { Z-scheme }\end{array}$ & $\begin{array}{c}\text { Strong IEF at } \\
\text { interface occurred } \\
\text { due to difference in } \\
\text { their Fermi } \\
\text { energies was } \\
\text { proved by DFT } \\
\text { calculations }\end{array}$ & [115] \\
\hline $\begin{array}{l}\text { Ultrasonication- } \\
\text { assisted }\end{array}$ & $\begin{array}{c}\mathrm{C}_{3} \mathrm{H}_{6} \mathrm{~N}_{6} \\
\mathrm{Bi}\left(\mathrm{NO}_{3}\right)_{3} \cdot 5 \mathrm{H}_{2} \mathrm{O}, \mathrm{KI} \\
\mathrm{C}_{2} \mathrm{H}_{5} \mathrm{OH}\end{array}$ & $\begin{array}{l}\text { BiOI particles are } \\
\text { grown over the } \\
\text { surface of g- } C_{3} \mathrm{~N}_{4} \\
\text { sheets }\end{array}$ & $\begin{array}{l}\mathrm{Cr}(\mathrm{VI})(10 \\
\text { ppm) }\end{array}$ & 500 W Xe lamp & Z-scheme & $\begin{array}{l}\text { The non-radiative } \\
\text { recombination } \\
\text { process of } \\
\text { photoinduced } \\
\text { carriers at the } \\
\text { interface was } \\
\text { confirmed by pho- } \\
\text { toluminescence } \\
\text { and ESR }\end{array}$ & [116] \\
\hline \multicolumn{8}{|c|}{$\mathrm{BiOCl}-\mathrm{g}-\mathrm{C}_{3} \mathrm{~N}_{4}$} \\
\hline $\begin{array}{c}\text { Simple } \\
\text { calcination }\end{array}$ & $\begin{array}{c}\mathrm{Bi}\left(\mathrm{NO}_{3}\right)_{3} \cdot 5 \mathrm{H}_{2} \mathrm{O} \\
\mathrm{CH}_{4} \mathrm{~N}_{2} \mathrm{O}, \mathrm{C}_{6} \mathrm{H}_{14} \mathrm{O}_{6} \\
\text { and } \mathrm{NaCl}\end{array}$ & $\begin{array}{c}\mathrm{g}-\mathrm{C}_{3} \mathrm{~N}_{4} \text { nanosheets } \\
\text { acted as substrate } \\
\text { for compactly } \\
\text { anchoring } \mathrm{BiOCl} \\
\text { nanoplates }\end{array}$ & $\begin{array}{l}\text { Methyl orange } \\
\text { (10 ppm) }\end{array}$ & 300 W Xe lamp & $\begin{array}{l}\text { Binary } \\
\text { heterojunction }\end{array}$ & $\begin{array}{l}\text { Large contact } \\
\text { surface of } 2 \mathrm{D} \\
\text { hybrid structure } \\
\text { was efficient in } \\
\text { solving detrimental } \\
\text { photoinduced } \\
\text { carrier } \\
\text { recombination }\end{array}$ & [117] \\
\hline Solvothermal & $\begin{array}{c}\mathrm{Bi}\left(\mathrm{NO}_{3}\right)_{3} \cdot 5 \mathrm{H}_{2} \mathrm{O}, \\
\mathrm{NaCl}, \mathrm{PVP}, \mathrm{K}-30 \\
\mathrm{CH}_{4} \mathrm{~N}_{2} \mathrm{O} \text { and } \\
\mathrm{C}_{3} \mathrm{H}_{8} \mathrm{O}_{3}\end{array}$ & $\begin{array}{c}\text { Ultrathin } \\
\text { nanosheets of } \\
\text { BiOCl are covered } \\
\text { by } 2 \mathrm{D} \text { g- } \mathrm{C}_{3} \mathrm{~N}_{4} \\
\text { layers stacked in } \\
\text { the form of } \\
\text { multi-slice } \\
\text { structure }\end{array}$ & $\begin{array}{l}\text { 4-chlorophenol } \\
\text { (10 ppm) }\end{array}$ & $\begin{array}{c}300 \mathrm{~W} \\
\text { short-arc Xe } \\
\text { lamp }\end{array}$ & $\begin{array}{c}\text { Binary } \\
\text { heterojunction }\end{array}$ & $\begin{array}{l}\text { Introduction of } \\
\text { oxygen vacancies } \\
\text { brings a new defect } \\
\text { level for increased } \\
\text { photoabsorption }\end{array}$ & [118] \\
\hline Hydrothermal & $\begin{array}{c}\mathrm{C}_{3} \mathrm{H}_{6} \mathrm{~N}_{6}, \mathrm{NH}_{4} \mathrm{Cl} \\
\mathrm{Bi}\left(\mathrm{NO}_{3}\right)_{3} \cdot 5 \mathrm{H}_{2} \mathrm{O} \\
\text { and } \mathrm{KCl}\end{array}$ & $\begin{array}{l}\text { Smooth surface of } \\
\mathrm{BiOCl} \text { nanodiscs } \\
\text { turned rough after } \\
\text { loading ultrathin } \\
\text { g- } \mathrm{C}_{3} \mathrm{~N}_{4} \text { nanosheets }\end{array}$ & $\begin{array}{l}\text { Rhodamine B } \\
\text { (10 ppm) }\end{array}$ & 300 W Xe lamp & $\mathrm{p}-\mathrm{n}$ & $\begin{array}{l}\text { Photosensitization } \\
\text { of RhB played } \\
\text { critical role in } \\
\text { degradation } \\
\text { process over BiOCl } \\
\text { under visible light }\end{array}$ & [119] \\
\hline
\end{tabular}


Table 1. Cont

\begin{tabular}{|c|c|c|c|c|c|c|c|}
\hline $\begin{array}{l}\text { Synthesis } \\
\text { Method }\end{array}$ & Precursors & Morphology & $\begin{array}{l}\text { Contaminant } \\
\text { Parameters }\end{array}$ & Light Source & $\begin{array}{c}\text { Heterojunction } \\
\text { Type }\end{array}$ & $\begin{array}{c}\text { Significance of the } \\
\text { Result }\end{array}$ & Ref. \\
\hline $\begin{array}{l}\text { Ionic liquid- } \\
\text { assisted }\end{array}$ & $\begin{array}{c}\mathrm{C}_{2} \mathrm{H}_{4} \mathrm{~N}_{4}, \mathrm{C}_{2} \mathrm{H}_{6} \mathrm{O}_{2} \\
\mathrm{Bi}\left(\mathrm{NO}_{3}\right)_{3} \cdot 5 \mathrm{H}_{2} \mathrm{O} \\
\text { and }\left[\mathrm{C}_{16} \mathrm{mim}\right] \mathrm{Cl}\end{array}$ & $\begin{array}{c}\text { Spherical } \\
\text { microstructures } \\
\text { with large number } \\
\text { of smaller } \\
\text { nanosheets of } \\
\mathrm{BiOCl} \text { and } \mathrm{g}-\mathrm{C}_{3} \mathrm{~N}_{4}\end{array}$ & $\begin{array}{l}\text { Rhodamine B } \\
\text { (10 ppm) }\end{array}$ & 300 W Xe lamp & $\mathrm{p}-\mathrm{n}$ & $\begin{array}{c}{\left[\mathrm{C}_{16} \mathrm{mim}\right] \mathrm{Cl} \text { having }} \\
\text { positive polarity } \\
\text { improved the } \\
\text { dispersity of } \\
\text { g- } \mathrm{C}_{3} \mathrm{~N}_{4}\end{array}$ & [89] \\
\hline $\begin{array}{l}\text { In situ } \\
\text { surfactant- } \\
\text { free }\end{array}$ & $\begin{array}{c}\mathrm{C}_{3} \mathrm{H}_{6} \mathrm{~N}_{6} \\
\mathrm{Bi}\left(\mathrm{NO}_{3}\right)_{3} \cdot 5 \mathrm{H}_{2} \mathrm{O} \\
\mathrm{HCl} \text { and } \mathrm{C}_{2} \mathrm{H}_{5} \mathrm{OH}\end{array}$ & $\begin{array}{l}\text { Irregular elliptical } \\
\mathrm{BiOCl} \text { nanosheets } \\
\text { are grown over the } \\
\text { surface of } \mathrm{g}-\mathrm{C}_{3} \mathrm{~N}_{4} \\
\text { sheets }\end{array}$ & $\begin{array}{l}\text { Rhodamine B } \\
\text { (10 ppm) }\end{array}$ & 300 W Xe lamp & $\begin{array}{c}\text { Binary } \\
\text { heterojunction }\end{array}$ & $\begin{array}{l}\text { The appropriate } \\
\text { proportion of } \\
\text { BiOCl in } \\
\text { heterojunction and } \\
\text { large surface area } \\
\text { with higher } \\
\text { adsorption } \\
\text { capacity provided } \\
\text { larger photoactive } \\
\text { sites for } \\
\text { photodegradation } \\
\text { of RhB }\end{array}$ & [120] \\
\hline $\begin{array}{l}\text { Microwave- } \\
\text { assited }\end{array}$ & $\begin{array}{c}\mathrm{Bi}\left(\mathrm{NO}_{3}\right)_{3} \cdot 5 \mathrm{H}_{2} \mathrm{O} \\
\mathrm{KCl}, \mathrm{C}_{2} \mathrm{H}_{6} \mathrm{O}_{2} \\
\mathrm{C}_{2} \mathrm{H}_{4} \mathrm{~N}_{4}\end{array}$ & $\begin{array}{l}\text { Microspheres } \\
\text { assembled by } \\
\text { nanosheets }\end{array}$ & $\begin{array}{l}\text { Carbamazepine } \\
(2.5 \mathrm{ppm})\end{array}$ & $\begin{array}{l}\text { LED lamp }(\lambda> \\
\quad 420 \mathrm{~nm})\end{array}$ & $n-p$ & $\begin{array}{l}\text { Oxygen vacancies } \\
\text { can be assessed by } \\
\text { reactions using } \\
\text { ethylene glycol as a } \\
\text { solvent at a high } \\
\text { temperature }\end{array}$ & [121] \\
\hline $\begin{array}{l}\text { Microwave- } \\
\text { assisted }\end{array}$ & $\begin{array}{c}\mathrm{C}_{3} \mathrm{H}_{6} \mathrm{~N}_{6} \\
\mathrm{Bi}\left(\mathrm{NO}_{3}\right)_{3} \cdot 5 \mathrm{H}_{2} \mathrm{O} \\
\mathrm{KCl}, \mathrm{HNO}_{3}\end{array}$ & $\begin{array}{l}\mathrm{BiOCl} \text { microplates } \\
\text { were grown over } \\
\text { the surface of } \\
\mathrm{g}-\mathrm{C}_{3} \mathrm{~N}_{4} \text { nanosheets }\end{array}$ & $\begin{array}{l}\text { Nizatidine (5 } \\
\text { ppm) }\end{array}$ & Mic-LED-365 & $\begin{array}{c}\text { Binary } \\
\text { heterojunction }\end{array}$ & $\begin{array}{l}\mathrm{pH} \text { of the solution } \\
\text { was adjusted to } \\
\text { match the } \\
\text { isoelectric point of } \\
\text { the complex } \\
\text { materials for } \\
\text { enhancing the } \\
\text { photocatalytic } \\
\text { activity }\end{array}$ & [122] \\
\hline \multicolumn{8}{|c|}{$\mathrm{BiOBr}-\mathrm{g}-\mathrm{C}_{3} \mathrm{~N}_{4}$} \\
\hline $\begin{array}{l}\text { Reflux } \\
\text { process in oil } \\
\text { bath }\end{array}$ & $\begin{array}{c}\mathrm{CH}_{4} \mathrm{~N}_{2} \mathrm{O}, \mathrm{C}_{2} \mathrm{H}_{6} \mathrm{O}_{2} \\
\mathrm{Bi}\left(\mathrm{NO}_{3}\right)_{3} \cdot 5 \mathrm{H}_{2} \mathrm{O} \\
\mathrm{KBr} \text { and } \mathrm{C}_{2} \mathrm{H}_{5} \mathrm{OH}\end{array}$ & $\begin{array}{c}\mathrm{BiOBr} \text { nanoplates } \\
\text { are deposited on } \\
\text { the surface of } \\
\text { larger g- } \mathrm{C}_{3} \mathrm{~N}_{4} \\
\text { nanosheets }\end{array}$ & $\begin{array}{l}\text { Rhodamine B } \\
\text { (10 ppm) } \\
\text { Bisphenol A ( } 5 \\
\text { ppm) }\end{array}$ & 300 W Xe lamp & Z-scheme & $\begin{array}{l}\text { More reactive sites } \\
\text { and enhanced mass } \\
\text { transfer resulting } \\
\text { from larger specific } \\
\text { surface area and } \\
\text { mesoporosity led } \\
\text { to higher activity }\end{array}$ & [123] \\
\hline Hydrothermal & $\begin{array}{c}\mathrm{C}_{3} \mathrm{H}_{6} \mathrm{~N}_{6}, \mathrm{C}_{2} \mathrm{H}_{6} \mathrm{O}_{2} \\
\mathrm{Bi}\left(\mathrm{NO}_{3}\right)_{3} \cdot 5 \mathrm{H}_{2} \mathrm{O} \\
\text { and CTAB }\end{array}$ & $\begin{array}{c}\text { Nanoflakes of } \\
\mathrm{g}-\mathrm{C}_{3} \mathrm{~N}_{4} \text { and } \mathrm{BiOBr} \\
\text { are observed }\end{array}$ & $\begin{array}{l}\text { Bisphenol A } \\
\text { (10 ppm) } \\
\text { Methyl orange } \\
\text { (10 ppm) } \\
\text { Rhodamine B } \\
\text { (10 ppm) }\end{array}$ & 300 W Xe lamp & $\begin{array}{l}\text { Binary } \\
\text { heterojunction }\end{array}$ & $\begin{array}{l}\text { Surface functional } \\
\text { groups of g- } \mathrm{C}_{3} \mathrm{~N}_{4} \\
\text { provided } \\
\text { nucleation sites for } \\
\text { reaction by } \\
\text { inhibiting the } \\
\text { formation of } \mathrm{BiOBr} \\
\text { assembly }\end{array}$ & [124] \\
\hline $\begin{array}{l}\text { Electrostatic } \\
\text { self- } \\
\text { assembly }\end{array}$ & $\begin{array}{c}\mathrm{Bi}\left(\mathrm{NO}_{3}\right)_{3} \cdot 5 \mathrm{H}_{2} \mathrm{O} \\
\mathrm{KBr}, \mathrm{C}_{3} \mathrm{H}_{6} \mathrm{~N}_{6}, \mathrm{HCl}, \\
\mathrm{CH}_{3} \mathrm{COOH} \text { and } \\
\mathrm{C}_{2} \mathrm{H}_{3} \mathrm{NaO}_{2}\end{array}$ & $\begin{array}{l}\text { 3D hierarchical } \\
\text { flower-like } \\
\text { structures of } \mathrm{BiOBr} \\
\text { are attached to } \\
\text { surface of pg- } \mathrm{C}_{3} \mathrm{~N}_{4} \\
\text { consisting of } \\
\text { nanostructures and } \\
\text { plicate shapes. }\end{array}$ & $\begin{array}{l}\text { Carbamazepine } \\
\text { (5 ppm) }\end{array}$ & 500 W Xe lamp & $\begin{array}{l}\text { Binary } \\
\text { heterojunction }\end{array}$ & $\begin{array}{l}\text { Presence of low } \\
\text { concentration of } \\
\text { bicarbonate } \\
\text { accelerated the } \\
\text { carbamazepine } \\
\text { degradation while } \\
\text { nitrate and } \\
\text { chloride inhibited } \\
\text { its efficiency }\end{array}$ & {$[62]$} \\
\hline Solvothermal & $\begin{array}{l}\mathrm{Bi}\left(\mathrm{NO}_{3}\right)_{3} \cdot 5 \mathrm{H}_{2} \mathrm{O} \\
\mathrm{CTAB}, \mathrm{C}_{3} \mathrm{H}_{8} \mathrm{O}_{3} \\
\text { PVP and } \mathrm{C}_{3} \mathrm{H}_{6} \mathrm{~N}_{6}\end{array}$ & $\begin{array}{l}\text { Flower-like } \\
\text { microspheres of } \\
\mathrm{BiOBr} \text { are grown } \\
\text { over } \mathrm{g}-\mathrm{C}_{3} \mathrm{~N}_{4} \\
\text { nanosheets }\end{array}$ & $\begin{array}{l}\text { Methyl orange } \\
\text { (10 ppm) } \\
\text { Rhodamine B } \\
\quad(10 \mathrm{ppm})\end{array}$ & 500 W Xe lamp & Z-scheme & $\begin{array}{l}\text { Optimum content } \\
\text { of } g-\mathrm{C}_{3} \mathrm{~N}_{4} \text { was } \\
\text { found to be } 5 \mathrm{wt} \% \\
\text { over the } \mathrm{BiOBr} \\
\text { nanosheets }\end{array}$ & [125] \\
\hline
\end{tabular}


Table 1. Cont.

\begin{tabular}{|c|c|c|c|c|c|c|c|}
\hline $\begin{array}{l}\text { Synthesis } \\
\text { Method }\end{array}$ & Precursors & Morphology & $\begin{array}{l}\text { Contaminant } \\
\text { Parameters }\end{array}$ & Light Source & $\begin{array}{c}\text { Heterojunction } \\
\text { Type }\end{array}$ & $\begin{array}{c}\text { Significance of the } \\
\text { Result }\end{array}$ & Ref. \\
\hline $\begin{array}{l}\text { Ultrasound- } \\
\text { assisted } \\
\text { water-bath } \\
\text { deposition }\end{array}$ & $\begin{array}{c}\mathrm{Bi}\left(\mathrm{NO}_{3}\right)_{3} \cdot 5 \mathrm{H}_{2} \mathrm{O}, \\
\mathrm{PVP}, \mathrm{C}_{2} \mathrm{H}_{6} \mathrm{O}_{2} \\
\mathrm{NaBr}, \mathrm{C}_{2} \mathrm{H}_{5} \mathrm{OH} \\
\text { and } \mathrm{CH}_{4} \mathrm{~N}_{2} \mathrm{O}\end{array}$ & $\begin{array}{l}\mathrm{BiOBr} \text { nanoflakes } \\
\text { are dispersed over } \\
\text { the surface of } \\
\mathrm{g}-\mathrm{C}_{3} \mathrm{~N}_{4} \text { nanosheets }\end{array}$ & $\begin{array}{c}\text { Rhodamine B } \\
(20 \mathrm{ppm}) \\
\text { E. coli }(1.0 \times \\
\left.10^{6} \mathrm{CFU} \mathrm{mL}^{-1}\right)\end{array}$ & Visible light & Z-scheme & $\begin{array}{c}\text { Z-scheme } \\
\text { photocatalytic } \\
\text { mechanism was } \\
\text { evidenced from } \\
\text { Tafel curve analysis }\end{array}$ & [99] \\
\hline Solvothermal & $\begin{array}{c}\mathrm{Bi}\left(\mathrm{NO}_{3}\right)_{3} \cdot 5 \mathrm{H}_{2} \mathrm{O}, \\
\mathrm{C}_{3} \mathrm{H}_{6} \mathrm{~N}_{6}, \mathrm{CTAB} \\
\text { and } \mathrm{C}_{2} \mathrm{H}_{6} \mathrm{O}_{2}\end{array}$ & $\begin{array}{c}\mathrm{g}-\mathrm{C}_{3} \mathrm{~N}_{4} \text { nanosheets } \\
\text { are compactly } \\
\text { combined with } \\
\text { BiOBr nanosheets }\end{array}$ & $\begin{array}{l}\text { Rhodamine B } \\
\text { (10 ppm) }\end{array}$ & $500 \mathrm{~W}$ Xe lamp & $\begin{array}{c}\text { Binary } \\
\text { heterojunction }\end{array}$ & $\begin{array}{l}\text { Holes and } \\
\text { superoxide radicals } \\
\text { played dominant } \\
\text { role in the RhB } \\
\text { removal }\end{array}$ & [126] \\
\hline $\begin{array}{l}\text { Template- } \\
\text { assisted } \\
\text { hydrother- } \\
\text { mal }\end{array}$ & $\begin{array}{c}\mathrm{Bi}\left(\mathrm{NO}_{3}\right)_{3} \cdot 5 \mathrm{H}_{2} \mathrm{O} \\
\mathrm{NaBr}, \mathrm{C}_{2} \mathrm{H}_{6} \mathrm{O}_{2} \\
\mathrm{NaOH}, \mathrm{C}_{2} \mathrm{H}_{5} \mathrm{OH} \\
\text { and } \mathrm{NH}_{4} \mathrm{Cl}\end{array}$ & $\begin{array}{c}\mathrm{BiOBr} \\
\text { microspheres are } \\
\text { randomly } \\
\text { dispersed on the } \\
\text { surface of } \mathrm{g}-\mathrm{C}_{3} \mathrm{~N}_{4}\end{array}$ & $\begin{array}{l}\text { Tetracycline } \\
(20 \text { ppm }) \\
\text { Rhodamine B } \\
\text { (15 ppm) }\end{array}$ & $500 \mathrm{~W}$ Xe lamp & $\begin{array}{c}\text { Direct } \\
\text { Z-scheme }\end{array}$ & $\begin{array}{l}\text { L-lysine with polar } \\
\text { functional groups } \\
\text { of amino and } \\
\text { hydroxyl, served as } \\
\text { bio-template for } \\
\text { controlling the } \\
\text { crystal growth and } \\
\text { self-assembly } \\
\text { process of BiOBr }\end{array}$ & [81] \\
\hline Hydrothermal & $\begin{array}{c}\mathrm{C}_{3} \mathrm{H}_{6} \mathrm{~N}_{6} \\
\mathrm{Bi}\left(\mathrm{NO}_{3}\right)_{3} \cdot 5 \mathrm{H}_{2} \mathrm{O} \\
\mathrm{HCl}, \mathrm{NaOH} \\
\mathrm{CH}_{4} \mathrm{~N}_{2} \mathrm{O}, \mathrm{CH}_{4} \mathrm{~N}_{2} \mathrm{~S} \\
\text { and } \mathrm{KBr}\end{array}$ & $\begin{array}{l}\mathrm{BiOBr} \text { nanolayers } \\
\text { are distributed on } \\
\text { the surface of } \\
\text { porous g- } \mathrm{C}_{3} \mathrm{~N}_{4} \\
\text { nanosheets }\end{array}$ & $\begin{array}{c}\text { Methylene } \\
\text { blue (10 ppm) }\end{array}$ & $\begin{array}{l}50 \mathrm{~W} 410 \mathrm{~nm} \\
\text { LED light }\end{array}$ & $\begin{array}{c}\text { Binary } \\
\text { heterojunction }\end{array}$ & $\begin{array}{c}\text { Optimized content } \\
\text { of Pg- } \mathrm{C}_{3} \mathrm{~N}_{4} \text { in the } \\
\text { binary composite } \\
\text { for high activity } \\
\text { was found to be } 20 \\
\text { wt } \%\end{array}$ & [127] \\
\hline $\begin{array}{l}\text { Polycondensation } \\
\text { and } \\
\text { precipitation }\end{array}$ & $\begin{array}{c}\mathrm{C}_{3} \mathrm{H}_{6} \mathrm{~N}_{6} \\
\mathrm{Bi}\left(\mathrm{NO}_{3}\right)_{3} \cdot 5 \mathrm{H}_{2} \mathrm{O} \\
\text { and CTAB }\end{array}$ & $\begin{array}{c}\text { Mesoporous } \\
\text { flower-like } \mathrm{BiOBr} \\
\text { are grown over } \\
\text { porous sheets of } \\
\qquad \mathrm{g}-\mathrm{C}_{3} \mathrm{~N}_{4}\end{array}$ & $\begin{array}{c}\text { Reactive blue } \\
198 \text { (50 ppm) } \\
\text { Reactive black } \\
5 \text { (50 ppm) } \\
\text { Reactive } \\
\text { yellow } 145 \text { ( } 50 \\
\text { ppm) }\end{array}$ & $\begin{array}{c}500 \mathrm{~W} \\
\text { tungsten lamp }\end{array}$ & Z-scheme & $\begin{array}{l}\text { Degradation } \\
\text { pathways were } \\
\text { proposed to follow } \\
\text { pseudo-first-order } \\
\text { kinetics with } 30 \% \\
\text { pGCN-BiOBr }\end{array}$ & [128] \\
\hline $\begin{array}{l}\text { Reflux } \\
\text { process }\end{array}$ & $\begin{array}{c}\text { TEOS, } \mathrm{C}_{2} \mathrm{H}_{5} \mathrm{OH} \\
\mathrm{NH}_{4} \mathrm{OH}, \mathrm{C}_{2} \mathrm{H}_{4} \mathrm{~N}_{4} \\
\mathrm{NH}_{4} \mathrm{HF}_{2} \\
\mathrm{Bi}\left(\mathrm{NO}_{3}\right)_{3} \cdot 5 \mathrm{H}_{2} \mathrm{O} \\
\mathrm{CH}_{4} \mathrm{~N}_{2} \mathrm{O} \text { and } \mathrm{KBr}\end{array}$ & $\begin{array}{c}\mathrm{BiOBr} \\
\text { nanoparticles are } \\
\text { uniformly loaded } \\
\text { on the surface of IO } \\
\mathrm{CN}\end{array}$ & $\begin{array}{l}\text { Levofloxacin } \\
(10 \text { ppm }) \\
\text { Rhodamine B } \\
(20 \text { ppm })\end{array}$ & $300 \mathrm{~W}$ Xe lamp & Z-scheme & $\begin{array}{l}\text { Combination of } \\
\text { Z-scheme and } \\
\text { inverse opal } \\
\text { structure } \\
\text { influenced the } \\
\text { visible light } \\
\text { absorption ability } \\
\text { and photocatalytic } \\
\text { performance }\end{array}$ & [129] \\
\hline \multicolumn{8}{|c|}{$\mathrm{Bi}_{\mathrm{x}} \mathrm{O}_{\mathrm{y}} \mathrm{X}_{\mathrm{z}}-\mathrm{g}-\mathrm{C}_{3} \mathrm{~N}_{4}$} \\
\hline \multicolumn{8}{|c|}{$\mathrm{Bi}_{7} \mathrm{O}_{9} \mathrm{I}_{3}-\mathrm{g}-\mathrm{C}_{3} \mathrm{~N}_{4}$} \\
\hline Hydrothermal & $\begin{array}{c}\mathrm{Bi}\left(\mathrm{NO}_{3}\right)_{3} \cdot 5 \mathrm{H}_{2} \mathrm{O}, \\
\mathrm{C}_{3} \mathrm{H}_{6} \mathrm{~N}_{6}, \mathrm{NaI}, \\
\mathrm{CN}_{2} \mathrm{H}_{4} \mathrm{~S} \text { and } \\
\mathrm{CH}_{3} \mathrm{COONa}\end{array}$ & 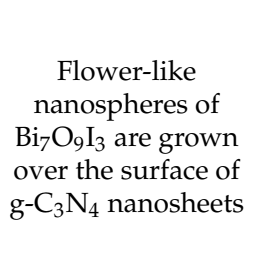 & $\begin{array}{l}\text { Doxycycline } \\
\text { hydrochloride }\end{array}$ & Xe lamp & Z-scheme & $\begin{array}{l}\text { In situ growth of } \\
\mathrm{Bi}_{7} \mathrm{O}_{9} \mathrm{I}_{3} \text { on } \\
\text { ultrathin }-\mathrm{C}_{3} \mathrm{~N}_{4} \\
\text { via mild and } \\
\text { simple } \\
\text { hydrothermal } \\
\text { means without any } \\
\text { toxic reagents }\end{array}$ & [109] \\
\hline \multicolumn{8}{|c|}{$\mathrm{Bi}_{5} \mathrm{O}_{7} \mathrm{I}-\mathrm{g}-\mathrm{C}_{3} \mathrm{~N}_{4}$} \\
\hline Hydrothermal & $\begin{array}{c}\mathrm{NH}_{3}, \mathrm{C}_{2} \mathrm{H}_{5} \mathrm{OH}, \\
\text { TEOS, C18TMOS, } \\
\mathrm{CH}_{2} \mathrm{~N}_{2}, \mathrm{NH}_{4} \mathrm{HF}_{2}, \\
\mathrm{Bi}\left(\mathrm{NO}_{3}\right)_{3} \cdot 5 \mathrm{H}_{2} \mathrm{O} \\
\mathrm{C}_{2} \mathrm{H}_{6} \mathrm{O}_{2} \text { and } \mathrm{KI}\end{array}$ & $\begin{array}{c}\mathrm{Bi}_{5} \mathrm{O}_{7} \mathrm{I} \\
\text { nanoparticles are } \\
\text { grown over surface } \\
\text { of porous } \mathrm{g}-\mathrm{C}_{3} \mathrm{~N}_{4}\end{array}$ & $\begin{array}{l}\text { Phenol (10 } \\
\text { ppm) }\end{array}$ & CEL-HXF300 & p-n & $\begin{array}{l}\text { Silica templates } \\
\text { were used to obtain } \\
\text { lamellar and } \\
\text { porous } \mathrm{g}-\mathrm{C}_{3} \mathrm{~N}_{4} \text { in } \\
\text { GCN-Bis } \mathrm{O}_{7} \mathrm{I} \\
\text { composite }\end{array}$ & [130] \\
\hline
\end{tabular}


Table 1. Cont.

\begin{tabular}{|c|c|c|c|c|c|c|c|}
\hline $\begin{array}{l}\text { Synthesis } \\
\text { Method }\end{array}$ & Precursors & Morphology & $\begin{array}{l}\text { Contaminant } \\
\text { Parameters }\end{array}$ & Light Source & $\begin{array}{l}\text { Heterojunction } \\
\text { Type }\end{array}$ & $\begin{array}{c}\text { Significance of the } \\
\text { Result }\end{array}$ & Ref. \\
\hline Hydrothermal & $\begin{array}{c}\mathrm{Bi}\left(\mathrm{NO}_{3}\right)_{3} \cdot 5 \mathrm{H}_{2} \mathrm{O}, \mathrm{KI} \\
\mathrm{C}_{2} \mathrm{H}_{6} \mathrm{O}_{2} \text { and } \\
\mathrm{C}_{3} \mathrm{H}_{6} \mathrm{~N}_{6}\end{array}$ & $\begin{array}{l}\text { Irregular shaped } \\
\text { layers of g- } \mathrm{C}_{3} \mathrm{~N}_{4} \\
\text { are covered on the } \\
\text { surface of } \\
\text { microspheres } \\
\text { consisting of } \\
\text { self-assembled thin } \\
\text { platelets of } \mathrm{Bi}_{5} \mathrm{O}_{7} \mathrm{I}\end{array}$ & $\begin{array}{l}\text { Methyl orange } \\
\text { (10 ppm) } \\
\text { Rhodamine B } \\
\text { (10 ppm) }\end{array}$ & $300 \mathrm{~W}$ Xe lamp & $\begin{array}{c}\text { Z-scheme } \\
\text { Heterojunction }\end{array}$ & $\begin{array}{c}\text { g- } \mathrm{C}_{3} \mathrm{~N}_{4}-\mathrm{Bi}_{5} \mathrm{O}_{7} \mathrm{I}-10 \\
\text { showed better } \\
\text { performance } \\
\text { towards dye } \\
\text { degradation in } \\
\text { acidic conditions }\end{array}$ & [107] \\
\hline $\begin{array}{l}\text { Hydrolysis } \\
\text { and thermal } \\
\text { condensa- } \\
\text { tion }\end{array}$ & $\begin{array}{c}\mathrm{C}_{2} \mathrm{H}_{4} \mathrm{~N}_{4} \\
\mathrm{Bi}\left(\mathrm{NO}_{3}\right)_{3} \cdot 4 \mathrm{H}_{2} \mathrm{O}, \\
\mathrm{C}_{2} \mathrm{H}_{6} \mathrm{O}_{2}, \mathrm{NaOH}, \\
\mathrm{HCl}_{6} \mathrm{C}_{6} \mathrm{H}_{15} \mathrm{~N}, \\
\mathrm{KH}_{2} \mathrm{PO}_{4} \text { and } \mathrm{KI}\end{array}$ & $\begin{array}{c}\text { Rod-like patterns } \\
\text { of } \mathrm{Bi}_{5} \mathrm{O}_{7} \mathrm{I} \text { are } \\
\text { embedded on } \\
\mathrm{D}-\mathrm{g}-\mathrm{C}_{3} \mathrm{~N}_{4}\end{array}$ & $\begin{array}{l}\text { Metronidazole } \\
\quad(15 \mathrm{ppm})\end{array}$ & $300 \mathrm{~W}$ Xe lamp & $\begin{array}{c}\text { Binary } \\
\text { heterojunction }\end{array}$ & $\begin{array}{l}\text { Charge carrier } \\
\text { separation in the } \\
\text { composite was } \\
\text { evidenced from } \\
\text { photocurrent } \\
\text { response } \\
\text { measurements }\end{array}$ & [131] \\
\hline \multicolumn{8}{|c|}{$\mathrm{Bi}_{4} \mathrm{O}_{5} \mathrm{I}_{2}-\mathrm{g}-\mathrm{C}_{3} \mathrm{~N}_{4}$} \\
\hline $\begin{array}{l}\text { Mixed } \\
\text { calcination }\end{array}$ & $\begin{array}{c}\mathrm{C}_{3} \mathrm{H}_{6} \mathrm{~N}_{6} \\
\mathrm{Bi}\left(\mathrm{NO}_{3}\right)_{3} \cdot 4 \mathrm{H}_{2} \mathrm{O}, \\
\mathrm{NaOH} \text { and } \mathrm{KI}\end{array}$ & $\begin{array}{l}\mathrm{Bi}_{4} \mathrm{O}_{5} \mathrm{I}_{2} \text { nanoflakes } \\
\text { are grown on } \\
\text { g- } \mathrm{C}_{3} \mathrm{~N}_{4} \text { nanosheets }\end{array}$ & $\begin{array}{l}\text { Rhodamine B } \\
\left(1 \times 10^{-5} \mathrm{M}\right) \\
\text { NO removal }\end{array}$ & $\begin{array}{c}300 \mathrm{~W} \\
\text { tungsten } \\
\text { halogen lamp }\end{array}$ & $\mathrm{n}-\mathrm{n}$ & $\begin{array}{c}\text { Super oxide } \\
\text { radicals and holes } \\
\text { are active species } \\
\text { during the } \\
\text { degradation }\end{array}$ & [132] \\
\hline $\begin{array}{l}\text { Ionic liquid- } \\
\text { assisted } \\
\text { solvother- } \\
\text { mal }\end{array}$ & $\begin{array}{c}\mathrm{C}_{6} \mathrm{H}_{14} \mathrm{O}_{6}, \\
\mathrm{C}_{10} \mathrm{H}_{19} \mathrm{IN}_{2} \\
\mathrm{Bi}\left(\mathrm{NO}_{3}\right)_{3} \cdot 4 \mathrm{H}_{2} \mathrm{O} \\
\mathrm{NaOH} \text { and } \mathrm{KI}\end{array}$ & $\begin{array}{l}\mathrm{Bi}_{4} \mathrm{O}_{5} \mathrm{I}_{2} \text { nanosheets } \\
\text { are dispersed on } \\
\text { g- } \mathrm{C}_{3} \mathrm{~N}_{4} \text { nanosheets }\end{array}$ & $\begin{array}{l}\text { Rhodamine B } \\
\text { (10 ppm) } \\
\text { Bisphenol A } \\
(10 \text { ppm })\end{array}$ & $300 \mathrm{~W}$ Xe lamp & $\begin{array}{c}\text { Binary } \\
\text { hetrojunction }\end{array}$ & $\begin{array}{l}\text { [Hmim]I played } \\
\text { multiple roles } \\
\text { during the } \\
\text { synthesis which } \\
\text { was propitious for } \\
\text { heterojunction } \\
\text { formation }\end{array}$ & [87] \\
\hline $\begin{array}{l}\text { Hydrothermal } \\
\text { and heating }\end{array}$ & $\begin{array}{c}\mathrm{C}_{3} \mathrm{H}_{6} \mathrm{~N}_{6} \\
\mathrm{Bi}\left(\mathrm{NO}_{3}\right)_{3} \cdot 4 \mathrm{H}_{2} \mathrm{O}, \\
\mathrm{C}_{2} \mathrm{H}_{6} \mathrm{O}_{2} \text { and } \mathrm{KI}\end{array}$ & $\begin{array}{c}\text { Hierarchical } \\
\text { microspheres of } \\
\mathrm{Bi}_{4} \mathrm{O}_{5} \mathrm{I}_{2} \text { are grown } \\
\text { on the surface of } \\
{\mathrm{g}-\mathrm{C}_{3} \mathrm{~N}_{4}}\end{array}$ & $\begin{array}{l}\text { Methyl orange } \\
\text { (20 ppm) }\end{array}$ & $350 \mathrm{~W}$ Xe lamp & Type-II & $\begin{array}{l}\text { The in situ } \\
\text { transformation } \\
\text { endowed the } \\
\text { composite with } \\
\text { good contact } \\
\text { between the } \\
\text { semiconductors in } \\
\text { construction of } \\
\text { tight } \\
\text { heterojunction }\end{array}$ & [110] \\
\hline \multicolumn{8}{|c|}{$\mathrm{Bi}_{3} \mathrm{O}_{4} \mathrm{Cl}-\mathrm{g}-\mathrm{C}_{3} \mathrm{~N}_{4}$} \\
\hline $\begin{array}{l}\text { Mixing and } \\
\text { heating }\end{array}$ & $\begin{array}{c}\mathrm{Bi}\left(\mathrm{NO}_{3}\right)_{3} \cdot 5 \mathrm{H}_{2} \mathrm{O} \\
\mathrm{HCl}, \mathrm{Bi}_{2} \mathrm{O}_{3} \text { and } \\
\quad \mathrm{C}_{3} \mathrm{H}_{6} \mathrm{~N}_{6}\end{array}$ & $\begin{array}{c}\text { Irregular blocks } \\
\text { consisting of a lot } \\
\text { of nanoflakes of } \\
\mathrm{Bi}_{3} \mathrm{O}_{4} \mathrm{Cl} \text { attached } \\
\text { onto the surface of } \\
\quad \mathrm{g}-\mathrm{C}_{3} \mathrm{~N}_{4}\end{array}$ & $\begin{array}{l}\text { Rhodamine B } \\
\text { (10 ppm) }\end{array}$ & $350 \mathrm{~W}$ Xe lamp & $\begin{array}{c}\text { Binary } \\
\text { heterojunction }\end{array}$ & $\begin{array}{c}\text { Coupling } \mathrm{Bi}_{3} \mathrm{O}_{4} \mathrm{Cl} \\
\text { on g- } \mathrm{C}_{3} \mathrm{~N}_{4} \\
\text { improved the } \\
\text { specific surface } \\
\text { area and charge } \\
\text { carrier separation }\end{array}$ & [133] \\
\hline $\begin{array}{l}\text { Solid phase } \\
\text { calcination }\end{array}$ & $\begin{array}{c}\mathrm{Bi}\left(\mathrm{NO}_{3}\right)_{3} \cdot 5 \mathrm{H}_{2} \mathrm{O} \\
\mathrm{CH}_{4} \mathrm{~N}_{2} \mathrm{O}, \mathrm{NH}_{4} \mathrm{Cl} \\
\text { and } \mathrm{C}_{2} \mathrm{H}_{6} \mathrm{O}_{2}\end{array}$ & $\begin{array}{l}\mathrm{Bi}_{3} \mathrm{O}_{4} \mathrm{Cl} \text { nanoflakes } \\
\text { are grown on } \\
\text { g- } \mathrm{C}_{3} \mathrm{~N}_{4} \text { nanosheets }\end{array}$ & $\begin{array}{l}\text { Rhodamine B } \\
\text { (10 ppm) } \\
\text { Tetracycline } \\
\text { (10 ppm) } \\
\text { Hexavalent } \\
\text { chromium (10 } \\
\text { ppm) }\end{array}$ & $250 \mathrm{~W}$ Xe lamp & Z-scheme & $\begin{array}{l}\text { Shorter fluorescent } \\
\text { lifetime (0.952 ns) } \\
\text { attributed to } \\
\text { additional } \\
\text { nonradioactive } \\
\text { decay channel for } \\
\text { electron transfer } \\
\text { from } \mathrm{Bi}_{3} \mathrm{O}_{4} \mathrm{Cl} \text { to } \\
{\mathrm{g}-\mathrm{C}_{3} \mathrm{~N}_{4}}\end{array}$ & [96] \\
\hline \multicolumn{8}{|c|}{$\mathrm{Bi}_{12} \mathrm{O}_{17} \mathrm{Cl}_{2}-\mathrm{g}-\mathrm{C}_{3} \mathrm{~N}_{4}$} \\
\hline $\begin{array}{l}\text { Chemical } \\
\text { precipitation }\end{array}$ & $\begin{array}{c}\mathrm{CH}_{4} \mathrm{~N}_{2} \mathrm{O}, \mathrm{BiCl}_{3}, \\
\mathrm{C}_{2} \mathrm{H}_{5} \mathrm{OH} \text { and } \\
\mathrm{NaOH}\end{array}$ & $\begin{array}{c}\mathrm{Bi}_{12} \mathrm{O}_{17} \mathrm{Cl}_{2} \\
\text { nanosheets are } \\
\text { grown on surface } \\
\text { of } \mathrm{g}-\mathrm{C}_{3} \mathrm{~N}_{4}\end{array}$ & $\begin{array}{l}\text { Rhodamine B } \\
\quad(5 \text { ppm }) \\
\text { Methyl orange } \\
(10 \text { ppm })\end{array}$ & 300 W Xe lamp & $\begin{array}{c}\text { Binary } \\
\text { heterojunction }\end{array}$ & $\begin{array}{l}\text { Hydroxyl radicals } \\
\text { and holes main } \\
\text { active species } \\
\text { during the reaction } \\
\text { as evidenced from } \\
\text { electron spin } \\
\text { resonance } \\
\text { technique }\end{array}$ & [60] \\
\hline
\end{tabular}


Table 1. Cont.

\begin{tabular}{|c|c|c|c|c|c|c|c|}
\hline $\begin{array}{l}\text { Synthesis } \\
\text { Method }\end{array}$ & Precursors & Morphology & $\begin{array}{c}\text { Contaminant } \\
\text { Parameters }\end{array}$ & Light Source & $\begin{array}{l}\text { Heterojunction } \\
\text { Type }\end{array}$ & $\begin{array}{c}\text { Significance of the } \\
\text { Result }\end{array}$ & Ref. \\
\hline \multicolumn{8}{|c|}{$\mathrm{Bi}_{4} \mathrm{O}_{5} \mathrm{Br}_{2}-\mathrm{g}-\mathrm{C}_{3} \mathrm{~N}_{4}$} \\
\hline $\begin{array}{l}\text { Ionic liquid- } \\
\text { assisted } \\
\text { solvother- } \\
\text { mal }\end{array}$ & $\begin{array}{c}{\left[\mathrm{C}_{16} \operatorname{mim}\right] \mathrm{Br},} \\
\mathrm{C}_{3} \mathrm{~N}_{3}\left(\mathrm{NH}_{2}\right)_{3} \\
\mathrm{C}_{2} \mathrm{H}_{3} \mathrm{~N}, \mathrm{C}_{3} \mathrm{~N}_{3} \mathrm{Cl}_{3} \\
\mathrm{Bi}\left(\mathrm{NO}_{3}\right)_{3} \cdot 5 \mathrm{H}_{2} \mathrm{O} \\
\mathrm{C}_{6} \mathrm{H}_{14} \mathrm{O}_{6} \text { and } \\
\mathrm{NaOH}\end{array}$ & $\begin{array}{c}\text { Rod-like g- } \mathrm{C}_{3} \mathrm{~N}_{4} \\
\text { has closely } \\
\text { combined with } \\
\text { sheet-like } \mathrm{Bi}_{4} \mathrm{O}_{5} \mathrm{Br}_{2}\end{array}$ & $\begin{array}{l}\text { Ciprofloxacin } \\
\text { (10 ppm) } \\
\text { Rhodamine B } \\
(10 \text { ppm })\end{array}$ & 300 W Xe lamp & $\begin{array}{c}\text { Binary } \\
\text { heterojunction }\end{array}$ & $\begin{array}{c}\text { Ionic liquid } \\
{\left[\mathrm{C}_{16} \text { mim]Br served }\right.} \\
\text { as solvent, } \\
\text { dispersing agent } \\
\text { and reactant for the } \\
\text { distribution of } \\
\mathrm{Bi}_{4} \mathrm{O}_{5} \mathrm{Br}_{2} \text { over } \\
{\mathrm{g}-\mathrm{C}_{3} \mathrm{~N}_{4}}\end{array}$ & [134] \\
\hline Precipitation & $\begin{array}{c}\mathrm{C}_{3} \mathrm{H}_{6} \mathrm{~N}_{6}, \mathrm{C}_{2} \mathrm{H}_{5} \mathrm{OH}, \\
\mathrm{BiBr}_{3} \text { and } \mathrm{NaOH}\end{array}$ & $\begin{array}{c}\text { Irregular } \\
\text { nanosheets of } \\
\mathrm{Bi}_{4} \mathrm{O}_{5} \mathrm{Br}_{2} \text { were } \\
\text { stacked with } \\
\text { g- } \mathrm{C}_{3} \mathrm{~N}_{4} \text { sheets }\end{array}$ & $\begin{array}{l}\text { Rhodamine B } \\
\text { (10 ppm) } \\
\text { Tetracycline } \\
\text { (10 ppm) }\end{array}$ & $\begin{array}{l}72 \text { W LED } \\
\text { lamp }\end{array}$ & $\begin{array}{c}\text { Binary } \\
\text { heterojunction }\end{array}$ & $\begin{array}{c}\text { Improved } \\
\text { adsorptive nature } \\
\text { in } \mathrm{BBO} / \mathrm{CN}-75 \text { is } \\
\text { due to generation } \\
\text { of more Lewis base } \\
\text { sites as confirmed } \\
\text { by } \\
\text { Zeta potential } \\
\text { studies }\end{array}$ & [135] \\
\hline Solvothermal & $\begin{array}{c}\mathrm{C}_{3} \mathrm{H}_{6} \mathrm{~N}_{6}, \\
\mathrm{Bi}\left(\mathrm{NO}_{3}\right)_{3} \cdot 5 \mathrm{H}_{2} \mathrm{O} \\
{\left[\mathrm{C}_{16} \mathrm{mim}\right] \mathrm{Br}} \\
\mathrm{C}_{6} \mathrm{H}_{14} \mathrm{O}_{6}, \mathrm{NaOH} \\
\text { and } \mathrm{C}_{2} \mathrm{H}_{5} \mathrm{OH}\end{array}$ & $\begin{array}{l}\text { Ultrathin } \mathrm{Bi}_{4} \mathrm{O}_{5} \mathrm{Br}_{2} \\
\text { nanosheets are } \\
\text { dispersed on the } \\
\text { graphene-like } \\
\text { g- } \mathrm{C}_{3} \mathrm{~N}_{4} \text { nanosheets }\end{array}$ & $\begin{array}{l}\text { Ciprofloxacin } \\
\text { (10 ppm) } \\
\text { Rhodamine B } \\
(10 \text { ppm })\end{array}$ & $300 \mathrm{~W}$ Xe lamp & $\begin{array}{c}\text { Binary } \\
\text { heterojunction }\end{array}$ & $\begin{array}{l}\text { Red shift in the } \\
\text { bandgap } \\
\text { absorption was } \\
\text { observed with } \\
\text { introduction of } \\
\text { graphene-like } \\
\text { g- } \mathrm{C}_{3} \mathrm{~N}_{4} \\
\end{array}$ & [85] \\
\hline \multicolumn{8}{|c|}{ Noble metal coupled BiOX-g- $\mathrm{C}_{3} \mathrm{~N}_{4}$} \\
\hline \multicolumn{8}{|c|}{$\mathrm{BiOI} / \mathrm{Pt} / \mathrm{g}-\mathrm{C}_{3} \mathrm{~N}_{4}$} \\
\hline $\begin{array}{l}\text { Two-step } \\
\text { (reduction } \\
\text { and stirring) }\end{array}$ & $\begin{array}{c}\mathrm{CH}_{4} \mathrm{~N}_{2} \mathrm{O}, \mathrm{NaBH}_{4} \\
\mathrm{H}_{2} \mathrm{PtCl}_{6} \cdot 6 \mathrm{H}_{2} \mathrm{O} \\
\mathrm{C}_{2} \mathrm{H}_{6} \mathrm{O}_{2} \\
\mathrm{Bi}\left(\mathrm{NO}_{3}\right)_{3} \cdot 5 \mathrm{H}_{2} \mathrm{O} \\
\text { and } \mathrm{KI}\end{array}$ & $\begin{array}{c}\text { Pt nanoparticles } \\
\text { and } \mathrm{BiOI} \\
\text { hierarchical } \\
\text { structure grew on } \\
\text { the } \mathrm{g}-\mathrm{C}_{3} \mathrm{~N}_{4} \text { sheets }\end{array}$ & $\begin{array}{l}\text { Phenol (25 } \\
\text { ppm) } \\
\text { Tetracycline } \\
\text { hydrochloride } \\
\text { (20 ppm) }\end{array}$ & Visible light & $\begin{array}{l}\text { Solid-state } \\
\text { Z-scheme }\end{array}$ & $\begin{array}{l}\text { Unobstructed } \\
\mathrm{Z} \text {-scheme charge } \\
\text { carrier transfer } \\
\text { pathways in } \\
\mathrm{BiOI} / \mathrm{Pt} / \mathrm{g}-\mathrm{C}_{3} \mathrm{~N}_{4} \\
\text { composite are } \\
\text { discussed in } \\
\text { relevance to phenol } \\
\text { and tetracycline } \\
\text { oxidation }\end{array}$ & [136] \\
\hline \multicolumn{8}{|c|}{$\mathrm{g}-\mathrm{C}_{3} \mathrm{~N}_{4} / \mathrm{Eu} / \mathrm{Bi}_{24} \mathrm{O}_{31} \mathrm{Cl}_{10}$ (BOC) } \\
\hline $\begin{array}{l}\text { Impregnation- } \\
\text { calcination }\end{array}$ & $\begin{array}{c}\mathrm{Bi}\left(\mathrm{NO}_{3}\right)_{3} \cdot 5 \mathrm{H}_{2} \mathrm{O}, \\
\mathrm{C}_{3} \mathrm{H}_{6} \mathrm{~N}_{6}, \mathrm{NH}_{4} \mathrm{Cl}, \\
\mathrm{C}_{6} \mathrm{H}_{8} \mathrm{O}_{7}, \mathrm{HNO}_{3}, \\
\mathrm{NH}_{3} \cdot \mathrm{H}_{2} \mathrm{O} \text { and } \\
\mathrm{Eu}\left(\mathrm{NO}_{3}\right)_{3} \cdot 6 \mathrm{H}_{2} \mathrm{O}\end{array}$ & $\begin{array}{l}\mathrm{g}-\mathrm{C}_{3} \mathrm{~N}_{4} \text { nanosheets } \\
\text { were coated on the } \\
\text { surface of irregular } \\
\text { shaped smaller } \\
\text { sized crystal } \\
\text { particles of } \\
\text { Eu-doped BOC }\end{array}$ & $\begin{array}{l}\text { Rhodamine B } \\
\text { (10 ppm) }\end{array}$ & 250 W Xe lamp & $\begin{array}{c}\text { Binary } \\
\text { heterojunction }\end{array}$ & $\begin{array}{c}\mathrm{CN} / \mathrm{Eu}-\mathrm{BOC} \\
\text { exhibited higher } \\
\text { performance than } \\
\mathrm{CN} / \mathrm{BOC} \\
\text { suggesting that } \\
\text { Eu (III) could be } \\
\text { used as cocatalyst }\end{array}$ & [137] \\
\hline \multicolumn{8}{|c|}{$\mathrm{g}-\mathrm{C}_{3} \mathrm{~N}_{4} / \mathrm{Au} / \mathrm{BiOBr}$} \\
\hline $\begin{array}{l}\text { Hydrothermal } \\
\text { and in situ } \\
\text { reduction }\end{array}$ & $\begin{array}{c}\mathrm{C}_{3} \mathrm{H}_{6} \mathrm{~N}_{6} \\
\mathrm{Bi}\left(\mathrm{NO}_{3}\right)_{3} \cdot 5 \mathrm{H}_{2} \mathrm{O}, \\
\mathrm{KBr}, \mathrm{C}_{2} \mathrm{H}_{5} \mathrm{OH} \\
\mathrm{C}_{8} \mathrm{H}_{11} \mathrm{NO}_{2} \text { and } \\
\mathrm{HAuCl}_{4} \cdot 4 \mathrm{H}_{2} \mathrm{O}\end{array}$ & $\begin{array}{l}\text { Au nanoparticles } \\
\text { are decorated over } \\
\text { the surface of } \\
\text { lamellar structure } \\
\text { of } \mathrm{g}-\mathrm{C}_{3} \mathrm{~N}_{4} \text { and } \\
\mathrm{BiOBr} \text { sheets }\end{array}$ & $\begin{array}{l}\text { Phenol (10 } \\
\text { ppm) }\end{array}$ & $300 \mathrm{~W}$ Xe lamp & $\begin{array}{l}\text { Plasmonic } \\
\text { Z-scheme }\end{array}$ & $\begin{array}{l}\text { Strong surface } \\
\text { plasmon resonance } \\
\text { caused by Au NPs } \\
\text { contributed to } \\
\text { extension of visible } \\
\text { light absorption in } \\
\text { the ternary } \\
\text { composite }\end{array}$ & [138] \\
\hline $\begin{array}{l}\text { Chemical } \\
\text { reduction }\end{array}$ & $\begin{array}{c}\mathrm{Bi}\left(\mathrm{NO}_{3}\right)_{3} \cdot 5 \mathrm{H}_{2} \mathrm{O} \\
\mathrm{KBr}, \mathrm{CH}_{4} \mathrm{~N}_{2} \mathrm{~S} \\
\mathrm{CTAB}, \mathrm{Na}_{3} \mathrm{C}_{6} \mathrm{H}_{5} \mathrm{O}_{7} \\
\mathrm{AuCl}_{3} \text { and } \\
\mathrm{C}_{2} \mathrm{H}_{5} \mathrm{OH}\end{array}$ & $\begin{array}{l}\text { Au nanoparticles } \\
\text { were uniformly } \\
\text { distributed over } \\
\text { the surface of } \\
\mathrm{g}-\mathrm{C}_{3} \mathrm{~N}_{4} / \mathrm{BiOBr}\end{array}$ & $\begin{array}{l}\text { Rhodamine } \mathrm{B} \\
(10 \mathrm{ppm}) \\
\mathrm{CO}_{2} \text { reduction }\end{array}$ & $300 \mathrm{~W}$ Xe lamp & $\begin{array}{l}\text { Surface } \\
\text { plasmon } \\
\text { resonance and } \\
\text { Z-scheme }\end{array}$ & $\begin{array}{c}\text { Correlation } \\
\text { between size of } \mathrm{Au} \\
\text { NPs and } \\
\text { wavelength } \\
\text { dependent } \\
\text { photocatalytic } \\
\text { activity associated } \\
\text { with } \\
\text { Au-GCN-BiOBr } \\
\text { composite is } \\
\text { described }\end{array}$ & [139] \\
\hline
\end{tabular}


Table 1. Cont.

\begin{tabular}{|c|c|c|c|c|c|c|c|}
\hline $\begin{array}{l}\text { Synthesis } \\
\text { Method }\end{array}$ & Precursors & Morphology & $\begin{array}{l}\text { Contaminant } \\
\text { Parameters }\end{array}$ & Light Source & $\begin{array}{l}\text { Heterojunction } \\
\text { Type }\end{array}$ & $\begin{array}{c}\text { Significance of the } \\
\text { Result }\end{array}$ & Ref. \\
\hline \multicolumn{8}{|c|}{ Carbon material coupled BiOX-g- $\mathrm{C}_{3} \mathrm{~N}_{4}$} \\
\hline \multicolumn{8}{|c|}{$\mathrm{g}-\mathrm{C}_{3} \mathrm{~N}_{4} / \mathrm{CDs} / \mathrm{BiOI}$} \\
\hline Precipitation & $\begin{array}{c}\mathrm{CN}_{2} \mathrm{H}_{2}, \mathrm{C}_{3} \mathrm{H}_{6} \mathrm{~N}_{6} \\
\mathrm{C}_{6} \mathrm{H}_{8} \mathrm{O}_{7} \\
\mathrm{Bi}\left(\mathrm{NO}_{3}\right)_{3} \cdot 4 \mathrm{H}_{2} \mathrm{O} \\
\text { and } \mathrm{NaI}\end{array}$ & $\begin{array}{c}\text { CDs and BiOI } \\
\text { nanoparticles are } \\
\text { grown in intimate } \\
\text { contact with gCN } \\
\text { nanosheets }\end{array}$ & $\begin{array}{l}\text { Rhodamine B } \\
\left(2.5 \times 10^{-5} \mathrm{M}\right) \\
\text { Methylene } \\
\text { blue }(2.5 \times \\
\left.10^{-5} \mathrm{M}\right) \\
\text { Methyl orange } \\
\left(2.5 \times 10^{-5} \mathrm{M}\right) \\
\text { Fuchsine }(9.20 \\
\left.\times 10^{-6} \mathrm{M}\right)\end{array}$ & $\begin{array}{l}50 \text { W LED } \\
\text { lamp }\end{array}$ & $\begin{array}{c}\text { Ternary } \\
\text { heterojunction }\end{array}$ & $\begin{array}{l}\text { Co-operative } \\
\text { effects of CQDs } \\
\text { and g- } \mathrm{C}_{3} \mathrm{~N}_{4} \\
\text { promoted the } \\
\text { activity of BiOI } \\
\text { towards the } \\
\text { degradation of } \\
\text { organic dyes }\end{array}$ & [140] \\
\hline \multicolumn{8}{|c|}{$\mathrm{GO} / \mathrm{g}-\mathrm{C}_{3} \mathrm{~N}_{4} / \mathrm{BiOI}$} \\
\hline $\begin{array}{l}\text { In situ } \\
\text { generation }\end{array}$ & $\begin{array}{c}\mathrm{CH}_{4} \mathrm{~N}_{2} \mathrm{O} \\
\mathrm{Bi}\left(\mathrm{NO}_{3}\right)_{3} \cdot 4 \mathrm{H}_{2} \mathrm{O} \\
\mathrm{C}_{2} \mathrm{H}_{6} \mathrm{O}_{2}, \mathrm{KI} \text { and } \\
\mathrm{GO}\end{array}$ & $\begin{array}{c}\text { Flower-like BiOI } \\
\text { nanosheets are } \\
\text { overlapped with } \\
\text { lamellar structure } \\
\text { of CN and } \\
\text { sheet-like GO. }\end{array}$ & $\begin{array}{l}\text { Methyl orange } \\
(10 \text { ppm }) \\
\text { Tetracycline } \\
(20 \mathrm{ppm}) \\
\text { E. coli (50 ppm) } \\
\text { S. aureus (50 } \\
\text { ppm) }\end{array}$ & LED lamp & $\begin{array}{c}\text { Ternary } \\
\text { heterojunction }\end{array}$ & $\begin{array}{l}\text { Loading GO over } \\
\mathrm{CN} / \mathrm{BiOI} \text { resulted } \\
\text { in double-charge- } \\
\text { transfer at the } \\
\text { interface }\end{array}$ & [141] \\
\hline \multicolumn{8}{|c|}{$\mathrm{g}-\mathrm{C}_{3} \mathrm{~N}_{4} / \mathrm{MCNTs} / \mathrm{BiOI}$} \\
\hline Solvothermal & $\begin{array}{c}\mathrm{CN}_{2} \mathrm{H}_{2}, \mathrm{KI}, \\
\mathrm{Bi}\left(\mathrm{NO}_{3}\right)_{3} \cdot 4 \mathrm{H}_{2} \mathrm{O} \\
\text { and } \mathrm{C}_{2} \mathrm{H}_{6} \mathrm{O}_{2}\end{array}$ & $\begin{array}{l}\text { BiOI nanoparticles } \\
\text { are uniformly } \\
\text { loaded on surface } \\
\text { of } g-C_{3} \mathrm{~N}_{4}-\mathrm{MCNTs} \text {. }\end{array}$ & $\begin{array}{c}\text { Methylene } \\
\text { blue (10 ppm) }\end{array}$ & $300 \mathrm{~W}$ Xe lamp & Z-scheme & $\begin{array}{l}\text { MCNTs facilitated } \\
\text { the electron } \\
\text { transfer from BiOI } \\
\text { to g- } \mathrm{C}_{3} \mathrm{~N}_{4} \text { resulting } \\
\text { in } \mathrm{Z} \text {-scheme charge } \\
\text { transfer pathway }\end{array}$ & [142] \\
\hline \multicolumn{8}{|c|}{$\mathrm{g}-\mathrm{C}_{3} \mathrm{~N}_{4} / \mathrm{BiOI} / \mathrm{rGO}$ immobilized on $\mathrm{Ni}$ foam } \\
\hline $\begin{array}{l}\text { Hydrothermal } \\
\text { and } \\
\text { reduction }\end{array}$ & $\begin{array}{c}\mathrm{CH}_{4} \mathrm{~N}_{2} \mathrm{O}, \mathrm{C}_{2} \mathrm{H}_{4} \mathrm{~N}_{4} \\
\mathrm{NaSO}_{4} \\
\mathrm{Bi}\left(\mathrm{NO}_{3}\right)_{3} \cdot 5 \mathrm{H}_{2} \mathrm{O} \\
\mathrm{C}_{2} \mathrm{H}_{6} \mathrm{O}_{2}, \mathrm{C}_{2} \mathrm{H}_{6} \mathrm{O} \\
\mathrm{NH}_{3}, \mathrm{H}_{4} \mathrm{~N}_{2} \cdot \mathrm{H}_{2} \mathrm{O} \\
\mathrm{Ni} \text { foam, GO and } \\
\mathrm{NaI}\end{array}$ & $\begin{array}{l}\text { Laminar structures } \\
\text { of g- } \mathrm{C}_{3} \mathrm{~N}_{4}, \mathrm{BiOI} \\
\text { and sheet-like rGO } \\
\text { form the ternary } \\
\text { sheet-like hybrids } \\
\text { and are } \\
\text { immobilized on the } \\
\text { surface of Ni foam. }\end{array}$ & $\begin{array}{l}\text { Methyl orange } \\
\qquad(5 \mathrm{ppm}) \\
\mathrm{CO}_{2}\end{array}$ & $300 \mathrm{~W}$ Xe lamp & $\begin{array}{l}\text { Hybrid } \\
\text { Z-scheme }\end{array}$ & $\begin{array}{l}\text { rGO functioned as } \\
\text { both electron } \\
\text { mediator and } \\
\text { binder while } \mathrm{Ni} \\
\text { foam improved the } \\
\text { reusability of the } \\
\text { composite }\end{array}$ & [143] \\
\hline \multicolumn{8}{|c|}{$\mathrm{g}-\mathrm{C}_{3} \mathrm{~N}_{4} / \mathrm{CDs} / \mathrm{BiOCl}$} \\
\hline Refluxing & $\begin{array}{c}\mathrm{C}_{3} \mathrm{H}_{6} \mathrm{~N}_{6} \\
\mathrm{Bi}\left(\mathrm{NO}_{3}\right)_{3} \cdot 5 \mathrm{H}_{2} \mathrm{O} \\
\mathrm{NaCl}, \mathrm{CH}_{4} \mathrm{~N}_{2} \mathrm{O} \\
\text { and } \mathrm{C}_{6} \mathrm{H}_{8} \mathrm{O}_{7}\end{array}$ & $\begin{array}{l}\text { Smaller spherical } \\
\text { particles of } \mathrm{CDs} \\
\text { and rod-like } \\
\text { particles of } \mathrm{BiOCl} \\
\text { are grown on the } \\
\text { surface of g- } \mathrm{C}_{3} \mathrm{~N}_{4} \\
\text { nanosheets }\end{array}$ & $\begin{array}{c}\text { Rhodamine B } \\
\left(1 \times 10^{-5} \mathrm{M}\right) \\
\text { Methylene } \\
\text { blue }\left(1 \times 10^{-5}\right. \\
\mathrm{M}) \\
\text { Methyl orange } \\
\left(1 \times 10^{-5} \mathrm{M}\right) \\
\text { Fuchsine }(0.77 \\
\left.\times 10^{-5} \mathrm{M}\right) \\
\text { Phenol }(5 \times \\
\left.10^{-5} \mathrm{M}\right)\end{array}$ & $\begin{array}{l}50 \mathrm{~W} \text { LED } \\
\text { lamp }\end{array}$ & $\begin{array}{c}\text { Ternary } \\
\text { heterojunction }\end{array}$ & $\begin{array}{c}\text { Formation of g- } \\
\mathrm{C}_{3} \mathrm{~N}_{4} / \mathrm{CDs} / \mathrm{BiOCl} \\
\text { composite } \\
\text { influenced the } \\
\text { optical properties } \\
\text { and photocatalytic } \\
\text { performance }\end{array}$ & [93] \\
\hline \multicolumn{8}{|c|}{$\mathrm{BiOBr} / \mathrm{rGO} / \mathrm{pg}-\mathrm{C}_{3} \mathrm{~N}_{4}$} \\
\hline Solvothermal & $\begin{array}{l}\mathrm{CH}_{4} \mathrm{~N}_{2} \mathrm{O}, \mathrm{HCl}, \\
\mathrm{rGO}, \mathrm{C}_{2} \mathrm{H}_{6} \mathrm{O}_{2}, \\
\mathrm{Bi}\left(\mathrm{NO}_{3}\right)_{3} \cdot 5 \mathrm{H}_{2} \mathrm{O} \\
\quad \text { and CTAB }\end{array}$ & $\begin{array}{l}\mathrm{BiOBr} \text { and } \mathrm{rGO} \\
\text { nanosheets are } \\
\text { dispersed } \\
\text { simultaneously on } \\
\text { the surface of } \\
\text { pg- } \mathrm{C}_{3} \mathrm{~N}_{4}\end{array}$ & $\begin{array}{l}\text { Rhodamine B } \\
\text { (10 ppm) } \\
\text { Tetracycline } \\
\text { (10 ppm) }\end{array}$ & $300 \mathrm{~W}$ Xe lamp & $\begin{array}{l}\text { Ternary } \\
\text { Z-scheme }\end{array}$ & $\begin{array}{l}\text { Optimized content } \\
\text { of } \mathrm{BiOBr} \text { in ternary } \\
\text { composite for high } \\
\text { activity was found } \\
\text { to be } 10 \mathrm{wt} \%\end{array}$ & [144] \\
\hline
\end{tabular}


Table 1. Cont.

\begin{tabular}{|c|c|c|c|c|c|c|c|}
\hline $\begin{array}{l}\text { Synthesis } \\
\text { Method }\end{array}$ & Precursors & Morphology & $\begin{array}{l}\text { Contaminant } \\
\text { Parameters }\end{array}$ & Light Source & $\begin{array}{c}\text { Heterojunction } \\
\text { Type }\end{array}$ & $\begin{array}{c}\text { Significance of the } \\
\text { Result }\end{array}$ & Ref. \\
\hline \multicolumn{8}{|c|}{$\mathrm{BiOBr} / \mathrm{CDs} / \mathrm{g}-\mathrm{C}_{3} \mathrm{~N}_{4}$} \\
\hline Hydrothermal & $\begin{array}{c}\mathrm{Bi}\left(\mathrm{NO}_{3}\right)_{3} \cdot 5 \mathrm{H}_{2} \mathrm{O} \\
\mathrm{KBr}, \mathrm{HNO}_{3} \\
\mathrm{CH}_{4} \mathrm{~N}_{2} \mathrm{O}, \mathrm{NH}_{4} \mathrm{Cl} \\
\mathrm{C}_{6} \mathrm{H}_{8} \mathrm{O}_{7} \text { and } \\
\mathrm{C}_{2} \mathrm{H}_{4} \mathrm{~N}_{4}\end{array}$ & $\begin{array}{l}\text { Ultrathin } \\
\text { nanosheets }\end{array}$ & $\begin{array}{l}\text { Ciprofloxacin } \\
\text { (10 ppm) } \\
\text { Tetracycline } \\
\text { (20 ppm) }\end{array}$ & $300 \mathrm{~W}$ Xe lamp & Z-scheme & $\begin{array}{l}\text { Up-converted PL } \\
\text { character and short } \\
\text { charge transport } \\
\text { distance of CDs } \\
\text { were beneficial } \\
\text { towards broadened } \\
\text { light absorption } \\
\text { and remarkable } \\
\text { interfacial charge } \\
\text { transfer }\end{array}$ & [145] \\
\hline \multicolumn{8}{|c|}{$\mathrm{CNNs} / \mathrm{CDs} / \mathrm{BiOBr}$} \\
\hline Refluxing & $\begin{array}{c}\mathrm{C}_{3} \mathrm{H}_{6} \mathrm{~N}_{6}, \mathrm{C}_{6} \mathrm{H}_{8} \mathrm{O}_{7} \\
\mathrm{CH}_{4} \mathrm{~N}_{2} \mathrm{O} \\
\mathrm{Bi}\left(\mathrm{NO}_{3}\right)_{3} \cdot 5 \mathrm{H}_{2} \mathrm{O} \\
\text { and } \mathrm{NaBr}\end{array}$ & $\begin{array}{l}\mathrm{CDs} \text { and } \mathrm{BiOBr} \\
\text { nanoparticles are } \\
\text { accumulated on } \\
\text { the surface of } \\
\text { carbon nitride } \\
\text { nanosheets (CNNs) }\end{array}$ & $\begin{array}{c}\text { Rhodamine B } \\
\left(1 \times 10^{-5} \mathrm{M}\right) \\
\text { Methylene } \\
\text { blue }\left(1 \times 10^{-5}\right. \\
\mathrm{M}) \\
\text { Methyl orange } \\
\left(1 \times 10^{-5} \mathrm{M}\right) \\
\text { Cr(VI) }(100 \\
\text { ppm })\end{array}$ & $\begin{array}{l}50 \text { W LED } \\
\text { lamp }\end{array}$ & $\begin{array}{l}\text { Ternary } \\
\text { Z-scheme }\end{array}$ & $\begin{array}{l}\mathrm{CNNs} / \mathrm{CDs} / \mathrm{BiOBr} \\
\text { was stable even } \\
\text { after five } \\
\text { consecutive cycles } \\
\text { towards the } \\
\text { degradation of } \\
\text { pollutants with } \\
\text { fresh dye solution } \\
\text { each time }\end{array}$ & [94] \\
\hline \multicolumn{8}{|c|}{$\mathrm{g}-\mathrm{C}_{3} \mathrm{~N}_{4} / \mathrm{BiOBr}-\mathrm{rGO}$} \\
\hline $\begin{array}{l}\text { Two-step hy- } \\
\text { drothermal } \\
\text { assembly } \\
\text { route }\end{array}$ & $\begin{array}{c}\text { Graphite powder, } \\
\mathrm{C}_{8} \mathrm{H}_{11} \mathrm{NO}_{2}, \mathrm{H}_{2} \mathrm{SO}_{4} \\
\mathrm{HNO}_{3}, \mathrm{KMnO}_{4} \\
\mathrm{H}_{2} \mathrm{O}_{2} \\
\mathrm{Bi}\left(\mathrm{NO}_{3}\right)_{3} \cdot 5 \mathrm{H}_{2} \mathrm{O} \\
\mathrm{KBr} \text { and } \mathrm{C}_{3} \mathrm{H}_{6} \mathrm{~N}_{6}\end{array}$ & $\begin{array}{l}\text { Flake-like } \mathrm{BiOBr} \\
\text { are covered by thin } \\
\text { layer of } \mathrm{g}-\mathrm{C}_{3} \mathrm{~N}_{4} \\
\text { film }\end{array}$ & $\begin{array}{l}\text { Rhodamine B } \\
\text { (10 ppm) }\end{array}$ & $300 \mathrm{~W}$ Xe lamp & $\mathrm{p}-\mathrm{n}$ & $\begin{array}{l}\text { Immobilization of } \\
\text { the powder catalyst } \\
\text { on 3D RGO aerogel } \\
\text { surface collectively } \\
\text { contributed to } \\
\text { excellent recycling } \\
\text { process of the } \\
\text { catalyst }\end{array}$ & [146] \\
\hline \multicolumn{8}{|c|}{ Carbon Fibers $/ \mathrm{g}-\mathrm{C}_{3} \mathrm{~N}_{4} / \mathrm{BiOBr}$} \\
\hline $\begin{array}{l}\text { Chemical } \\
\text { bath } \\
\text { deposition }\end{array}$ & $\begin{array}{c}\text { Carbon fibres, } \\
\mathrm{CH}_{4} \mathrm{~N}_{2} \mathrm{O} \\
\mathrm{Bi}\left(\mathrm{NO}_{3}\right)_{3} \cdot 5 \mathrm{H}_{2} \mathrm{O} \\
\mathrm{C}_{4} \mathrm{H}_{9} \mathrm{NO} \text { and } \mathrm{KBr}\end{array}$ & $\begin{array}{c}\text { Growth of } \mathrm{g}-\mathrm{C}_{3} \mathrm{~N}_{4} \\
\text { nanosheets and } \\
\mathrm{BiOBr} \text { nanoplates } \\
\text { on carbon fibers } \\
\text { (CFs) }\end{array}$ & $\begin{array}{l}\text { Tetracycline } \\
(20 \mathrm{ppm})\end{array}$ & $300 \mathrm{~W}$ Xe lamp & $\begin{array}{c}\text { Ternary } \\
\text { heterojunction }\end{array}$ & $\begin{array}{c}\text { Recyclable } \\
\text { cloth-shaped } \\
\mathrm{CFs} / \mathrm{g}- \\
\mathrm{C}_{3} \mathrm{~N}_{4} / \mathrm{BiOBr} \\
\text { bundles had great } \\
\text { mechanical } \\
\text { strength }\end{array}$ & [147] \\
\hline \multicolumn{8}{|c|}{$\mathrm{BiOBr} / \mathrm{CS} / \mathrm{g}-\mathrm{C}_{3} \mathrm{~N}_{4}$} \\
\hline Solvothermal & $\begin{array}{c}\mathrm{C}_{3} \mathrm{H}_{6} \mathrm{~N}_{6}, \mathrm{C}_{6} \mathrm{H}_{12} \mathrm{O}_{6} \\
\mathrm{Bi}\left(\mathrm{NO}_{3}\right)_{3} \cdot 5 \mathrm{H}_{2} \mathrm{O} \\
\mathrm{KBr} \text { and } \mathrm{C}_{2} \mathrm{H}_{6} \mathrm{O}_{2}\end{array}$ & $\begin{array}{l}\text { Spherical carbon } \\
\text { spheres are } \\
\text { wrapped uniformly } \\
\text { with } \mathrm{g}-\mathrm{C}_{3} \mathrm{~N}_{4} \text { and } \\
\mathrm{BiOBr} \text { matrix }\end{array}$ & $\begin{array}{l}\text { Rhodamine B } \\
\text { (10 ppm) }\end{array}$ & $\begin{array}{c}300 \mathrm{~W} W \\
\text { halogen lamp }\end{array}$ & $\begin{array}{c}\text { Ternary } \\
\text { heterojunction }\end{array}$ & $\begin{array}{l}\text { Carbon spheres } \\
\text { were used as } \\
\text { interlinking } \\
\text { network between } \\
\mathrm{g}-\mathrm{C}_{3} \mathrm{~N}_{4} \text { and } \mathrm{BiOBr} \\
\text { matrix for effective } \\
\text { electron transfer }\end{array}$ & [148] \\
\hline \multicolumn{8}{|c|}{$\mathrm{BiOI} /$ porous g- $\mathrm{C}_{3} \mathrm{~N}_{4}$ / graphene hydrogel } \\
\hline Hydrothermal & $\begin{array}{l}\mathrm{Bi}\left(\mathrm{NO}_{3}\right)_{3} \cdot 5 \mathrm{H}_{2} \mathrm{O}, \mathrm{KI} \\
\mathrm{CH}_{4} \mathrm{~N}_{2} \mathrm{O}, \mathrm{C}_{2} \mathrm{H}_{6} \mathrm{O}_{2}\end{array}$ & $\begin{array}{l}\mathrm{BiOI} \text { and porous } \\
\mathrm{g}-\mathrm{C}_{3} \mathrm{~N}_{4} \text { were } \\
\text { loaded onto } 3 \mathrm{D} \\
\text { cross-linking } \\
\text { graphene hydrogel }\end{array}$ & $\begin{array}{l}\text { Methylene } \\
\text { blue (40 ppm) } \\
\text { Levofloxacin } \\
(20 \text { ppm })\end{array}$ & $300 \mathrm{~W}$ Xe lamp & $\begin{array}{c}\text { Ternary } \\
\text { heterojunction }\end{array}$ & $\begin{array}{l}\text { 3D graphene } \\
\text { hydrogel played } \\
\text { multiple roles: } \\
\text { enhanced } \\
\text { adsorption ability, } \\
\text { provided bulk } \\
\text { electron transfer } \\
\text { channels, rendered } \\
\text { easy separation } \\
\text { and recycling }\end{array}$ & [149] \\
\hline
\end{tabular}


Table 1. Cont.

\begin{tabular}{|c|c|c|c|c|c|c|c|}
\hline $\begin{array}{l}\text { Synthesis } \\
\text { Method }\end{array}$ & Precursors & Morphology & $\begin{array}{l}\text { Contaminant } \\
\text { Parameters }\end{array}$ & Light Source & $\begin{array}{c}\text { Heterojunction } \\
\text { Type }\end{array}$ & $\begin{array}{c}\text { Significance of the } \\
\text { Result }\end{array}$ & Ref. \\
\hline \multicolumn{8}{|c|}{ Semiconductor coupled BiOX-g- $\mathrm{C}_{3} \mathrm{~N}_{4}$} \\
\hline \multicolumn{8}{|c|}{ Polyacrylonitrile/g- $\mathrm{C}_{3} \mathrm{~N}_{4} / \mathrm{BiOI}$ nanofibres } \\
\hline Impregnation & $\begin{array}{c}\mathrm{Bi}\left(\mathrm{NO}_{3}\right)_{3} \cdot 5 \mathrm{H}_{2} \mathrm{O}, \mathrm{KI}, \\
\mathrm{C}_{3} \mathrm{H}_{6} \mathrm{~N}_{6}, \mathrm{~N}, \mathrm{~N}- \\
\text { dimethylformamide } \\
\left(\mathrm{C}_{3} \mathrm{H}_{7} \mathrm{NO}\right) \text { and } \\
\text { polyacrylonitrile } \\
\left(\mathrm{C}_{3} \mathrm{H}_{3} \mathrm{~N}\right)_{\mathrm{n}}\end{array}$ & $\begin{array}{c}\mathrm{BiOI} \\
\text { nanostructures are } \\
\text { uniformly } \\
\text { dispersed over } \\
\mathrm{PAN} / \mathrm{g}-\mathrm{C}_{3} \mathrm{~N}_{4} \\
\text { nanofibres }\end{array}$ & $\begin{array}{l}\text { Rhodamine B } \\
\text { (10 ppm) } \\
\text { Cr (VI) }(20 \\
\text { ppm) }\end{array}$ & $300 \mathrm{~W}$ Xe lamp & - & $\begin{array}{c}\text { Ultralong 1D } \\
\text { macroscopic } \\
\text { flexible } \\
\text { self-supporting } \\
\text { floating structures } \\
\text { prevented } \\
\text { agglomeration and } \\
\text { loss of catalyst } \\
\text { during recycling }\end{array}$ & [150] \\
\hline \multicolumn{8}{|c|}{$\mathrm{SiO}_{2} @ \mathrm{~g}-\mathrm{C}_{3} \mathrm{~N}_{4} / \mathrm{BiOI}$ nanofibres } \\
\hline Impregnation & $\begin{array}{l}\text { Polyvinylpyrrolidone } \\
\left(\mathrm{C}_{6} \mathrm{H}_{9} \mathrm{NO}\right)_{\mathrm{n}}, \mathrm{TEOS} \\
\left(\mathrm{SiC}_{8} \mathrm{H}_{20} \mathrm{O}_{4}\right) \\
\text { ethanol, } \mathrm{C}_{3} \mathrm{H}_{6} \mathrm{~N}_{6} \\
\mathrm{Bi}\left(\mathrm{NO}_{3}\right)_{3} \cdot 5 \mathrm{H}_{2} \mathrm{O} \\
\text { and } \mathrm{KI}\end{array}$ & $\begin{array}{l}\text { BiOI nanosheets } \\
\text { are loaded on the } \\
\text { surface of ultrathin } \\
\text { g- } \mathrm{C}_{3} \mathrm{~N}_{4} @ \mathrm{SiO}_{2} \\
\text { nanofibres. }\end{array}$ & $\begin{array}{l}\text { Rhodamine B } \\
\text { (10 ppm) }\end{array}$ & 150 W Xe lamp & $\begin{array}{c}\text { Direct } \\
\text { Z-scheme }\end{array}$ & $\begin{array}{c}\text { Depositing } \mathrm{SiO}_{2} \\
\mathrm{NFs} \text { at } \\
\mathrm{BiOI} / \mathrm{g}-\mathrm{C}_{3} \mathrm{~N}_{4} \\
\text { interface improved } \\
\text { Z-scheme charge } \\
\text { carrier separation } \\
\text { and recyclability }\end{array}$ & [151] \\
\hline \multicolumn{8}{|c|}{$\mathrm{BiOI} / \mathrm{AgI} / \mathrm{g}-\mathrm{C}_{3} \mathrm{~N}_{4}$} \\
\hline $\begin{array}{l}\text { In situ crys- } \\
\text { tallization }\end{array}$ & $\begin{array}{c}\mathrm{Bi}\left(\mathrm{NO}_{3}\right)_{3} \cdot 5 \mathrm{H}_{2} \mathrm{O}, \mathrm{KI} \\
\mathrm{AgNO}_{3} \text { and } \\
\mathrm{C}_{3} \mathrm{H}_{6} \mathrm{~N}_{6}\end{array}$ & $\begin{array}{c}\text { Irregular } \\
\text { nanoparticles of } \\
\text { AgI are grown on } \\
\text { the surface of } \\
\text { g- } \mathrm{C}_{3} \mathrm{~N}_{4} \text { covered } \\
\text { with BiOI } \\
\text { nanoflakes. }\end{array}$ & $\begin{array}{l}\text { Methyl orange } \\
\text { (10 ppm) } \\
\text { Cr(VI) }(50 \\
\text { ppm) }\end{array}$ & $300 \mathrm{~W}$ Xe lamp & $\begin{array}{c}\text { Ternary } \\
\text { heterojunction }\end{array}$ & $\begin{array}{l}\text { Visible light } \\
\text { response was } \\
\text { tailored from } 460 \text { to } \\
560 \mathrm{~nm} \text { by } \\
\text { increasing the } \\
\text { content of AgI in } \\
\text { the composite }\end{array}$ & [152] \\
\hline \multicolumn{8}{|c|}{$\mathrm{BiOI} / \mathrm{g}-\mathrm{C}_{3} \mathrm{~N}_{4} / \mathrm{CeO}_{2}$} \\
\hline $\begin{array}{l}\text { Calcination } \\
\text { and hy- } \\
\text { drothermal }\end{array}$ & $\begin{array}{c}\mathrm{Bi}\left(\mathrm{NO}_{3}\right)_{3} \cdot 5 \mathrm{H}_{2} \mathrm{O}, \mathrm{KI} \\
\mathrm{Ce}\left(\mathrm{NO}_{3}\right)_{3} \cdot 6 \mathrm{H}_{2} \mathrm{O} \\
\text { and } \mathrm{C}_{3} \mathrm{H}_{6} \mathrm{~N}_{6}\end{array}$ & $\begin{array}{l}\mathrm{BiOI} \text { microspheres } \\
\text { and } \mathrm{CeO}_{2} \\
\text { nanoparticles are } \\
\text { randomly adhered } \\
\text { to the surface of } \\
{\mathrm{g}-\mathrm{C}_{3} \mathrm{~N}_{4}}\end{array}$ & $\begin{array}{l}\text { Tetracycline } \\
(20 \mathrm{ppm})\end{array}$ & 300 W Xe lamp & $\begin{array}{c}\text { Ternary } \\
\text { heterojunction }\end{array}$ & $\begin{array}{l}\text { Optimum content } \\
\text { of } \mathrm{CeO}_{2} \text { in the } \\
\text { ternary hybrid was } \\
\text { found to be } 3 \mathrm{wt} \% \\
\text { towards efficient } \\
\text { TC degradation }\end{array}$ & [153] \\
\hline \multicolumn{8}{|c|}{ BiOI@MIL-88A(Fe)@g-C $\mathrm{C}_{3}$} \\
\hline Hydrothermal & $\begin{array}{c}\mathrm{Bi}\left(\mathrm{NO}_{3}\right)_{3} \cdot 5 \mathrm{H}_{2} \mathrm{O}, \mathrm{KI} \\
\mathrm{C}_{2} \mathrm{H}_{6} \mathrm{O}_{2}, \mathrm{C}_{3} \mathrm{H}_{6} \mathrm{~N}_{6} \\
\mathrm{FeCl}_{3} \cdot 6 \mathrm{H}_{2} \mathrm{O} \text { and } \\
\mathrm{C}_{4} \mathrm{H}_{4} \mathrm{O}_{4}\end{array}$ & $\begin{array}{l}\text { BiOI flower-like } \\
\text { hierarchical } \\
\text { microspheres are } \\
\text { loaded on the } \\
\text { surface of MIL- } \\
88 \mathrm{~A}(\mathrm{Fe}) @ g-\mathrm{C}_{3} \mathrm{~N}_{4} \\
\text { with core@shell } \\
\text { structure }\end{array}$ & $\begin{array}{l}\text { Acid blue } 92 \\
\text { (10 ppm) } \\
\text { Rhodamine B } \\
\text { (10 ppm) } \\
\text { Phenol (10 } \\
\text { ppm) }\end{array}$ & $300 \mathrm{~W}$ Xe lamp & $\begin{array}{c}\text { Ternary } \\
\text { heterojunction }\end{array}$ & $\begin{array}{c}\text { g- } \mathrm{C}_{3} \mathrm{~N}_{4} \text { deposited } \\
\text { over } \\
\text { BiOI@MIL- } 88 \mathrm{~A}(\mathrm{Fe}) \\
\text { via hydrothermal } \\
\text { method facilitated } \\
\text { carrier separation } \\
\text { in the composite }\end{array}$ & [154] \\
\hline \multicolumn{8}{|c|}{$\mathrm{g}-\mathrm{C}_{3} \mathrm{~N}_{4} / \mathrm{Fe}_{3} \mathrm{O}_{4} / \mathrm{BiOI}$} \\
\hline $\begin{array}{l}\text { Reflux and } \\
\text { precipitation }\end{array}$ & $\begin{array}{c}\mathrm{Bi}\left(\mathrm{NO}_{3}\right)_{3} \cdot 4 \mathrm{H}_{2} \mathrm{O} \\
\mathrm{NaI}, \mathrm{C}_{3} \mathrm{H}_{6} \mathrm{~N}_{6} \\
\mathrm{FeCl} \cdot 6 \mathrm{H}_{2} \mathrm{O} \\
\mathrm{FeCl}_{2} \cdot 4 \mathrm{H}_{2} \mathrm{O} \text { and } \\
\mathrm{NH}_{3}\end{array}$ & $\begin{array}{c}\mathrm{Fe}_{3} \mathrm{O}_{4} \text { particles and } \\
\mathrm{BiOI} \text { are grown on } \\
\text { the surface of } \\
\mathrm{g}-\mathrm{C}_{3} \mathrm{~N}_{4} \text { sheets }\end{array}$ & $\begin{array}{c}\text { Rhodamine B } \\
\left(1 \times 10^{-5} \mathrm{M}\right) \\
\text { Methylene } \\
\text { blue }(1.3 \times \\
\left.10^{-5} \mathrm{M}\right) \\
\text { Methyl orange } \\
\left(1.05 \times 10^{-5}\right. \\
\mathrm{M})\end{array}$ & $\begin{array}{l}50 \text { W LED } \\
\text { source }\end{array}$ & $\begin{array}{c}\text { Ternary } \\
\text { heterojunction }\end{array}$ & $\begin{array}{c}\text { g- } \\
\mathrm{C}_{3} \mathrm{~N}_{4} / \mathrm{Fe}_{3} \mathrm{O}_{4} / \mathrm{BiOI} \\
\text { was magnetically } \\
\text { separated from the } \\
\text { aqueous medium } \\
\text { within a short span } \\
\text { of time }\end{array}$ & [92] \\
\hline \multicolumn{8}{|c|}{$\mathrm{g}-\mathrm{C}_{3} \mathrm{~N}_{4} / \mathrm{I}^{3-}-\mathrm{BiOI}$} \\
\hline Solvothermal & $\begin{array}{c}\mathrm{C}_{3} \mathrm{H}_{6} \mathrm{~N}_{6}, \\
\mathrm{Bi}_{6}\left(\mathrm{NO}_{3}\right)_{3} \cdot 4 \mathrm{H}_{2} \mathrm{O} \\
\mathrm{C}_{4} \mathrm{H}_{6} \mathrm{O}_{6}, \mathrm{C}_{4} \mathrm{H}_{10} \mathrm{O} \\
\mathrm{EDTA}^{-}-\mathrm{Na}_{1} \\
\mathrm{C}_{6} \mathrm{H}_{8} \mathrm{O}_{6}, \mathrm{~K}_{2} \mathrm{Cr}_{2} \mathrm{O}_{7} \\
\mathrm{NaN}_{3}, \mathrm{DMPO} \text { and } \\
\text { DMSO }\end{array}$ & $\begin{array}{c}\text { Flower-like } \\
\text { microspheres } \\
\text { containing } \\
\text { ultrathin } \\
\text { nanosheets of } \mathrm{BiOI} \\
\text { are loaded g- } \mathrm{C}_{3} \mathrm{~N}_{4}\end{array}$ & $\begin{array}{c}\text { Methyl } \\
\text { mercaptan } \\
\left(\mathrm{CH}_{3} \mathrm{SH}\right)(70 \\
\text { ppm })\end{array}$ & $8 \mathrm{~W}$ LED & Z-scheme & $\begin{array}{l}\mathrm{CH}_{3} \mathrm{SH} \text { removal } \\
\text { monitored via in } \\
\text { situ DRIFTS and } \\
\text { the intermediate } \\
\text { and conversion } \\
\text { pathways were } \\
\text { elucidated }\end{array}$ & [63] \\
\hline
\end{tabular}


Table 1. Cont

\begin{tabular}{|c|c|c|c|c|c|c|c|}
\hline $\begin{array}{l}\text { Synthesis } \\
\text { Method }\end{array}$ & Precursors & Morphology & $\begin{array}{l}\text { Contaminant } \\
\text { Parameters }\end{array}$ & Light Source & $\begin{array}{c}\text { Heterojunction } \\
\text { Type }\end{array}$ & $\begin{array}{c}\text { Significance of the } \\
\text { Result }\end{array}$ & Ref. \\
\hline \multicolumn{8}{|c|}{$\mathrm{MoS}_{2} / \mathrm{g} \mathrm{C}_{3} \mathrm{~N}_{4} / \mathrm{Bi}_{24} \mathrm{O}_{31} \mathrm{Cl}_{10}$} \\
\hline $\begin{array}{l}\text { Impregnation- } \\
\text { calcination }\end{array}$ & $\begin{array}{c}\mathrm{Bi}\left(\mathrm{NO}_{3}\right)_{3} \cdot 5 \mathrm{H}_{2} \mathrm{O} \\
\mathrm{C}_{3} \mathrm{H}_{6} \mathrm{~N}_{6}, \mathrm{NH}_{4} \mathrm{Cl} \\
\mathrm{C}_{6} \mathrm{H}_{8} \mathrm{O}_{7}, \mathrm{HNO}_{3} \\
\mathrm{NH}_{3} \cdot \mathrm{H}_{2} \mathrm{O} \\
\left(\mathrm{NH}_{4}\right)_{6} \mathrm{Mo}_{7} \mathrm{O}_{24} \cdot 4 \mathrm{H}_{2} \mathrm{O} \\
\text { and DMF }\end{array}$ & $\begin{array}{l}\text { Numerous g- } \mathrm{C}_{3} \mathrm{~N}_{4} \\
\text { nanosheets and } \\
\text { flower-like } \mathrm{MoS}_{2} \\
\text { are grown and } \\
\text { combined with } \\
\text { irregular block-like } \\
\text { shapes of } \mathrm{BOC}\end{array}$ & $\begin{array}{l}\text { Tetracycline } \\
(20 \text { ppm) }\end{array}$ & $300 \mathrm{~W}$ Xe lamp & $\begin{array}{l}\text { Dual Z-scheme } \\
\text { ternary } \\
\text { heterojunction }\end{array}$ & $\begin{array}{c}\text { Carrier lifetime } \\
\text { was higher in } \\
\text { CN/MS/BOC } \\
(3.9782 \mathrm{~ns}) \\
\text { compared to BOC } \\
(1.0163 \mathrm{~ns})\end{array}$ & [155] \\
\hline \multicolumn{8}{|c|}{$\mathrm{BiOCl} / \mathrm{Bi}_{2} \mathrm{MoO}_{6} / \mathrm{g}-\mathrm{C}_{3} \mathrm{~N}_{4}$} \\
\hline Refluxing & $\begin{array}{l}\mathrm{Bi}\left(\mathrm{NO}_{3}\right)_{3} \cdot 5 \mathrm{H}_{2} \mathrm{O} \\
\mathrm{Na}_{2} \mathrm{MoO}_{4} \cdot 2 \mathrm{H}_{2} \\
\mathrm{HCl} \text { and } \mathrm{NaOH}\end{array}$ & $\begin{array}{l}\text { Combination of } \\
\text { irregular rodlike, } \\
\text { platelet-shaped } \\
\text { and sheet-shaped } \\
\text { morphologies }\end{array}$ & $\begin{array}{l}\text { Rhodamine B } \\
\quad(0.5 \mathrm{mM})\end{array}$ & $350 \mathrm{~W}$ Xe lamp & $\begin{array}{c}\text { Ternary } \\
\text { heterojunction }\end{array}$ & $\begin{array}{c}\mathrm{BiOCl} / \mathrm{Bi}_{2} \mathrm{MoO}_{6} \\
\text { immobilized on } \\
\mathrm{g}-\mathrm{C}_{3} \mathrm{~N}_{4} \text { surface } \\
\text { exhibited dual } \\
\text { functionality as } \\
\text { photocatalysts and } \\
\text { optical limiters }\end{array}$ & [156] \\
\hline \multicolumn{8}{|c|}{$\mathrm{BiOCl} / \mathrm{CdS} / \mathrm{g}-\mathrm{C}_{3} \mathrm{~N}_{4}$} \\
\hline $\begin{array}{l}\text { Solvothermal } \\
\text { cum co- } \\
\text { precipitation }\end{array}$ & 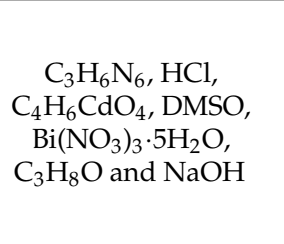 & $\begin{array}{c}\text { Growth of } \\
\text { hierarchical BiOCl } \\
\text { nanoflowers with } \\
\text { embedded CdS } \\
\text { nanoparticles on } \\
\text { g- } \mathrm{C}_{3} \mathrm{~N}_{4} \text { nanosheets }\end{array}$ & $\begin{array}{l}\text { Rhodamine B } \\
\text { (20 ppm) } \\
\text { Phenol }\end{array}$ & $\begin{array}{c}400 \mathrm{~W} \\
\text { Ne-illuminator }\end{array}$ & $\begin{array}{c}\text { Ternary } \\
\text { heterojunction }\end{array}$ & $\begin{array}{l}\text { Presence of two } \\
\text { visible light active } \\
\text { components led to } \\
\text { highly efficient } \\
\text { electron transfer in } \\
\text { multicomponent } \\
\text { heterojunction }\end{array}$ & [157] \\
\hline \multicolumn{8}{|c|}{$\mathrm{BiOCl} / \mathrm{g}-\mathrm{C}_{3} \mathrm{~N}_{4} /$ kaolinite } \\
\hline $\begin{array}{l}\text { Two-step } \\
\text { layer-by- } \\
\text { layer } \\
\text { self- } \\
\text { assembly }\end{array}$ & $\begin{array}{c}\mathrm{C}_{2} \mathrm{H}_{4} \mathrm{~N}_{4}, \mathrm{CTAC}, \\
\mathrm{Bi}\left(\mathrm{NO}_{3}\right)_{3} \cdot 5 \mathrm{H}_{2} \mathrm{O}, \\
\mathrm{CH}_{3} \mathrm{COOH} \\
\text { HCHO and } \\
\text { kaolinite }\end{array}$ & $\begin{array}{c}\mathrm{g}-\mathrm{C}_{3} \mathrm{~N}_{4} \text { and } \mathrm{BiOCl} \\
\text { ultrathin } \\
\text { nanosheets are } \\
\text { covered on the } \\
\text { surface of kaolinite } \\
\text { lamellar with } \\
\text { single layer }\end{array}$ & $\begin{array}{l}\text { Rhodamine B } \\
\text { (10 ppm) }\end{array}$ & $500 \mathrm{~W}$ Xe lamp & $\begin{array}{c}\text { Ternary } \\
\text { heterojunction }\end{array}$ & $\begin{array}{l}\text { Holes dominated } \\
\text { the degradation } \\
\text { pathways for } \\
\mathrm{BiOCl} / \mathrm{g}- \\
\mathrm{C}_{3} \mathrm{~N}_{4} / \text { kaolinite }\end{array}$ & [158] \\
\hline \multicolumn{8}{|c|}{$\mathrm{g}-\mathrm{C}_{3} \mathrm{~N}_{4} / \mathrm{g}-\mathrm{C}_{3} \mathrm{~N}_{4} / \mathrm{BiOBr}$} \\
\hline $\begin{array}{l}\text { Thermal de- } \\
\text { composition } \\
\text { and } \\
\text { solvother- } \\
\text { mal }\end{array}$ & $\begin{array}{c}\mathrm{CH}_{4} \mathrm{~N}_{2} \mathrm{O}, \mathrm{CH}_{4} \mathrm{~N}_{2} \mathrm{~S} \\
\mathrm{Bi}\left(\mathrm{NO}_{3}\right)_{3} \cdot 5 \mathrm{H}_{2} \mathrm{O} \\
\mathrm{KBr}, \mathrm{NaC}_{12} \mathrm{H}_{25} \mathrm{SO}_{4} \\
\text { and } \mathrm{C}_{2} \mathrm{H}_{6} \mathrm{O}_{2}\end{array}$ & $\begin{array}{c}\text { g- } \mathrm{C}_{3} \mathrm{~N}_{4} \text { prepared } \\
\text { using thiourea-urea } \\
\text { complex was } \\
\text { uniformly } \\
\text { dispersed on the } \\
\text { nanosheets of the } \\
\text { flower-like } \mathrm{BiOBr}\end{array}$ & $\begin{array}{l}\text { Rhodamine B } \\
\text { (20 ppm) } \\
\text { Fluorescein } \\
\text { isothiocyanate } \\
\text { (20 ppm) } \\
\text { Tetracycline } \\
\text { hydrochloride } \\
\text { (20 ppm) }\end{array}$ & $\begin{array}{l}\text { High pressure } \\
\text { Xe lamp }\end{array}$ & $\begin{array}{c}\text { Ternary direct } \\
\text { Z-scheme }+ \\
\text { isotype } \\
\text { heterojunction }\end{array}$ & 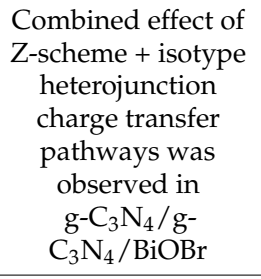 & [159] \\
\hline \multicolumn{8}{|c|}{$\mathrm{AgBr} / \mathrm{g}-\mathrm{C}_{3} \mathrm{~N}_{4} / \mathrm{BiOBr}$} \\
\hline $\begin{array}{l}\text { Hydrothermal } \\
\text { and in situ } \\
\text { ion- } \\
\text { exchange }\end{array}$ & $\begin{array}{c}\mathrm{CH}_{4} \mathrm{~N}_{2} \mathrm{O}, \mathrm{KBr}, \\
\mathrm{Bi}\left(\mathrm{NO}_{3}\right)_{3} \cdot 5 \mathrm{H}_{2} \mathrm{O}, \\
\mathrm{AgNO}_{3} \text { and } \\
\mathrm{C}_{2} \mathrm{H}_{6} \mathrm{O}_{2}\end{array}$ & $\begin{array}{c}\mathrm{AgBr} \text { nanoparticles } \\
\text { are dispersed on } \\
\text { the surface of } \\
\mathrm{g}-\mathrm{C}_{3} \mathrm{~N}_{4} / \mathrm{BiOBr} \\
\text { nanosheets }\end{array}$ & $\begin{array}{l}\text { Rhodamine B } \\
\text { (10 ppm) } \\
\text { Tetracycline } \\
\text { (10 ppm) }\end{array}$ & $300 \mathrm{~W}$ Xe lamp & $\begin{array}{c}\text { Ternary } \\
\text { heterojunction }\end{array}$ & $\begin{array}{l}\text { Influence of AgBr } \\
\text { loading on } \\
\text { GCN/BOB } \\
\text { composite towards } \\
\text { the photocatalytic } \\
\text { activity is } \\
\text { discussed in detail }\end{array}$ & [160] \\
\hline \multicolumn{8}{|c|}{ Brookite/g- $\mathrm{C}_{3} \mathrm{~N}_{4} / \mathrm{BiOBr}$} \\
\hline Hydrothermal & $\begin{array}{c}\mathrm{TiCl}_{4}, \\
\mathrm{Bi}\left(\mathrm{NO}_{3}\right)_{3} \cdot 5 \mathrm{H}_{2} \mathrm{O}, \\
\mathrm{C}_{3} \mathrm{H}_{6} \mathrm{~N}_{6}, \mathrm{CTAB}, \\
\mathrm{NH}_{4} \mathrm{Cl}, \mathrm{KBr} \text { and } \\
\mathrm{NaOH}\end{array}$ & $\begin{array}{l}\text { Spindle shaped } \\
\text { brookite are } \\
\text { wrapped by the } \\
\text { layer structure of } \\
\text { BiOBr which are } \\
\text { further wrapped } \\
\text { by lamellar g- } \mathrm{C}_{3} \mathrm{~N}_{4}\end{array}$ & $\begin{array}{l}\text { Rhodamine B } \\
\text { (10 ppm) }\end{array}$ & $\begin{array}{l}70 \mathrm{~W} \text { metal } \\
\text { halide lamp }\end{array}$ & $\begin{array}{c}\text { Ternary } \\
\text { heterojunction }\end{array}$ & $\begin{array}{l}\text { Ternary composite } \\
\text { had ability to } \\
\text { destroy the oxygen } \\
\text { heteroanthracene } \\
\text { ring and } \\
\text { chromogenic group } \\
\text { of RhB }\end{array}$ & [161] \\
\hline
\end{tabular}


Table 1. Cont

\begin{tabular}{|c|c|c|c|c|c|c|c|}
\hline $\begin{array}{l}\text { Synthesis } \\
\text { Method }\end{array}$ & Precursors & Morphology & $\begin{array}{l}\text { Contaminant } \\
\text { Parameters }\end{array}$ & Light Source & $\begin{array}{l}\text { Heterojunction } \\
\text { Type }\end{array}$ & $\begin{array}{c}\text { Significance of the } \\
\text { Result }\end{array}$ & Ref. \\
\hline \multicolumn{8}{|c|}{$\mathrm{BiOCl} / \mathrm{g}-\mathrm{C}_{3} \mathrm{~N}_{4} @ \mathrm{UiO}-66$} \\
\hline Solvothermal & $\begin{array}{c}\mathrm{Bi}\left(\mathrm{NO}_{3}\right)_{3} \cdot 5 \mathrm{H}_{2} \mathrm{O} \\
\mathrm{KCl}, \mathrm{CH}_{3} \mathrm{COOH} \\
\mathrm{C}_{3} \mathrm{H}_{6} \mathrm{~N}_{6}, \mathrm{ZrCl}_{4} \\
\mathrm{C}_{8} \mathrm{H}_{6} \mathrm{O}_{4}, \mathrm{C}_{3} \mathrm{H}_{7} \mathrm{NO}\end{array}$ & $\begin{array}{l}\mathrm{BiOCl} \text { nanoplates } \\
\text { and } \mathrm{g}-\mathrm{C}_{3} \mathrm{~N}_{4} \\
\text { nanosheets were } \\
\text { decorated over the } \\
\text { surface of } \mathrm{UiO}-66\end{array}$ & $\begin{array}{l}\text { Rhodamine B } \\
\text { (10 ppm) }\end{array}$ & $250 \mathrm{~W}$ Xe lamp & $\begin{array}{c}\text { Ternary } \\
\text { heterojunction }\end{array}$ & $\begin{array}{l}\text { UiO- } 66 \text { was proved } \\
\text { beneficial to the } \\
\text { photocatalytic } \\
\text { reaction by } \\
\text { enlarging the } \\
\text { photoadsorption } \\
\text { and preventing the } \\
\text { electron-hole } \\
\text { recombination }\end{array}$ & [121] \\
\hline \multicolumn{8}{|c|}{$\mathrm{g}-\mathrm{C}_{3} \mathrm{~N}_{4} / \mathrm{BiOI} / \mathrm{Bi}_{2} \mathrm{O}_{2} \mathrm{CO}_{3}$} \\
\hline $\begin{array}{l}\text { Simple reflux } \\
\text { and in situ } \\
\text { ion exchange }\end{array}$ & $\begin{array}{c}\mathrm{Bi}\left(\mathrm{NO}_{3}\right)_{3} \cdot 5 \mathrm{H}_{2} \mathrm{O}, \\
\mathrm{C}_{3} \mathrm{H}_{6} \mathrm{~N}_{6}, \mathrm{KI}, \\
\mathrm{NaHCO}_{3}, \mathrm{Na}_{2} \mathrm{SO}_{4}, \\
\mathrm{CH}_{3} \mathrm{CH}_{2} \mathrm{OH}, \\
\mathrm{Kr}_{2} \mathrm{Cr}_{2} \mathrm{O}_{7}, \mathrm{H}_{2} \mathrm{SO}_{4} \\
\mathrm{H}_{3} \mathrm{PO}_{4}\end{array}$ & $\begin{array}{c}\text { Thin nanosheets of } \\
\text { BiOI are } \\
\text { distributed over } \\
\text { g- } \mathrm{C}_{3} \mathrm{~N}_{4} \text { layers }\end{array}$ & $\begin{array}{l}\text { Rhodamine B } \\
\text { (10 ppm) }\end{array}$ & 250 W Xe lamp & $\begin{array}{c}\text { Ternary } \\
\text { heterojunction }\end{array}$ & $\begin{array}{l}\text { Based on the } \\
\text { matched energy } \\
\text { levels, BiOI acted } \\
\text { as the charge } \\
\text { transmission } \\
\text { bridge }\end{array}$ & [162] \\
\hline \multicolumn{8}{|c|}{$\mathrm{BiOX}$ and $\mathrm{BiOY}$ coupled $\mathrm{g}-\mathrm{C}_{3} \mathrm{~N}_{4}$} \\
\hline \multicolumn{8}{|c|}{$\mathrm{Bi}_{7} \mathrm{O}_{9} \mathrm{I}_{3} / \mathrm{Bi}_{5} \mathrm{O}_{7} \mathrm{I} / \mathrm{g}-\mathrm{C}_{3} \mathrm{~N}_{4}$} \\
\hline Hydrothermal & $\begin{array}{c}\mathrm{C}_{3} \mathrm{H}_{6} \mathrm{~N}_{6} \\
\mathrm{Bi}\left(\mathrm{NO}_{3}\right)_{3} \cdot 4 \mathrm{H}_{2} \mathrm{O}, \\
\mathrm{C}_{2} \mathrm{H}_{6} \mathrm{O}_{2} \text { and } \mathrm{KI}\end{array}$ & $\begin{array}{l}\text { Irregular rods } \\
\text { consisting of thin } \\
\text { irregular } \\
\text { nanosheets }\end{array}$ & $\begin{array}{l}\text { Crystal violet } \\
\text { (10 ppm) }\end{array}$ & $150 \mathrm{~W}$ Xe lamp & $\begin{array}{c}\text { Binary } \\
\text { heterojunction }\end{array}$ & $\begin{array}{c}\text { Controlled } \\
\text { synthesis of series } \\
\text { of } \mathrm{BiO}_{x} \mathrm{I}_{y} / g-\mathrm{C}_{3} \mathrm{~N}_{4} \\
\text { composites is } \\
\text { reported }\end{array}$ & [163] \\
\hline \multicolumn{8}{|c|}{$\mathrm{g}-\mathrm{C}_{3} \mathrm{~N}_{4} / \mathrm{BiOI} / \mathrm{BiOBr}$} \\
\hline $\begin{array}{l}\text { Chemical } \\
\text { precipitation }\end{array}$ & $\begin{array}{c}\mathrm{CH}_{4} \mathrm{~N}_{2} \mathrm{O}, \mathrm{C}_{2} \mathrm{H}_{6} \mathrm{O}_{2} \\
\mathrm{Bi}\left(\mathrm{NO}_{3}\right)_{3} \cdot 5 \mathrm{H}_{2} \mathrm{O} \\
\mathrm{KBr} \text { and } \mathrm{KI}\end{array}$ & $\begin{array}{c}\text { Curved } \mathrm{g}-\mathrm{C}_{3} \mathrm{~N}_{4} \\
\text { nanosheets are } \\
\text { attached to the } \\
\text { surface of } \\
\mathrm{BiOI} / \mathrm{BiOBr} \\
\text { exhibiting } \\
\text { sphere-like } \\
\text { structures } \\
\text { containing thin } \\
\text { nanosheets of } \mathrm{BiOI} \\
\text { on large plates of } \\
\mathrm{BiOBr}\end{array}$ & $\begin{array}{l}\text { Methyl orange } \\
\text { (10 ppm) } \\
\text { Escherichia } \\
\text { coli (ATCC } \\
15597)\end{array}$ & $300 \mathrm{~W}$ Xe lamp & $\begin{array}{c}\text { Ternary } \\
\text { heterojunction }\end{array}$ & $\begin{array}{l}\text { Presence of } \mathrm{BiOI} \\
\text { shifted the } \\
\text { bandgap to longer } \\
\text { wavelength and } \\
\text { also suppressed the } \\
\text { carrier } \\
\text { recombination }\end{array}$ & [164] \\
\hline \multicolumn{8}{|c|}{$\mathrm{g}-\mathrm{C}_{3} \mathrm{~N}_{4} @ \mathrm{BiOCl} / \mathrm{Bi}_{12} \mathrm{O}_{17} \mathrm{Cl}_{2}$} \\
\hline $\begin{array}{l}\text { In situ self- } \\
\text { assembly }\end{array}$ & $\begin{array}{c}\mathrm{CH}_{4} \mathrm{~N}_{2} \mathrm{O}, \mathrm{BiCl}_{3}, \\
\mathrm{C}_{2} \mathrm{H}_{5} \mathrm{OH} \text { and } \\
\mathrm{NaOH}\end{array}$ & $\begin{array}{l}\text { Combination of } \\
\text { layered and } \\
\text { irregular } \\
\text { microstructures } \\
\text { having smooth } \\
\text { nanosheets of } \\
\text { different sizes are } \\
\text { grown over g- } \mathrm{C}_{3} \mathrm{~N}_{4}\end{array}$ & $\begin{array}{l}\text { NO removal (1 } \\
\left.\times 10^{-9} \mathrm{ppb}\right)\end{array}$ & $\begin{array}{l}100 \mathrm{~W} \\
\text { commercial } \\
\text { tungsten } \\
\text { halogen lamp }\end{array}$ & $\begin{array}{c}\text { Ternary } \\
\text { heterojuncton }\end{array}$ & $\begin{array}{l}\text { Electron spin } \\
\text { resonance proved } \\
\text { that both hydroxyl } \\
\text { and superoxide } \\
\text { radicals are active } \\
\text { species towards } \\
\text { NO removal }\end{array}$ & [165] \\
\hline \multicolumn{8}{|c|}{$\mathrm{BiOI} / \mathrm{BiOCl} / \mathrm{g}-\mathrm{C}_{3} \mathrm{~N}_{4}$} \\
\hline Precipitation & $\begin{array}{c}\mathrm{Bi}\left(\mathrm{NO}_{3}\right)_{3} \cdot 5 \mathrm{H}_{2} \mathrm{O} \\
\mathrm{CH}_{4} \mathrm{~N}_{2} \mathrm{O}, \mathrm{KI}, \mathrm{KCl} \\
\quad \text { and } \mathrm{NH}_{3}\end{array}$ & $\begin{array}{l}\text { Nanosheets are } \\
\text { stacked densely to } \\
\text { form irregular } \\
\text { microstructures } \\
\text { over thin layers of } \\
\text { g- } \mathrm{C}_{3} \mathrm{~N}_{4}\end{array}$ & $\begin{array}{l}\text { Acid orange } \\
\text { (10 ppm) }\end{array}$ & $\begin{array}{l}400 \text { W Halogen } \\
\text { lamp }\end{array}$ & $\begin{array}{c}\text { Ternary } \\
\text { heterojuncton }\end{array}$ & $\begin{array}{c}\text { The optimal ratio } \\
\text { of ternary hybrid } \\
\text { was found to be } \\
5: 3: 2\end{array}$ & [91] \\
\hline
\end{tabular}


Table 1. Cont.

\begin{tabular}{|c|c|c|c|c|c|c|c|}
\hline $\begin{array}{l}\text { Synthesis } \\
\text { Method }\end{array}$ & Precursors & Morphology & $\begin{array}{l}\text { Contaminant } \\
\text { Parameters }\end{array}$ & Light Source & $\begin{array}{l}\text { Heterojunction } \\
\text { Type }\end{array}$ & $\begin{array}{c}\text { Significance of the } \\
\text { Result }\end{array}$ & Ref. \\
\hline \multicolumn{8}{|c|}{ Quaternary heterojunction } \\
\hline \multicolumn{8}{|c|}{$\mathrm{BiOCl} / \mathrm{g}-\mathrm{C}_{3} \mathrm{~N}_{4} / \mathrm{Cu}_{2} \mathrm{O} / \mathrm{Fe}_{3} \mathrm{O}_{4}$} \\
\hline $\begin{array}{c}\text { Co- } \\
\text { precipitation }\end{array}$ & $\begin{array}{c}\mathrm{Bi}\left(\mathrm{NO}_{3}\right)_{3} \cdot 5 \mathrm{H}_{2} \mathrm{O} \\
\mathrm{KCl}, \mathrm{NaOH} \mathrm{HNO}_{3}, \\
\mathrm{CuSO}_{4} \cdot 5 \mathrm{H}_{2} \mathrm{O} \\
\mathrm{C}_{2} \mathrm{H}_{5} \mathrm{OH}, \mathrm{FeCl}_{3}, \\
\mathrm{FeCl}_{2} \mathrm{CH}_{4} \mathrm{~N}_{2} \mathrm{~S} \text { and } \\
\mathrm{C}_{2} \mathrm{H}_{6} \mathrm{O}_{2}\end{array}$ & $\begin{array}{c}\text { Flower shaped } \\
\mathrm{BiOCl} \text {, spherical } \\
\mathrm{Fe}_{3} \mathrm{O}_{4} \text { and cubical } \\
\mathrm{Cu}_{2} \mathrm{O} \\
\text { nanoparticles are } \\
\text { connected with } \\
\text { porous sheets of } \\
\text { g- }-\mathrm{C}_{3} \mathrm{~N}_{4} \\
\end{array}$ & $\begin{array}{l}\text { Sulfamethoxazole } \\
\qquad(100 \mu \mathrm{M})\end{array}$ & $\begin{array}{l}800 \text { W Xe lamp } \\
\text { Natural } \\
\text { sunlight }\end{array}$ & $\begin{array}{c}\text { Quaternary } \\
\text { nano- } \\
\text { heterojunction }\end{array}$ & $\begin{array}{c}\text { p-n-p junction } \\
\text { functioned well } \\
\text { under both } \\
\text { artificial visible } \\
\text { light and solar light } \\
\text { towards } \\
\text { sulfamethoxazole } \\
\text { degradation }\end{array}$ & [166] \\
\hline \multicolumn{8}{|c|}{$\mathrm{g}-\mathrm{C}_{3} \mathrm{~N}_{4} / \mathrm{BiOI} / \mathrm{BiOBr}$} \\
\hline Solvothermal & $\begin{array}{c}\mathrm{C}_{2} \mathrm{H}_{4} \mathrm{~N}_{4}, \mathrm{C}_{2} \mathrm{H}_{6} \mathrm{O}_{2} \\
\mathrm{Bi}\left(\mathrm{NO}_{3}\right)_{3} \cdot 5 \mathrm{H}_{2} \mathrm{O}, \mathrm{KI} \\
\text { and CTAB }\end{array}$ & $\begin{array}{l}\text { g- } \mathrm{C}_{3} \mathrm{~N}_{4} \text { was } \\
\text { attached to the } \\
\text { surface of quadrate } \\
\text { BiOBr substrates } \\
\text { overlapped with } \\
\text { rounded thin } \\
\text { pieces of the BiOI }\end{array}$ & $\begin{array}{c}\text { Methylene } \\
\text { blue (20 ppm) }\end{array}$ & 500 W Xe lamp & $\begin{array}{l}\text { Ternary } \\
\text { Z-scheme }\end{array}$ & $\begin{array}{l}\text { Charge carrier } \\
\text { dynamics in } \\
\text { ternary composite } \\
\text { is reviewed based } \\
\text { on transient } \\
\text { photocurrent } \\
\text { response }\end{array}$ & [167] \\
\hline \multicolumn{8}{|c|}{ Doped BiOX-g- $\mathrm{C}_{3} \mathrm{~N}_{4}$} \\
\hline \multicolumn{8}{|c|}{ K-doped g- $\mathrm{C}_{3} \mathrm{~N}_{4} / \mathrm{BiOBr}$} \\
\hline $\begin{array}{l}\text { In situ } \\
\text { synthesis }\end{array}$ & $\begin{array}{c}\mathrm{CH}_{4} \mathrm{~N}_{2} \mathrm{O}, \mathrm{CTAB}, \\
\mathrm{KOH} \text { and } \\
\mathrm{Bi}\left(\mathrm{NO}_{3}\right)_{3} \cdot 5 \mathrm{H}_{2} \mathrm{O}\end{array}$ & 2D nanosheets & $\begin{array}{l}\text { Rhodamine B } \\
\text { (20 ppm) } \\
\text { Tetracycline } \\
\text { (10 ppm) }\end{array}$ & 500 W Xe lamp & $\begin{array}{c}\text { Binary } \\
\text { heterojunction }\end{array}$ & $\begin{array}{c}\mathrm{K} \text { was interfaced } \\
\text { with } \\
\mathrm{g}-\mathrm{C}_{3} \mathrm{~N}_{4} / \mathrm{BiOBr} \text { for } \\
\text { improved } \\
\text { migration and } \\
\text { transportation of } \\
\text { photogenic carriers }\end{array}$ & [168] \\
\hline \multicolumn{8}{|c|}{$\mathrm{g}-\mathrm{C}_{3} \mathrm{~N}_{4} @ \mathrm{Bi} / \mathrm{BiOBr}$} \\
\hline Solvothermal & $\begin{array}{c}\mathrm{C}_{3} \mathrm{H}_{6} \mathrm{~N}_{6} \\
\mathrm{Bi}\left(\mathrm{NO}_{3}\right)_{3} \cdot 5 \mathrm{H}_{2} \mathrm{O} \\
\mathrm{C}_{2} \mathrm{H}_{6} \mathrm{O}_{2}, \mathrm{KBr} \text { and } \\
\mathrm{C}_{2} \mathrm{H}_{5} \mathrm{OH}\end{array}$ & $\begin{array}{c}\text { 3D fluffy and } \\
\text { hierarchical } \\
\text { structure where } \\
\mathrm{Bi} / \mathrm{BiOBr} \\
\text { nanoplates are } \\
\text { embedded on the } \\
\text { surface of the } \\
\text { layered } \mathrm{g}-\mathrm{C}_{3} \mathrm{~N}_{4}\end{array}$ & $\begin{array}{l}\text { Rhodamine B } \\
\text { (20 ppm) } \\
\text { Tetracycline } \\
\text { (12 ppm) }\end{array}$ & $\begin{array}{l}\text { Simulated } \\
\text { sunlight }\end{array}$ & $\begin{array}{l}\text { Ternary } \\
\text { indirect } \\
\text { Z-scheme }\end{array}$ & $\begin{array}{l}\text { Ethylene glycol } \\
\text { functioned as } \\
\text { solvent and a } \\
\text { reductant for } \\
\text { tuning the } \\
\text { morphology and } \\
\text { boosting the } \\
\text { photocatalytic } \\
\text { performance }\end{array}$ & [84] \\
\hline \multicolumn{8}{|c|}{ g- $\mathrm{C}_{3} \mathrm{~N}_{4} @$ Polydopamine/BiOBr } \\
\hline Solvothermal & $\begin{array}{c}\mathrm{C}_{3} \mathrm{H}_{6} \mathrm{~N}_{6}, \mathrm{HCl}, \\
\mathrm{Da} . \mathrm{HCl}, \mathrm{NaOH}, \\
\mathrm{Bi}\left(\mathrm{NO}_{3}\right)_{3} \cdot 5 \mathrm{H}_{2} \mathrm{O}, \\
\mathrm{PVP}, \mathrm{C}_{2} \mathrm{H}_{6} \mathrm{O}_{2} \text { and } \\
\mathrm{KBr}\end{array}$ & $\begin{array}{l}\text { Flower-like BiOBr } \\
\text { are deposited on } \\
\text { the surface of } \\
\text { sheet-like } \\
\text { g- } \mathrm{C}_{3} \mathrm{~N}_{4} @ \mathrm{PDA}\end{array}$ & $\begin{array}{l}\text { Sulfamethoxazole } \\
\quad(2.5 \mathrm{ppm})\end{array}$ & 300 W Xe lamp & Z-scheme & $\begin{array}{l}\text { Biomimetic } \mathrm{PDA} \text { as } \\
\text { electron transfer } \\
\text { mediator bridging } \\
\mathrm{g}-\mathrm{C}_{3} \mathrm{~N}_{4}-\mathrm{BiOBr} \text { was } \\
\text { reported for the } \\
\text { first time }\end{array}$ & [169] \\
\hline
\end{tabular}

\subsection{Carbon Dioxide Reduction}

The rapid increase in the concentration of atmospheric $\mathrm{CO}_{2}$ as a green-house gas has drawn significant concerns over its huge impact on the global climate. Therefore, the photocatalytic reduction of $\mathrm{CO}_{2}$ to value-added chemicals such as $\mathrm{CO}, \mathrm{CH}_{3} \mathrm{OH}, \mathrm{HCOOH}$, $\mathrm{CH}_{4}$, etc., under direct solar irradiation is pivotal for not only reducing the level of atmospheric $\mathrm{CO}_{2}$, but also for partly fulfilling the renewable fuel demand that may increase in the future, partly owing to the steadily depleting fossil fuel reserves and also due to our environmental policy on curbing the usage of fossil fuels for inhibiting $\mathrm{CO}_{2}$ emission. As mentioned earlier, $\mathrm{BiOX}$ photocatalysts are mainly employed for the photocatalytic degradation of organic pollutants and are seldom effective in the reduction of $\mathrm{CO}_{2}$ conversion at neutral condition due to its positive $C B$ position [170]. Therefore, only a few photocatalytic reduction reactions of pristine $\mathrm{BiOX}$ for photocatalytic $\mathrm{CO}_{2}$ conversion have been reported 
to date $[90,171-179]$. On the other hand, theoretical studies indicated that the increase in the Bi-content in BiOX could promote the reduction power of photogenerated electrons and increase the thermodynamic force for initiating many reduction reactions that were not possible to be carried out with $\mathrm{BiOX}$. In this regard, non-stoichiometric $\mathrm{Bi}_{\mathrm{x}} \mathrm{O}_{\mathrm{y}} \mathrm{X}_{\mathrm{z}}$ photocatalysts were found to exhibit promising potential in the photoreduction of $\mathrm{CO}_{2}$ to solar fuels and exhibited good stability and possessed suitable band structures for extended visible light absorption with negative $\mathrm{CB}$ positions [180]. For instance, Ye et al. reported an enhanced rate of $\mathrm{CO}$ and $\mathrm{CH}_{4}$ generation by the photocatalytic reduction of $\mathrm{CO}_{2}$ using $\mathrm{Bi}_{4} \mathrm{O}_{5} \mathrm{Br}_{2}$ microspheres assembled with ultrathin nanosheets in comparison to $\mathrm{BiOBr}$ with ultrathin nanosheets and bulk $\mathrm{BiOBr}$. It was proved that $\mathrm{Bi}$-rich $\mathrm{Bi}_{4} \mathrm{O}_{5} \mathrm{Br}_{2}$ with a more negative $\mathrm{CB}$ position exhibited enhanced photoreduction of $\mathrm{CO}_{2}$ in comparison to $\mathrm{BiOBr}$. Further, it was revealed that the ultrathin nanosheet morphology of both $\mathrm{Bi}_{4} \mathrm{O}_{5} \mathrm{Br}_{2}$ and $\mathrm{BiOBr}$ considerably reduced the recombination due to IEF generation and supported the generation of $\mathrm{CO}$ in comparison to bulk $\mathrm{BiOBr}$ [181]. Similarly, ultrathin $\mathrm{Bi}_{4} \mathrm{O}_{5} \mathrm{Br}_{2}$ nanosheets synthesized through the molecular precursor method exhibited enhanced performance towards $\mathrm{CO}_{2}$ reduction under visible light irradiation in comparison to bulk $\mathrm{Bi}_{4} \mathrm{O}_{5} \mathrm{Br}_{2}$. The amount of $\mathrm{CO}_{2}$ converted to $\mathrm{CO}$ was $63.13 \mu \mathrm{mol} \mathrm{g}{ }^{-1}$ using $\mathrm{Bi}_{4} \mathrm{O}_{5} \mathrm{Br}_{2}$ ultrathin nanosheets, which was $\sim 2.3$ times greater than that of bulk of $\mathrm{Bi}_{4} \mathrm{O}_{5} \mathrm{Br}_{2}\left(27.56 \mu \mathrm{mol} \mathrm{g}{ }^{-1}\right)$ [182]. The reason for the enhanced $\mathrm{CO}_{2}$ reduction ability using $\mathrm{Bi}_{4} \mathrm{O}_{5} \mathrm{Br}_{2}$ ultrathin nanosheets in comparison to its bulk counterpart was attributed to porous architecture with larger surface area, more negative $\mathrm{CB}$ position $(-1.19 \mathrm{~V})$, lower rate of recombination of the photogenerated charge carriers and higher photocurrent response. In another study, $\mathrm{Bi}_{4} \mathrm{O}_{5} \mathrm{I}_{2}$ and $\mathrm{Bi}_{5} \mathrm{O}_{7} \mathrm{I}$ photocatalysts were successfully synthesized via hydrolyzation and calcination, respectively, using the molecular precursor method. Both $\mathrm{Bi}_{4} \mathrm{O}_{5} \mathrm{I}_{2}$ and $\mathrm{Bi}_{5} \mathrm{O}_{7} \mathrm{I}$ exhibited the photocatalytic reduction of $\mathrm{CO}_{2}$ to selectively generate $\mathrm{CO}$, but the higher $\mathrm{CB}$ edge and lower bandgap energy $(2.18 \mathrm{eV})$ of $\mathrm{Bi}_{4} \mathrm{O}_{5} \mathrm{I}_{2}$ enabled it to exhibit enhanced photocatalytic performance that was $\sim 11.5$ and $\sim 28.3$ times greater than that of $\mathrm{Bi}_{5} \mathrm{O}_{7} \mathrm{I}$ and BiOI, respectively [183]. In addition to Bi-rich strategy, the hybridization of $\mathrm{Bi}_{4} \mathrm{O}_{5} \mathrm{I}_{2}$ with $\mathrm{g}-\mathrm{C}_{3} \mathrm{~N}_{4}$ was employed for enhancing the photoreduction of $\mathrm{CO}_{2}$ by the formation of a heterojunction with an $\mathrm{I}_{3}{ }^{-} / \mathrm{I}^{-}$redox mediator synthesized through the complex precursor method. The composite exhibited higher photocatalytic activity for $\mathrm{CO}_{2}$ conversion than pure $\mathrm{g}-\mathrm{C}_{3} \mathrm{~N}_{4}$ and $\mathrm{Bi}_{4} \mathrm{O}_{5} \mathrm{I}_{2}$ owing to the $\mathrm{I}_{3}^{-} / \mathrm{I}^{-}$redox mediator formed in situ, which assisted the transfer of the photogenerated charge carriers through the Z-scheme heterojunction and suppressed their recombination [184]. The amount of $\mathrm{CO}$ generated by the photocatalytic reduction of $\mathrm{CO}_{2}$ in the presence of $\mathrm{g}-\mathrm{C}_{3} \mathrm{~N}_{4} / \mathrm{Bi}_{4} \mathrm{O}_{5} \mathrm{I}_{2}(20 \mathrm{wt} \%)$ with $45.6 \mu \mathrm{mol} \mathrm{g}^{-1} \mathrm{~h}^{-1}$ was $\sim 7.9$ and $\sim 2.3$ times greater than pristine g- $\mathrm{C}_{3} \mathrm{~N}_{4}$ and pristine $\mathrm{Bi}_{4} \mathrm{O}_{5} \mathrm{I}_{2}$, respectively. The performance of various $\mathrm{BiOX} / \mathrm{Bi}_{\mathrm{x}} \mathrm{O}_{\mathrm{y}} \mathrm{X}_{\mathrm{z}}-\mathrm{g}-\mathrm{C}_{3} \mathrm{~N}_{4}$ heterojunction photocatalysts for the reduction of $\mathrm{CO}_{2}$ is summarized in Table 2.

Table 2. Reduction of $\mathrm{CO}_{2}$ in the presence of $\mathrm{BiOX} / \mathrm{Bi}_{\mathrm{x}} \mathrm{O}_{\mathrm{y}} \mathrm{X}_{\mathrm{z}}-\mathrm{g}-\mathrm{C}_{3} \mathrm{~N}_{4}$ heterojunction photocatalysts reported in the literature.

\begin{tabular}{|c|c|c|c|c|}
\hline Photocatalyst & Light Source & Result & Significance & Ref. \\
\hline $\mathrm{g}-\mathrm{C}_{3} \mathrm{~N}_{4} / \mathrm{Bi}_{4} \mathrm{O}_{5} \mathrm{I}_{2}$ & $\begin{array}{c}300 \mathrm{~W} \text { Xe lamp } \\
(\lambda>420 \mathrm{~nm})\end{array}$ & $\begin{array}{l}\text { Photoreduction of } \mathrm{CO}_{2} \\
\mathrm{CO}-45.6 \mu \mathrm{mol} \mathrm{g}^{-1} \mathrm{~h}^{-1}\end{array}$ & $\begin{array}{c}\mathrm{I}_{3}^{-} / \mathrm{I}^{-} \text {redox mediator assisted } \mathrm{Z} \text {-scheme } \\
\text { mechanism enhanced the photocatalytic } \mathrm{CO}_{2} \\
\text { conversion }\end{array}$ & [184] \\
\hline $\mathrm{g}-\mathrm{C}_{3} \mathrm{~N}_{4} / \mathrm{BiOI}$ & $\begin{array}{l}300 \mathrm{~W} \text { Xe lamp } \\
(\lambda>420 \mathrm{~nm})\end{array}$ & $\begin{array}{l}\text { Photoreduction of } \mathrm{CO}_{2} \\
\mathrm{CO}-17.9 \mu \mathrm{mol} \mathrm{g}^{-1} \mathrm{~h}^{-1} \\
\mathrm{O}_{2}-9.8 \mu \mathrm{mol} \mathrm{g}^{-1} \mathrm{~h}^{-1}\end{array}$ & $\begin{array}{c}\text { Reduction in I content in the composite is } \\
\text { unfavourable for the reduction of } \mathrm{CO}_{2} \text {, implying } \\
\mathrm{I}_{3}-\text { intermediate plays an important role in } \\
\text { charge transfer process }\end{array}$ & [185] \\
\hline $\mathrm{g}-\mathrm{C}_{3} \mathrm{~N}_{4} / \mathrm{BiOBr} / \mathrm{Au}$ & $\begin{array}{l}300 \mathrm{~W} \text { Xe lamp } \\
(\lambda>420 \mathrm{~nm})\end{array}$ & $\begin{array}{l}\text { Photoreduction of } \mathrm{CO}_{2} \\
\mathrm{CO}-6.67 \mu \mathrm{mol} \mathrm{g}^{-1} \mathrm{~h}^{-1} \\
\mathrm{CH}_{4}-0.92 \mu \mathrm{mol} \mathrm{g}^{-1} \mathrm{~h}^{-1}\end{array}$ & $\begin{array}{c}\text { The size of Au nanoparticles acted as the } \\
\text { Z-scheme bridge and SPR centre during the } \\
\text { photocatalytic process. }\end{array}$ & [139] \\
\hline $\begin{array}{c}\mathrm{g}-\mathrm{C}_{3} \mathrm{~N}_{4} / \mathrm{BiOCl} \text {-defect } \\
\text { rich }\end{array}$ & $\begin{array}{c}300 \mathrm{~W} \text { Xe lamp } \\
(\lambda>420 \mathrm{~nm})\end{array}$ & $\begin{array}{l}\text { Photoreduction of } \mathrm{CO}_{2} \\
\mathrm{CO}-28.4 \mu \mathrm{mol} \mathrm{g}^{-1} \mathrm{~h}^{-1} \\
\mathrm{CH}_{4}-4.6 \mu \mathrm{mol} \mathrm{g}^{-1} \mathrm{~h}^{-1}\end{array}$ & $\begin{array}{c}\text { Interfacial oxygen vacancies provide a transport } \\
\text { channel for the interfacial carriers, leading to a } \\
\text { built-in electric field promoting enhanced carrier } \\
\text { transfer efficiency. }\end{array}$ & [186] \\
\hline
\end{tabular}




\subsection{Hydrogen Generation}

Hydrogen as a fuel is considered a promising alternative for future energy sustainability owing to its high specific energy and eco-friendly combustion products. The positive $\mathrm{CB}$ position of $\mathrm{BiOX}$ photocatalysts restricts their ability to generate $\mathrm{H}_{2}$, but precise control of their thickness during fabrication and the addition of defects such as oxygen vacancies were reported to simultaneously enhance the visible light absorption and intensity of the self-generated IEF [187-190]. For instance, Ye et al. synthesized black coloured ultrathin $\mathrm{BiOCl}$ nanosheets enriched with oxygen vacancies while glycerol reacted with the oxygen exposed on the (001) surface under hydrothermal conditions. The amount of $\mathrm{H}_{2}$ generated using the black coloured ultrathin $\mathrm{BiOCl}\left(\sim 2.51 \mu \mathrm{mol} \mathrm{h}^{-1}\right)$ under visible light irradiation was about 21 and 15 times higher than bulk $\mathrm{BiOCl}\left(0.12 \mu \mathrm{mol} \mathrm{h}{ }^{-1}\right)$ and $\mathrm{TiO}_{2}(0.16 \mu \mathrm{mol}$ $\mathrm{h}^{-1}$ ), respectively [191].

Li et al. reported the growth of $\mathrm{BiOCl}$ crystal with 18 facets, 24 vertices and 40 edges through a one-pot hydrothermal method for a longer reaction time of $100 \mathrm{~h}$ and, as observed from Figure 7, the amount of photocatalytic $\mathrm{H}_{2}$ generated was 2.1 times greater than that obtained with $\mathrm{BiOCl}$ synthesized with a shorter time span of $10 \mathrm{~h}\left(5.99 \mu \mathrm{mol} \mathrm{g}^{-1} \mathrm{~h}^{-1}\right)$ [192]. Conventionally, the square plates of $\mathrm{BiOCl}$ with exposed $\{001\}$ top facets correspond to the most positive $\mathrm{CB}$ position, while the lateral $\{110\}$ facets form the most negative VB position, facilitating charge separation between in the binary $\{001\} /\{110\}$ facet junction. On the other hand, the eighteen-faceted $\mathrm{BiOCl}$ were composed of $\{001\}$ top facets and unusual $\{102\}$ and $\{112\}$ oblique facets owing to which the $C B$ position was in the order (001) facet $>$ (102) facet $>$ (112) facet, while the VB position order was (001) facet < (102) facet $<(112)$ facet. Therefore, the well-matched $\{001\} /\{102\} /\{112\}$ ternary facet junction in the eighteen-faceted $\mathrm{BiOCl}$ facilitated the efficient cascade charge flow, ensuring enhanced photocatalytic $\mathrm{H}_{2}$ generation. In another report, hierarchical BiOI microspheres synthesized through a microwave-assisted solvothermal method with ethylene glycol and ethanol as solvents were reported to exhibit visible light mediated photocatalytic water splitting to generate $\mathrm{H}_{2}$ with maximum $\left(1316.9 \mu \mathrm{mol} \mathrm{g}^{-1}\right)$ at $\mathrm{pH} 7$ with a dosage of $0.2 \mathrm{gL}^{-1}$. The narrow bandgap of $\mathrm{BiOI}(2.04 \mathrm{eV})$ microspheres, the surprisingly sufficient overpotential due to negative $\mathrm{CB}$ position and the higher separation of the photogenerated charges aided $\mathrm{H}_{2}$ generation [193]. Bai et al. synthesized non-stoichiometric $\mathrm{Bi}_{4} \mathrm{O}_{5} \mathrm{X}_{2}$ $\left(\mathrm{X}=\mathrm{Br}\right.$, I) nanosheets through the molecular precursor method, which generated $\mathrm{H}_{2}$ under a $300 \mathrm{~W}$ lamp emitting simulated solar light irradiation [67]. Using $10 \%$ methanol as the sacrificial agent, the amount of $\mathrm{H}_{2}$ generated with $40 \mathrm{mg}$ of $\mathrm{Bi}_{4} \mathrm{O}_{5} \mathrm{Br}_{2}$ and $\mathrm{Bi}_{4} \mathrm{O}_{5} \mathrm{I}_{2}$ was 4.21 and $2.79 \mu \mathrm{mol} \mathrm{g}^{-1} \mathrm{~h}^{-1}$, respectively. The enhanced photocatalytic performance of $\mathrm{Bi}_{4} \mathrm{O}_{5} \mathrm{Br}_{2}$ nanosheets with a quantum efficiency of $0.93 \%$ at $420 \mathrm{~nm}$ in comparison to $\mathrm{Bi}_{4} \mathrm{O}_{5} \mathrm{I}_{2}$ (just $0.52 \%$ ) was attributed to the greater separation of photogenerated charge carriers. $\mathrm{Bi}_{24} \mathrm{O}_{31} \mathrm{Br}_{10}$ nanoplates synthesized through the chemical precipitation method generated $\mathrm{H}_{2}$ by the photocatalytic reduction of water at a rate of $3.3 \mu \mathrm{mol} \mathrm{h}^{-1}$ with $50 \mathrm{mg}$ catalyst loading, while pristine $\mathrm{BiOBr}$ and $\mathrm{Bi}_{2} \mathrm{O}_{3}$ displayed no activity. The uplifting of the $\mathrm{CB}$ of $\mathrm{Bi}_{24} \mathrm{O}_{31} \mathrm{Br}_{10}$ due to the presence of $\mathrm{Bi} 6 \mathrm{p}$ and $\mathrm{Br} 4 \mathrm{~s}$ orbitals fulfilled the electric potential requirements for splitting water to $\mathrm{H}_{2}$ in comparison to pristine $\mathrm{BiOBr}$ and $\mathrm{Bi}_{2} \mathrm{O}_{3}$ with positive $\mathrm{CB}$ positions [68]. Di et al. reported the synthesis of a defect-rich single-unit-cell of $\mathrm{Bi}_{3} \mathrm{O}_{4} \mathrm{Br}$ with a thickness of $\sim 1.7 \mathrm{~nm}$ that displayed superior photocatalytic $\mathrm{H}_{2}$ generation of up to $380 \mu \mathrm{mol} \mathrm{g}^{-1} \mathrm{~h}^{-1}$, which was $\sim 2$ and 4.9 times greater than defect-deficient $\mathrm{Bi}_{3} \mathrm{O}_{4} \mathrm{Br}$ and bulk $\mathrm{Bi}_{3} \mathrm{O}_{4} \mathrm{Br}$, respectively [97]. The enhanced photocatalytic activity of defect-rich single-unit-cell $\mathrm{Bi}_{3} \mathrm{O}_{4} \mathrm{Br}$ was immensely facilitated by the generation of oxygen defects due to bismuth vacancy in addition to their atomically thin architecture that favourably tuned the electronic band structure. In another study, a bilayer junction formed by the selectively assembly of metallic phase enriched $\mathrm{MoS}_{2}$ and oxygen-deficient $\mathrm{Bi}_{12} \mathrm{O}_{17} \mathrm{Cl}_{2}$ monolayers exhibited photocatalytic $\mathrm{H}_{2}$ evolution at a rate of $33 \mathrm{mmol} \mathrm{h}^{-1} \mathrm{~g}^{-1}$ under visible light, with a superior quantum efficiency of $36 \%$ at $420 \mathrm{~nm}$ that was superior to the pristine monolayers of $\mathrm{MoS}_{2}$ and $\mathrm{Bi}_{\mathrm{x}} \mathrm{O}_{\mathrm{y}} \mathrm{X}_{\mathrm{z}}$-based systems [194]. The enhanced performance of the bilayer $\mathrm{MoS}_{2} / \mathrm{Bi}_{12} \mathrm{O}_{17} \mathrm{Cl}_{2}$ junction can be attributed to the enhanced charge separation 
in oxygen-deficient $\mathrm{Bi}_{12} \mathrm{O}_{17} \mathrm{Cl}_{2}$ monolayers ensured by the IEF and the collective role of both IEF and Bi-S bonds for pushing the electrons to catalyse the $\mathrm{H}_{2}$ evolution. However, surprisingly, to date no reports have been found on photocatalytic $\mathrm{H}_{2}$ generation using a $\mathrm{BiOX} / \mathrm{Bi}_{\mathrm{x}} \mathrm{O}_{\mathrm{y}} \mathrm{X}_{\mathrm{z}}-\mathrm{g}-\mathrm{C}_{3} \mathrm{~N}_{4}$ heterojunction photocatalyst, given the fact that pristine $\mathrm{g}-\mathrm{C}_{3} \mathrm{~N}_{4}$ is an excellent $\mathrm{H}_{2}$ evolution photocatalyst that has been studied extensively.
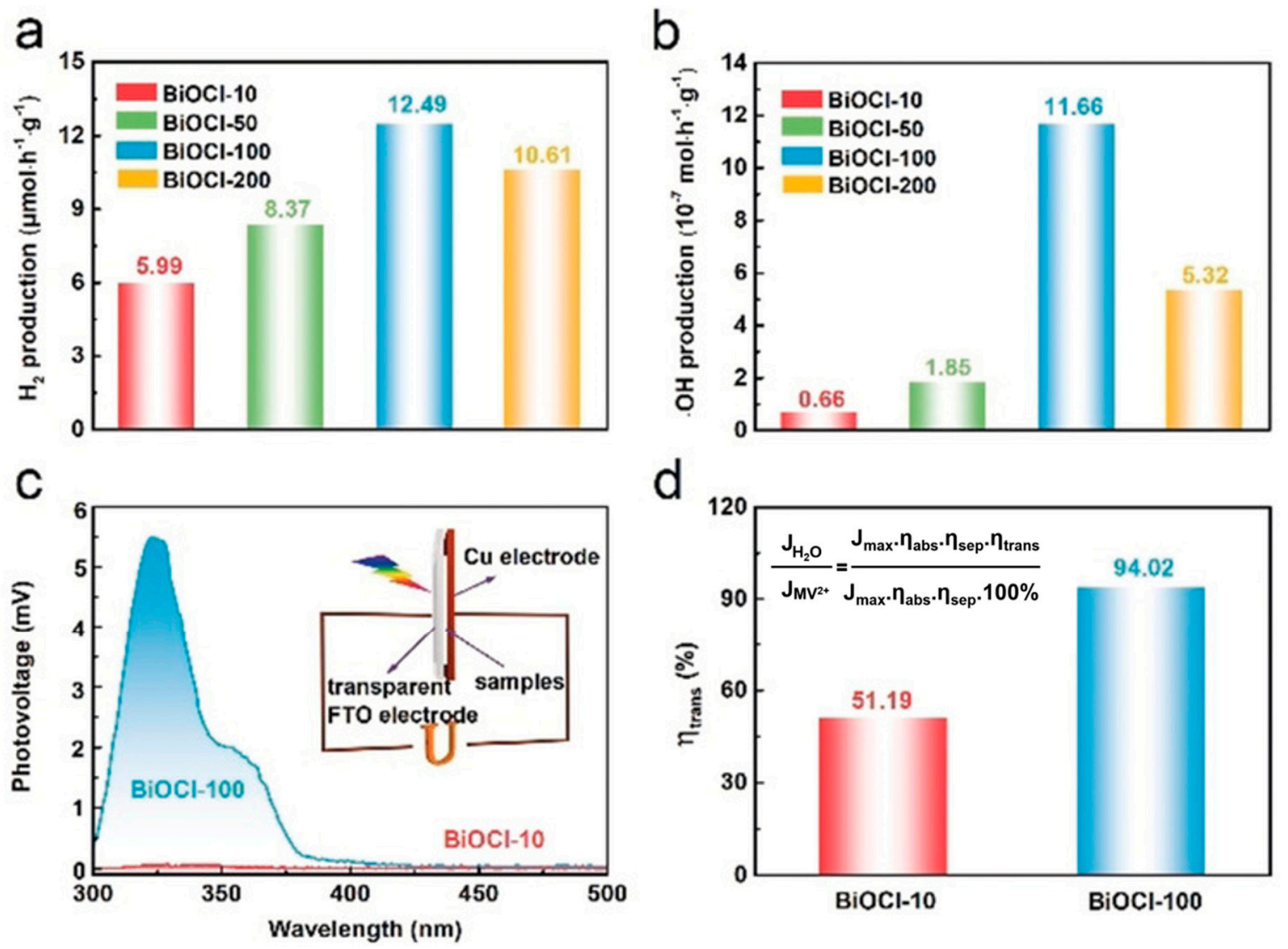

Figure 7. Rate constants for generation of (a) $\mathrm{H}_{2}$ and (b) hydroxyl ions over different BiOCl photocatalysts under simulated solar light irradiation. (c) Surface photovoltage spectra of BiOCl synthesized with time span of $10 \mathrm{~h} \mathrm{(BiOCl-10)} \mathrm{and} 100 \mathrm{~h}$ (BiOCl-100) indicating the degree of charge separation. (d) Surface charge transfer efficiency of BiOCl-10 and BiOCl-100. Reprinted from Ref. [192] with permission from John Wiley \& Sons.

\subsection{Oxygen Evolution}

In addition to $\mathrm{H}_{2}$ generation, sunlight-driven photocatalytic water splitting allows the generation of oxygen $\left(\mathrm{O}_{2}\right)$. However, the water oxidation for $\mathrm{O}_{2}$ evolution is more difficult due to the multistep transfer of four $\mathrm{h}^{+}$in comparison to the transfer of two $\mathrm{e}^{-}$for $\mathrm{H}_{2}$ generation. The oxygen evolution reaction demands the accumulation of cationic $\mathrm{h}^{+}$on the surface (i.e., surface-trapped holes), which is absolutely essential to be utilized for the reduction of adsorbed water via $\mathrm{H}_{2} \mathrm{O}_{\mathrm{ad}}+2 \mathrm{~h}^{+} \rightarrow{ }^{1} / 2 \mathrm{O}_{2}+2 \mathrm{H}^{+}$[195]. The basic requirement for photocatalytic $\mathrm{O}_{2}$ evolution is ensuring that the $\mathrm{VB}$ edge of the photocatalyst is located at a more positive position than the oxidation potential of $\mathrm{H}_{2} \mathrm{O}(1.23 \mathrm{~V}$ vs. normal hydrogen electrode at $\mathrm{pH}=0$ ). Further, a significant overpotential is required for overcoming the activation energies in the charge-transfer process between the photocatalyst and water molecules. Due to the stringent demands, only very few materials are capable of directly oxidizing water into $\mathrm{O}_{2}$ under light irradiation. Di et al. reported the fabrication of atomically thin defect-rich $\mathrm{BiOCl}$ nanosheets through the hydrothermal approach by treating pre-synthesized $\mathrm{BiOCl}$ nanosheets in ethylene glycol and studied their performance towards the photooxidation of $\mathrm{H}_{2} \mathrm{O}$ [196]. The amount of $\mathrm{O}_{2}$ generated with defect-rich $\mathrm{BiOCl}$ nanosheets $\left(56.85 \mu \mathrm{mol} \mathrm{g}{ }^{-1} \mathrm{~h}^{-1}\right)$ was nearly 3 and 8 times greater than that gen- 
erated with defect-free $\mathrm{BiOCl}$ nanosheets and bulk $\mathrm{BiOCl}$. The enhanced performance of defect-rich $\mathrm{BiOCl}$ in the photooxidation of water can be attributed to the synergetic effect of an atomically thin thickness of $\sim 2 \mathrm{~nm}$, defects on $\mathrm{BiOCl}$ basal planes shortening the migration distance of holes for promoting charge separation and hole utilization, and the presence of abundant coordination-unsaturated active atoms. In another study, Ag and $\mathrm{PdO}_{\mathrm{x}}$ nanocubes selectively deposited on the (001) and (110) facets of $\mathrm{BiOCl}$ nanoplates formed a ternary hybrid $\mathrm{Ag}-\mathrm{BiOCl}-\mathrm{PdO}_{\mathbf{x}}$ photocatalyst that was employed in the photocatalytic $\mathrm{O}_{2}$ evolution under visible light with $\mathrm{NaIO}_{3}$ as the electron sacrificial agent [197]. Interestingly, $\mathrm{Ag}-(110) \mathrm{BiOCl}(110)-\mathrm{PdO}_{x}$ exhibited a highest average $\mathrm{O}_{2}$ rate of $68.2 \mu \mathrm{mol}$ $\mathrm{g}^{-1} \mathrm{~h}^{-1}$, which was almost 5.9, 1.9 and 1.6 times higher than Ag-(001)BiOCl(001)-PdO $\mathrm{Ag}-(001) \mathrm{BiOCl}(110)-\mathrm{PdO}_{\mathrm{x}}$ and $\mathrm{Ag}-(110) \mathrm{BiOCl}(001)-\mathrm{PdO}_{\mathrm{x}}$, respectively. The schematic in Figure 8 illustrates the reasons for the enhanced photocatalytic $\mathrm{O}_{2}$ generation, which can be attributed to stronger electronic coupling at the $\mathrm{BiOCl}(110)$-based interfaces as a result of the thinner contact barrier between $\mathrm{Ag}$ and $\mathrm{PdO}_{\mathrm{x}}$ and the shortest average hole diffusion distance realized by $\mathrm{Ag}$ and $\mathrm{PdO}_{\mathrm{x}}$ on the $\mathrm{BiOCl}(110)$ plane.

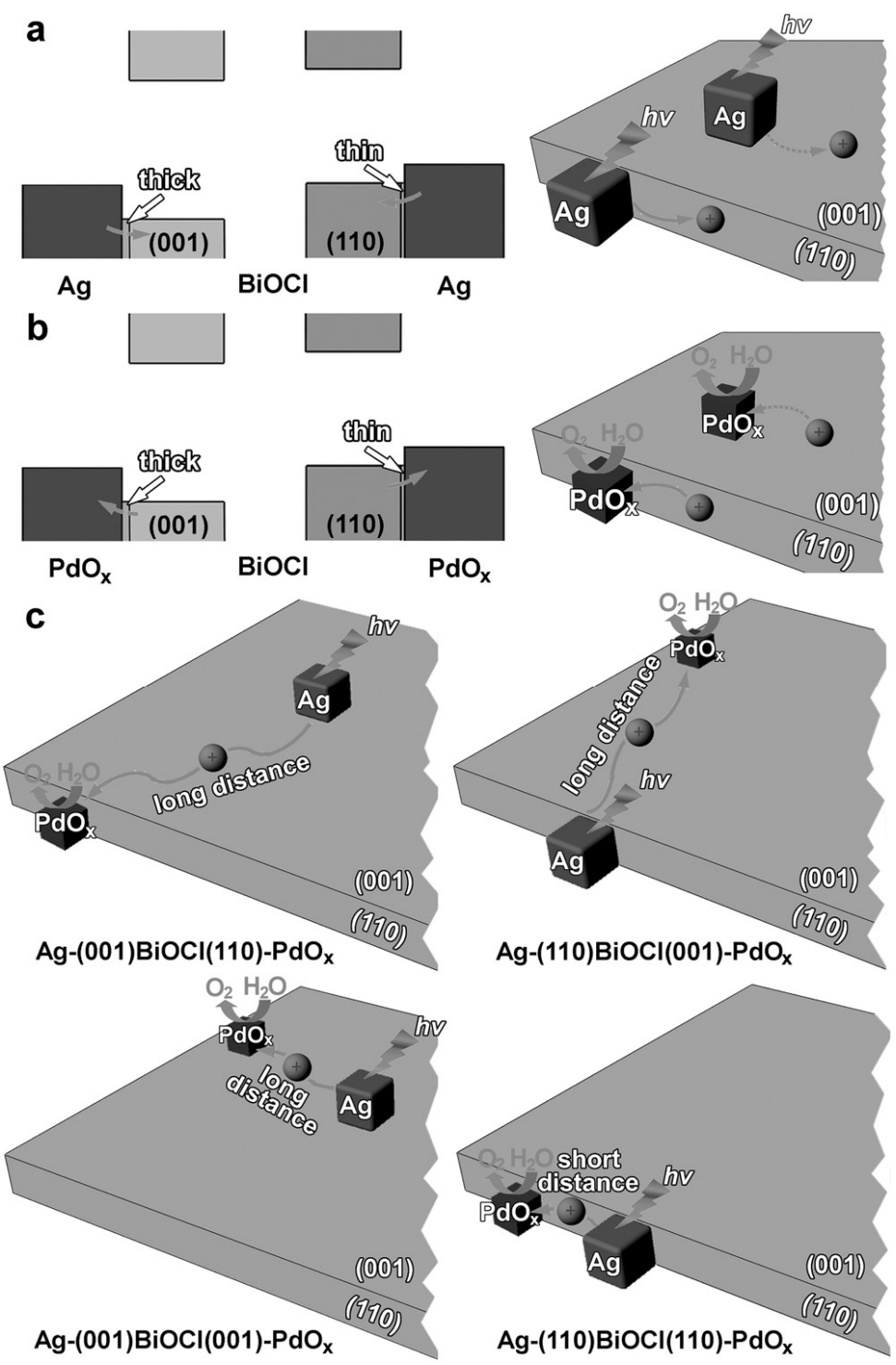

Figure 8. Schematic depicting the facet-dependent interfacial hole transfer ability as a result of the difference in thickness of the contact barrier layer on (a) $\mathrm{Ag}-\mathrm{BiOCl}$ and (b) $\mathrm{BiOCl}-\mathrm{PdO}_{\mathrm{x}}$ interfaces. (c) Schematic representation of the different average diffusion distances of hole in different Ag-BiOCl$\mathrm{PdO}_{\mathrm{x}}$ photocatalysts. Reprinted from Ref. [197] with permission from John Wiley \& Sons. 
Cui et al. reported the solvothermal synthesis of $\mathrm{BiOCl}$ nanosheets with abundant oxygen vacancies using ethylene glycol as the solvent and studied their photooxidation ability [198]. The rate of $\mathrm{O}_{2}$ evolved under visible light with oxygen vacancy-rich $\mathrm{BiOCl}$ nanosheets in the presence of $\mathrm{AgNO}_{3}$ as the electron acceptor was $1.72 \mathrm{mmol} \mathrm{g}{ }^{-1}$ after $5 \mathrm{~h}$, which was 3.3 times higher than oxygen vacancy-poor $\mathrm{BiOCl}$ nanosheets despite the fact that their surface area was almost identical. Abundant oxygen vacancies in $\mathrm{BiOCl}$ nanosheets were reported to create many electron donor levels and allowed the excitation of electrons, which subsequently formed holes in the $\mathrm{VB}$ for the $\mathrm{O}_{2}$ evolution reaction. Similarly, Ji et al. reported the synthesis of oxygen vacancy-rich and oxygen vacancy-less $\mathrm{Bi}_{7} \mathrm{O}_{9} \mathrm{I}_{3}$ microspheres through the ionic liquid assisted solvothermal method and studied their performance in the photocatalytic $\mathrm{O}_{2}$ evolution. As expected, the $\mathrm{O}_{2}$ evolution rate of oxygen vacancy-rich $\mathrm{Bi}_{7} \mathrm{O}_{9} \mathrm{I}_{3}$ microspheres at $199.2 \mu \mathrm{mol} \mathrm{g}^{-1} \mathrm{~h}^{-1}$ was $\sim 1.5$ times greater than that of oxygen vacancy-less $\mathrm{Bi}_{7} \mathrm{O}_{9} \mathrm{I}_{3}$ microspheres, despite the fact that their surface area was comparable [199]. In another study, $\mathrm{Bi}_{3} \mathrm{O}_{4} \mathrm{Br}$ nanorings were synthesized through the solvothermal method using cetyltrimethylammonium bromide and polyvinyl pyrrolidone as surfactants, and their performance was assessed through photocatalytic $\mathrm{O}_{2}$ evolution.

Interestingly, $\mathrm{Bi}_{3} \mathrm{O}_{4} \mathrm{Br}$ exhibited $\mathrm{O}_{2}$ efficient oxygen evolution at a rate of $72.54 \mu \mathrm{mol}$ $\mathrm{g}^{-1} \mathrm{~h}^{-1}$ that was attributed primarily to its single-crystalline nature, (001) facets exposure, ring structure, appropriate light response range and band potential, which facilitated the migration of charge carriers [200]. Ning et al. constructed a 2D-2D heterostructure photocatalyst by coupling $\mathrm{Bi}_{3} \mathrm{O}_{4} \mathrm{Cl}$ and $\mathrm{BiOCl}$ nanosheets through alkaline chemical etching and solvent exfoliation for $\mathrm{O}_{2}$ evolution under visible light [201]. The rate of $\mathrm{O}_{2}$ evolved with ultrathin $\mathrm{Bi}_{3} \mathrm{O}_{4} \mathrm{Cl} / \mathrm{BiOCl}$ in the presence of $\mathrm{FeCl}_{3}$ as the electron scavenger reached $58.6 \mu \mathrm{mol} \mathrm{g}{ }^{-1} \mathrm{~h}^{-1}$, which was about 3 times higher than that of nanocrystal $\mathrm{Bi}_{3} \mathrm{O}_{4} \mathrm{Cl} / \mathrm{BiOCl}$. Electron spin resonance spectroscopy detected ${ }^{\bullet} \mathrm{O}_{2}{ }^{-}$as the primary active species, which strongly suggested the mechanism of charge transfer during the photocatalytic oxidation reaction to be the $\mathrm{Z}$-scheme heterojunction. In the $\mathrm{Bi}_{3} \mathrm{O}_{4} \mathrm{Cl} / \mathrm{BiOCl} \mathrm{Z}$-scheme heterojunction, photogenerated electron-hole pairs generated by the built-in electric field under visible light irradiation enabled the rapid transfer of photogenerated electrons to the $\{001\}-\mathrm{BiOCl}$ facets that were partly trapped by $\mathrm{Fe}^{3+}$, while the holes gathered on the $\{001\}-\mathrm{Bi}_{3} \mathrm{O}_{4} \mathrm{Cl}$ facets accommodated plenty of active sites for the photocatalytic $\mathrm{O}_{2}$ evolution [201]. Though g- $\mathrm{C}_{3} \mathrm{~N}_{4}$ has been extensively studied for its ability to oxidize water under light irradiation [202-204], it is unfortunate that no work on photocatalytic water oxidation has been carried out by designing suitable $\mathrm{BiOX} / \mathrm{Bi}_{\mathrm{x}} \mathrm{O}_{\mathrm{y}} \mathrm{X}_{\mathrm{z}}-\mathrm{g}-\mathrm{C}_{3} \mathrm{~N}_{4}$ heterojunction photocatalysts. However, there is enough scope for constructing efficient heterojunction photocatalysts using $\mathrm{BiOX} / \mathrm{Bi}_{\mathrm{x}} \mathrm{O}_{\mathrm{y}} \mathrm{X}_{\mathrm{z}}$ with exposed facets and functionalized $\mathrm{g}-\mathrm{C}_{3} \mathrm{~N}_{4}$ that could achieve enhanced quantum efficiencies.

\subsection{Nitrogen Reduction}

The photoreduction of nitrogen $\left(\mathrm{N}_{2}\right)$ to produce ammonia $\left(\mathrm{NH}_{3}\right)$, commonly referred to as nitrogen fixation, is a green alternative to the standard Haber-Bosch process, which consumes large amounts of fossil fuels and releases $\mathrm{CO}_{2}$ into the atmosphere. $\mathrm{Li}$ et al. reported the solvothermal synthesis of $\{001\}$ facet exposed $\mathrm{BiOBr}$ nanosheets with and without oxygen vacancies for studying their photocatalytic performance in reducing $\mathrm{N}_{2}$ under visible light irradiation with water as the solvent and proton source. Interestingly, \{001\}-BiOBr without oxygen vacancies did not exhibit photocatalytic activity, while $\{001\}-\mathrm{BiOBr}$ with oxygen vacancies generated a significant amount of $\mathrm{NH}_{3}$ at rate of 104.2 and $223.3 \mu \mathrm{mol} \mathrm{g}^{-1} \mathrm{~h}^{-1}$ under visible light and UV-vis light irradiation, respectively, with an external quantum efficiency of $0.23 \%$ at $420 \mathrm{~nm}$ [205]. $\mathrm{N}_{2}$ was adsorbed on the oxygen vacancies by combining with the two nearest Bi atoms in the sublayer to form a terminal end-on bound structure, and the reduction capacity of $\mathrm{N}_{2}$ over $\{001\}-\mathrm{BiOBr}$ was directly dependent on the amount of oxygen vacancies as they acted as catalytic centres capable of adsorbing and activating $\mathrm{N}_{2}$ by inhibiting electron-hole recombination 
and promoting the interfacial charge transfer. Similarly, the Zhang group also studied photocatalytic $\mathrm{N}_{2}$ fixation using oxygen vacancy-rich $\mathrm{BiOCl}$ nanosheets with $\{001\}$ and $\{010\}$ exposed facets and, interestingly, it was found that the rate of $\mathrm{NH}_{3}$ generation with $\{010\}$-BiOCl $\left(0.95 \mu \mathrm{mol} \mathrm{g}^{-1} \mathrm{~h}^{-1}\right)$ was only half of $\{001\}$-BiOCl $\left(1.89 \mu \mathrm{mol} \mathrm{g}^{-1} \mathrm{~h}^{-1}\right)$, but after $30 \mathrm{~min}$ the rate of $\mathrm{NH}_{3}$ generation with $\{010\}-\mathrm{BiOCl}$ at $2.29 \mu \mathrm{mol} \mathrm{g}^{-1} \mathrm{~h}^{-1}$ was 1.21 times greater than $\{001\}$-BiOCl. The reason for the slower rate of $\mathrm{NH}_{3}$ generation during the initial 30 min was attributed to the different chemistry of $\mathrm{N}_{2}$ fixation on $\{001\}$ and $\{010\}$ facets, while the enhanced $\mathrm{NH}_{3}$ generation was attributed to the more stable side-on bridging of $\mathrm{N}_{2}$ by combining with the two nearest $\mathrm{Bi}$ atoms in the outer layer and the nearest $\mathrm{Bi}$ atom in the sublayer on the (010) surface [206]. Bai et al. synthesized bismuthrich $\mathrm{Bi}_{5} \mathrm{O}_{7} \mathrm{I}$ with $\{001\}$ and $\{100\}$ exposed facets through the solvothermal treatment of molecular precursors in glycerol, and studied their photocatalytic activity for $\mathrm{N}_{2}$ fixation. The $\mathrm{NH}_{3}$ generation rate using $\{001\}-\mathrm{Bi}_{5} \mathrm{O}_{7} \mathrm{I}\left(111.5 \mu \mathrm{mol} \mathrm{g}{ }^{-1} \mathrm{~h}^{-1}\right)$ was $\sim 2.3$ times greater than $\{100\}-\mathrm{Bi}_{5} \mathrm{O}_{7} \mathrm{I}\left(47.6 \mu \mathrm{mol} \mathrm{g}{ }^{-1} \mathrm{~h}^{-1}\right)$, and the apparent quantum efficiency was $5.1 \%$ at $365 \mathrm{~nm}$. Band structure studies through VB X-ray photoelectron spectroscopy revealed the more negative $\mathrm{CB}$ position of $\{001\}-\mathrm{Bi}_{5} \mathrm{O}_{7} \mathrm{I}$ nanosheets that enhanced their reduction power, while the photocurrent response and electrochemical impedance spectroscopy results indicated their enhanced separation of photogenerated charge carriers and lower resistance for electron-transfer. Therefore, it was concluded that the enhanced photocatalytic $\mathrm{N}_{2}$ fixation in Bi-rich $\mathrm{Bi}_{\mathrm{x}} \mathrm{O}_{\mathrm{y}} \mathrm{X}_{\mathrm{z}}$ was due to the facet effect in comparison to $\mathrm{BiOX}$, wherein oxygen vacancies play a dominant role [207]. Another study on Bi-rich $\mathrm{Bi}_{\mathrm{x}} \mathrm{O}_{\mathrm{y}} \mathrm{X}_{\mathrm{z}}$ reported by Wang et al. demonstrated that engineering oxygen vacancies into $\mathrm{Bi}_{5} \mathrm{O}_{7} \mathrm{Br}$ nanotubes with a uniform diameter of $\sim 5 \mathrm{~nm}$ could generate $\mathrm{NH}_{3}$ up to $1.38 \mathrm{mmol} \mathrm{h}^{-1}$ $\mathrm{g}^{-1}$ under visible light with pure water without any organic scavengers or cocatalysts with an apparent quantum efficiency of over $2.3 \%$ at $420 \mathrm{~nm}$ [83]. Interestingly, the $\mathrm{Bi}_{5} \mathrm{O}_{7} \mathrm{Br}$ nanotube dispersion in water exhibited a colour change from light yellow to dark grey under light irradiation that induced oxygen vacancies by seizing $\mathrm{O}$ atoms from water. In addition to the more negative $\mathrm{CB}$ position, the enhanced chemisorption of $\mathrm{N}_{2}$ on the oxygen vacancy sites due to the large surface area of $\mathrm{Bi}_{5} \mathrm{O}_{7} \mathrm{Br}$ nanotubes $\left(96.56 \mathrm{~m}^{2} \mathrm{~g}^{-1}\right)$, forming a bond with Bi-metal (sideward transition metal), enabled it to donate electrons from its bonding orbitals and accept electrons to its antibonding n-orbitals, which gradually wakened the N-N triple bond due to electron exchange and led to the enhanced generation of $\mathrm{NH}_{3}$. Zhang et al. reported photocatalytic $\mathrm{N}_{2}$ fixation by simultaneously introducing oxygen vacancy and doping $\mathrm{Fe}$ into $\mathrm{BiOCl}$ nanosheets that generated $\mathrm{NH}_{3}$ at a rate of $1.02 \mathrm{mmol} \mathrm{g}^{-1} \mathrm{~h}^{-1}$ under light irradiation using a $300 \mathrm{~W}$ Xe lamp [208]. The mechanism of $\mathrm{N}_{2}$ fixation was similar to the report on $\mathrm{Bi}_{5} \mathrm{O}_{7} \mathrm{Br}$ nanotubes, and the dispersion of Fe-doped $\mathrm{BiOCl}$ also exhibited a colour change from white to dark grey under light irradiation for the generation of oxygen vacancies. Typically, the $\mathrm{N}_{2}$ fixation involved four main steps, viz., (i) the generation of oxygen vacancies on the catalyst surface during light irradiation, (ii) the chemisorption of $\mathrm{N}_{2}$ on the catalyst surface activated by oxygen vacancies, (iii) the injection of photogenerated electrons into the orbitals of activated $\mathrm{N}_{2}$ for their reduction, and (iv) the refilling of oxygen vacancies by adjacent $\mathrm{O}$ atoms from $\mathrm{H}_{2} \mathrm{O}$ or $\mathrm{O}_{2}$. Similarly, Fe-doped $\mathrm{BiOBr}$ microspheres composed of nanosheets were synthesized through the solvothermal method with polyethylene glycol for the photocatalytic conversion of $\mathrm{N}_{2}$ to $\mathrm{NH}_{3}$ at a rate of $382.68 \mu \mathrm{mol}^{-1} \mathrm{~g}^{-1} \mathrm{~h}^{-1}$, which was eight times greater than pristine $\operatorname{BiOBr}\left(51.6 \mu \mathrm{mol}^{-1} \mathrm{~g}^{-1} \mathrm{~h}^{-1}\right)$, under visible light radiation obtained from a $300 \mathrm{~W}$ xenon lamp equipped with a $420 \mathrm{~nm}$ cutoff filter [209]. The charge density map of Fe-doped $\mathrm{BiOBr}$ nanosheets shown in Figure 9a indicated that Fe withdrew electrons from nearby atoms to form electron-rich $\mathrm{Fe}(\mathrm{II})$ that injected localized electrons to the п $\mathrm{N}-\mathrm{N}$ antibonding orbital of the adsorbed $\mathrm{N}_{2}$ via electron donation for obtaining enhanced $\mathrm{NH}_{3}$ generation, as observed from Figure 9b. Further, the more negative CB position of Fe-doped $\mathrm{BiOBr}$ nanosheets (Figure 9c) in comparison to pristine $\mathrm{BiOBr}$ and enhanced visible light absorption demonstrated the vital role played by Fe atoms. Similar to defect-rich nanostructures of $\mathrm{BiOX}$ and $\mathrm{Bi}_{\mathrm{x}} \mathrm{O}_{\mathrm{y}} \mathrm{X}_{z}$, defect-rich $\mathrm{g}-\mathrm{C}_{3} \mathrm{~N}_{4}$ has demonstrated excellent performance in the 
photocatalytic $\mathrm{N}_{2}$ fixation under visible light [210,211]. However, to date no work has been reported on photocatalytic $\mathrm{N}_{2}$ fixation with suitable $\mathrm{BiOX} / \mathrm{Bi}_{\mathrm{x}} \mathrm{O}_{\mathrm{y}} \mathrm{X}-\mathrm{g}-\mathrm{C}_{3} \mathrm{~N}_{4}$ heterojunction photocatalysts, which allows room for significant research to be conducted in this direction.

(a)
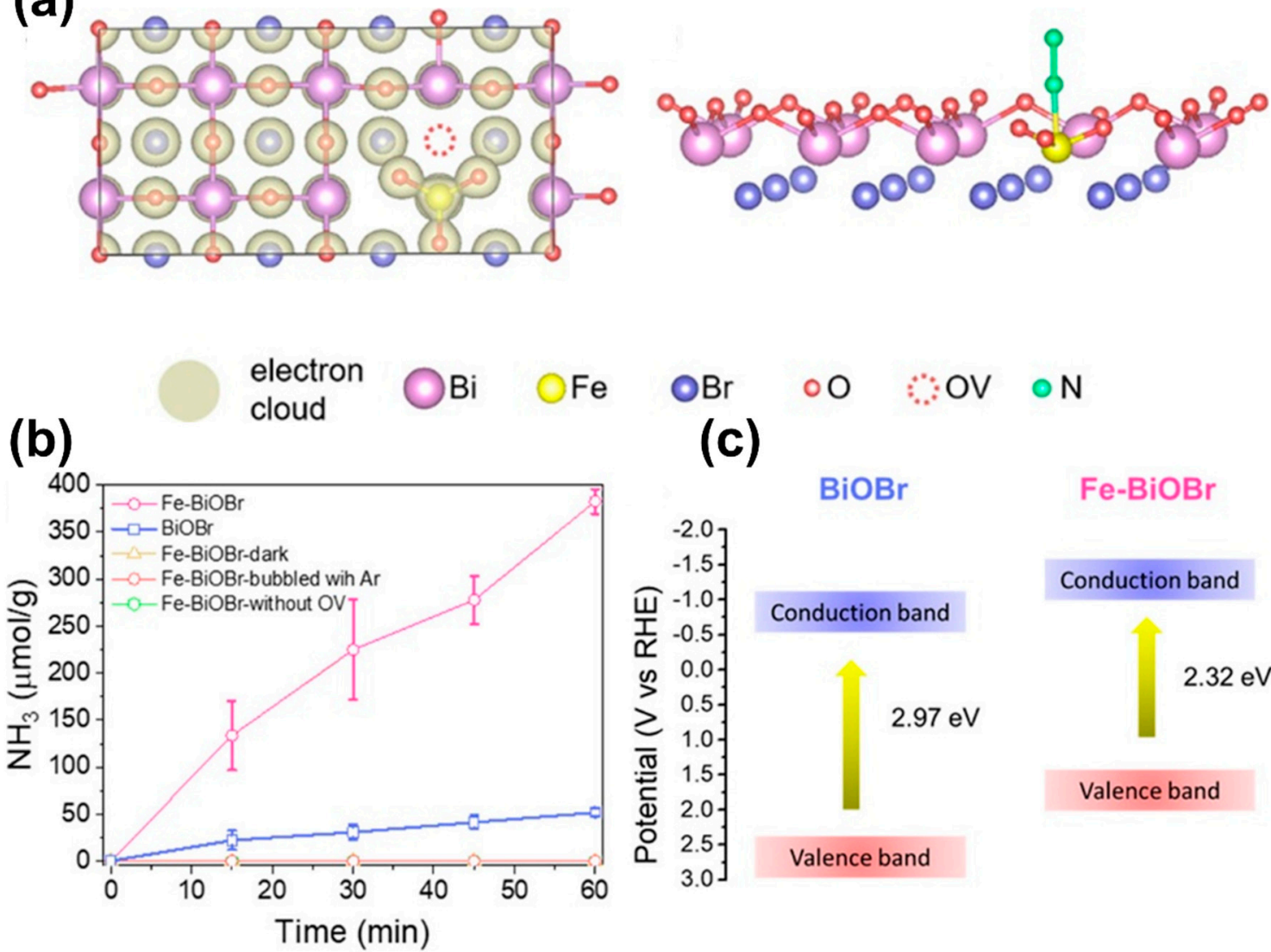

$\mathrm{BiOBr}$

Conduction band

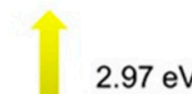

$2.97 \mathrm{eV}$

Valence band
$\mathrm{Fe}-\mathrm{BiOBr}$

Conduction band

$2.32 \mathrm{eV}$

Valence band

Figure 9. (a) Charge density map of Fe-doped BiOBr nanosheets and the schematic of $\mathrm{N}_{2}$ binding to the oxygen vacancy connected $\mathrm{Fe}$ atom in $\mathrm{Fe}-\mathrm{BiOBr}$, (b) plot depicting the photocatalytic $\mathrm{NH}_{3}$ generation using Fe-doped $\mathrm{BiOBr}$ nanosheets and (c) band structure of $\mathrm{BiOBr}$ and Fe-doped $\mathrm{BiOBr}$ nanosheets. Reprinted from Ref. [209] with permission from American Chemical Society.

\subsection{Organic Synthesis}

Semiconductor-based photocatalysis to achieve highly efficient organic reaction has gained significant research attention. The oxidation of alcohols to their corresponding aldehydes is the major area of organic synthesis. Xiao et al. hydrothermally synthesized nanobelt-like structures of $\mathrm{Bi}_{12} \mathrm{O}_{17} \mathrm{Cl}_{2}$ and evaluated their performance towards the photocatalytic oxidation of benzyl alcohol in acetonitrile to benzaldehyde under visible light at $50{ }^{\circ} \mathrm{C}$. The bandgap energy of the $\mathrm{Bi}_{12} \mathrm{O}_{17} \mathrm{Cl}_{2}$ photocatalyst was found to be $2.43 \mathrm{eV}$, and the conversion rate to benzaldehyde was $44 \%$ under oxygen atmosphere via direct hole oxidation. When $\mathrm{Bi}_{12} \mathrm{O}_{17} \mathrm{Cl}_{2}$ nanobelts were subjected to visible light irradiation, the $\mathrm{e}^{-}$ excited to the CB would be trapped by electrophilic $\mathrm{O}_{2}$, while the $\mathrm{h}^{+}$in the VB reacted with alkoxide anions to form carbon radicals through deprotonation, and, subsequently, benzaldehyde was formed by the reaction of these carbon radicals with $\mathrm{h}^{+}$[212]. Han et al. reported the synthesis of $\mathrm{BiOBr}$ photocatalysts with three different exposed facets, viz., $\{001\},\{010\}$ and $\{110\}$ for the selective aerobic photooxidation of benzylamine in acetonitrile solution to N-benzylidenebenzylamine at room temperature and atmospheric air as the oxidizing agent. Although $\mathrm{BiOCl}$ and $\mathrm{BiOI}$ were found to exhibit almost $100 \%$ selectivity for the photooxidation of benzylamine, only $\mathrm{BiOBr}$ exhibited $100 \%$ conversion and selectivity. Results indicated that the orientation of the exposed planes played a significant role as BiOBr-\{001\} exhibited the highest activity based on unit surface area. However, 
solvothermally synthesized BiOBr-\{110\} microspheres achieved $100 \%$ selectivity and conversion efficiency in the oxidation of benzylamine due to their high surface area [213]. $\mathrm{BiOBr}$ nanoplates with (001) exposed facets synthesized through the modified hydrothermal approach were treated in $\mathrm{O}_{2}$ and inert atmosphere for fabricating defect-free and defect-rich BiOBr. Interestingly, the defect-rich $\mathrm{BiOBr}$ nanoplates exhibited high efficiency and selectivity for the oxidation of benzylamine to $\mathrm{N}$-benzylidenebenzylamine, while the yield of corresponding imine was much lower with defect-free BiOBr. Photoluminescence spectroscopy and photoelectrochemical studies confirmed that oxygen-vacancy mediated exciton dissociation resulted in promoted charge-carrier generation in the system that led to a selective oxidative-coupling reaction through ${ }^{\bullet} \mathrm{O}_{2}{ }^{-}$generation [214]. Similarly, $\mathrm{BiOCl}$ colloidal ultrathin nanosheets with hydrophobic surface properties fabricated with abundant oxygen vacancies by the hydrolysis of $\mathrm{BiCl}_{3}$ in octadecylene solution enabled them to display superior photocatalytic activity for the aerobic oxidation of secondary amines to corresponding imines under visible light irradiation [215]. $\mathrm{Bi}_{24} \mathrm{O}_{31} \mathrm{Br}_{10}(\mathrm{OH})_{\delta}$ microspheres containing porous nanosheet substructures with a surface area of $45 \mathrm{~m}^{2} \mathrm{~g}^{-1}$ and abundant active lattice oxygen sites were reported to exhibit the selective photooxidation of various alcohols in air under visible light irradiation [216]. Mott-Schottky analysis suggested the thermodynamically feasible band structure of $\mathrm{Bi}_{24} \mathrm{O}_{31} \mathrm{Br}_{10}(\mathrm{OH})_{\delta}$, while its loose and porous architecture allowed the easy diffusion of bulky alcohols for accessing the abundant active surface sites. Therefore, a remarkably high quantum efficiency of $71 \%$ was achieved under visible light irradiation for isopropanol oxidation. A BiOBr $/ \mathrm{g}-\mathrm{C}_{3} \mathrm{~N}_{4}$ heterojunction photocatalyst synthesized through a two-step combustion-coprecipitation method was reported to exhibit excellent photooxidation of benzylamine to N-benzylidenebenzylamine with a conversion rate of $94 \%$ and a yield of $82 \%$ within $4 \mathrm{~h}$ of visible light irradiation obtained from white LED under atmospheric air [217]. The enhanced performance of the $\mathrm{BiOBr} / \mathrm{g}-\mathrm{C}_{3} \mathrm{~N}_{4}$ photocatalyst was ascribed to the improved charge transfer and separation driven by its apt band structure. Interestingly, bezylamine oxidation happened under both aerobic and anaerobic conditions driven by the ${ }^{\circ} \mathrm{O}_{2}{ }^{-}$radicals (produced by the reaction of $\mathrm{CB} \mathrm{e}^{-}$) with amine cations and the reaction of $\mathrm{VB} \mathrm{h}{ }^{+}$with nitrogen-centred radicals, respectively, to form $\mathrm{N}$ - benzylidenebenzylamine.

\section{Strategies for Improving the Performance of BiOX/Bi $\mathrm{O}_{\mathrm{y}} \mathrm{X}_{\mathrm{z}}-\mathrm{g}-\mathrm{C}_{3} \mathrm{~N}_{4}$ Heterojunction Photocatalysts}

The photocatalytic performance of $\mathrm{BiOX} / \mathrm{Bi}_{x} \mathrm{O}_{y} \mathrm{X}_{z}$ nanomaterials has received substantial research interest owing to their suitable band structure for absorbing sunlight to start the photocatalytic reaction. Unfortunately, their practical applications are still confined by a few drawbacks, including a mismatch between the band edge position and light harvesting, ineffective charge separation and transportation, fewer active sites, and poor selectivity of the desired reaction. In this context, numerous strategies have been developed to engineer the layered structure and overcome the aforementioned drawbacks. The following sections emphasize each strategy accordingly.

\subsection{Microstructure Modulation}

Due to the strong connection between the physical and chemical properties and the microstructure (shape, size, surface area, and dimensionality) of the materials, the rational synthesis of the nano- or microstructure has constantly received great significance from the prospect of both scientific research and industrial applications. Further, the inherent nature of nanoscale materials to exhibit higher surface-to-volume ratio and provide abundant active sites enables the effective separation of the photoinduced carriers, thereby enhancing their photocatalytic efficiency. Table 1 provides the summary of various methods for fabricating $\mathrm{BiOX} / \mathrm{Bi}_{\mathrm{x}} \mathrm{O}_{\mathrm{y}} \mathrm{X}_{\mathrm{z}}-\mathrm{g}-\mathrm{C}_{3} \mathrm{~N}_{4}$ heterojunction photocatalysts which were briefly introduced in Section 4.1. Since many articles have already reviewed the importance of microstructure modulation, our discussions in this section are confined to just a few articles mainly focusing on the fabrication of the heterojunction between $\mathrm{BiOX} / \mathrm{Bi}_{\mathrm{x}} \mathrm{O}_{\mathrm{y}} \mathrm{X}_{\mathrm{z}}$ 
and $g-\mathrm{C}_{3} \mathrm{~N}_{4}$. In addition to the conjunction 2D-2D heterojunction, the embedment of 3D hierarchical structures on 2D structures has also sparked interest owing to the distinctive 3D architecture formed by the self-assembly of 1D and 2D sub-structures.

For example, a $\mathrm{Bi}_{5} \mathrm{O}_{7} \mathrm{I} / \mathrm{g}-\mathrm{C}_{3} \mathrm{~N}_{4}$ heterojunction photocatalyst was synthesized by two different approaches, adopting in situ co-thermolysis [106] and the one-pot ethylene glycol assisted hydrothermal approach [107]. In the in situ co-thermolysis method, BiOI precursor (pre-synthesized through the coprecipitation method) was mixed with melamine and ground with an agate mortar, and the powdered material taken in a crucible was heated in a muffle furnace at $520{ }^{\circ} \mathrm{C}$ for $4 \mathrm{~h}$ for obtaining $\mathrm{Bi}_{5} \mathrm{O}_{7} \mathrm{I} / \mathrm{g}-\mathrm{C}_{3} \mathrm{~N}_{4}$. On the other hand, in the one-pot ethylene glycol assisted hydrothermal approach, a final solution of ethylene glycol made by the dropwise addition of $\mathrm{KI}$ solution to a solution containing $\mathrm{Bi}\left(\mathrm{NO}_{3}\right)_{3} \bullet 5 \mathrm{H}_{2} \mathrm{O}$ with pre-synthesized g- $\mathrm{C}_{3} \mathrm{~N}_{4}$ was treated hydrothermally at $150{ }^{\circ} \mathrm{C}$ for $12 \mathrm{~h}$. Interestingly, the morphology of the final structure of $\mathrm{Bi}_{5} \mathrm{O}_{7} \mathrm{I} / \mathrm{g}-\mathrm{C}_{3} \mathrm{~N}_{4}$ resembled microspheres with nanosheet substructures of the individual components. Additionally, interestingly, the estimated values of the $\mathrm{VB}$ and $\mathrm{CB}$ potentials for $\mathrm{g}-\mathrm{C}_{3} \mathrm{~N}_{4}(1.54 \mathrm{eV}$ and $-1.19 \mathrm{eV})$ and $\mathrm{Bi}_{5} \mathrm{O}_{7} \mathrm{I}(3.17 \mathrm{eV}$ and $0.29 \mathrm{eV})$ were identical. However, the mechanism of charge transfer described for the $\mathrm{Bi}_{5} \mathrm{O}_{7} \mathrm{I} / \mathrm{g}-\mathrm{C}_{3} \mathrm{~N}_{4}$ heterojunction photocatalyst synthesized through in situ co-theromolysis was ascribed to the type-II heterojunction, while the charge transfer mechanism in hydrothermally synthesized $\mathrm{Bi}_{5} \mathrm{O}_{7} \mathrm{I} / \mathrm{g}-\mathrm{C}_{3} \mathrm{~N}_{4}$ was ascribed to the $\mathrm{Z}$-scheme. In another study, $\mathrm{BiOBr} / \mathrm{g}-\mathrm{C}_{3} \mathrm{~N}_{4}$ heterojunction photocatalysts were fabricated by dispersing pre-synthesized $\mathrm{BiOBr}$ nanoflowers enriched with oxygen vacancies synthesized by the solvothermal treatment of precursors $\left(\mathrm{Bi}\left(\mathrm{NO}_{3}\right)_{3} \bullet 5 \mathrm{H}_{2} \mathrm{O}\right.$, polyvinylpyrrolidone and $\mathrm{KBr}$ ) dispersed in mixed solvent (ethylene glycol and water) at $160{ }^{\circ} \mathrm{C}$ for $3 \mathrm{~h}$, in g- $\mathrm{C}_{3} \mathrm{~N}_{4}$ dispersion and stirring at room temperature for $6 \mathrm{~h}$, followed by washing and drying [218].

TEM analysis indicates the layered $\mathrm{g}-\mathrm{C}_{3} \mathrm{~N}_{4}$ structure with ultrathin nanosheets (Figure 10a), the nanoflower-like morphology of $\mathrm{BiOBr}$ enriched with oxygen vacancies (Figure 10c) and their perfect heterojunction, indicating the embedment of the oxygen vacancy enriched nanoflowers on g- $\mathrm{C}_{3} \mathrm{~N}_{4}$ nanosheets (Figure 10d). Comparatively, the morphology of defectfree $\mathrm{BiOBr} / \mathrm{g}-\mathrm{C}_{3} \mathrm{~N}_{4}$ indicates the formation of nanoplates, as observed from Figure $10 \mathrm{~b}$. HRTEM micrographs (Figure 10e,f) of oxygen vacancy enriched $\mathrm{BiOBr} / \mathrm{g}-\mathrm{C}_{3} \mathrm{~N}_{4}$ depict the lattice spacing of $d=0.28$ and $0.352 \mathrm{~nm}$ corresponding to the (102) and (101) crystal planes of the tetragonal phase of $\mathrm{BiOBr}$, respectively. Further, the purity and co-existence of all the elements in $\mathrm{BiOBr} / \mathrm{g}-\mathrm{C}_{3} \mathrm{~N}_{4}$ were confirmed from EDS elemental mapping, as shown in Figure $10 \mathrm{~g}$. The photocatalytic activity of oxygen vacancy enriched $\mathrm{BiOBr} / \mathrm{g}-\mathrm{C}_{3} \mathrm{~N}_{4}$ in the removal of $\mathrm{NO}$ under visible light irradiation at $63 \%$ was $1.8,1.6,1.6$ and 1.5 times greater than pristine g- $\mathrm{C}_{3} \mathrm{~N}_{4}$, pristine oxygen vacancy enriched $\mathrm{BiOBr}$, defect-free $\mathrm{BiOBr} / \mathrm{g}$ $\mathrm{C}_{3} \mathrm{~N}_{4}$ and a physical mixture of $\mathrm{g}-\mathrm{C}_{3} \mathrm{~N}_{4}$ with oxygen vacancy enriched $\mathrm{BiOBr}$. Similarly, photocatalytic $\mathrm{CO}_{2}$ reduction using oxygen vacancy enriched $\mathrm{BiOBr} / \mathrm{g}-\mathrm{C}_{3} \mathrm{~N}_{4}$ generated $\mathrm{CO}$ and $\mathrm{CH}_{4}$ at a rate of 61.8 and $27.1 \mu \mathrm{molh}^{-1} \mathrm{~g}^{-1}$, respectively, which was greater than the control samples. Abundant oxygen vacancies in $\mathrm{BiOBr}$ and the heterojunction with ultrathin $\mathrm{g}_{-} \mathrm{C}_{3} \mathrm{~N}_{4}$ nanosheets were attributed to the enhanced photocatalytic activity, while the ${ }^{\bullet} \mathrm{OH}$ and ${ }^{\bullet} \mathrm{O}_{2}{ }^{-}$radicals were reported to be the main active species involved in the removal of $\mathrm{NO}$ and the reduction of $\mathrm{CO}_{2}$, respectively. 

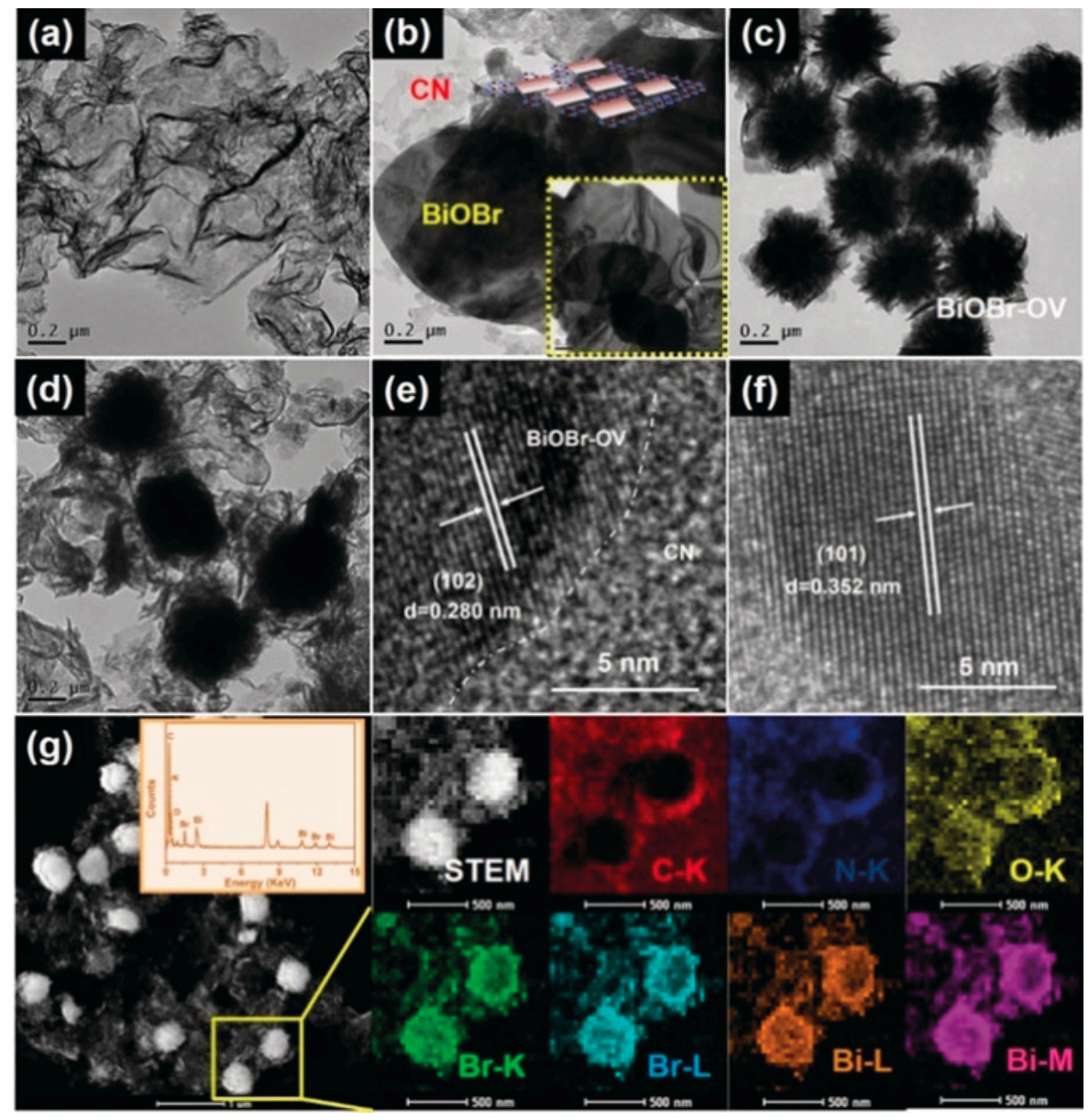

Figure 10. TEM micrographs of (a) pristine $g-\mathrm{C}_{3} \mathrm{~N}_{4}$, (b) defect free- $\mathrm{BiOBr} / \mathrm{g}-\mathrm{C}_{3} \mathrm{~N}_{4}$, (c) pristine $\mathrm{BiOBr}$ enriched with oxygen vacancies, (d) oxygen vacancy enriched $\mathrm{BiOBr} / \mathrm{g}-\mathrm{C}_{3} \mathrm{~N}_{4},(\mathbf{e}, \mathbf{f}) \mathrm{HRTEM}$ micrographs of oxygen vacancy enriched $\mathrm{BiOBr} / \mathrm{g}-\mathrm{C}_{3} \mathrm{~N}_{4}$ depicting the lattice spacing and $(\mathrm{g})$ the corresponding elemental maps of $\mathrm{C}, \mathrm{N}, \mathrm{O}, \mathrm{Br}$ and $\mathrm{Bi}$. Reprinted from Ref. [218] with permission from Wiley-VCH.

\subsection{Facet and Defect Control}

Crystal facets are an important feature of crystalline materials, and different crystal facets have different geometric and electronic structures, exhibiting intrinsic reactivity and surface physical and chemical properties associated with the crystallographic orientation. As a basic feature of crystalline materials, the exposed crystal facets play an important role in photocatalytic efficiency since photocatalysis occurs on the surface of BiOX photocatalysts. $\mathrm{BiOCl}$ nanosheets with tunable $\{001\}$ facet percentages were synthesized by hydrolyzing molecular precursors $\mathrm{Bi}_{n}(\mathrm{Tu})_{x} \mathrm{Cl}_{3 \mathrm{n}}(\mathrm{Tu}=$ thiourea). Exposed $\{001\}$ facets of $\mathrm{BiOCl}$ exhibited high oxygen atom density, and under UV light irradiation, plenty of oxygen vacancy sites were created [219]. These oxygen vacancies formed a defect state near the bottom of the $\mathrm{CB}$ of $\mathrm{BiOCl}$ and played a significant role in capturing the photogenerated electrons for enhancing the photocatalytic activity of $\mathrm{BiOCl}$ due to the improved separation of photogenerated charge carriers. Jiang et al. reported the hydrothermal synthesis of $\mathrm{BiOCl}$ single-crystalline nanosheets with exposed $\{001\}$ facets, which exhibited higher activity for direct semiconductor photoexcitation pollutant degradation under UV light, while the counterpart with exposed $\{010\}$ facets possessed superior activity for indirect dye photosensitization degradation under visible light [220]. Zhao et al. obtained rose-like BiOBr nanostructures with exposed $\{111\}$ facets using sodium dodecyl sulphate as the surfactant, which exhibited better photocatalytic activity than exposed $\{001\}$ facets under both visible light and monochromatic light [221]. Although high-energy facets exhibited higher 
activity than low-energy facets, they are easily eliminated because the fastest crystal growth would occur in the direction perpendicular to the high-energy facet. Therefore, glucose as the capping and structure-directing agent was employed in synthesizing $1 \mathrm{D}$ rod-like $\mathrm{BiOBr}$ with exposed $\{110\}$ facets, and it was revealed that glucose not only suppressed the growth of $\{001\}$ facets of $\mathrm{BiOBr}$ nanosheets but also induced these nanosheets to self-assemble along the [1] orientation, displaying better photocatalytic activity towards the photodegradation of rhodamine B and methyl orange [222]. Defects in the exposed facets of semiconductors can significantly enhance the photocatalytic activity by changing their electronic structures, the recombination efficiency of charge carriers, and surface properties [223]. As a typical defect, oxygen vacancies are reported to enhance the photo-absorption and photocatalytic performance of the photocatalysts. Li et al. reported the fabrication of $\mathrm{BiOBr}$ nanosheets with oxygen vacancies via a hydrothermal-reduction route. Their study revealed that only those oxygen vacancies created on the surface of the photocatalyst could inhibit the charge carrier recombination by trapping the photogenerated electrons, while the bulk oxygen vacancies which can also trap photogenerated charges act as recombination centres, resulting in a decrease in photoactivity [205]. Wang et al. reported the introduction of surface oxygen vacancies over the $\mathrm{BiOBr}$ nanosheets exposed with $\{001\}$ facets by surface modification using polybasic carboxylic acids. These surface oxygen vacancies on $\mathrm{BiOBr}$ intensified the separation efficiency of photogenerated carriers and promoted the dioxygen reduction towards the degradation of MO dye [224]. Further, density functional theory calculations revealed that the presence of oxygen vacancies can ensure the increased density of states at the conduction band edge relative to the $\mathrm{BiOBr}$ atomic layers and bulk counterpart, which helps in enhancing the electron transport pathways.

Additionally, the introduced oxygen vacancies created new defect levels which allowed a narrower bandgap, hence giving the possibility for realizing visible light $\mathrm{CO}_{2}$ reduction. Wu et al. reported that oxygen-deficient $\mathrm{BiOBr}$ atomic layers triggered visiblelight-driven $\mathrm{CO}_{2}$ reduction into $\mathrm{CO}$ with a rate of $87.4 \mu \mathrm{mol} \mathrm{g}^{-1} \mathrm{~h}^{-1}$, which was 20 times and 24 times higher than that of $\mathrm{BiOBr}$ atomic layers and bulk $\mathrm{BiOBr}$. Thus, defect engineering was proved to promote $\mathrm{CO}_{2}$ photoreduction efficiency through fully addressing the poor photo-absorption, sluggish electron-hole separation, and high $\mathrm{CO}_{2}$ activation barrier, giving new possibilities for achieving high performance in solar $\mathrm{CO}_{2}$ reduction [225].

$\mathrm{Li}$ et al. reported the synthesis of a $\mathrm{BiOCl}$ single crystal with eighteen-facets by prolonging the hydrothermal reaction time (10-200 h), which exhibited enhanced $\mathrm{H}_{2}$ generation that was higher than previously reported $\mathrm{BiOCl}$ with a [1] top facet and [121] lateral facets. SEM micrographs of $\mathrm{BiOCl}$ crystals synthesized at different time intervals are represented in Figure 11a-d. Both TEM and HRTEM micrographs confirmed the formation of well-shaped oblique facets at an angle of $\sim 45^{\circ}$ from the top facets, as observed from Figure $11 \mathrm{e}-\mathrm{h}$. The schematic illustration of eighteen-faceted $\mathrm{BiOCl}$ and the $\{001\},\{102\}$ and $\{112\}$ facets in $\mathrm{BiOCl}$ is represented in Figure 11i,j. The well-indexed XRD patterns indicated the formation of a pure phase of $\mathrm{BiOCl}$ (Figure 11k). Therefore, with the help of the ternary facet junction, the electron-hole pairs in the eighteen-faceted $\mathrm{BiOCl}$ single crystal were effectively separated and displayed outstanding photocatalytic activity in the generation of $\mathrm{H}_{2}$ [192]. 


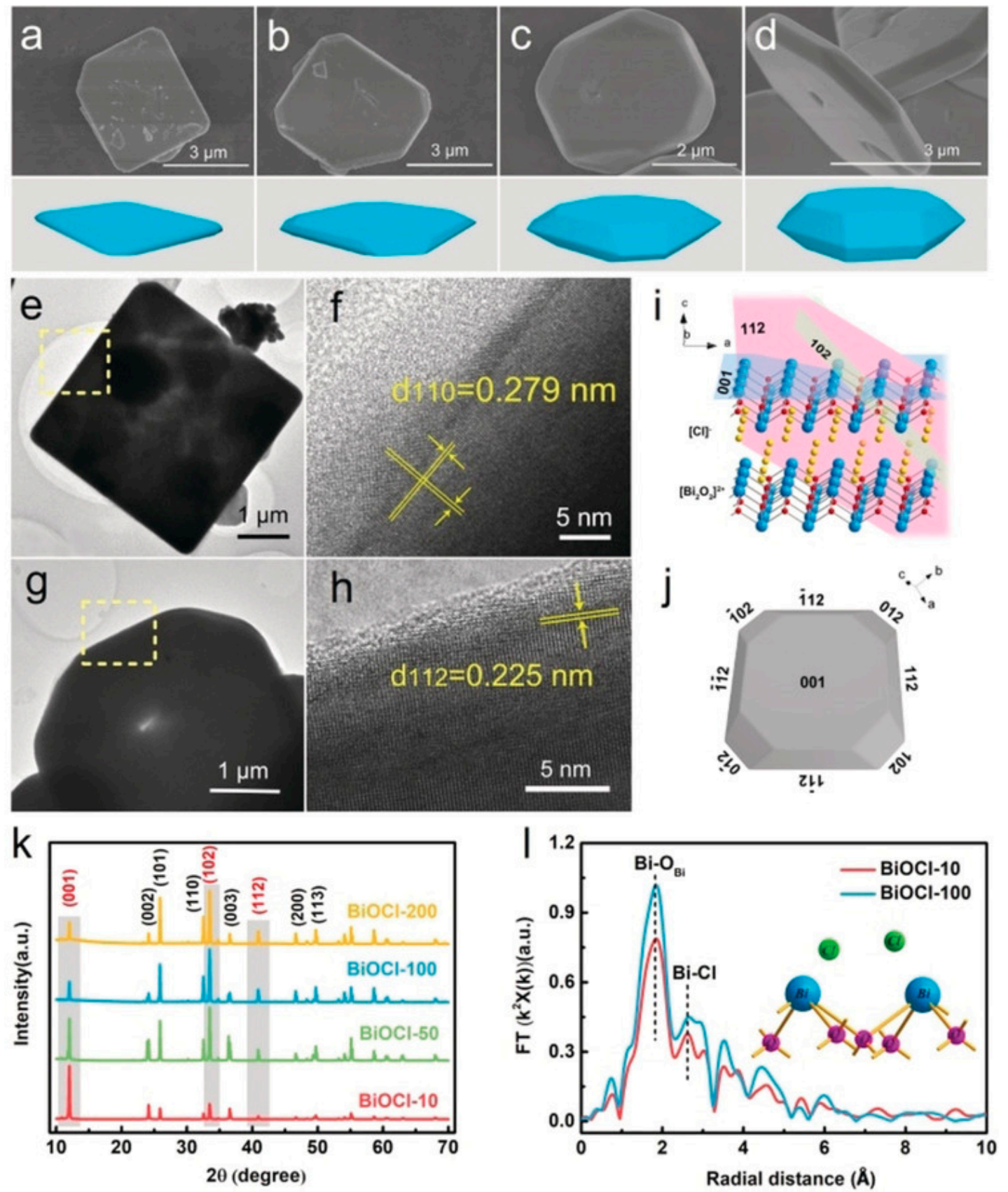

Figure 11. SEM images and the corresponding schematic representation for BiOCl treated at different time intervals (a) $10 \mathrm{~h},(\mathbf{b}) 50 \mathrm{~h}$, (c) $100 \mathrm{~h}$, and (d) $200 \mathrm{~h}$; (e) TEM image and (f) HRTEM image of BiOCl treated for $10 \mathrm{~h}$; (g) spherical aberration correction TEM image and (h) HRTEM image of $\mathrm{BiOCl}$ treated for $100 \mathrm{~h}$; (i) crystal structure and facets of BiOCl; (j) Schematic representation of the different facets of eighteen-faceted $\mathrm{BiOCl}$; (k) XRD patterns of BiOCl series samples; (1) Fourier transformed profiles for Bi coordination environments in normalized Bi L3-edge XAFS spectra of BiOCl treated at 10 and $100 \mathrm{~h}$. Reprinted from Ref. [192] with permission from John Wiley \& Sons.

\subsection{Integration with Noble Metal Nanostructures}

Depositing the noble metal over the surface of the semiconductor surface is an effective approach for modifying the photon harvesting capacity and for increasing the charge carrier separation kinetics. Noble metals coupled with semiconductor photocatalysts could form a high-speed charge-transfer channel for accelerated transport. Further, in many semiconductor systems, the noble metal nanoparticles have been usually used as charge-transfer mediators owing to their excellent electron conductivity, thereby offering a new approach to overcome the limit of traditional heterojunction photocatalysts. Additionally, due to the 
unique phenomenon of surface plasmon resonance (SPR) and its induced local electric field, the noble metal nanoparticles bridged with semiconductor photocatalysts can strengthen the photon absorption range and boost the photoinduced electron transfer $[226,227]$. Jiang et al. coupled Pt nanoparticles as the apt co-catalyst in the ternary Z-scheme photocatalytic system with $\mathrm{BiOI}(5.65 \mathrm{eV})$ and $\mathrm{g}-\mathrm{C}_{3} \mathrm{~N}_{4}(4.52 \mathrm{eV})$, since the work function of $\mathrm{Pt}(5.20 \mathrm{eV})$ was in between that of the individual semiconductors. Interestingly, the efficiency of the $\mathrm{BiOI} / \mathrm{Pt} / \mathrm{g}-\mathrm{C}_{3} \mathrm{~N}_{4}$ system was much higher than that of pristine $\mathrm{g}-\mathrm{C}_{3} \mathrm{~N}_{4}, \mathrm{Pt} / \mathrm{g}-\mathrm{C}_{3} \mathrm{~N}_{4}$, and $\mathrm{BiOI} / \mathrm{g}-\mathrm{C}_{3} \mathrm{~N}_{4}$ in the photodegradation of phenol and tetracycline hydrochloride, which was attributed to the efficient separation and transfer of charge carriers in an unobstructed $\mathrm{Z}$-scheme route. The electric fields in the opposite direction for Pt/BiOI and Pt/g- $\mathrm{C}_{3} \mathrm{~N}_{4}$ interfaces were formed due to the difference in the work function. The higher work function of $\mathrm{Pt}$ in contact with g- $\mathrm{C}_{3} \mathrm{~N}_{4}$ formed a Schottky barrier and forced the transfer of electrons accumulated in the space charge region, resulting in the upward band bending in the $\mathrm{Pt} / \mathrm{g}-\mathrm{C}_{3} \mathrm{~N}_{4}$ interface. Similarly, the depletion layer at $\mathrm{Pt} / \mathrm{BiOI}$ was formed due to the higher work function of $\mathrm{BiOI}$ in comparison to Pt. Under visible light irradiation, the inverse electric field at $\mathrm{Pt} / \mathrm{g}-\mathrm{C}_{3} \mathrm{~N}_{4}$ and $\mathrm{Pt} / \mathrm{BiOI}$ would induce the $\mathrm{e}^{-}$in $\mathrm{BiOI}$ and $\mathrm{h}^{+}$in g- $\mathrm{C}_{3} \mathrm{~N}_{4}$ to combine at the Pt metal. Since this charge transfer process occurred without overcoming the Schottky barrier, it was termed as an unobstructed Z-scheme heterojunction and enabled the photogenerated $\mathrm{e}^{-}$in the $\mathrm{CB}$ of $\mathrm{g}_{-} \mathrm{C}_{3} \mathrm{~N}_{4}$ and $\mathrm{h}^{+}$in the $\mathrm{VB}$ of BiOI to form ${ }^{\bullet} \mathrm{O}_{2}{ }^{-}$and ${ }^{\bullet} \mathrm{OH}$ radicals that efficiently degraded the organic contaminants [136].

\subsection{Carbonaceous Materials Compounding}

Carbonaceous materials, such as graphene, carbon nanotubes (CNTs), carbon quantum dots (CQDs), carbon fibres, multi-walled carbon nanotubes (MWCNTs), carbon spheres, etc., are reported to play a vital role in enhancing the photocatalytic performance of $\mathrm{BiOX} / \mathrm{Bi}_{\mathrm{x}} \mathrm{O}_{\mathrm{y}} \mathrm{X}_{\mathrm{z}}$ nanomaterials $[94,145,147,148]$. Graphene or reduced graphene oxide (rGO) has been considered a good electron collector and charge transport medium in photocatalysis owing to its high conductivity, excellent electron mobility, and large specific surface area. $\mathrm{BiOCl} /$ carbon-based photocatalysts have gained enormous interest due to their enhanced performance, which was attributed to their strong adsorption, excellent light absorption, and rapid transfer of photogenerated charges [228,229]. A BiOCl/CQDs/rGO ternary heterojunction photocatalyst driven by visible light exhibited enhanced ciprofloxacin removal efficiency, which was attributed to the excellent adsorption, enhanced charge separation and charge injection induced by the presence of CQDs and rGO. The photocatalytic efficiency of $\mathrm{BiOCl} / \mathrm{CQDs} / \mathrm{rGO}$ was 3.8 and 10.4 times greater in comparison to $\mathrm{BiOCl} / \mathrm{CQDs}$ and $\mathrm{BiOCl}$, respectively, while the removal efficiency was $\sim 87 \%$ [230]. Yu et al. hydrothermally synthesized 3D BiOBr/rGO heterostructured aerogel using dopamine as both a reducing agent and cross-linker. The rate of the photodegradation of $\mathrm{MO}(80 \%)$ using $3 \mathrm{D}$ $\mathrm{BiOBr} / \mathrm{rGO}$ was much higher compared to $\mathrm{RhB} \mathrm{(50 \% )} \mathrm{and} \mathrm{phenol} \mathrm{(35 \% )} \mathrm{under} 60 \mathrm{~min}$ of visible light irradiation. Strong $\pi-\pi$ interaction through the conjugative aromatic structure was attributed to the highly efficient selective adsorption of anionic MO [231]. Similarly, the addition of $1 \mathrm{wt} \% \mathrm{rGO}$ relative to $\mathrm{BiOBr}$ sheets with exposed $\{001\}$ facets with a core/shell structure exhibited the highest activity for the photodegradation of orange II dye $(97 \%$ in $90 \mathrm{~min}$ ) and the removal of acetaminophen ( $93 \%$ in $105 \mathrm{~min}$ ). The enhanced photocatalytic activity of $(1 \mathrm{wt} \%) \mathrm{rGO} / \mathrm{BiOBr}$ was attributed to increased visible light absorption, effective separation, the transportation of photogenerated charge carriers and the formation of a Schottky barrier at the interface between $\mathrm{BiOBr}$ and $\mathrm{rGO}$, which enabled the transfer of $\mathrm{e}^{-}$from the $\mathrm{CB}$ of $\mathrm{BiOBr}$ to $\mathrm{rGO}$ (due to its higher work function) and the internal electric field at the interface. The capability of $\mathrm{rGO}$ to store and shuttle $\mathrm{e}^{-}$enabled the formation of - $\mathrm{O}_{2}{ }^{-}$radicals by reacting with adsorbed $\mathrm{O}_{2}$ molecules, while allowing the $\mathrm{h}^{+}$to react with $\mathrm{OH}$ to form ${ }^{\bullet} \mathrm{OH}$, the two main species responsible for the oxidation of organic contaminants [232]. Z-scheme heterojunction photocatalysts with solid-state electron mediators bridging two semiconductors were proposed for enhancing the performance through the efficient transport and separation of the photogenerated charge carriers. For instance, a 
2D/2D Z-scheme heterojunction was constructed between $\mathrm{BiOBr}$ and g- $\mathrm{C}_{3} \mathrm{~N}_{4}$ using carbon dots as the solid-state electron mediator, and it exhibited enhanced photocatalytic performance in the degradation of ciprofloxacin $(\sim 84 \%$ in $105 \mathrm{~min})$ and tetracycline $(\sim 83 \%$ in $60 \mathrm{~min}$ ) under visible light degradation. Under visible light irradiation, the photogenerated $\mathrm{e}^{-}$in the $\mathrm{CB}$ of $\mathrm{BiOBr}$ with low reduction ability and photogenerated $\mathrm{h}^{+}$in the $\mathrm{VB}$ of g- $\mathrm{C}_{3} \mathrm{~N}_{4}$ with low oxidation ability are transferred to the carbon dots, while the $\mathrm{e}^{-}$and $\mathrm{h}^{+}$ with high reduction and oxidation ability produce ${ }^{\bullet} \mathrm{O}_{2}{ }^{-}$and ${ }^{\bullet} \mathrm{OH}$ active species that react with the organic contaminants for their mineralization into $\mathrm{CO}_{2}$ and $\mathrm{H}_{2} \mathrm{O}$ [145]. Similarly, the unique electron mediating feature of carbon dots coupled with $\mathrm{BiOBr}(20 \mathrm{wt} \%)$ and g- $\mathrm{C}_{3} \mathrm{~N}_{4}$ nanosheets facilitated the improved separation of photogenerated charge carriers for superior performance towards the degradation of organic contaminants (rhodamine $\mathrm{B}$, methylene blue and methyl orange) and the photoreduction of $\mathrm{Cr}(\mathrm{VI})$ to $\mathrm{Cr}(\mathrm{III})$ under visible light, with ${ }^{\bullet} \mathrm{O}_{2}{ }^{-}$and ${ }^{\bullet} \mathrm{OH}$ being the active species [94]. Likewise, rGO was employed as an electron transfer mediator in the heterojunction formed between $\mathrm{BiOBr}(10 \mathrm{wt} \%)$ with protonated $\mathrm{g}-\mathrm{C}_{3} \mathrm{~N}_{4}$ for the photodegradation of tetracycline (59\% mineralized) and $\mathrm{BiOCl}$ with protonated g- $\mathrm{C}_{3} \mathrm{~N}_{4}$ for the photodegradation of tetracycline $(96 \%$ in $180 \mathrm{~min})$ and the selective oxidation of benzyl alcohol (conversation rate $76 \%$ and selectivity $99 \%$ ). In both cases, the mechanism of photocatalysis followed the Z-scheme heterojunction, with ${ }^{\bullet} \mathrm{O}_{2}{ }^{-}$and ${ }^{\bullet} \mathrm{OH}$ being the primary active species $[144,233]$. On the other hand, MWCNTs were also reported to have been employed as electron mediators in the heterojunction between $\mathrm{g}_{-} \mathrm{C}_{3} \mathrm{~N}_{4}$ and $\mathrm{BiOI}(20 \mathrm{wt} \%)$. The heterojunction exhibited improved visible light photocatalytic activity towards the degradation of methylene blue (10 ppm, 70\% in $3 \mathrm{~h}$ ) under visible light $(>420 \mathrm{~nm})$ through the Z-scheme mediated charge transfer [142]. A p-n junction formed by coupling g- $\mathrm{C}_{3} \mathrm{~N}_{4}$ and $\mathrm{BiOBr}$ with $\mathrm{rGO}$ as the conductive support exhibited enhanced photocatalytic activity in the degradation of rhodamine B (10 ppm, $66 \%$ in $60 \mathrm{~min}$ ) under visible light. The $\mathrm{sp}^{2}$-hybridized carbon atoms in graphene capable of storing and shuttling electrons enabled the photogenerated electrons from the $\mathrm{CB}$ of $\mathrm{BiOBr}$ to flow into it and formed a Schottky barrier at the interface for preventing their backflow. Meanwhile, the electrons with high reduction potential and holes with high oxidation potential reacted with dissolved $\mathrm{O}_{2}$ and $\mathrm{OH}^{-}$to form ${ }^{\bullet} \mathrm{O}_{2}{ }^{-}$and ${ }^{\bullet} \mathrm{OH}$ radicals, which were actively involved in the photodegradation of rhodamine $\mathrm{B}$ [146]. In comparison to CNTs, carbon dots and rGO, mussel-inspired biometric carbon material polydopamine, also possessing a conjugated $\Pi$ structure and good electron transport ability, has attracted significant interest owing to its excellent adhesion ability, strong light-harvesting capacity, photoconductivity and biocompatibility. The Z-scheme heterojunction photocatalyst g- $\mathrm{C}_{3} \mathrm{~N}_{4} @$ polydopamine/BiOBr showed high activity in the photocatalytic degradation of sulfamethoxazole under visible light. Polydopamine was reported to promote the efficient separation of the photogenerated charge carriers for ensuring efficient redox capability of the photocatalyst, while the mechanism studied through radical quenching experiments confirmed that the $\mathrm{h}^{+}$and ${ }^{\bullet} \mathrm{O}_{2}{ }^{-}$were the major reactive species for oxidizing sulfamethoxazole [169].

\subsection{Integration of Other Semiconductor Nanostructures}

Single component photocatalysts fail to exhibit higher photocatalytic efficiency due to the rapid recombination of the photogenerated charge carriers. In order to achieve enhanced photocatalytic efficiency, one of the most common strategies is to construct a heterojunction photocatalytic system by coupling two or more semiconductors [234]. Typically, in a heterojunction photocatalytic system, the photogenerated electrons in the $\mathrm{CB}$ of photocatalyst $\mathrm{A}$ migrate to the $\mathrm{CB}$ of photocatalyst $\mathrm{B}$, while the photogenerated holes in the VB of photocatalyst B move to the VB of photocatalyst A, curbing their recombination due to spatial isolation. However, after the charge transfer, the redox ability of the photogenerated charges becomes weakened since the top of the VB potential of photocatalyst $A$ is less positive than that of photocatalyst $B$, and the bottom of the $C B$ potential of photocatalyst B is less negative than that of photocatalyst A. Due to this 
drawback, the heterojunction photocatalytic system (referred to as type-II heterojunction) fails to simultaneously possess high charge-separation efficiency and strong redox ability. Therefore, a Z-scheme photocatalytic process was proposed by carefully studying the natural photosynthesis reaction in plants, which also features the spatial isolation of the photogenerated charges to hinder their recombination. Although the structure of direct Z-scheme photocatalyst is similar to that of a type-II heterojunction photocatalyst, its charge-carrier migration mechanism is different and the pathway resembles the letter " $\mathrm{Z}$ ". During the photocatalytic reaction, the photogenerated electrons in photocatalyst $B$ with lower reduction ability recombine with the photogenerated holes in photocatalyst A with lower oxidation ability. Therefore, the photogenerated electrons in photocatalyst A with high reduction ability and the photogenerated holes in photocatalyst B with high oxidation ability can perform the redox reactions without any hindrance, and the performance of the resulting Z-scheme photocatalytic system can be optimized. Further, the large number of defects aggregated at the contact interface exhibits properties similar to that of conductors with low electrical resistance owing to the fact that energy levels at the interface become quasi-continuous $[235,236]$.

Since 2D nanostructures can offer an apt platform for establishing surface contact with other species, the idea of constructing a heterojunction by the hybridization of two types of 2D photocatalysts is an appropriate strategy for increasing the interface area. Recently, the combination of $g-\mathrm{C}_{3} \mathrm{~N}_{4}$ with $\mathrm{BiOX} / \mathrm{Bi}_{\mathrm{x}} \mathrm{O}_{\mathrm{y}} \mathrm{X}_{\mathrm{z}}$ for the construction of $2 \mathrm{D} / 2 \mathrm{D}$ heterojunction photocatalysts has attracted considerable research attention [237-240]. Liu et al. reported the fabrication of a $\mathrm{BiOBr} / \mathrm{g}-\mathrm{C}_{3} \mathrm{~N}_{4}$ heterojunction through a simple reflux process, and its photocatalytic performance was studied by the degradation of rhodamine $\mathrm{B}$ and bisphenol A under visible light irradiation. The enhanced photocatalytic performance of the heterojunction composite was obviously attributed to the efficient charge generation and separation, while the active species involved during the photodegradation of rhodamine $\mathrm{B}$ and bisphenol A were found to be in the order ${ }^{\bullet} \mathrm{OH}>\mathrm{h}^{+}>{ }^{\bullet} \mathrm{O}_{2}{ }^{-}$. For determining whether the photocatalytic mechanism followed the type-II heterojunction or Z-scheme system, the migration channel of the photogenerated electron-hole pairs was analysed through UV-Vis diffused reflectance spectroscopy and X-ray photoelectron spectroscopy. The results revealed that the values of $\mathrm{CB}$ and $\mathrm{VB}$ potentials of pristine $\mathrm{BiOBr}$ nanoplates were $0.30 \mathrm{eV}$ and $3.07 \mathrm{eV}$, while those of pristine $\mathrm{g}-\mathrm{C}_{3} \mathrm{~N}_{4}$ nanosheets were $-1.12 \mathrm{eV}$ and $1.58 \mathrm{eV}$, respectively. After the hybridization of $\mathrm{g}-\mathrm{C}_{3} \mathrm{~N}_{4}$ with $\mathrm{BiOBr}$, the $\mathrm{VB}$ edge of the $\mathrm{BiOBr} / \mathrm{g}-\mathrm{C}_{3} \mathrm{~N}_{4}$ heterojunction was found to be shifted to $1.32 \mathrm{eV}$ due to the alignment of the Fermi levels at the interface. Under visible light irradiation, the photogenerated electrons moved from the $\mathrm{CB}$ of $\mathrm{g}-\mathrm{C}_{3} \mathrm{~N}_{4}$ to that of $\mathrm{BiOBr}$, while the photogenerated holes moved from the $\mathrm{VB}$ of $\mathrm{BiOBr}$ to that of $\mathrm{g}-\mathrm{C}_{3} \mathrm{~N}_{4}$ across the intimate well-aligned band structure due to the potential difference. As observed from Figure 12a, if $\mathrm{BiOBr} / \mathrm{g}-\mathrm{C}_{3} \mathrm{~N}_{4}$ had formed a type-II heterojunction, the formation of ${ }^{\bullet} \mathrm{O}_{2}{ }^{-}$and ${ }^{\bullet} \mathrm{OH}$ would not have been possible due to the insufficient reduction and oxidation potential of $\mathrm{BiOBr}$ and $\mathrm{g}-\mathrm{C}_{3} \mathrm{~N}_{4}$. Therefore, the Z-scheme photocatalytic system was found to be constructed as shown in Figure $12 b$, wherein the electrons accumulated in the $\mathrm{CB}$ of $\mathrm{g}-\mathrm{C}_{3} \mathrm{~N}_{4}(-1.12 \mathrm{eV}$ vs. NHE) reacted with oxygen molecules to form ${ }^{\bullet} \mathrm{O}_{2}{ }^{-}$, and the holes in the $\mathrm{VB}$ of $\mathrm{BiOBr}(3.07 \mathrm{eV}$ vs. NHE) reacted with $\mathrm{OH}^{-}$to generate ${ }^{\bullet} \mathrm{OH}$ radicals [123].

A direct solid-state Z-scheme heterojunction photocatalyst was constructed by coupling nanosheets of $\mathrm{BiOI}$ and $\mathrm{g}-\mathrm{C}_{3} \mathrm{~N}_{4}$ for the photodegradation of toxic microcystin-LR under visible light irradiation. The rate constant of the best performing $g-\mathrm{C}_{3} \mathrm{~N}_{4} / \mathrm{BiOI}$ heterojunction photocatalyst $\left(0.4357 \mathrm{~h}^{-1}\right)$ was three and five times greater than pristine $\mathrm{BiOI}$ and g- $\mathrm{C}_{3} \mathrm{~N}_{4}$, respectively, and radical scavenger studies revealed that ${ }^{\circ} \mathrm{O}_{2}{ }^{-}$played the major role in the degradation of microcystin-LR. If BiOI/g- $\mathrm{C}_{3} \mathrm{~N}_{4}$ had formed a type-II heterojunction, the formation of ${ }^{\bullet} \mathrm{OH}$ and ${ }^{\bullet} \mathrm{O}_{2}{ }^{-}$would not have been possible due to the insufficient reduction and oxidation potential of $\mathrm{BiOI}$ and $\mathrm{g}-\mathrm{C}_{3} \mathrm{~N}_{4}$. Therefore, the direct $\mathrm{Z}$ scheme charge transfer mechanism occurred, wherein the photogenerated charges formed in the $\mathrm{CB}$ of $\mathrm{g}-\mathrm{C}_{3} \mathrm{~N}_{4}$ and the $\mathrm{VB}$ of $\mathrm{BiOI}$ with high reduction and oxidation ability reacted 
with $\mathrm{O}_{2}$ and $\mathrm{OH}^{-}$to generate ${ }^{\bullet} \mathrm{O}_{2}{ }^{-}$and ${ }^{\bullet} \mathrm{OH}$, respectively, while the photogenerated charges with low reduction and oxidation ability recombined at the interface [111]. Tian et al. reported the fabrication of two p-n junction photocatalysts by coupling different facets of BiOI with $\mathrm{g}-\mathrm{C}_{3} \mathrm{~N}_{4}$ through a simple precipitation method, and studied their feasibility for the photodegradation of various organic contaminants such as 2,4-dichlorophenol, bisphenol A, rhodamine B and tetracycline hydrochloride [112]. Typically, the $\{001\}$ facet of $\mathrm{BiOI}$ was coupled with the $\{002\}$ facet of $\mathrm{g}-\mathrm{C}_{3} \mathrm{~N}_{4}$ to form a (001)-BiOI/(002)-g- $\mathrm{C}_{3} \mathrm{~N}_{4}$ photocatalyst through parallel assembly, and (110)-BiOI/ $(002)^{+}-\mathrm{g}-\mathrm{C}_{3} \mathrm{~N}_{4}$ was fabricated by the vertical assembly of the $\{110\}$ facet of $\mathrm{BiOI}$ on the positively charged $\{002\}$ facet of $\mathrm{g}-\mathrm{C}_{3} \mathrm{~N}_{4}$. The results indicated that the top-top facets coupled (001)-BiOI/(002)-g- $\mathrm{C}_{3} \mathrm{~N}_{4}$ photocatalyst exhibited more than four times enhanced performance in the photodegradation of bisphenol $\mathrm{A}$ and tetracycline hydrochloride in comparison to the laterally assembled (110)-BiOI-(002) ${ }^{+}$-g- $\mathrm{C}_{3} \mathrm{~N}_{4}$.

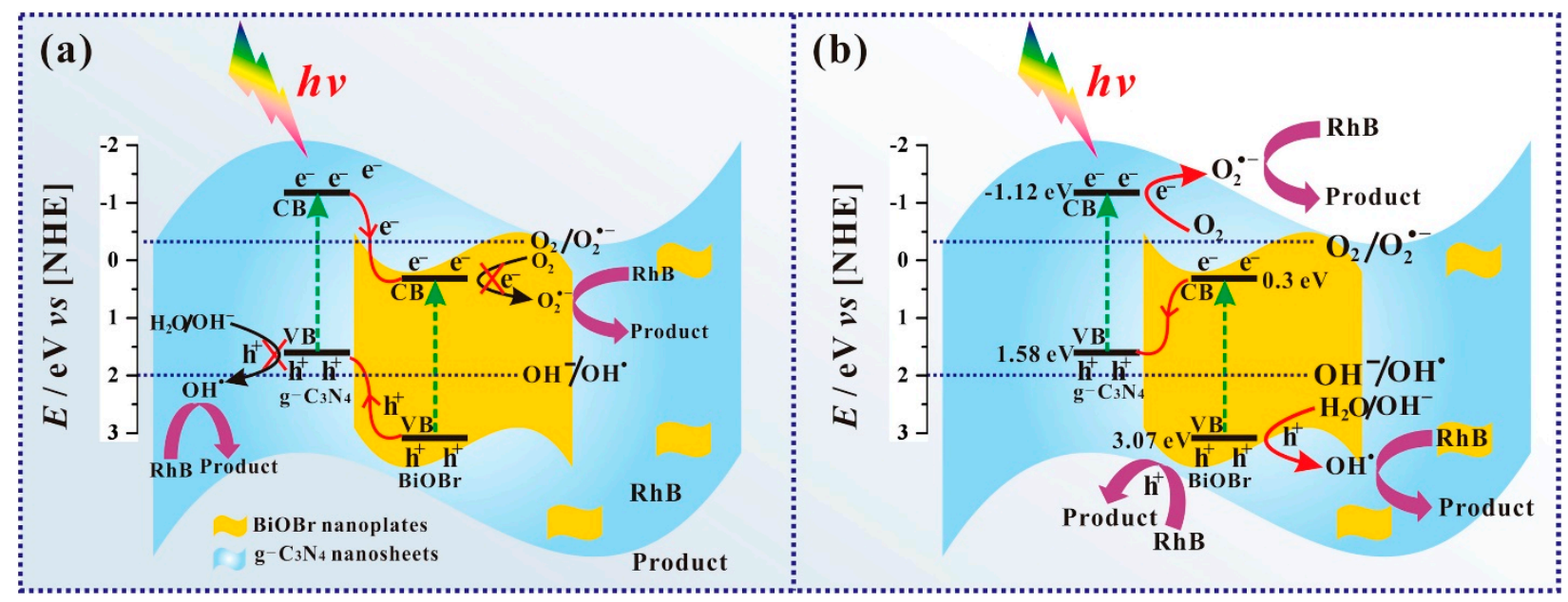

Figure 12. Schematic depicting (a) type-II heterojunction and (b) Z-scheme system, the two possible photocatalytic charge transfer processes in the heterojunction between $\mathrm{BiOBr}$ nanoplates and $\mathrm{g}-\mathrm{C}_{3} \mathrm{~N}_{4}$ nanosheets under visible light irradiation. Reprinted from Ref. [123] with permission from Elsevier.

As shown in Figure 13a, the fermi energy level of BiOI as a p-type semiconductor is located close to the $\mathrm{VB}$, while in the case of $\mathrm{g}-\mathrm{C}_{3} \mathrm{~N}_{4}$, it is located close to the $\mathrm{CB}$ and the energy levels of both the semiconductors achieve an equilibrium to form a (001)$\mathrm{BiOI} /(002)-\mathrm{g}-\mathrm{C}_{3} \mathrm{~N}_{4} \mathrm{p}-\mathrm{n}$ heterojunction photocatalyst. The formation of the p-n junction effectively separates the photogenerated electron-hole pairs in both the heterojunction photocatalysts, but the transfer rate of the photogenerated electrons was found to be distinctly different in the two heterojunctions. In the case of the (001)-BiOI/(002)-g- $\mathrm{C}_{3} \mathrm{~N}_{4} \mathrm{p}$ $n$ heterojunction photocatalyst shown in Figure 13b, the IEF of BiOI along the [1] direction lying perpendicular to the $\mathrm{g}-\mathrm{C}_{3} \mathrm{~N}_{4}$ nanosheets results in the rapid enrichment of the electrons on $\mathrm{g}-\mathrm{C}_{3} \mathrm{~N}_{4}$ that benefitted the subsequent reduction reactions for the generation of ${ }^{1} \mathrm{O}_{2}$ and ${ }^{\bullet} \mathrm{O}_{2}{ }^{-}$radical species. On the other hand, in the case of the (110)-BiOI/(002) ${ }^{+}$-g$\mathrm{C}_{3} \mathrm{~N}_{4}$ p-n heterojunction photocatalyst shown in Figure 13c, the charge transfer direction was parallel, and due to the long diffusion distance, some of the electrons recombined with the holes, leading to inefficient charge transfer in the heterojunction. 


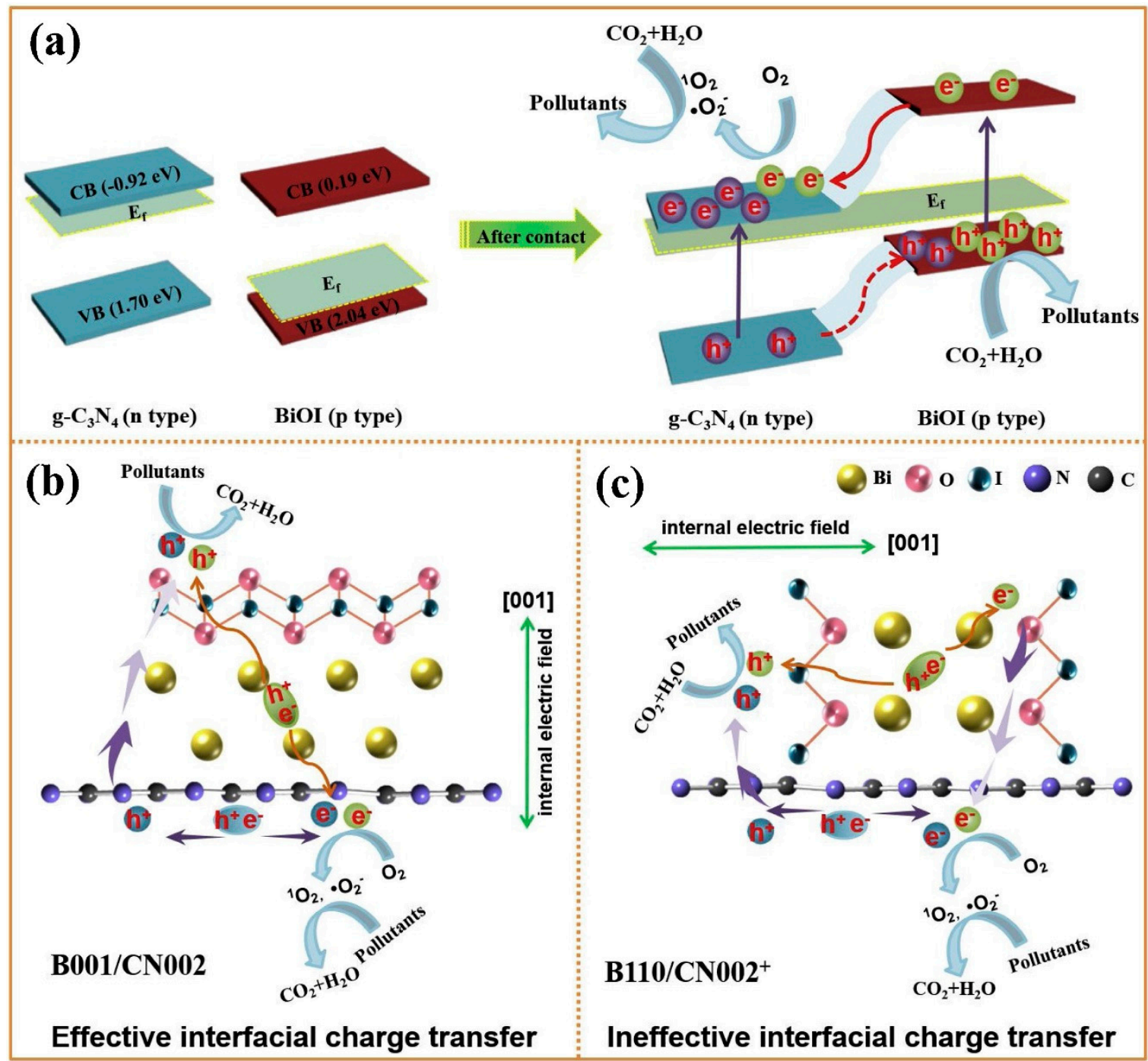

Figure 13. (a) Schematic depicting the formation of p-n junction and the proposed charge separation process in a heterojunction formed by coupling the top-top facets of $\mathrm{BiOI}$ and $\mathrm{g}-\mathrm{C}_{3} \mathrm{~N}_{4}$, i.e., (001)-BiOI/(002)-g- $\mathrm{C}_{3} \mathrm{~N}_{4}$ under visible light irradiation. Schematic depicting the proposed photodegradation mechanism over (b) (001)-BiOI/(002)-g- $\mathrm{C}_{3} \mathrm{~N}_{4}$ and (c) (110)-BiOI/ (002) ${ }^{+}-\mathrm{g}-\mathrm{C}_{3} \mathrm{~N}_{4}$ (positively charged $\mathrm{g}-\mathrm{C}_{3} \mathrm{~N}_{4}$ ). Reprinted from Ref. [112] with permission from Elsevier.

The development of ternary or multicomponent heterojunction systems was considered owing to the possibility of enhancing the charge separation and transfer ability and extending the scope of light absorption as compared to binary systems. For instance, the AgBr@g- $\mathrm{C}_{3} \mathrm{~N}_{4} / \mathrm{BiOBr}$ ternary composite was fabricated through hydrothermal processing and an in situ ion-exchange route for dispersing $\mathrm{AgBr}$ nanoparticles between the g- $\mathrm{C}_{3} \mathrm{~N}_{4} / \mathrm{BiOBr}(2 \mathrm{D} / 2 \mathrm{D})$ heterojunction. Interestingly, $\mathrm{BiOBr}$ played a central role between g- $\mathrm{C}_{3} \mathrm{~N}_{4}$ and $\mathrm{AgBr}$ for providing a high-speed charge transfer channel and isolating the photogenerated charge carriers, resulting in high photocatalytic efficiency for the degradation of rhodamine B (10 ppm, 94\% in $30 \mathrm{~min})$ and tetracycline hydrochloride (10 ppm, 78\% in 2 h) [160]. The ternary heterojunction between $\mathrm{Bi}_{24} \mathrm{O}_{31} \mathrm{Cl}_{10}, \mathrm{MoS}_{2}$ and g- $\mathrm{C}_{3} \mathrm{~N}_{4}$ was synthesized through the impregnation-calcination method. The higher photocatalytic efficiency of the g- $\mathrm{C}_{3} \mathrm{~N}_{4} / \mathrm{MoS}_{2} / \mathrm{Bi}_{24} \mathrm{O}_{31} \mathrm{Cl}_{10}$ ternary heterojunction photocatalyst in the degradation of tetracycline hydrochloride ( $20 \mathrm{ppm}, \sim 97 \%$ in $50 \mathrm{~min}$ ) under visible light was attributed to its enhanced light absorption capacity, the rapid separation of the photogenerated charges and the strong redox ability. The mechanism of charge transfer was reported to follow a dual Z-scheme pathway as depicted in Figure 14, wherein the photogenerated $\mathrm{e}^{-}$with less reduction ability from the $\mathrm{CB}$ of $\mathrm{g}-\mathrm{C}_{3} \mathrm{~N}_{4}$ and $\mathrm{MoS}_{2}$ jump to the $\mathrm{VB}$ of $\mathrm{Bi}_{24} \mathrm{O}_{31} \mathrm{Cl}_{10}$ for 
recombining with the holes, while the $\mathrm{e}^{-}$with strong reduction ability and $\mathrm{h}^{+}$with strong oxidation stability are spared. Scavenger studies and electron spin resonance spectroscopy confirmed the involvement of ${ }^{\bullet} \mathrm{O}_{2}{ }^{-}$and ${ }^{\bullet} \mathrm{OH}$ radical species, which also confirms the transfer of the photogenerated charge carriers through the dual Z-scheme pathway for ensuring enhanced photocatalytic efficiency [155].
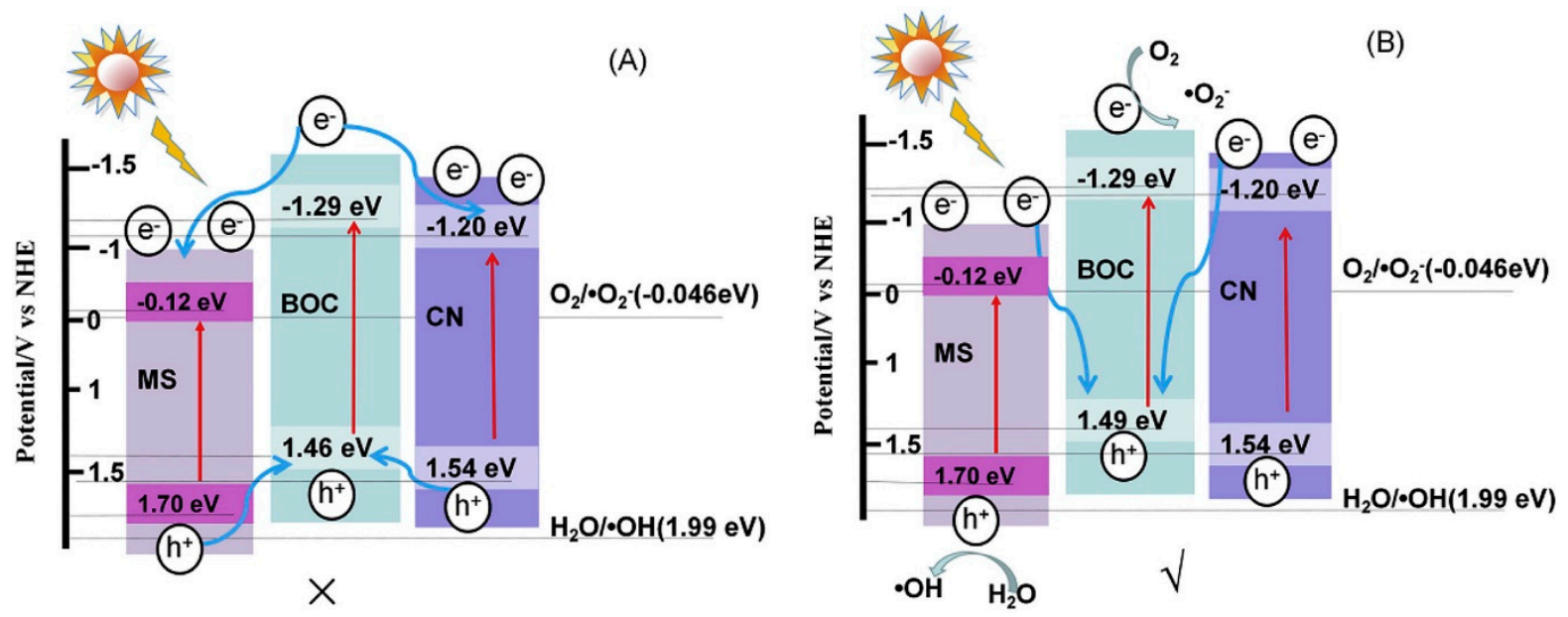

Figure 14. Schematic representation of the possible steps involved during the photocatalytic process and the charge transfer mechanism in the $\mathrm{g}-\mathrm{C}_{3} \mathrm{~N}_{4} / \mathrm{MoS}_{2} / \mathrm{Bi}_{24} \mathrm{O}_{31} \mathrm{Cl}_{10}$ composite: (A) traditional pathway and (B) dual Z-scheme pathway. Reprinted from Ref. [155] with permission from Elsevier.

Recent reports on nanostructured heterojunctions of g- $\mathrm{C}_{3} \mathrm{~N}_{4} / \mathrm{BiOI}$ show their poor dispersion in water, and they easily aggregate because of their higher surface energy. This leads to a remarkable reduction in their photocatalytic activity. In contrast, 1D nanofibres with a high surface area and high aspect ratios have potential in overcoming these problems. In particular, the 3D macroscopic structure of electrospun polyacrylonitrile nanofibres with excellent hydrophobicity can minimize agglomeration and improve the separation of the nanostructured heterojunctions of $\mathrm{g}-\mathrm{C}_{3} \mathrm{~N}_{4} / \mathrm{BiOI}$ in water for practical applications [150]. The fabrication of sandwich-like $\mathrm{BiOI} / \mathrm{AgI} / \mathrm{g}-\mathrm{C}_{3} \mathrm{~N}_{4}$ through the in situ crystallization approach showed good photocatalytic performance in degrading $\mathrm{MO}$ and the reduction of $\mathrm{Cr}(\mathrm{VI})$ ions under visible light irradiation. The $\mathrm{AgI}$ in the composite served as a charge transmission bridge between $\mathrm{BiOI}$ and g- $\mathrm{C}_{3} \mathrm{~N}_{4}$ that resulted in more efficient charge transfer and better separation of charge carriers [152]. Jiang et al. developed a novel ternary $\mathrm{BiOI} / \mathrm{g}-\mathrm{C}_{3} \mathrm{~N}_{4} / \mathrm{CeO}_{2}$ photocatalyst through calcination and hydrothermal treatment. This composite exhibited superior photocatalytic performance, which was far higher than that of either the single component or two component systems. For this photocatalytic $\mathrm{BiOI} / \mathrm{g}-\mathrm{C}_{3} \mathrm{~N}_{4} / \mathrm{CeO}_{2}$ heterojunction system, $91.6 \%$ of tetracycline was degraded in $120 \mathrm{~min}$, owing to the double charge transfer process between the g- $\mathrm{C}_{3} \mathrm{~N}_{4}$ and the other catalysts in the ternary heterojunction and the enhanced separation efficiency of photogenerated electron-hole pairs [153].

\subsection{Coupling BiOX and $\mathrm{BiOY}$ with $g-\mathrm{C}_{3} \mathrm{~N}_{4}$}

The approach of coupling two semiconductors to form a layered structure with an interfacial electric field is particularly promising since this enhances the possibility of satisfying the band alignment requirements for water splitting through the band structure, and subsequently boosts the separation between the photogenerated electron-hole pairs. In this context, heterolayers of $\mathrm{BiOX}_{1} / \mathrm{BiOX}_{2}$ (with $\mathrm{X}_{1}$ and $\mathrm{X}_{2}$ being different halides) are plausibly superior in comparison to homogeneous BiOX bilayers owing to the possibility of heterojunction induced separation of photogenerated electron-hole pairs [241]. For instance, a ternary heterojunction between $\mathrm{BiOI}, \mathrm{BiOCl}$ and $\mathrm{g}-\mathrm{C}_{3} \mathrm{~N}_{4}$ with different weight ratios was fabricated through the precipitation technique, among which $\mathrm{BiOI}(50)-\mathrm{BiOCl}(30) / \mathrm{g}$ - 
$\mathrm{C}_{3} \mathrm{~N}_{4}(20)$ exhibited enhanced photodegradation of acid orange 7 (10 ppm, 97\% in $140 \mathrm{~min}$ ) under visible light irradiation in comparison to pristine and other binary/ternary heterojunction counterparts. Under visible light exposure, the photogenerated $\mathrm{e}^{-}$in the $\mathrm{CB}$ of g- $\mathrm{C}_{3} \mathrm{~N}_{4}$ with low reduction ability were transferred to the $\mathrm{CB}$ of $\mathrm{BiOCl}$ and $\mathrm{BiOI}$, while the $\mathrm{h}^{+}$with low oxidation ability were transferred from the VB of BiOI to the VB of g- $\mathrm{C}_{3} \mathrm{~N}_{4}$, resulting in efficient charge separation and enabling the $\mathrm{e}^{-}$and $\mathrm{h}^{+}$with higher reduction and oxidation capabilities to participate in the photodegradation of acid orange 7 [91]. Similarly, a ternary composite of $\mathrm{g}-\mathrm{C}_{3} \mathrm{~N}_{4} / \mathrm{BiOI} / \mathrm{BiOBr}$ synthesized through the hydrothermal approach exhibited enhanced performance in the photodegradation of methylene blue (20 ppm, 80\% in $150 \mathrm{~min}$ ) under visible light irradiation. Matching band positions of g- $\mathrm{C}_{3} \mathrm{~N}_{4}$, $\mathrm{BiOI}$ and $\mathrm{BiOBr}$ allowed the transfer of charge carriers through a direct Z-scheme that favoured the efficient separation and transfer of the photogenerated charge carriers for the effective generation of reactive oxygen species [167]. The deposition-precipitation process was reported for synthesizing $2 \mathrm{D} \mathrm{g}-\mathrm{C}_{3} \mathrm{~N}_{4} @ \mathrm{BiOCl} / \mathrm{Bi}_{12} \mathrm{O}_{17} \mathrm{Cl}_{2}$ composites that were employed in the removal of nitric oxide with fixed concentration mixed with air stream under ambient temperature in a continuous flow reactor. The nitric oxide removal efficiency using the g- $\mathrm{C}_{3} \mathrm{~N}_{4} @ \mathrm{BiOCl} / \mathrm{Bi}_{12} \mathrm{O}_{17} \mathrm{Cl}_{2}$ heterojunction photocatalyst $(46.8 \%$ in $30 \mathrm{~min}$ ) was found to be greater than pristine $\mathrm{BiOCl} / \mathrm{Bi}_{12} \mathrm{O}_{17} \mathrm{Cl}_{2}(36.2 \%)$ and $\mathrm{g}-\mathrm{C}_{3} \mathrm{~N}_{4}(14.6 \%)$ under visible light irradiation, and was attributed to the intimate contact interface, suitable band structure, larger pore volume and improved visible light absorption [165]. Chou et al. reported the fabrication of various types of $\mathrm{BiO}_{\mathrm{x}} \mathrm{I}_{\mathrm{y}} / \mathrm{g}-\mathrm{C}_{3} \mathrm{~N}_{4}$ heterojunction composites through the hydrothermal method towards the photodegradation of crystal violet. Interestingly, $\mathrm{Bi}_{7} \mathrm{O}_{9} \mathrm{I}_{3} / \mathrm{Bi}_{5} \mathrm{O}_{7} \mathrm{I} / \mathrm{g}-\mathrm{C}_{3} \mathrm{~N}_{4}$ was found to exhibit superior performance in comparison to $\mathrm{BiOI} / \mathrm{g}-\mathrm{C}_{3} \mathrm{~N}_{4}, \mathrm{Bi}_{7} \mathrm{O}_{9} \mathrm{I}_{3} / \mathrm{g}-\mathrm{C}_{3} \mathrm{~N}_{4}$ and $\mathrm{Bi}_{5} \mathrm{O}_{7} \mathrm{I} / \mathrm{g}-\mathrm{C}_{3} \mathrm{~N}_{4}$ under visible light irradiation, which was ascribed primarily to the formation of a synergistic ternary heterojunction that ensured the separation of photogenerated charge carriers [163].

\section{Conclusions and Future Perspectives}

Recent years have witnessed significant progress in visible light driven photocatalysis aided by the comprehensive understanding of the structure-to-property relationship of nanostructured materials. Progress on molecularly thin 2D nanosheets has been phenomenal since the discovery of graphene, and studies in the past decade explored their customizable ultrathin architecture, composition and functionality driven by the exceptional physical, chemical, optical and electronic properties arising due to the unique ability of the nanosheets to confine electrons. Advancement towards 2D/2D heterojunction photocatalysts originated as a solution for tackling the rapid recombination of the photogenerated charge carriers in single component systems. However, many interesting studies pertaining to various $2 \mathrm{D}$ nanostructured photocatalysts are being pursued, and herein we have presented a comprehensive overview on the recent advances in the design, preparation, and photocatalytic applications of $\mathrm{BiOX} / \mathrm{Bi}_{\mathrm{x}} \mathrm{O}_{\mathrm{y}} \mathrm{X}_{\mathrm{z}}-\mathrm{g}-\mathrm{C}_{3} \mathrm{~N}_{4}$ heterojunction photocatalysts. The band structure of the individual components, the resulting properties and plausible outcomes during heterojunction formation were summarized. Then, various methods for fabricating $\mathrm{BiOX} / \mathrm{Bi}_{\mathrm{x}} \mathrm{O}_{\mathrm{y}} \mathrm{X}_{\mathrm{z}}-\mathrm{g}-\mathrm{C}_{3} \mathrm{~N}_{4}$ heterojunction photocatalysts were thoroughly discussed, emphasizing the dimensional anisotropy and morphological evolution that led to enhanced performance. Applications of the $\mathrm{BiOX} / \mathrm{Bi}_{\mathrm{x}} \mathrm{O}_{\mathrm{y}} \mathrm{X}_{\mathrm{z}}-\mathrm{g}-\mathrm{C}_{3} \mathrm{~N}_{4}$ photocatalysts in the degradation of various organic contaminants, $\mathrm{H}_{2}$ generation, $\mathrm{CO}_{2}$ reduction, $\mathrm{N}_{2}$ fixation and organic synthesis were summarized. Further, the improvement in the performance of $\mathrm{BiOX} / \mathrm{Bi}_{\mathrm{x}} \mathrm{O}_{\mathrm{y}} \mathrm{X}_{\mathrm{z}}-\mathrm{g}-\mathrm{C}_{3} \mathrm{~N}_{4}$ due to defects, facets and by the integration of metals, semiconductors and carbon materials is emphasized. The formation of the type-II heterojunction and Z-scheme bridge complimented with their structural stability is specified at relevant sections, and several salient studies are featured to stimulate the desire of the researchers to find a breakthrough.

Several studies were reported on the usage of $\mathrm{BiOX} / \mathrm{Bi}_{\mathrm{x}} \mathrm{O}_{\mathrm{y}} \mathrm{X}_{\mathrm{z}}-\mathrm{g}-\mathrm{C}_{3} \mathrm{~N}_{4}$ heterojunction photocatalysts for organic contaminant degradation, and some reports were available on the 
photocatalytic reduction of $\mathrm{CO}_{2}$ as summarized in Tables 1 and 2. Despite the encouraging results on photocatalytic $\mathrm{H}_{2}$ generation, $\mathrm{O}_{2}$ evolution and $\mathrm{N}_{2}$ fixation using $\mathrm{Bi}_{\mathrm{x}} \mathrm{O}_{\mathrm{y}} \mathrm{X}_{\mathrm{z}}$ and $\mathrm{g}-\mathrm{C}_{3} \mathrm{~N}_{4}$, it was surprising that no studies were reported to date on $\mathrm{BiOX} / \mathrm{Bi}_{\mathrm{x}} \mathrm{O}_{\mathrm{y}} \mathrm{X}_{\mathrm{z}}-\mathrm{g}-$ $\mathrm{C}_{3} \mathrm{~N}_{4}$ heterojunction photocatalysts. Therefore, plenty of opportunity exists for designing efficient heterojunction photocatalysts by strategically coupling engineered $\mathrm{Bi}_{\mathrm{x}} \mathrm{O}_{\mathrm{y}} \mathrm{X}_{\mathrm{z}}$ and g- $\mathrm{C}_{3} \mathrm{~N}_{4}$, as detailed below.

(1) Many recent studies reported the synthesis of defect-rich $g-\mathrm{C}_{3} \mathrm{~N}_{4}$ for realizing enhanced activity for $\mathrm{H}_{2}$ generation [242-245]. The introduction of defects in the form of nitrogen vacancies in $\mathrm{g}-\mathrm{C}_{3} \mathrm{~N}_{4}$ induced the formation of midgap states under the $\mathrm{CB}$ that resulted in the extension of the visible light absorption, and trapped the photogenerated $\mathrm{e}^{-}$to minimize recombination loss while facilitating its rapid transfer. Forming a heterojunction by combining defect-rich g- $\mathrm{C}_{3} \mathrm{~N}_{4}$ with defect-rich $\mathrm{BiOX} / \mathrm{Bi}_{\mathrm{x}} \mathrm{O}_{\mathrm{y}} \mathrm{X}_{\mathrm{z}}$ can enhance $\mathrm{H}_{2}$ generation.

(2) The inherent drawback of $\mathrm{g}-\mathrm{C}_{3} \mathrm{~N}_{4}$ has been its poor mass diffusion and charge separation efficiency for achieving enhanced photocatalytic $\mathrm{O}_{2}$ evolution efficiency. Modulating the band structures of $\mathrm{g}-\mathrm{C}_{3} \mathrm{~N}_{4}$ (by protonation or the addition of defects and dopants) was reported to enhance its efficiency [246], and therefore a heterojunction photocatalyst constructed between $\mathrm{Bi}_{\mathrm{x}} \mathrm{O}_{\mathrm{y}} \mathrm{X}_{\mathrm{z}}$ with exposed facets and band structure modulated g- $\mathrm{C}_{3} \mathrm{~N}_{4}$ could achieve enhanced quantum efficiencies.

(3) Despite the CB of g- $\mathrm{C}_{3} \mathrm{~N}_{4}$ being more negative than $\mathrm{N}_{2} / \mathrm{NH}_{3}$ reduction potential, its low conductivity and high recombination rate are some of the impediments that deter its potential for photocatalytic $\mathrm{N}_{2}$ fixation. The concurrent addition of dopants and defects (carbon/nitrogen vacancies) was reported to improve its photocatalytic $\mathrm{N}_{2}$ fixation efficiency $[247,248]$. Heterojunction photocatalysts constructed between doped $\mathrm{BiOX} / \mathrm{Bi}_{\mathrm{x}} \mathrm{O}_{\mathrm{y}} \mathrm{X}_{\mathrm{z}}$ with exposed facets and doped/defect-rich $\mathrm{g}-\mathrm{C}_{3} \mathrm{~N}_{4}$ are expected to exhibit enhanced photocatalytic $\mathrm{N}_{2}$ fixation efficiency.

(4) Another interesting opportunity is the construction of a heterojunction between an atomically thin layer of $\mathrm{Bi}_{\mathrm{x}} \mathrm{O}_{\mathrm{y}} \mathrm{X}_{\mathrm{z}}$ and $\mathrm{g}-\mathrm{C}_{3} \mathrm{~N}_{4}$, which can be very challenging. However, the unique physical and chemical properties in addition to the easy formation of surface defects could pave the way towards enhanced quantum efficiencies.

Funding: This research received no external funding.

Acknowledgments: This work was supported by the Department of Science and Technology, Government of India through the DST INSPIRE Faculty project (No. IFA15 MS-41) and was funded by the MEXT Promotion of Distinctive Joint Research Center Program Grant Number JPMXP 0618217662. SP acknowledges the European Regional Development Grant for providing Ser Cymru-II Rising Star Fellowship through the Welsh Government (80761-SU-102-West).

Conflicts of Interest: The authors declare no conflict of interest.

\section{References}

1. Taylor, D. The Pharmaceutical Industry and the Future of Drug Development. Issues Environ. Sci. Technol. 2016, $2016,1-33$.

2. Zhang, G.; Wang, J.; Zhang, H.; Zhang, T.; Jiang, S.; Li, B.; Zhang, H.; Cao, J. Facile Synthesize Hierarchical Tubular Micro-Nano Structured $\mathrm{AgCl} / \mathrm{Ag} / \mathrm{TiO} 2$ Hybrid with Favorable Visible Light Photocatalytic Performance. J. Alloys Compd. 2021, 855, 157512. [CrossRef]

3. Zhang, Q.-Q.; Ying, G.-G.; Pan, C.-G.; Liu, Y.-S.; Zhao, J.-L. Comprehensive Evaluation of Antibiotics Emission and Fate in the River Basins of China: Source Analysis, Multimedia Modeling, and Linkage to Bacterial Resistance. Environ. Sci. Technol. 2015, 49, 6772-6782. [CrossRef] [PubMed]

4. Bound, J.P.; Voulvoulis, N. Pharmaceuticals in the Aquatic Environment-A Comparison of Risk Assessment Strategies. Chemosphere 2004, 56, 1143-1155. [CrossRef] [PubMed]

5. Saxena, R.; Saxena, M.; Lochab, A. Recent Progress in Nanomaterials for Adsorptive Removal of Organic Contaminants from Wastewater. ChemistrySelect 2020, 5, 335-353. [CrossRef]

6. Kanaujiya, D.K.; Paul, T.; Sinharoy, A.; Pakshirajan, K. Biological Treatment Processes for the Removal of Organic Micropollutants from Wastewater: A Review. Curr. Pollut. Rep. 2019, 5, 112-128. [CrossRef]

7. Deng, Y.; Zhao, R. Advanced Oxidation Processes (AOPs) in Wastewater Treatment. Curr. Pollut. Rep. 2015, 1, 167-176. [CrossRef]

8. Fujishima, A.; Honda, K. Electrochemical Photolysis of Water at a Semiconductor Electrode. Nature 1972, 238, 37-38. [CrossRef] 
9. Dong, S.; Feng, J.; Fan, M.; Pi, Y.; Hu, L.; Han, X.; Liu, M.; Sun, J.; Sun, J. Recent Developments in Heterogeneous Photocatalytic Water Treatment Using Visible Light-Responsive Photocatalysts: A Review. RSC Adv. 2015, 5, 14610-14630. [CrossRef]

10. Chatterjee, D.; Dasgupta, S. Visible Light Induced Photocatalytic Degradation of Organic Pollutants. J. Photochem. Photobiol. C Photochem. Rev. 2005, 6, 186-205. [CrossRef]

11. Bora, L.V.; Mewada, R.K. Visible/Solar Light Active Photocatalysts for Organic Effluent Treatment: Fundamentals, Mechanisms and Parametric Review. Renew. Sustain. Energy Rev. 2017, 76, 1393-1421. [CrossRef]

12. Ong, W.-J.; Shak, K.P.Y. 2D/2D Heterostructured Photocatalysts: An Emerging Platform for Artificial Photosynthesis. Sol. Rrl 2020, 4, 2000132. [CrossRef]

13. Li, X.; Yu, J.; Wageh, S.; Al-Ghamdi, A.A.; Xie, J. Graphene in Photocatalysis: A Review. Small 2016, 12, 6640-6696. [CrossRef]

14. Di, J.; Xia, J.; Li, H.; Guo, S.; Dai, S. Bismuth Oxyhalide Layered Materials for Energy and Environmental Applications. Nano Energy 2017, 41, 172-192. [CrossRef]

15. Ye, L.; Su, Y.; Jin, X.; Xie, H.; Zhang, C. Recent Advances in BiOX (X = Cl, Br and I) Photocatalysts: Synthesis, Modification, Facet Effects and Mechanisms. Environ. Sci. Nano 2014, 1, 90-112. [CrossRef]

16. Li, J.; Yu, Y.; Zhang, L. Bismuth Oxyhalide Nanomaterials: Layered Structures Meet Photocatalysis. Nanoscale 2014, 6, 8473-8488. [CrossRef]

17. Gondal, M.A.; Xiaofeng, C.; Dastageer, M.A. Novel Bismuth-Oxyhalide-Based Materials and Their Applications, Advanced Structured Materials; Springer: New Delhi, India, 2017; Volume 76, ISBN 978-81-322-3737-2.

18. Guo, M.; He, H.; Cao, J.; Lin, H.; Chen, S. Novel I-Doped Bi12O17Cl2 Photocatalysts with Enhanced Photocatalytic Activity for Contaminants Removal. Mater. Res. Bull. 2019, 112, 205-212. [CrossRef]

19. Ong, W.J.; Tan, L.L.; Ng, Y.H.; Yong, S.T.; Chai, S.P. Graphitic Carbon Nitride (g-C3N4)-Based Photocatalysts for Artificial Photosynthesis and Environmental Remediation: Are We a Step Closer to Achieving Sustainability? Chem. Rev. 2016, 116, 7159-7329. [CrossRef]

20. Mishra, A.; Mehta, A.; Basu, S.; Shetti, N.P.; Reddy, K.R.; Aminabhavi, T.M. Graphitic Carbon Nitride (g-C3N4)-Based Metal-Free Photocatalysts for Water Splitting: A Review. Carbon 2019, 149, 693-721. [CrossRef]

21. Yi, J.; El-Alami, W.; Song, Y.; Li, H.; Ajayan, P.M.; Xu, H. Emerging Surface Strategies on Graphitic Carbon Nitride for Solar Driven Water Splitting. Chem. Eng. J. 2020, 382, 122812. [CrossRef]

22. Liu, J.; Wang, H.; Antonietti, M. Graphitic Carbon Nitride "Reloaded": Emerging Applications beyond (Photo)Catalysis. Chem. Soc. Rev. 2016, 45, 2308-2326. [CrossRef] [PubMed]

23. Ong, W.J. 2D/2D Graphitic Carbon Nitride (g-C3 N4) Heterojunction Nanocomposites for Photocatalysis: Why Does Face-to-Face Interface Matter? Front. Mater. 2017, 4, 11. [CrossRef]

24. Yao, W.; Zhang, J.; Wang, Y.; Ren, F. Hybrid Density Functional Study on the Mechanism for the Enhanced Photocatalytic Properties of the Ultrathin Hybrid Layered Nanocomposite G-C3N4/BiOCl. Appl. Surf. Sci. 2018, 435, 1351-1360. [CrossRef]

25. Zheng, C.Z.; Zhang, C.Y.; Zhang, G.H.; Zhao, D.J.; Wang, Y.Z. Enhanced Photocatalytic Performance of G-C3N4 with BiOCl Quantum Dots Modification. Mater. Res. Bull. 2014, 55, 212-215. [CrossRef]

26. Shi, S.; Gondal, M.A.; Al-Saadi, A.A.; Fajgar, R.; Kupcik, J.; Chang, X.; Shen, K.; Xu, Q.; Seddigi, Z.S. Facile Preparation of G-C3N4 Modified BiOCl Hybrid Photocatalyst and Vital Role of Frontier Orbital Energy Levels of Model Compounds in Photoactivity Enhancement. J. Colloid Interface Sci. 2014, 416, 212-219. [CrossRef] [PubMed]

27. Zhang, H.; Zhao, L.; Wang, L.; Hao, J.; Meng, X. Fabrication of Oxygen-Vacancy-Rich Black-BiOBr/BiOBr Heterojunction with Enhanced Photocatalytic Activity. J. Mater. Sci. 2020, 55, 10785-10795. [CrossRef]

28. Yang, W.; Shan, X.; Chen, Y.; Gao, Y. Enhanced Photocatalytic Performance of C3N4 via Doping with $\pi$-Deficient Conjugated Pyridine Ring and BiOCl Composite Heterogeneous Materials. Diam. Relat. Mater. 2020, 108, 107926. [CrossRef]

29. Song, L.; Zheng, Y.; Chen, C. Sonication-Assisted Deposition-Precipitation Synthesis of Graphitic C3N4/BiOCl Heterostructured Photocatalysts with Enhanced Rhodamine B Photodegradation Activity. J. Mater. Sci. Mater. Electron. 2017, 28, 15861-15869. [CrossRef]

30. Liu, R.; Chen, Z.; Yao, Y.; Li, Y.; Cheema, W.A.; Wang, D.; Zhu, S. Recent Advancements in G-C3N4-Based Photocatalysts for Photocatalytic CO2reduction: A Mini Review. RSC Adv. 2020, 10, 29408-29418. [CrossRef]

31. Zhang, X.; Yuan, X.; Jiang, L.; Zhang, J.; Yu, H.; Wang, H.; Zeng, G. Powerful Combination of 2D G-C3N4 and 2D Nanomaterials for Photocatalysis: Recent Advances. Chem. Eng. J. 2020, 390, 124475. [CrossRef]

32. Lam, S.S.; Nguyen, V.H.; Nguyen Dinh, M.T.; Khieu, D.Q.; La, D.D.; Nguyen, H.T.; Vo, D.V.N.; Xia, C.; Varma, R.S.; Shokouhimehr M.; et al. Mainstream Avenues for Boosting Graphitic Carbon Nitride Efficiency: Towards Enhanced Solar Light-Driven Photocatalytic Hydrogen Production and Environmental Remediation. J. Mater. Chem. A 2020, 8, 10571-10603. [CrossRef]

33. Chen, Z.; Zhang, S.; Liu, Y.; Alharbi, N.S.; Rabah, S.O.; Wang, S.; Wang, X. Synthesis and Fabrication of G-C3N4-Based Materials and Their Application in Elimination of Pollutants. Sci. Total Environ. 2020, 731, 139054. [CrossRef]

34. Fronczak, M. Adsorption Performance of Graphitic Carbon Nitride-Based Materials: Current State of the Art. J. Environ. Chem. Eng. 2020, 8, 104411. [CrossRef]

35. Stroyuk, O.; Raievska, O.; Zahn, D.R.T. Graphitic Carbon Nitride Nanotubes: A New Material for Emerging Applications. RSC Adv. 2020, 10, 34059-34087. [CrossRef]

36. Kong, L.; Song, P.; Ma, F.; Sun, M. Graphitic Carbon Nitride-Based 2D Catalysts for Green Energy: Physical Mechanism and Applications. Mater. Today Energy 2020, 17, 100488. [CrossRef] 
37. Starukh, H.; Praus, P. Doping of Graphitic Carbon Nitride with Non-Metal Elements and Its Applications in Photocatalysis. Catalysts 2020, 10, 1119. [CrossRef]

38. Huang, X.; Gu, W.; Ma, Y.; Liu, D.; Ding, N.; Zhou, L.; Lei, J.; Wang, L.; Zhang, J. Recent Advances of Doped Graphite Carbon Nitride for Photocatalytic Reduction of CO2: A Review. Res. Chem. Intermed. 2020, 46, 5133-5164. [CrossRef]

39. Ismael, M. A Review on Graphitic Carbon Nitride (g-C3N4) Based Nanocomposites: Synthesis, Categories, and Their Application in Photocatalysis. J. Alloys Compd. 2020, 846, 156446. [CrossRef]

40. Zhang, W.; Mohamed, A.R.; Ong, W. Z-Scheme Photocatalytic Systems for Carbon Dioxide Reduction: Where Are We Now? Angew. Chem. Int. Ed. 2020, anie.201914925. [CrossRef]

41. Li, Y.; Zhou, M.; Cheng, B.; Shao, Y. Recent Advances in G-C3N4-Based Heterojunction Photocatalysts. J. Mater. Sci. Technol. 2020, 56,1-17. [CrossRef]

42. Li, Y.; Li, X.; Zhang, H.; Fan, J.; Xiang, Q. Design and Application of Active Sites in G-C3N4-Based Photocatalysts. J. Mater. Sci. Technol. 2020, 56, 69-88. [CrossRef]

43. Jourshabani, M.; Lee, B.K.; Shariatinia, Z. From Traditional Strategies to Z-Scheme Configuration in Graphitic Carbon Nitride Photocatalysts: Recent Progress and Future Challenges. Appl. Catal. B Environ. 2020, 276, 119157. [CrossRef]

44. Singh, S.; Sharma, R.; Khanuja, M. A Review and Recent Developments on Strategies to Improve the Photocatalytic Elimination of Organic Dye Pollutants by BiOX (X = Cl, Br, I, F) Nanostructures. Korean J. Chem. Eng. 2018, 35, 1955-1968. [CrossRef]

45. Bismuth Oxyhalide Compounds as Photocatalysts-«Progress in Chemistry $\gg$. September 2009. Available online: https: / / en.cnki.com.cn/Article_en/CJFDTotal-HXJZ200909004.htm (accessed on 21 November 2020).

46. Liu, J.Q.; Wu, Y.C. Recent Advances in the High Performance BiOX (X = Cl, Br, I) Based Photo-Catalysts. Wuji Cailiao Xuebao/J. Inorg. Mater. 2015, 30, 1009-1017.

47. Cheng, H.; Huang, B.; Dai, Y. Engineering BiOX (X=Cl, Br, I) Nanostructures for Highly Efficient Photocatalytic Applications. Nanoscale 2014, 6, 2009-2026. [CrossRef]

48. Zhao, Z.; Sun, Y.; Dong, F. Graphitic Carbon Nitride Based Nanocomposites: A Review. Nanoscale 2015, 7, 15-37. [CrossRef]

49. Meng, X.; Zhang, Z. Bismuth-Based Photocatalytic Semiconductors: Introduction, Challenges and Possible Approaches. J. Mol. Catal. A Chem. 2016, 423, 533-549. [CrossRef]

50. Chen, Y.; Jia, G.; Hu, Y.; Fan, G.; Tsang, Y.H.; Li, Z.; Zou, Z. Two-Dimensional Nanomaterials for Photocatalytic CO2 Reduction to Solar Fuels. Sustain. Energy Fuels 2017, 1, 1875-1898. [CrossRef]

51. Yang, Y.; Zhang, C.; Lai, C.; Zeng, G.; Huang, D.; Cheng, M.; Wang, J.; Chen, F.; Zhou, C.; Xiong, W. BiOX (X = Cl, Br, I) Photocatalytic Nanomaterials: Applications for Fuels and Environmental Management. Adv. Colloid Interface Sci. 2018, 254, 76-93. [CrossRef]

52. Arthur, R.; Ahern, J.; Patterson, H. Application of BiOX Photocatalysts in Remediation of Persistent Organic Pollutants. Catalysts 2018, 8, 604. [CrossRef]

53. Garg, S.; Yadav, M.; Chandra, A.; Hernadi, K. A Review on BiOX (X=Cl, Br and I) Nano-/Microstructures for Their Photocatalytic Applications. J. Nanosci. Nanotechnol. 2018, 19, 280-294. [CrossRef] [PubMed]

54. Ye, L.; Deng, Y.; Wang, L.; Xie, H.; Su, F. Bismuth-Based Photocatalysts for Solar Photocatalytic Carbon Dioxide Conversion. ChemSusChem 2019, 12, 3671-3701. [CrossRef] [PubMed]

55. Wang, Z.; Chen, M.; Huang, D.; Zeng, G.; Xu, P.; Zhou, C.; Lai, C.; Wang, H.; Cheng, M.; Wang, W. Multiply Structural Optimized Strategies for Bismuth Oxyhalide Photocatalysis and Their Environmental Application. Chem. Eng. J. 2019, 374, 1025-1045. [CrossRef]

56. Sharma, K.; Dutta, V.; Sharma, S.; Raizada, P.; Hosseini-Bandegharaei, A.; Thakur, P.; Singh, P. Recent Advances in Enhanced Photocatalytic Activity of Bismuth Oxyhalides for Efficient Photocatalysis of Organic Pollutants in Water: A Review. J. Ind. Eng. Chem. 2019, 78, 1-20. [CrossRef]

57. Zhao, Y.; Zhang, S.; Shi, R.; Waterhouse, G.I.N.; Tang, J.; Zhang, T. Two-Dimensional Photocatalyst Design: A Critical Review of Recent Experimental and Computational Advances. Mater. Today 2020, 34, 78-91. [CrossRef]

58. Xiong, J.; Song, P.; Di, J.; Li, H. Bismuth-Rich Bismuth Oxyhalides: A New Opportunity to Trigger High-Efficiency Photocatalysis. J. Mater. Chem. A 2020, 8, 21434-21454. [CrossRef]

59. Ren, K.; Liu, J.; Liang, J.; Zhang, K.; Zheng, X.; Luo, H.; Huang, Y.; Liu, P.; Yu, X. Synthesis of the Bismuth Oxyhalide Solid Solutions with Tunable Band Gap and Photocatalytic Activities. Dalton Trans. 2013, 42, 9706-9712. [CrossRef]

60. Shi, L.; Si, W.; Wang, F.; Qi, W. Construction of 2D/2D Layered g-C3N4/Bi12O17Cl2 Hybrid Material with Matched Energy Band Structure and Its Improved Photocatalytic Performance. RSC Adv. 2018, 8, 24500-24508. [CrossRef]

61. Chen, X.; Zhang, J.; Liu, L.; Hu, B.; Zhao, Y.; Zhao, S.; Zhao, W.; Li, S.; Hai, X. Tailored Fabrication of Interface-Rich Hierarchical Bi24O31Br10 with Enhanced Photocatalytic Performance. Appl. Surf. Sci. 2019, 491, 1-8. [CrossRef]

62. Yang, L.; Liang, L.; Wang, L.; Zhu, J.; Gao, S.; Xia, X. Accelerated Photocatalytic Oxidation of Carbamazepine by a Novel 3D Hierarchical Protonated G-C3N4/BiOBr Heterojunction: Performance and Mechanism. Appl. Surf. Sci. 2019, 473, 527-539. [CrossRef]

63. Hu, L.; He, H.; Xia, D.; Huang, Y.; Xu, J.; Li, H.; He, C.; Yang, W.; Shu, D.; Wong, P.K. Highly Efficient Performance and Conversion Pathway of Photocatalytic CH3SH Oxidation on Self-Stabilized Indirect Z-Scheme g-C3N4/I3-BiOI. Acs Appl. Mater. Interfaces 2018, 10, 18693-18708. [CrossRef] 
64. Yin, R.; Li, Y.; Zhong, K.; Yao, H.; Zhang, Y.; Lai, K. Multifunctional Property Exploration: Bi4O5I2 with High Visible Light Photocatalytic Performance and a Large Nonlinear Optical Effect. Rsc Adv. 2019, 9, 4539-4544. [CrossRef]

65. Di, J.; Zhu, C.; Ji, M.; Duan, M.; Long, R.; Yan, C.; Gu, K.; Xiong, J.; She, Y.; Xia, J.; et al. Defect-Rich Bi12O17Cl2 Nanotubes Self-Accelerating Charge Separation for Boosting Photocatalytic CO2 Reduction. Angew. Chem. Int. Ed. 2018, 57, 14847-14851. [CrossRef]

66. Shang, J.; Hao, W.; Lv, X.; Wang, T.; Wang, X.; Du, Y.; Dou, S.; Xie, T.; Wang, D.; Wang, J. Bismuth Oxybromide with Reasonable Photocatalytic Reduction Activity under Visible Light. Acs Catal. 2014, 4, 954-961. [CrossRef]

67. Bai, Y.; Chen, T.; Wang, P.; Wang, L.; Ye, L. Bismuth-Rich Bi4O5X2 (X = Br, and I) Nanosheets with Dominant $\{101\}$ Facets Exposure for Photocatalytic H2 Evolution. Chem. Eng. J. 2016, 304, 454-460. [CrossRef]

68. Mi, Y.; Li, H.; Zhang, Y.; Hou, W. Synthesis of Belt-like Bismuth-Rich Bismuth Oxybromide Hierarchical Nanostructures with High Photocatalytic Activities. J. Colloid Interface Sci. 2019, 534, 301-311. [CrossRef]

69. Wang, X.; Maeda, K.; Thomas, A.; Takanabe, K.; Xin, G.; Carlsson, J.M.; Domen, K.; Antonietti, M. A Metal-Free Polymeric Photocatalyst for Hydrogen Production from Water under Visible Light. Nat. Mater. 2009, 8, 76-80. [CrossRef] [PubMed]

70. Teixeira, I.F.; Barbosa, E.C.M.; Tsang, S.C.E.; Camargo, P.H.C. Carbon Nitrides and Metal Nanoparticles: From Controlled Synthesis to Design Principles for Improved Photocatalysis. Chem. Soc. Rev. 2018, 47, 7783-7817. [CrossRef]

71. Wen, J.; Xie, J.; Chen, X.; Li, X. A Review on G-C3 N4 -Based Photocatalysts. Appl. Surf. Sci. 2017, 391, 72-123. [CrossRef]

72. Yan, S.C.; Lv, S.B.; Li, Z.S.; Zou, Z.G. Organic-Inorganic Composite Photocatalyst of g-C3N4 and TaON with Improved Visible Light Photocatalytic Activities. Dalton Trans. 2010, 39, 1488-1491. [CrossRef] [PubMed]

73. Sudhaik, A.; Raizada, P.; Shandilya, P.; Jeong, D.-Y.; Lim, J.-H.; Singh, P. Review on Fabrication of Graphitic Carbon Nitride Based Efficient Nanocomposites for Photodegradation of Aqueous Phase Organic Pollutants. J. Ind. Eng. Chem. 2018, 67, 28-51. [CrossRef]

74. Dong, G.; Zhang, Y.; Pan, Q.; Qiu, J. A Fantastic Graphitic Carbon Nitride (g-C3N4) Material: Electronic Structure, Photocatalytic and Photoelectronic Properties. J. Photochem. Photobiol. C Photochem. Rev. 2014, 20, 33-50. [CrossRef]

75. You, Z.; Wu, C.; Shen, Q.; Yu, Y.; Chen, H.; Su, Y.; Wang, H.; Wu, C.; Zhang, F.; Yang, H. A Novel Efficient G-C3N4@BiOI p-n Heterojunction Photocatalyst Constructed through the Assembly of g-C3N4 Nanoparticles. Dalton Trans. 2018, 47, 7353-7361. [CrossRef]

76. Rabenau, A. The Role of Hydrothermal Synthesis in Preparative Chemistry. Angew. Chem. Int. Ed. Engl. 1985, 24, 1026-1040. [CrossRef]

77. Yang, Y.; Matsubara, S.; Xiong, L.; Hayakawa, T.; Nogami, M. Solvothermal Synthesis of Multiple Shapes of Silver Nanoparticles and Their SERS Properties. J. Phys. Chem. C 2007, 111, 9095-9104. [CrossRef]

78. Xiao, X.; Liu, C.; Hu, R.; Zuo, X.; Nan, J.; Li, L.; Wang, L. Oxygen-Rich Bismuth Oxyhalides: Generalized One-Pot Synthesis, Band Structures and Visible-Light Photocatalytic Properties. J. Mater. Chem. 2012, 22, 22840-22843. [CrossRef]

79. Huo, Y.; Zhang, J.; Miao, M.; Jin, Y. Solvothermal Synthesis of Flower-like BiOBr Microspheres with Highly Visible-Light Photocatalytic Performances. Appl. Catal. B Environ. 2012, 111-112, 334-341. [CrossRef]

80. Shi, X.; Chen, X.; Chen, X.; Zhou, S.; Lou, S.; Wang, Y.; Yuan, L. PVP Assisted Hydrothermal Synthesis of BiOBr Hierarchical Nanostructures and High Photocatalytic Capacity. Chem. Eng. J. 2013, 222, 120-127. [CrossRef]

81. Li, H.; Ma, A.; Zhang, D.; Gao, Y.; Dong, Y. Rational Design Direct Z-Scheme BiOBr/g-C3N4 Heterojunction with Enhanced Visible Photocatalytic Activity for Organic Pollutants Elimination. Rsc Adv. 2020, 10, 4681-4689. [CrossRef]

82. Li, X.; Yu, J.; Jaroniec, M. Hierarchical Photocatalysts. Chem. Soc. Rev. 2016, 45, 2603-2636. [CrossRef]

83. Wang, S.; Hai, X.; Ding, X.; Chang, K.; Xiang, Y.; Meng, X.; Yang, Z.; Chen, H.; Ye, J. Light-Switchable Oxygen Vacancies in Ultrafine Bi5O7Br Nanotubes for Boosting Solar-Driven Nitrogen Fixation in Pure Water. Adv. Mater. 2017, 29. [CrossRef]

84. Liu, H.; Zhou, H.; Liu, X.; Li, H.; Ren, C.; Li, X.; Li, W.; Lian, Z.; Zhang, M. Engineering Design of Hierarchical G-C3N4@Bi/BiOBr Ternary Heterojunction with Z-Scheme System for Efficient Visible-Light Photocatalytic Performance. J. Alloys Compd. 2019, 798, 741-749. [CrossRef]

85. Ji, M.; Di, J.; Ge, Y.; Xia, J.; Li, H. 2D-2D Stacking of Graphene-like g-C3N4/Ultrathin Bi4O5Br2 with Matched Energy Band Structure towards Antibiotic Removal. Appl. Surf. Sci. 2017, 413, 372-380. [CrossRef]

86. Wang, X.J.; Wang, Q.; Li, F.T.; Yang, W.Y.; Zhao, Y.; Hao, Y.J.; Liu, S.J. Novel BiOCl-C3N4 Heterojunction Photocatalysts: In Situ Preparation via an Ionic-Liquid-Assisted Solvent-Thermal Route and Their Visible-Light Photocatalytic Activities. Chem. Eng. J. 2013, 234, 361-371. [CrossRef]

87. Xia, J.; Ji, M.; Di, J.; Wang, B.; Yin, S.; Zhang, Q.; He, M.; Li, H. Construction of Ultrathin C3N4/Bi4O5I2 Layered Nanojunctions via Ionic Liquid with Enhanced Photocatalytic Performance and Mechanism Insight. Appl. Catal. B Environ. 2016, 191, 235-245. [CrossRef]

88. Di, J.; Xia, J.; Yin, S.; Xu, H.; He, M.; Li, H.; Xu, L.; Jiang, Y. A G-C3N4/BiOBr Visible-Light-Driven Composite: Synthesis via a Reactable Ionic Liquid and Improved Photocatalytic Activity. RSC Adv. 2013, 3, 19624-19631. [CrossRef]

89. Yin, S.; Di, J.; Li, M.; Sun, Y.; Xia, J.; Xu, H.; Fan, W.; Li, H. Ionic Liquid-Assisted Synthesis and Improved Photocatalytic Activity of p-n Junction g-C3N4/BiOCl. J. Mater. Sci. 2016, 51, 4769-4777. [CrossRef]

90. Ren, X.; Gao, M.; Zhang, Y.; Zhang, Z.; Cao, X.; Wang, B.; Wang, X. Photocatalytic Reduction of CO2 on BiOX: Effect of Halogen Element Type and Surface Oxygen Vacancy Mediated Mechanism. Appl. Catal. B Environ. 2020, 274, 119063. [CrossRef] 
91. Aghdam, S.M.; Haghighi, M.; Allahyari, S.; Yosefi, L. Precipitation Dispersion of Various Ratios of BiOI/BiOCl Nanocomposite over g-C3N4 for Promoted Visible Light Nanophotocatalyst Used in Removal of Acid Orange 7 from Water. J. Photochem. Photobiol. A Chem. 2017, 338, 201-212. [CrossRef]

92. Mousavi, M.; Habibi-Yangjeh, A. Magnetically Separable Ternary G-C3N4/Fe3O4/BiOI Nanocomposites: Novel Visible-LightDriven Photocatalysts Based on Graphitic Carbon Nitride. J. Colloid Interface Sci. 2016, 465, 83-92. [CrossRef]

93. Asadzadeh-Khaneghah, S.; Habibi-Yangjeh, A.; Yubuta, K. Novel G-C3N4 Nanosheets/CDs/BiOCl Photocatalysts with Exceptional Activity under Visible Light. J. Am. Ceram. Soc. 2019, 102, 1435-1453. [CrossRef]

94. Asadzadeh-Khaneghah, S.; Habibi-Yangjeh, A.; Nakata, K. Graphitic Carbon Nitride Nanosheets Anchored with BiOBr and Carbon Dots: Exceptional Visible-Light-Driven Photocatalytic Performances for Oxidation and Reduction Reactions. J. Colloid Interface Sci. 2018, 530, 642-657. [CrossRef]

95. Di, J.; Xia, J.; Chisholm, M.F.; Zhong, J.; Chen, C.; Cao, X.; Dong, F.; Chi, Z.; Chen, H.; Weng, Y.; et al. Defect-Tailoring Mediated

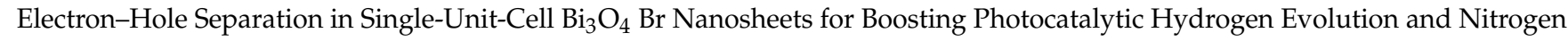
Fixation. Adv. Mater. 2019, 31, 1807576. [CrossRef]

96. Che, H.; Che, G.; Dong, H.; Hu, W.; Hu, H.; Liu, C.; Li, C. Fabrication of Z-Scheme Bi3O4Cl/g-C3N4 2D/2D Heterojunctions with Enhanced Interfacial Charge Separation and Photocatalytic Degradation Various Organic Pollutants Activity. Appl. Surf. Sci. 2018, 455, 705-716. [CrossRef]

97. Bang, J.H.; Suslick, K.S. Applications of Ultrasound to the Synthesis of Nanostructured Materials. Adv. Mater. 2010, 22, 1039-1059. [CrossRef]

98. Pereira, C.; Pereira, A.M.; Fernandes, C.; Rocha, M.; Mendes, R.; Fernández-García, M.P.; Guedes, A.; Tavares, P.B.; Grenéche, J.M.; Araújo, J.P.; et al. Superparamagnetic MFe $2 \mathrm{O} 4(\mathrm{M}=\mathrm{Fe}, \mathrm{Co}, \mathrm{Mn})$ Nanoparticles: Tuning the Particle Size and Magnetic Properties through a Novel One-Step Coprecipitation Route. Chem. Mater. 2012, 24, 1496-1504. [CrossRef]

99. Liu, X.; Ni, Z.; He, Y.; Su, N.; Guo, R.; Wang, Q.; Yi, T. Ultrasound-Assisted Two-Step Water-Bath Synthesis of g-C3N4/BiOBr Composites: Visible Light-Driven Photocatalysis, Sterilization, and Reaction Mechanism. New J. Chem. 2019, 43, 8711-8721. [CrossRef]

100. Pelaez, M.; Nolan, N.T.; Pillai, S.C.; Seery, M.K.; Falaras, P.; Kontos, A.G.; Dunlop, P.S.M.; Hamilton, J.W.J.; Byrne, J.A.; O’Shea, K.; et al. A Review on the Visible Light Active Titanium Dioxide Photocatalysts for Environmental Applications. Appl. Catal. B Environ. 2012, 125, 331-349. [CrossRef]

101. Banerjee, S.; Pillai, S.C.; Falaras, P.; O'shea, K.E.; Byrne, J.A.; Dionysiou, D.D. New Insights into the Mechanism of Visible Light Photocatalysis. J. Phys. Chem. Lett. 2014, 5, 2543-2554. [CrossRef] [PubMed]

102. Di, J.; Xia, J.; Ji, M.; Yin, S.; Li, H.; Xu, H.; Zhang, Q.; Li, H. Controllable Synthesis of Bi4O5Br2 Ultrathin Nanosheets for Photocatalytic Removal of Ciprofloxacin and Mechanism Insight. J. Mater. Chem. A 2015, 3, 15108-15118. [CrossRef]

103. Wang, C.Y.; Zhang, X.; Qiu, H.B.; Huang, G.X.; Yu, H.Q. Bi24O31Br10 Nanosheets with Controllable Thickness for Visible-LightDriven Catalytic Degradation of Tetracycline Hydrochloride. Appl. Catal. B Environ. 2017, 205, 615-623. [CrossRef]

104. Ye, L.; Liu, J.; Jiang, Z.; Peng, T.; Zan, L. Facets Coupling of BiOBr-g-C3N4 Composite Photocatalyst for Enhanced Visible-LightDriven Photocatalytic Activity. Appl. Catal. B Environ. 2013, 142-143, 1-7. [CrossRef]

105. Di, J.; Xia, J.; Yin, S.; Xu, H.; Xu, L.; Xu, Y.; He, M.; Li, H. Preparation of Sphere-like g-C3N4/BiOI Photocatalysts via a Reactable Ionic Liquid for Visible-Light-Driven Photocatalytic Degradation of Pollutants. J. Mater. Chem. A 2014, 2, 5340-5351. [CrossRef]

106. Liu, C.; Huang, H.; Du, X.; Zhang, T.; Tian, N.; Guo, Y.; Zhang, Y. In Situ Co-Crystallization for Fabrication of g-C3N4/Bi5O7I Heterojunction for Enhanced Visible-Light Photocatalysis. J. Phys. Chem. C 2015, 119, 17156-17165. [CrossRef]

107. Geng, X.; Chen, S.; Lv, X.; Jiang, W.; Wang, T. Synthesis of G-C3N4/Bi5O7I Microspheres with Enhanced Photocatalytic Activity under Visible Light. Appl. Surf. Sci. 2018, 462, 18-28. [CrossRef]

108. Zhou, M.; Wu, J.; Wang, H.; Guan, D.; Dong, X.; Wang, J.; Jia, T.; Liu, Q. Fabrication of Z-Scheme Heterojunction g-C3N4/Yb3+Bi5O7I Photocatalysts with Enhanced Photocatalytic Performance under Visible Irradiation for Hg0Removal. Energy Fuels 2020, 34, 16445-16455. [CrossRef]

109. Zhang, Z.; Pan, Z.; Guo, Y.; Wong, P.K.; Zhou, X.; Bai, R. In-Situ Growth of All-Solid Z-Scheme Heterojunction Photocatalyst of Bi7O9I3/g-C3N4 and High Efficient Degradation of Antibiotic under Visible Light. Appl. Catal. B Environ. 2020, $261,118212$. [CrossRef]

110. Feng, Z.; Zeng, L.; Zhang, Q.; Ge, S.; Zhao, X.; Lin, H.; He, Y. In Situ Preparation of G-C3N4/Bi4O5I2 Complex and Its Elevated Photoactivity in Methyl Orange Degradation under Visible Light. J. Environ. Sci. 2020, 87, 149-162. [CrossRef] [PubMed]

111. Zhang, F.; Wang, L.; Xiao, M.; Liu, F.; Xu, X.; Du, E. Construction of Direct Solid-State Z-Scheme g-C3N4/BiOI with Improved Photocatalytic Activity for Microcystin-LR Degradation. J. Mater. Res. 2018, 33, 201-212. [CrossRef]

112. Tian, N.; Huang, H.; Wang, S.; Zhang, T.; Du, X.; Zhang, Y. Facet-Charge-Induced Coupling Dependent Interfacial Photocharge Separation: A Case of BiOI/g-C3N4 p-n Junction. Appl. Catal. B Environ. 2020, 267, 118697. [CrossRef]

113. He, R.; Cheng, K.; Wei, Z.; Zhang, S.; Xu, D. Room-Temperature in Situ Fabrication and Enhanced Photocatalytic Activity of Direct Z-Scheme BiOI/g-C3N4 Photocatalyst. Appl. Surf. Sci. 2019, 465, 964-972. [CrossRef]

114. An, H.; Lin, B.; Xue, C.; Yan, X.; Dai, Y.; Wei, J.; Yang, G. Formation of BiOI/g-C3N4 Nanosheet Composites with High Visible-Light-Driven Photocatalytic Activity. Chin. J. Catal. 2018, 39, 654-663. [CrossRef]

115. Zhang, J.; Fu, J.; Wang, Z.; Cheng, B.; Dai, K.; Ho, W. Direct Z-Scheme Porous g-C3N4/BiOI Heterojunction for Enhanced Visible-Light Photocatalytic Activity. J. Alloys Compd. 2018, 766, 841-850. [CrossRef] 
116. Jiang, J.; Mu, Z.; Zhao, P.; Wang, H.; Lin, Y. Photogenerated Charge Behavior of BiOI/g-C3N4 Photocatalyst in Photoreduction of Cr (VI): A Novel Understanding for High-Performance. Mater. Chem. Phys. 2020, 252, 123194. [CrossRef]

117. Liu, W.; Qiao, L.; Zhu, A.; Liu, Y.; Pan, J. Constructing 2D BiOCl/C3N4 Layered Composite with Large Contact Surface for Visible-Light-Driven Photocatalytic Degradation. Appl. Surf. Sci. 2017, 426, 897-905. [CrossRef]

118. Wang, Q.; Wang, W.; Zhong, L.; Liu, D.; Cao, X.; Cui, F. Oxygen Vacancy-Rich 2D/2D BiOCl-g-C3N4 Ultrathin Heterostructure Nanosheets for Enhanced Visible-Light-Driven Photocatalytic Activity in Environmental Remediation. Appl. Catal. B Environ. 2018, 220, 290-302. [CrossRef]

119. Song, L.; Pang, Y.; Zheng, Y.; Ge, L. Hydrothermal Synthesis of Novel G-C3N4/BiOCl Heterostructure Nanodiscs for Efficient Visible Light Photodegradation of Rhodamine B. Appl. Phys. A 2017, 123, 500. [CrossRef]

120. Zhang, X.; An, D.; Feng, D.; Liang, F.; Chen, Z.; Liu, W.; Yang, Z.; Xian, M. In Situ Surfactant-Free Synthesis of Ultrathin $\mathrm{BiOCl} / \mathrm{g}-\mathrm{C} 3 \mathrm{~N} 4$ Nanosheets for Enhanced Visible-Light Photodegradation of Rhodamine B. Appl. Surf. Sci. 2019, 476, 706-715. [CrossRef]

121. Hou, W.; Deng, C.; Xu, H.; Li, D.; Zou, Z.; Xia, H.; Xia, D. N-p BiOCl@g-C $3 \mathrm{~N}_{4}$ Heterostructure with Rich-oxygen Vacancies for Photodegradation of Carbamazepine. ChemistrySelect 2020, 5, 2767-2777. [CrossRef]

122. Al Marzouqi, F.; Al Farsi, B.; Kuvarega, A.T.; Al Lawati, H.A.J.; Al Kindy, S.M.Z.; Kim, Y.; Selvaraj, R. Controlled MicrowaveAssisted Synthesis of the 2D-BiOCl/2D-g-C3 N4 Heterostructure for the Degradation of Amine-Based Pharmaceuticals under Solar Light Illumination. ACS Omega 2019, 4, 4671-4678. [CrossRef] [PubMed]

123. Liu, C.; Wu, Q.; Ji, M.; Zhu, H.; Hou, H.; Yang, Q.; Jiang, C.; Wang, J.; Tian, L.; Chen, J.; et al. Constructing Z-Scheme Charge Separation in 2D Layered Porous BiOBr/Graphitic C3N4 Nanosheets Nanojunction with Enhanced Photocatalytic Activity. J. Alloys Compd. 2017, 723, 1121-1131. [CrossRef]

124. Wu, J.; Xie, Y.; Ling, Y.; Dong, Y.; Li, J.; Li, S.; Zhao, J. Synthesis of Flower-Like g-C3N4/BiOBr and Enhancement of the Activity for the Degradation of Bisphenol A Under Visible Light Irradiation. Front. Chem. 2019, 7, 649. [CrossRef] [PubMed]

125. Jiang, M.; Shi, Y.; Huang, J.; Wang, L.; She, H.; Tong, J.; Su, B.; Wang, Q. Synthesis of Flowerlike G-C3N4/BiOBr with Enhanced Visible Light Photocatalytic Activity for Dye Degradation. Eur. J. Inorg. Chem. 2018, 2018, 1834-1841. [CrossRef]

126. Zhou, M.; Huang, W.; Zhao, Y.; Jin, Z.; Hua, X.; Li, K.; Tang, L.; Cai, Z. 2D G-C3N4/BiOBr Heterojunctions with Enhanced Visible Light Photocatalytic Activity. J. Nanoparticle Res. 2020, 22, 13. [CrossRef]

127. Lv, J.; Dai, K.; Zhang, J.; Liu, Q.; Liang, C.; Zhu, G. Facile Constructing Novel 2D Porous G-C3N4/BiOBr Hybrid with Enhanced Visible-Light-Driven Photocatalytic Activity. Sep. Purif. Technol. 2017, 178, 6-17. [CrossRef]

128. Kanagaraj, T.; Thiripuranthagan, S.; Paskalis, S.M.K.; Abe, H. Visible Light Photocatalytic Activities of Template Free Porous Graphitic Carbon Nitride-BiOBr Composite Catalysts towards the Mineralization of Reactive Dyes. Appl. Surf. Sci. 2017, 426, 1030-1045. [CrossRef]

129. Chen, B.; Zhou, L.; Tian, Y.; Yu, J.; Lei, J.; Wang, L.; Liu, Y.; Zhang, J. Z-Scheme Inverse Opal CN/BiOBr Photocatalysts for Highly Efficient Degradation of Antibiotics. Phys. Chem. Chem. Phys. 2019, 21, 12818-12825. [CrossRef]

130. Dong, Z.; Pan, J.; Wang, B.; Jiang, Z.; Zhao, C.; Wang, J.; Song, C.; Zheng, Y.; Cui, C.; Li, C. The P-n-Type Bi5O7I-Modified Porous C3N4 Nano-Heterojunction for Enhanced Visible Light Photocatalysis. J. Alloys Compd. 2018, 747, 788-795. [CrossRef]

131. Salimi, M.; Esrafili, A.; Sobhi, H.R.; Behbahani, M.; Gholami, M.; Farzadkia, M.; Jafari, A.J.; Kalantary, R.R. Photocatalytic Degradation of Metronidazole Using D-g- $\mathrm{C}_{3} \mathrm{~N}_{4}-\mathrm{Bi}_{5} \mathrm{O}_{7} \mathrm{I}$ Composites Under Visible Light Irradiation: Degradation Product, and Mechanisms. ChemistrySelect 2019, 4, 10288-10295. [CrossRef]

132. Tian, N.; Zhang, Y.; Liu, C.; Yu, S.; Li, M.; Huang, H. G-C3N4/Bi4O5I2 2D-2D Heterojunctional Nanosheets with Enhanced Visible-Light Photocatalytic Activity. RSC Adv. 2016, 6, 10895-10903. [CrossRef]

133. Ma, Y.; Chen, Y.; Feng, Z.; Zeng, L.; Chen, Q.; Jin, R.; Lu, Y.; Huang, Y.; Wu, Y.; He, Y. Preparation, Characterization of $\mathrm{Bi} 3 \mathrm{O} 4 \mathrm{Cl} / \mathrm{g}-\mathrm{C} 3 \mathrm{~N} 4$ Composite and Its Photocatalytic Activity in Dye Degradation. J. Water Process Eng. 2017, 18, 65-72. [CrossRef]

134. Zhao, J.; Ji, M.; Di, J.; Ge, Y.; Zhang, P.; Xia, J.; Li, H. Synthesis of G-C3N4/Bi4O5Br2 via Reactable Ionic Liquid and Its Cooperation Effect for the Enhanced Photocatalytic Behavior towards Ciprofloxacin Degradation. J. Photochem. Photobiol. A Chem. 2017, 347, 168-176. [CrossRef]

135. Yi, F.; Ma, J.; Lin, C.; Wang, L.; Zhang, H.; Qian, Y.; Zhang, K. Insights into the Enhanced Adsorption/Photocatalysis Mechanism of a Bi4O5Br2/g-C3N4 Nanosheet. J. Alloys Compd. 2020, 821, 153557. [CrossRef]

136. Jiang, J.; Song, Y.; Wang, X.; Li, T.; Li, M.; Lin, Y.; Xie, T.; Dong, S. Enhancing Aqueous Pollutant Photodegradation via a Fermi Level Matched Z-Scheme BiOI/Pt/g-C3N4 Photocatalyst: Unobstructed Photogenerated Charge Behavior and Degradation Pathway Exploration. Catal. Sci. Technol. 2020, 10, 3324-3333. [CrossRef]

137. Li, Z.; Jin, C.; Lv, C.; Wang, M.; Kang, J.; Liu, S.; Xie, Y.; Zhu, T. Construction of G-C3N4/Eu(III) Doped Bi24O31Cl10 Heterojunction for the Enhanced Visible-Light Photocatalytic Performance. Mater. Chem. Phys. 2019, 237, 121829. [CrossRef]

138. Wang, L.; Zhang, H.; Guo, C.; Feng, L.; Li, C.; Wang, W. Facile Constructing Plasmonic Z-Scheme Au NPs/g-C3N4/BiOBr for Enhanced Visible Light Photocatalytic Activity. J. Fuel Chem. Technol. 2019, 47, 834-842. [CrossRef]

139. Bai, Y.; Chen, T.; Wang, P.; Wang, L.; Ye, L.; Shi, X.; Bai, W. Size-Dependent Role of Gold in g-C3N4/BiOBr/Au System for Photocatalytic CO2 Reduction and Dye Degradation. Sol. Energy Mater. Sol. Cells 2016, 157, 406-414. [CrossRef]

140. Asadzadeh-Khaneghah, S.; Habibi-Yangjeh, A.; Seifzadeh, D. Graphitic Carbon Nitride Nanosheets Coupled with Carbon Dots and BiOI Nanoparticles: Boosting Visible-Light-Driven Photocatalytic Activity. J. Taiwan Inst. Chem. Eng. 2018, 87, 98-111. [CrossRef] 
141. Wang, Q.; Li, Y.; Huang, L.; Zhang, F.; Wang, H.; Wang, C.; Zhang, Y.; Xie, M.; Li, H. Enhanced Photocatalytic Degradation and Antibacterial Performance by GO/CN/BiOI Composites under LED Light. Appl. Surf. Sci. 2019, 497, 143753. [CrossRef]

142. You, Z.; Shen, Q.; Su, Y.; Yu, Y.; Wang, H.; Qin, T.; Zhang, F.; Cheng, D.; Yang, H. Construction of a Z-Scheme Core-Shell g-C3N4/MCNTs/BiOI Nanocomposite Semiconductor with Enhanced Visible-Light Photocatalytic Activity. New J. Chem. 2017, 42, 489-496. [CrossRef]

143. Hu, X.; Hu, J.; Peng, Q.; Ma, X.; Dong, S.; Wang, H. Construction of 2D All-Solid-State Z-Scheme g-C3N4/BiOI/RGO Hybrid Structure Immobilized on Ni Foam for CO2 Reduction and Pollutant Degradation. Mater. Res. Bull. 2020, 122, 110682. [CrossRef]

144. Bao, Y.; Chen, K. Novel Z-Scheme BiOBr/Reduced Graphene Oxide/Protonated g-C3N4 Photocatalyst: Synthesis, Characterization, Visible Light Photocatalytic Activity and Mechanism. Appl. Surf. Sci. 2018, 437, 51-61. [CrossRef]

145. Zhang, M.; Lai, C.; Li, B.; Huang, D.; Zeng, G.; Xu, P.; Qin, L.; Liu, S.; Liu, X.; Yi, H.; et al. Rational Design 2D/2D BiOBr/CDs/gC3N4 Z-Scheme Heterojunction Photocatalyst with Carbon Dots as Solid-State Electron Mediators for Enhanced Visible and NIR Photocatalytic Activity: Kinetics, Intermediates, and Mechanism Insight. J. Catal. 2019, 369, 469-481. [CrossRef]

146. Yu, X.; Wu, P.; Qi, C.; Shi, J.; Feng, L.; Li, C.; Wang, L. Ternary-Component Reduced Graphene Oxide Aerogel Constructed by g-C3N4/BiOBr Heterojunction and Graphene Oxide with Enhanced Photocatalytic Performance. J. Alloys Compd. 2017, 729, 162-170. [CrossRef]

147. Shi, Z.; Zhang, Y.; Shen, X.; Duoerkun, G.; Zhu, B.; Zhang, L.; Li, M.; Chen, Z. Fabrication of G-C3N4/BiOBr Heterojunctions on Carbon Fibers as Weaveable Photocatalyst for Degrading Tetracycline Hydrochloride under Visible Light. Chem. Eng. J. 2020, 386, 124010. [CrossRef]

148. Chen, Y.; Ji, X.; Vadivel, S.; Paul, B. Anchoring Carbon Spheres on BiOBr/g-C3N4 Matrix for High-Performance Visible Light Photocatalysis. Ceram. Int. 2018, 44, 23320-23323. [CrossRef]

149. Li, J.; Yu, X.; Zhu, Y.; Fu, X.; Zhang, Y. 3D-2D-3D BiOI/Porous g-C3N4/Graphene Hydrogel Composite Photocatalyst with Synergy of Adsorption-Photocatalysis in Static and Flow Systems. J. Alloys Compd. 2021, 850, 156778. [CrossRef]

150. Zhou, X.; Shao, C.; Yang, S.; Li, X.; Guo, X.; Wang, X.; Li, X.; Liu, Y. Heterojunction of G-C3N4/BiOI Immobilized on Flexible Electrospun Polyacrylonitrile Nanofibers: Facile Preparation and Enhanced Visible Photocatalytic Activity for Floating Photocatalysis. Acs Sustain. Chem. Eng. 2018, 6, 2316-2323. [CrossRef]

151. Wang, X.; Zhou, X.; Shao, C.; Li, X.; Liu, Y. Graphitic Carbon Nitride/BiOI Loaded on Electrospun Silica Nanofibers with Enhanced Photocatalytic Activity. Appl. Surf. Sci. 2018, 455, 952-962. [CrossRef]

152. Huang, Y.; Zhang, X.; Zhang, K.; Lu, P.; Zhang, D. Facile Fabrication of Sandwich-like BiOI/AgI/g-C3N4 Composites for Efficient Photocatalytic Degradation of Methyl Orange and Reduction of Cr(VI). J. Nanoparticle Res. 2018, 20, 328. [CrossRef]

153. Jiang, X.; Lai, S.; Xu, W.; Fang, J.; Chen, X.; Beiyuan, J.; Zhou, X.; Lin, K.; Liu, J.; Guan, G. Novel Ternary BiOI/g-C3N4/CeO2 Catalysts for Enhanced Photocatalytic Degradation of Tetracycline under Visible-Light Radiation via Double Charge Transfer Process. J. Alloys Compd. 2019, 809, 151804. [CrossRef]

154. Gholizadeh Khasevani, S.; Gholami, M.R. Engineering a Highly Dispersed Core@shell Structure for Efficient Photocatalysis: A Case Study of Ternary Novel BiOI@MIL-88A(Fe)@g-C3N4 Nanocomposite. Mater. Res. Bull. 2018, 106, 93-102. [CrossRef]

155. Kang, J.; Jin, C.; Li, Z.; Wang, M.; Chen, Z.; Wang, Y. Dual Z-Scheme MoS2/g-C3N4/Bi24O31Cl10 Ternary Heterojunction Photocatalysts for Enhanced Visible-Light Photodegradation of Antibiotic. J. Alloys Compd. 2020, 825, 153975. [CrossRef]

156. Zhao, W.; Wang, A.; Wang, Y.; Lv, C.; Zhu, W.; Dou, S.; Wang, Q.; Zhong, Q. Accessible Fabrication and Mechanism Insight of Heterostructured BiOCl/Bi2MoO6/g-C3N4 Nanocomposites with Efficient Photosensitized Activity. J. Alloys Compd. 2017, 726, 164-172. [CrossRef]

157. Bellamkonda, S.; Ranga Rao, G. Nanojunction-Mediated Visible Light Photocatalytic Enhancement in Heterostructured Ternary $\mathrm{BiOCl} / \mathrm{CdS} / \mathrm{g}-\mathrm{C} 3 \mathrm{~N} 4$ Nanocomposites. Catal. Today 2019, 321-322, 18-25. [CrossRef]

158. Dong, X.; Sun, Z.; Zhang, X.; Li, C.; Zheng, S. Construction of BiOCl/g-C3N4/Kaolinite Composite and Its Enhanced Photocatalysis Performance under Visible-Light Irradiation. J. Taiwan Inst. Chem. Eng. 2018, 84, 203-211. [CrossRef]

159. He, B.; Du, Y.; Feng, Y.; Du, M.; Wang, J.; Qu, J.; Liu, Y.; Jiang, N.; Wang, J.; Sun, X. Fabrication of Novel Ternary Direct Z-Scheme + isotype Heterojunction Photocatalyst g-C3N4/g-C3N4/BiOBr with Enhanced Photocatalytic Performance. Appl. Surf. Sci. 2020, 506, 145031. [CrossRef]

160. Tang, G.; Zhang, F.; Huo, P.; Zulfiqarc, S.; Xu, J.; Yan, Y.; Tang, H. Constructing Novel Visible-Light-Driven Ternary Photocatalyst of AgBr Nanoparticles Decorated 2D/2D Heterojunction of g-C3N4/BiOBr Nanosheets with Remarkably Enhanced Photocatalytic Activity for Water-Treatment. Ceram. Int. 2019, 45, 19197-19205. [CrossRef]

161. Liu, N.; Xie, H.; Li, J.; Zhao, Y.; Wang, N. Synthesis and High Visible Light Photocatalytic Activity of Ternary Brookite-g-C3N4BiOBr Composite. Nano 2020, 15, 2050045. [CrossRef]

162. Zhong, S.; Zhou, H.; Shen, M.; Yao, Y.; Gao, Q. Rationally Designed a G-C3N4/BiOI/Bi2O2CO3 Composite with Promoted Photocatalytic Activity. J. Alloys Compd. 2021, 853, 157307. [CrossRef]

163. Chou, S.-Y.; Chen, C.-C.; Dai, Y.-M.; Lin, J.-H.; Lee, W.W. Novel Synthesis of Bismuth Oxyiodide/Graphitic Carbon Nitride Nanocomposites with Enhanced Visible-Light Photocatalytic Activity. RSC Adv. 2016, 6, 33478-33491. [CrossRef]

164. Liu, B.; Han, X.; Wang, Y.; Fan, X.; Wang, Z.; Zhang, J.; Shi, H. Synthesis of G-C3N4/BiOI/BiOBr Heterostructures for Efficient Visible-Light-Induced Photocatalytic and Antibacterial Activity. J. Mater. Sci. Mater. Electron. 2018, 29, 14300-14310. [CrossRef]

165. Zhang, W.; Liang, Y. Facile Synthesis of Ternary G-C3N4@BiOCl/Bi12O17Cl2 Composites with Excellent Visible Light Photocatalytic Activity for NO Removal. Front. Chem. 2019, 7, 231. [CrossRef] 
166. Kumar, A.; Kumar, A.; Sharma, G.; Al-Muhtaseb, A.H.; Naushad, M.U.; Ghfar, A.A.; Stadler, F.J. Quaternary Magnetic BiOCl/gC3N4/Cu2O/Fe3O4 Nano-Junction for Visible Light and Solar Powered Degradation of Sulfamethoxazole from Aqueous Environment. Chem. Eng. J. 2018, 334, 462-478. [CrossRef]

167. Yuan, D.; Huang, L.; Li, Y.; Xu, Y.; Xu, H.; Huang, S.; Yan, J.; He, M.; Li, H. Synthesis and Photocatalytic Activity of GC3N4/BiOI/BiOBr Ternary Composites. RSC Adv. 2016, 6, 41204-41213. [CrossRef]

168. Qu, J.; Du, Y.; Feng, Y.; Wang, J.; He, B.; Du, M.; Liu, Y.; Jiang, N. Visible-Light-Responsive K-Doped g-C3N4/BiOBr Hybrid Photocatalyst with Highly Efficient Degradation of Rhodamine B and Tetracycline. Mater. Sci. Semicond. Process. 2020, 112, 105023. [CrossRef]

169. Guo, F.; Chen, J.; Zhao, J.; Chen, Z.; Xia, D.; Zhan, Z.; Wang, Q. Z-Scheme Heterojunction g-C3N4@PDA/BiOBr with Biomimetic Polydopamine as Electron Transfer Mediators for Enhanced Visible-Light Driven Degradation of Sulfamethoxazole. Chem. Eng. J. 2020, 386, 124014. [CrossRef]

170. Li, H.; Ai, Z.; Zhang, L. Surface Structure-Dependent Photocatalytic O2 Activation for Pollutant Removal with Bismuth Oxyhalides. Chem. Commun. 2020, 56, 15282. [CrossRef]

171. Ye, L.; Jin, X.; Ji, X.; Liu, C.; Su, Y.; Xie, H.; Liu, C. Facet-Dependent Photocatalytic Reduction of CO2 on BiOI Nanosheets. Chem. Eng. J. 2016, 291, 39-46. [CrossRef]

172. Ye, L.; Wang, H.; Jin, X.; Su, Y.; Wang, D.; Xie, H.; Liu, X.; Liu, X. Synthesis of Olive-Green Few-Layered BiOI for Efficient Photoreduction of CO2 into Solar Fuels under Visible/near-Infrared Light. Sol. Energy Mater. Sol. Cells 2016, 144, 732-739. [CrossRef]

173. Yu, H.; Huang, H.; Xu, K.; Hao, W.; Guo, Y.; Wang, S.; Shen, X.; Pan, S.; Zhang, Y. Liquid-Phase Exfoliation into Monolayered BiOBr Nanosheets for Photocatalytic Oxidation and Reduction. ACS Sustain. Chem. Eng. 2017, 5, 10499-10508. [CrossRef]

174. Kong, X.Y.; Ng, B.J.; Tan, K.H.; Chen, X.; Wang, H.; Mohamed, A.R.; Chai, S.P. Simultaneous Generation of Oxygen Vacancies on Ultrathin BiOBr Nanosheets during Visible-Light-Driven CO2 Photoreduction Evoked Superior Activity and Long-Term Stability. Catal. Today 2018, 314, 20-27. [CrossRef]

175. Kong, X.Y.; Lee, W.P.C.; Ong, W.-J.; Chai, S.-P.; Mohamed, A.R. Oxygen-Deficient BiOBr as a Highly Stable Photocatalyst for Efficient $\mathrm{CO}_{2}$ Reduction into Renewable Carbon-Neutral Fuels. ChemCatChem 2016, 8, 3074-3081. [CrossRef]

176. Gao, M.; Yang, J.; Sun, T.; Zhang, Z.; Zhang, D.; Huang, H.; Lin, H.; Fang, Y.; Wang, X. Persian Buttercup-like BiOBrxCl1-x Solid Solution for Photocatalytic Overall CO2 Reduction to CO and O2. Appl. Catal. B Environ. 2019, 243, 734-740. [CrossRef]

177. Jin, J.; Wang, Y.; He, T. Preparation of Thickness-Tunable BiOCl Nanosheets with High Photocatalytic Activity for Photoreduction of CO2. RSC Adv. 2015, 5, 100244-100250. [CrossRef]

178. Ma, Z.; Li, P.; Ye, L.; Zhou, Y.; Su, F.; Ding, C.; Xie, H.; Bai, Y.; Wong, P.K. Oxygen Vacancies Induced Exciton Dissociation of Flexible BiOCl Nanosheets for Effective Photocatalytic CO2 Conversion. J. Mater. Chem. A 2017, 5, 24995-25004. [CrossRef]

179. Zhang, L.; Wang, W.; Jiang, D.; Gao, E.; Sun, S. Photoreduction of CO2 on BiOCl Nanoplates with the Assistance of Photoinduced Oxygen Vacancies. Nano Res. 2015, 8, 821-831. [CrossRef]

180. Bai, Y.; Yang, P.; Wang, P.; Xie, H.; Dang, H.; Ye, L. Semimetal Bismuth Mediated UV-Vis-IR Driven Photo-Thermocatalysis of Bi4O5I2 for Carbon Dioxide to Chemical Energy. J. Co2 Util. 2018, 23, 51-60. [CrossRef]

181. Ye, L.; Jin, X.; Liu, C.; Ding, C.; Xie, H.; Chu, K.H.; Wong, P.K. Thickness-Ultrathin and Bismuth-Rich Strategies for BiOBr to Enhance Photoreduction of $\mathrm{CO}_{2}$ into Solar Fuels. Appl. Catal. B Environ. 2016, 187, 281-290. [CrossRef]

182. Bai, Y.; Yang, P.; Wang, L.; Yang, B.; Xie, H.; Zhou, Y.; Ye, L. Ultrathin Bi4O5Br2 Nanosheets for Selective Photocatalytic CO2 Conversion into CO. Chem. Eng. J. 2019, 360, 473-482. [CrossRef]

183. Ding, C.; Ye, L.; Zhao, Q.; Zhong, Z.; Liu, K.; Xie, H.; Bao, K.; Zhang, X.; Huang, Z. Synthesis of BixOyIz from Molecular Precursor and Selective Photoreduction of $\mathrm{CO}_{2}$ into CO. J. Co2 Util. 2016, 14, 135-142. [CrossRef]

184. Bai, Y.; Ye, L.; Wang, L.; Shi, X.; Wang, P.; Bai, W.; Wong, P.K. G-C $\mathrm{C}_{3} \mathrm{~N}_{4} / \mathrm{Bi}_{4} \mathrm{O}_{5} \mathrm{I}_{2}$ Heterojunction with I3-/I- Redox Mediator for Enhanced Photocatalytic CO2 Conversion. Appl. Catal. B Environ. 2016, 194, 98-104. [CrossRef]

185. Wang, J.-C.; Yao, H.-C.; Fan, Z.-Y.; Zhang, L.; Wang, J.-S.; Zang, S.-Q.; Li, Z.-J. Indirect Z-Scheme BiOI/g-C $3 \mathrm{~N}_{4}$ Photocatalysts with Enhanced Photoreduction CO2 Activity under Visible Light Irradiation. ACS Appl. Mater. Interfaces 2016, 8, 3765-3775. [CrossRef] [PubMed]

186. Chen, Y.; Wang, F.; Cao, Y.; Zhang, F.; Zou, Y.; Huang, Z.; Ye, L.; Zhou, Y. Interfacial Oxygen Vacancy Engineered Two-Dimensional g-C3N4/BiOCl Heterostructures with Boosted Photocatalytic Conversion of CO2. ACS Appl. Energy Mater. 2020. [CrossRef]

187. Ayyub, M.M.; Singh, R.; Rao, C.N.R. Hydrogen Generation by Solar Water Splitting Using 2D Nanomaterials. Sol. Rrl 2020, 4 , 2000050. [CrossRef]

188. Pan, H.X.; Feng, L.P.; Zeng, W.; Zhang, Q.C.; Zhang, X.D.; Liu, Z.T. Active Sites in Single-Layer BiOX (X = Cl, Br, and I) Catalysts for the Hydrogen Evolution Reaction. Inorg. Chem. 2019, 58, 13195-13202. [CrossRef]

189. Fang, W.; Shangguan, W. A Review on Bismuth-Based Composite Oxides for Photocatalytic Hydrogen Generation. Int. J. Hydrog. Energy 2019, 44, 895-912. [CrossRef]

190. Jin, X.; Ye, L.; Xie, H.; Chen, G. Bismuth-Rich Bismuth Oxyhalides for Environmental and Energy Photocatalysis. Coord. Chem. Rev. 2017, 349, 84-101. [CrossRef]

191. Ye, L.; Jin, X.; Leng, Y.; Su, Y.; Xie, H.; Liu, C. Synthesis of Black Ultrathin BiOCl Nanosheets for Efficient Photocatalytic H2 Production under Visible Light Irradiation. J. Power Sources 2015, 293, 409-415. [CrossRef] 
192. Li, M.; Yu, S.; Huang, H.; Li, X.; Feng, Y.; Wang, C.; Wang, Y.; Ma, T.; Guo, L.; Zhang, Y. Unprecedented Eighteen-Faceted BiOCl with a Ternary Facet Junction Boosting Cascade Charge Flow and Photo-redox. Angew. Chem. Int. Ed. 2019, 58, 9517-9521. [CrossRef]

193. Lee, G.J.; Zheng, Y.C.; Wu, J.J. Fabrication of Hierarchical Bismuth Oxyhalides (BiOX, X = Cl, Br, I) Materials and Application of Photocatalytic Hydrogen Production from Water Splitting. Catal. Today 2018, 307, 197-204. [CrossRef]

194. Li, J.; Zhan, G.; Yu, Y.; Zhang, L. Superior Visible Light Hydrogen Evolution of Janus Bilayer Junctions via Atomic-Level Charge Flow Steering. Nat. Commun. 2016, 7, 11480. [CrossRef] [PubMed]

195. Li, Y.F.; Liu, Z.P.; Liu, L.; Gao, W. Mechanism and Activity of Photocatalytic Oxygen Evolution on Titania Anatase in Aqueous Surroundings. J. Am. Chem. Soc. 2010, 132, 13008-13015. [CrossRef]

196. Di, J.; Chen, C.; Yang, S.Z.; Ji, M.; Yan, C.; Gu, K.; Xia, J.; Li, H.; Li, S.; Liu, Z. Defect Engineering in Atomically-Thin Bismuth Oxychloride towards Photocatalytic Oxygen Evolution. J. Mater. Chem. A 2017, 5, 14144-14151. [CrossRef]

197. Bai, L.; Ye, F.; Li, L.; Lu, J.; Zhong, S.; Bai, S. Facet Engineered Interface Design of Plasmonic Metal and Cocatalyst on BiOCl Nanoplates for Enhanced Visible Photocatalytic Oxygen Evolution. Small 2017, 13, 1701607. [CrossRef] [PubMed]

198. Cui, D.; Wang, L.; Xu, K.; Ren, L.; Weng, L.; Yu, Y.; Du, Y.; Hao, W. Band-Gap Engineering of BiOCl with Oxygen Vacancies for Efficient Photooxidation Properties under Visible-Light Irradiation. J. Mater. Chem. A 2018, 6, 2193-2199. [CrossRef]

199. Ji, M.; Chen, R.; Di, J.; Liu, Y.; Li, K.; Chen, Z.; Xia, J.; Li, H. Oxygen Vacancies Modulated Bi-Rich Bismuth Oxyiodide Microspheres with Tunable Valence Band Position to Boost the Photocatalytic Activity. J. Colloid Interface Sci. 2019, 533, 612-620. [CrossRef]

200. Xiong, X.; Zhou, T.; Liu, X.; Ding, S.; Hu, J. Surfactant-Mediated Synthesis of Single-Crystalline Bi3O4Br Nanorings with Enhanced Photocatalytic Activity. J. Mater. Chem. A 2017, 5, 15706-15713. [CrossRef]

201. Ning, S.; Shi, X.; Zhang, H.; Lin, H.; Zhang, Z.; Long, J.; Li, Y.; Wang, X. Reconstructing Dual-Induced $\{001\}$ Facets Bismuth Oxychloride Nanosheets Heterostructures: An Effective Strategy to Promote Photocatalytic Oxygen Evolution. Sol. RRL 2019, 3, 1900059. [CrossRef]

202. Dong, G.; Jacobs, D.L.; Zang, L.; Wang, C. Carbon Vacancy Regulated Photoreduction of NO to N2 over Ultrathin G-C3N4 Nanosheets. Appl. Catal. B Environ. 2017, 218, 515-524. [CrossRef]

203. Ye, C.; Li, J.X.; Li, Z.J.; Li, X.B.; Fan, X.B.; Zhang, L.P.; Chen, B.; Tung, C.H.; Wu, L.Z. Enhanced Driving Force and Charge Separation Efficiency of Protonated G-C3N4 for Photocatalytic O2 Evolution. ACS Catal. 2015, 5, 6973-6979. [CrossRef]

204. Zhang, J.; Grzelczak, M.; Hou, Y.; Maeda, K.; Domen, K.; Fu, X.; Antonietti, M.; Wang, X. Photocatalytic Oxidation of Water by Polymeric Carbon Nitride Nanohybrids Made of Sustainable Elements. Chem. Sci. 2012, 3, 443-446. [CrossRef]

205. Li, H.; Shang, J.; Ai, Z.; Zhang, L. Efficient Visible Light Nitrogen Fixation with BiOBr Nanosheets of Oxygen Vacancies on the Exposed \{001\} Facets. J. Am. Chem. Soc. 2015, 137, 6393-6399. [CrossRef] [PubMed]

206. Li, H.; Shang, J.; Shi, J.; Zhao, K.; Zhang, L. Facet-Dependent Solar Ammonia Synthesis of BiOCl Nanosheets via a Proton-Assisted Electron Transfer Pathway. Nanoscale 2016, 8, 1986-1993. [CrossRef]

207. Bai, Y.; Ye, L.; Chen, T.; Wang, L.; Shi, X.; Zhang, X.; Chen, D. Facet-Dependent Photocatalytic N2 Fixation of Bismuth-Rich Bi5O7I Nanosheets. Acs Appl. Mater. Interfaces 2016, 8, 27661-27668. [CrossRef]

208. Zhang, N.; Li, L.; Shao, Q.; Zhu, T.; Huang, X.; Xiao, X. Fe-Doped BiOCl Nanosheets with Light-Switchable Oxygen Vacancies for Photocatalytic Nitrogen Fixation. Acs Appl. Energy Mater. 2019, 2, 8394-8398. [CrossRef]

209. Liu, Y.; Hu, Z.; Yu, J.C. Fe Enhanced Visible-Light-Driven Nitrogen Fixation on Biobr Nanosheets. Chem. Mater. 2020, 32, 1488-1494. [CrossRef]

210. Cao, S.; Fan, B.; Feng, Y.; Chen, H.; Jiang, F.; Wang, X. Sulfur-Doped g-C3N4 Nanosheets with Carbon Vacancies: General Synthesis and Improved Activity for Simulated Solar-Light Photocatalytic Nitrogen Fixation. Chem. Eng. J. 2018, 353, 147-156. [CrossRef]

211. Xue, Y.; Guo, Y.; Liang, Z.; Cui, H.; Tian, J. Porous G-C3N4 with Nitrogen Defects and Cyano Groups for Excellent Photocatalytic Nitrogen Fixation without Co-Catalysts. J. Colloid Interface Sci. 2019, 556, 206-213. [CrossRef]

212. Xiao, X.; Jiang, J.; Zhang, L. Selective Oxidation of Benzyl Alcohol into Benzaldehyde over Semiconductors under Visible Light: The Case of Bi12O17Cl2 Nanobelts. Appl. Catal. B Environ. 2013, 142-143, 487-493. [CrossRef]

213. Han, A.; Zhang, H.; Chuah, G.K.; Jaenicke, S. Influence of the Halide and Exposed Facets on the Visible-Light Photoactivity of Bismuth Oxyhalides for Selective Aerobic Oxidation of Primary Amines. Appl. Catal. B Environ. 2017, 219, 269-275. [CrossRef]

214. Wang, H.; Yong, D.; Chen, S.; Jiang, S.; Zhang, X.; Shao, W.; Zhang, Q.; Yan, W.; Pan, B.; Xie, Y. Oxygen-Vacancy-Mediated Exciton Dissociation in Biobr for Boosting Charge-Carrier-Involved Molecular Oxygen Activation. J. Am. Chem. Soc. 2018, 140, 1760-1766. [CrossRef] [PubMed]

215. Wu, Y.; Yuan, B.; Li, M.; Zhang, W.H.; Liu, Y.; Li, C. Well-Defined BiOCl Colloidal Ultrathin Nanosheets: Synthesis, Characterization, and Application in Photocatalytic Aerobic Oxidation of Secondary Amines. Chem. Sci. 2015, 6, 1873-1878. [CrossRef] [PubMed]

216. Dai, Y.; Ren, P.; Li, Y.; Lv, D.; Shen, Y.; Li, Y.; Niemantsverdriet, H.; Besenbacher, F.; Xiang, H.; Hao, W.; et al. Solid Base Bi24O31Br10 $(\mathrm{OH}) \delta$ with Active Lattice Oxygen for the Efficient Photo-Oxidation of Primary Alcohols to Aldehydes. Angew. Chem. Int. Ed. 2019, 58, 6265-6270. [CrossRef]

217. Juntrapirom, S.; Anuchai, S.; Thongsook, O.; Pornsuwan, S.; Meepowpan, P.; Thavornyutikarn, P.; Phanichphant, S.; Tantraviwat, D.; Inceesungvorn, B. Photocatalytic Activity Enhancement of G-C3N4/BiOBr in Selective Transformation of Primary Amines to Imines and Its Reaction Mechanism. Chem. Eng. J. 2020, 394, 124934. [CrossRef] 
218. Liu, D.; Chen, D.; Li, N.; Xu, Q.; Li, H.; He, J.; Lu, J. Surface Engineering of G-C3N4 by Stacked BiOBr Sheets Rich in Oxygen Vacancies for Boosting Photocatalytic Performance. Angew. Chem. Int. Ed. 2020, 59, 4519-4524. [CrossRef]

219. Ye, L.; Zan, L.; Tian, L.; Peng, T.; Zhang, J. The $\{001\}$ Facets-Dependent High Photoactivity of BiOCl Nanosheets. Chem. Commun. 2011, 47, 6951-6953. [CrossRef]

220. Jiang, J.; Zhao, K.; Xiao, X.; Zhang, L. Synthesis and Facet-Dependent Photoreactivity of BiOCl Single-Crystalline Nanosheets. J. Am. Chem. Soc. 2012, 134, 4473-4476. [CrossRef]

221. Zhao, Y.; Yu, T.; Tan, X.; Xie, C.; Wang, S. SDS-Assisted Solvothermal Synthesis of Rose-like BiOBr Partially Enclosed by $\{111\}$ Facets and Enhanced Visible-Light Photocatalytic Activity. Dalton Trans. 2015, 44, 20475-20483. [CrossRef]

222. Peng, Y.; Xu, J.; Liu, T.; Mao, Y.G. Controlled Synthesis of One-Dimensional BiOBr with Exposed (110) Facets and Enhanced Photocatalytic Activity. CrystEngComm 2017, 19, 6473-6480. [CrossRef]

223. Ye, L.; Deng, K.; Xu, F.; Tian, L.; Peng, T.; Zan, L. Increasing Visible-Light Absorption for Photocatalysis with Black BiOCl. Phys. Chem. Chem. Phys. 2012, 14, 82-85. [CrossRef]

224. Wang, X.J.; Zhao, Y.; Li, F.T.; Dou, L.J.; Li, Y.P.; Zhao, J.; Hao, Y.J. A Chelation Strategy for In-Situ Constructing Surface Oxygen Vacancy on $\{001\}$ Facets Exposed BiOBr Nanosheets. Sci. Rep. 2016, 6, 1-11. [CrossRef] [PubMed]

225. Wu, J.; Li, X.; Shi, W.; Ling, P.; Sun, Y.; Jiao, X.; Gao, S.; Liang, L.; Xu, J.; Yan, W.; et al. Efficient Visible-Light-Driven $\mathrm{CO}_{2}$ Reduction Mediated by Defect-Engineered BiOBr Atomic Layers. Angew. Chem. Int. Ed. 2018, 57, 8719-8723. [CrossRef] [PubMed]

226. Hu, J.; Zhai, C.; Gao, H.; Zeng, L.; Du, Y.; Zhu, M. Enhanced Photo-Assisted Ethanol Electro-Oxidation Activity by Using Broadband Visible Light Absorption of a Graphitic C3 N4 /BiOI Carrier. Sustain. Energy Fuels 2019, 3, 439-449. [CrossRef]

227. Wang, K.; Li, J.; Zhang, G. Ag-Bridged Z-Scheme 2D/2D Bi5FeTi3O15/g-C3N4 Heterojunction for Enhanced Photocatalysis: Mediator-Induced Interfacial Charge Transfer and Mechanism Insights. Acs Appl. Mater. Interfaces 2019, 11, $27686-27696$. [CrossRef] [PubMed]

228. Zhang, J.; Wang, Z.; Fan, M.; Tong, P.; Sun, J.; Dong, S.; Sun, J. Ultra-Light and Compressible 3D BiOCl/RGO Aerogel with Enriched Synergistic Effect of Adsorption and Photocatalytic Degradation of Oxytetracycline. J. Mater. Res. Technol. 2019, 8, 4577-4587. [CrossRef]

229. Di, J.; Xia, J.; Ji, M.; Wang, B.; Yin, S.; Zhang, Q.; Chen, Z.; Li, H. Carbon Quantum Dots Modified BiOCl Ultrathin Nanosheets with Enhanced Molecular Oxygen Activation Ability for Broad Spectrum Photocatalytic Properties and Mechanism Insight. Acs Appl. Mater. Interfaces 2015, 7, 20111-20123. [CrossRef] [PubMed]

230. Huang, J.; Chen, W.; Yu, X.; Fu, X.; Zhu, Y.; Zhang, Y. Fabrication of a Ternary BiOCl/CQDs/RGO Photocatalyst: The Roles of CQDs and RGO in Adsorption-Photocatalytic Removal of Ciprofloxacin. Colloids Surf. A Physicochem. Eng. Asp. 2020, 597, 124758. [CrossRef]

231. Yu, X.; Shi, J.; Feng, L.; Li, C.; Wang, L. A Three-Dimensional BiOBr/RGO Heterostructural Aerogel with Enhanced and Selective Photocatalytic Properties under Visible Light. Appl. Surf. Sci. 2017, 396, 1775-1782. [CrossRef]

232. Allagui, L.; Chouchene, B.; Gries, T.; Medjahdi, G.; Girot, E.; Framboisier, X.; Amara, A.B.H.; Balan, L.; Schneider, R. Core/Shell RGO/BiOBr Particles with Visible Photocatalytic Activity towards Water Pollutants. Appl. Surf. Sci. 2019, 490, 580-591. [CrossRef]

233. Xue, J.; Li, X.; Ma, S.; Xu, P.; Wang, M.; Ye, Z. Facile Fabrication of BiOCl/RGO/Protonated g-C3N4 Ternary Nanocomposite as Z-Scheme Photocatalyst for Tetracycline Degradation and Benzyl Alcohol Oxidation. J. Mater. Sci. 2019, 54, 1275-1290. [CrossRef]

234. Huang, H.; Liu, C.; Ou, H.; Ma, T.; Zhang, Y. Self-Sacrifice Transformation for Fabrication of Type-I and Type-II Heterojunctions in Hierarchical Bi x O y I z /g-C3 N4 for Efficient Visible-Light Photocatalysis. Appl. Surf. Sci. 2019, 470, 1101-1110. [CrossRef]

235. Low, J.; Jiang, C.; Cheng, B.; Wageh, S.; Al-Ghamdi, A.A.; Yu, J. A Review of Direct Z-Scheme Photocatalysts. Small Methods 2017, 1, 1700080. [CrossRef]

236. Zhou, P.; Yu, J.; Jaroniec, M. All-Solid-State Z-Scheme Photocatalytic Systems. Adv. Mater. 2014, 26, 4920-4935. [CrossRef]

237. Liang, Q.; Cui, S.; Jin, J.; Liu, C.; Xu, S.; Yao, C.; Li, Z. Fabrication of BiOI@UIO-66( $\left.\mathrm{NH}_{2}\right) @ g-C 3$ N4 Ternary Z-Scheme Heterojunction with Enhanced Visible-Light Photocatalytic Activity. Appl. Surf. Sci. 2018, 456, 899-907. [CrossRef]

238. Liang, S.; He, M.; Guo, J.; Yue, J.; Pu, X.; Ge, B.; Li, W. Fabrication and Characterization of BiOBr:Yb3+,Er3+/g-C3N4 p-n Junction Photocatalysts with Enhanced Visible-NIR-Light-Driven Photoactivities. Sep. Purif. Technol. 2018, 206, 69-79. [CrossRef]

239. Bai, Y.; Wang, P.Q.; Liu, J.Y.; Liu, X.J. Enhanced Photocatalytic Performance of Direct Z-Scheme BiOCl-g-C3N4 Photocatalysts. Rsc Adv. 2014, 4, 19456-19461. [CrossRef]

240. Zhao, W.; Wang, W.; Shi, H. 2D/2D Z-Scheme BiO1-XBr/g-C3N4 Heterojunction with Rich Oxygen Vacancies as Electron Mediator for Enhanced Visible-Light Degradation Activity. Appl. Surf. Sci. 2020, 528, 146925. [CrossRef]

241. Tang, Z.K.; Yin, W.J.; Zhang, L.; Wen, B.; Zhang, D.Y.; Liu, L.M.; Lau, W.M. Spatial Separation of Photo-Generated Electron-Hole Pairs in BiOBr/BiOI Bilayer to Facilitate Water Splitting. Sci. Rep. 2016, 6, 1-9. [CrossRef] [PubMed]

242. Tu, W.; Xu, Y.; Wang, J.; Zhang, B.; Zhou, T.; Yin, S.; Wu, S.; Li, C.; Huang, Y.; Zhou, Y.; et al. Investigating the Role of Tunable Nitrogen Vacancies in Graphitic Carbon Nitride Nanosheets for Efficient Visible-Light-Driven H2 Evolution and CO2 Reduction. ACS Sustain. Chem. Eng. 2017, 5, 7260-7268. [CrossRef]

243. Huang, Y.; Liu, J.; Zhao, C.; Jia, X.; Ma, M.; Qian, Y.; Yang, C.; Liu, K.; Tan, F.; Wang, Z.; et al. Facile Synthesis of Defect-Modified Thin-Layered and Porous g-C3N4with Synergetic Improvement for Photocatalytic H2Production. ACS Appl. Mater. Interfaces 2020. [CrossRef] [PubMed] 
244. Jiang, Y.; Sun, Z.; Tang, C.; Zhou, Y.; Zeng, L.; Huang, L. Enhancement of Photocatalytic Hydrogen Evolution Activity of Porous Oxygen Doped G-C3N4 with Nitrogen Defects Induced by Changing Electron Transition. Appl. Catal. B Environ. 2019, 240, 30-38. [CrossRef]

245. Ruan, D.; Kim, S.; Fujitsuka, M.; Majima, T. Defects Rich G-C3N4 with Mesoporous Structure for Efficient Photocatalytic H2 Production under Visible Light Irradiation. Appl. Catal. B Environ. 2018, 238, 638-646. [CrossRef]

246. Zhao, D.; Dong, C.; Wang, B.; Chen, C.; Huang, Y.; Diao, Z.; Li, S.; Guo, L.; Shen, S. Synergy of Dopants and Defects in Graphitic Carbon Nitride with Exceptionally Modulated Band Structures for Efficient Photocatalytic Oxygen Evolution. Adv. Mater. 2019, 31, 1903545. [CrossRef] [PubMed]

247. Shiraishi, Y.; Shiota, S.; Kofuji, Y.; Hashimoto, M.; Chishiro, K.; Hirakawa, H.; Tanaka, S.; Ichikawa, S.; Hirai, T. Nitrogen Fixation with Water on Carbon-Nitride-Based Metal-Free Photocatalysts with $0.1 \%$ Solar-to-Ammonia Energy Conversion Efficiency. ACS Appl. Energy Mater. 2018, 1, 4169-4177. [CrossRef]

248. Hao, D.; Liu, C.; Xu, X.; Kianinia, M.; Aharonovich, I.; Bai, X.; Liu, X.; Chen, Z.; Wei, W.; Jia, G.; et al. Surface Defect-Abundant One-Dimensional Graphitic Carbon Nitride Nanorods Boost Photocatalytic Nitrogen Fixation. New J. Chem. 2020, $44,20651$. [CrossRef] 